\title{
An investigation of private public partnerships using real options analysis
}

\author{
By
}

Zonghao. Chen

\begin{abstract}
A thesis
submitted to Victoria University of Wellington in fulfillment of the requirements for the degree of Master of Commerce in Finance
\end{abstract}

Victoria University of Wellington 


\section{Acknowledgement}

I am greatly indebted to my supervisor Prof Graeme Guthrie his consistent guidance and encouragement throughout the process.

I would also like to sincerely thank the examiners for their invaluable comments and suggestions.

Last but not least I would like to thank my parents for their support. 


\section{Table of Contents}

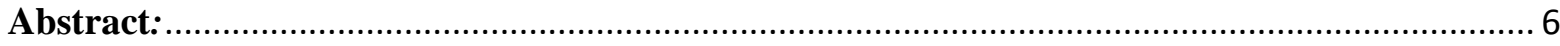

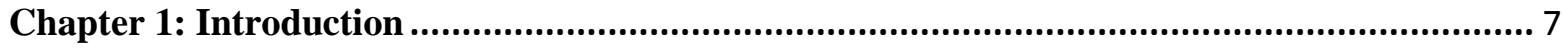

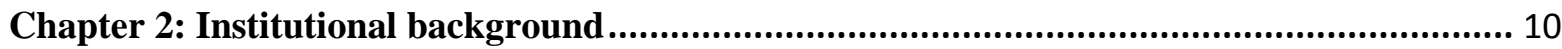

2.1 An introduction to the institutional structure in the UFB project .................................... 10

2.2 A brief discussion of private public partnership (PPPs) ................................................ 11

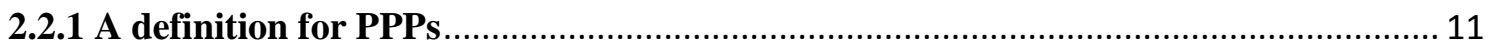

2.2.2 Advantages and disadvantages of PPPs over conventional procurement ................... 11

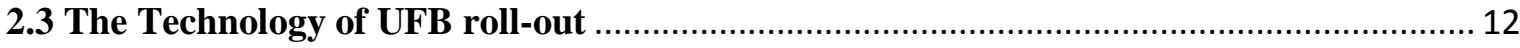

2.3.1 The difference between passing a premise and connecting a premise in UFB roll-out

2.3.2 The fibre deployment costs with different deployment techniques............................ 13

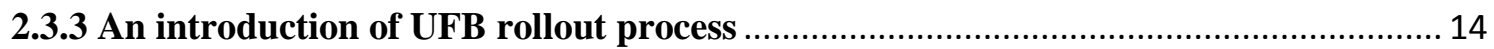

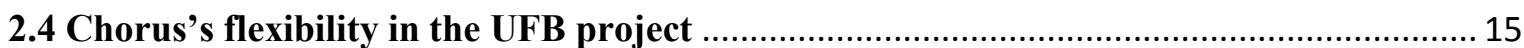

2.4.1 Chorus's flexibility in deciding the network deployment plan................................. 15

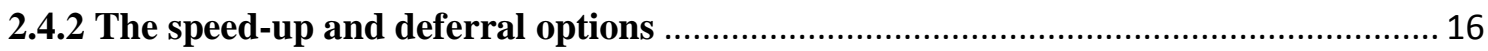

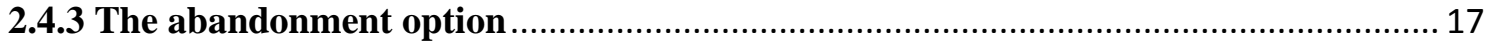

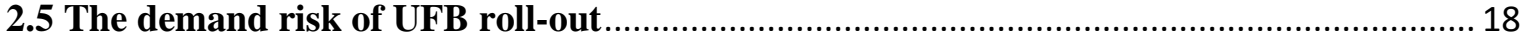

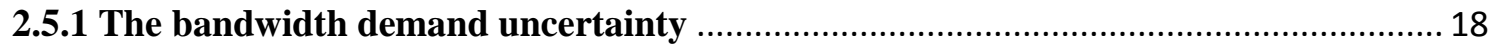

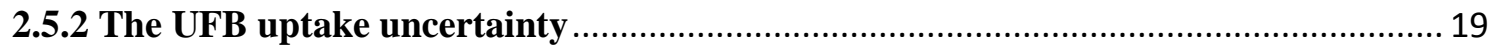

2.6 A brief introduction about the CFH financing in the Chorus's UFB roll-out ................... 19

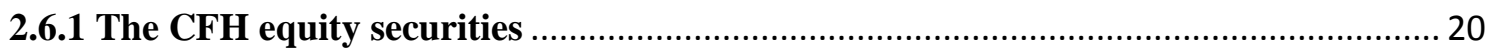

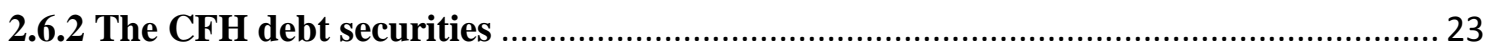

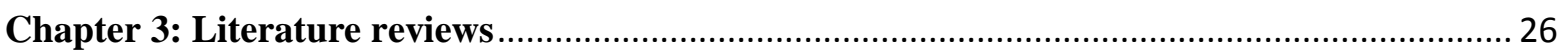

3.1 The literature review of PPPs in the scope of incomplete contracts ................................... 27

3.2 The literature review of PPPs in the scope of real options analysis.................................. 29

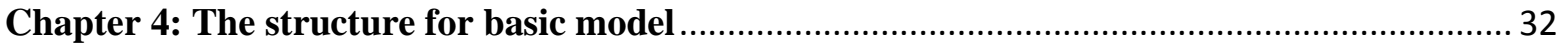

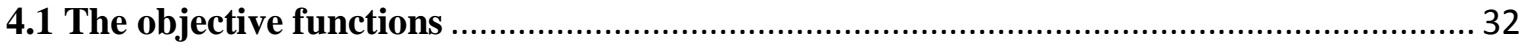

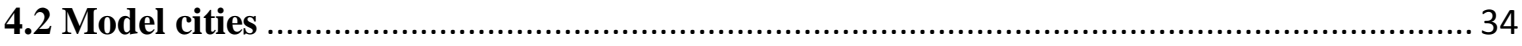

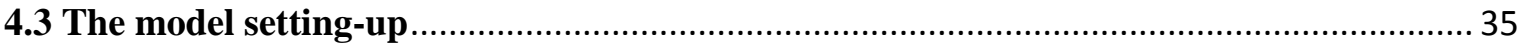

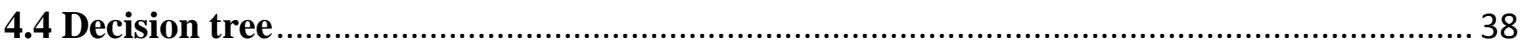

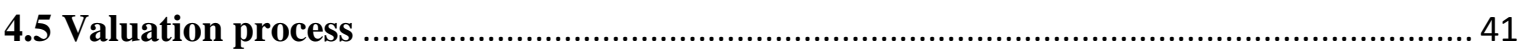

4.5.1 Valuation process for Chorus (Chorus optimal policy) ............................................ 41

4.5.2 Valuation process for CFH (CFH optimal policy) ........................................................ 45 
4.5.3 Valuation process for CFH (Chorus optimal policy) …............................................. 46

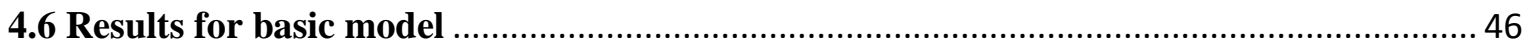

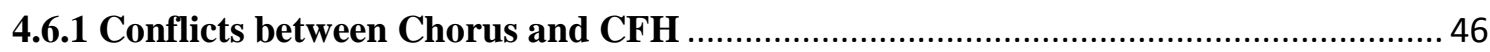

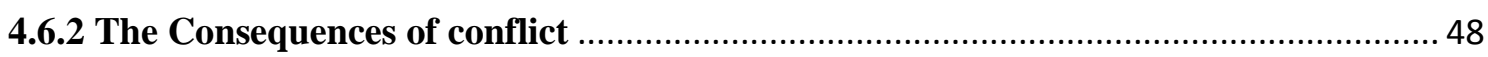

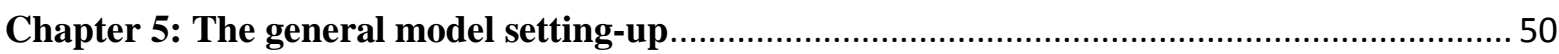

5.1 A shift from the basic model to the general model .............................................................. 50

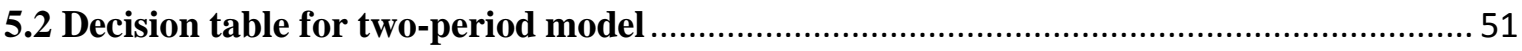

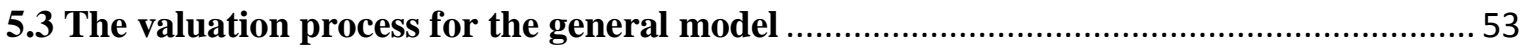

5.3.1 Valuation process of the general model for Chorus (Chorus optimal policy)............. 54

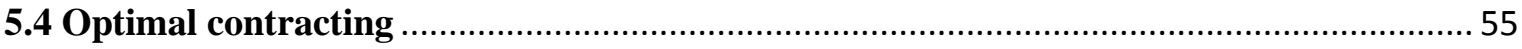

5.4.1 An add-on of subsidy schemes for the general model ............................................... 55

5.4.2 An add-on of the fine schemes for the general model ................................................. 58

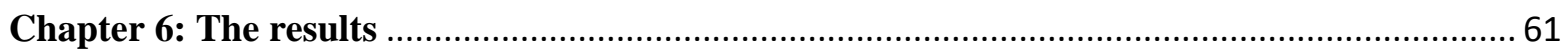

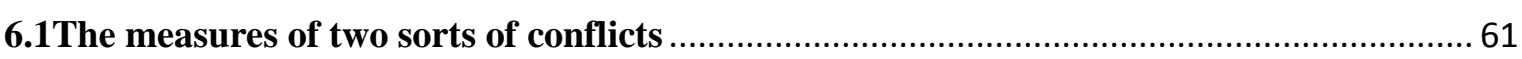

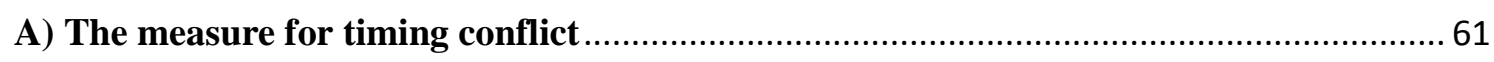

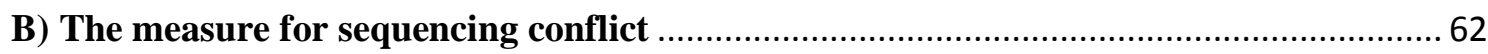

6.2 The conflicts between two parties in the baseline case ........................................................ 63

6.3 The sensitivity analysis on the conflicts in the baseline case...........................................67

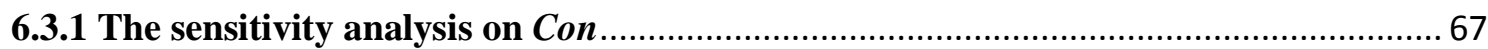

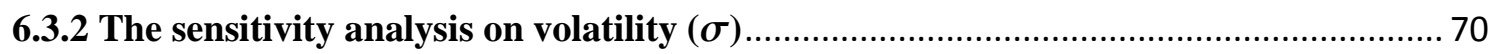

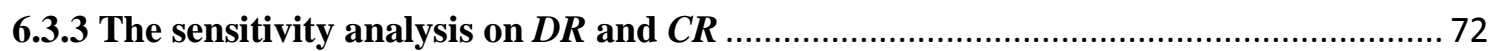

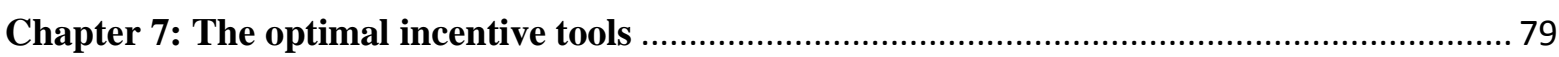

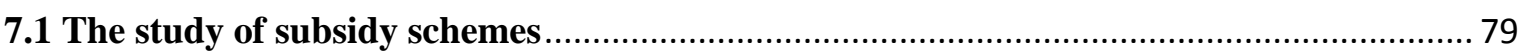

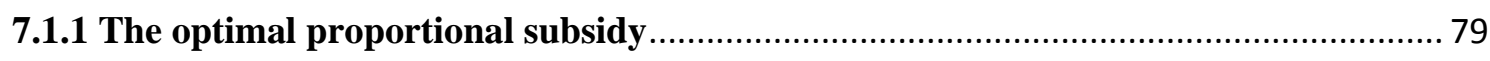

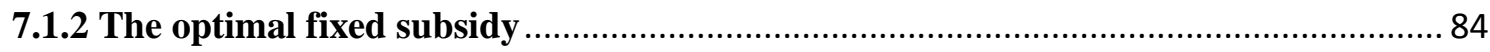

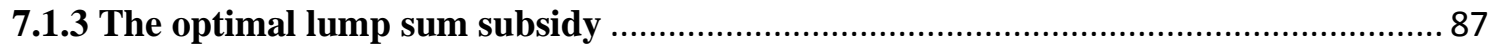

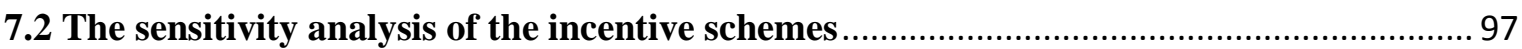

1) The sensitivity analysis of the proportional subsidy scheme .......................................... 97

2) The sensitivity analysis of the independent lump sum subsidy scheme ....................... 100

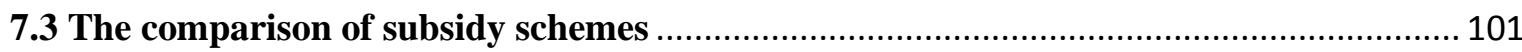

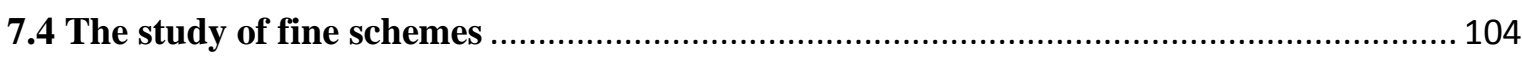

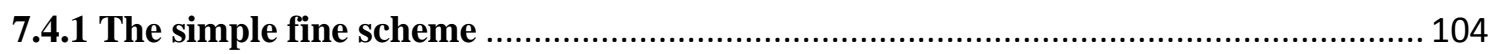

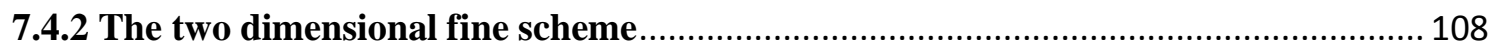

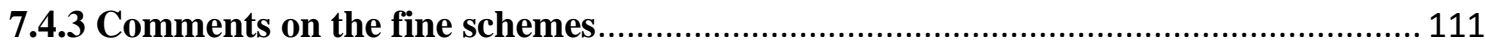


Chapter 8: Concluding remarks ...

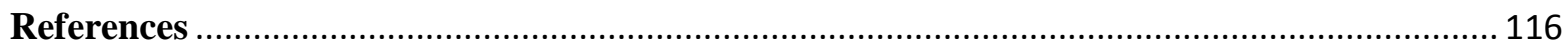




\begin{abstract}
:
This thesis develops a model that investigates aspects of New Zealand's largest publicprivate partnership project, the rollout of Ultra Fast Broadband. The model features four cities with different demand and construction-cost characteristics. It is used to study the different choices of the private party (Chorus) and the public party (Crown Fibre Holdings (CFH)). Using a real options approach, we identify two sorts of potential conflicts between the two parties: a timing conflict about the number of cities the two parties would like to develop in each period and a sequencing conflict about the order in which the UFB network is rolled out in different cities. Inspired by the incomplete contracting and information asymmetry literatures, we introduce several incentive schemes (including four subsidy schemes and two fine schemes) that help manage the possible conflicts. We compare both their ability to reduce the conflicts and their sensitivity to the model's underlying parameters. Overall, there are four main findings. First, the magnitude of the conflict is a non-monotonic function of the inter-city demand differences and the inter-city constructioncost differences; it is an increasing function of the ratio of consumer surplus to producer surplus and of demand volatility. Second, a demand-dependent lump sum subsidy has the best performance among all included incentive schemes in controlling the possible conflicts. Third, the conflict level becomes quite sensitive to the subsidy scheme in two cases. A) When either the inter-city demand differences or the inter-city construction-cost differences turn out to be modest; $B$ ) When either the ratio of consumer surplus to producer surplus or demand volatility turns out to be large. The above result may provide some suggestions in managing the optimal subsidy. Last but not least, the requirement that Chorus is willing to participate in the partnership means that the fine schemes are generally outperformed by the subsidy schemes. Relating our findings to the undertaking UFB project, we provide CFH with several practical suggestions that may improve its management of possible conflicts.
\end{abstract}




\section{Chapter 1: Introduction}

The Ultra-Fast Broadband project (UFB) was launched by the New Zealand government and outsourced to several infrastructure contractors to provide extremely fast Internet connections to $75 \%$ of the population in New Zealand by using optical fibre. The whole UFB project is estimated to cost $\$ 3.5$ billion and is expected to be finished at the end of 2019 . About $\$ 1.35$ billion of the investment in the UFB project is coming from the government and will be managed by the government entity Crown Fibre Holdings (CFH). The Local Fibre Companies (LFCs) and Chorus fund the rest of the expenditure on the UFB rollout.

The public sector often delivers various types of infrastructures to support economic growth. Since most infrastructure projects (like the UFB project) feature large-scale investments, a long duration of investment recovery and a complex risk profile, one typical problem that the public sector faces is budgetary shortfalls. Therefore, with the benefit of additional private financing, public private partnerships (PPPs) have developed into a widely used arrangement in delivering large-scale infrastructure projects.

This thesis develops as follows. Chapter 2 provides the institutional background for this thesis. First, we briefly mention the institutional structure in the UFB project and give a general picture about how the whole UFB project proceeds. We then discuss the main sources of demand uncertainty in the UFB project. Through a discussion about how CFH funds Chorus's UFB rollout, we demonstrate how CFH motivates Chorus to get a higher fibre demand through equity and debt subsidies.

Chapter 3 summarises two aspects of the literature in economics and finance that are relevant to this study. The literature in incomplete contracts argues about how managerial flexibility can be given to the private party, while the literature in real options analysis shows us how the managerial flexibility can be evaluated.

We introduce the basic version of our model in chapter 4. Like the more general model described in chapter 5, this one features four cities with different demand and constructioncost characteristics, which could be used to study the different choices of the private party (Chorus) and the public party (Crown Fibre Holdings). A decision tree has been constructed in order to analyse both parties' decision making in the basic model. We specify both parties' objective functions in the UFB project. Chorus cares only about the producer surplus (PS) and capital expenditures, whereas CFH cares about the total surplus (customer surplus (CS) 
plus the producer surplus (PS)) and capital expenditures. Since Chorus pays the same capital expenditure but receives fewer surpluses than $\mathrm{CFH}$, the company will be more "capital expenditure focussed" than CFH. With the objective functions (for both Chorus and CFH) and valuation processes, we identify two potential conflicts between Chorus and CFH. A) a timing conflict that Chorus wants to wait longer than $\mathrm{CFH}$ to invest; and $\mathrm{B}$ ) a sequencing conflict that Chorus wants to build up city LL (which is a low demand and low cost city) before city $\mathrm{HH}$ (which is a high demand and high cost city) while CFH wants to build up city HH before city LL. In the numerical example, we provide the two parties' policy tables to illustrate these two conflicts and, more importantly, demonstrate how to calculate the present value of the conflict.

However, the application of the basic model is restricted by its simple assumption that the construction required to roll out fibre to a single city can be completed in one period. In chapter 5, a shift from the basic model to the general model relaxes this restriction and allows the construction required to roll out fibre to a single city to be completed in more than one period. Similarly to the basic model case, a decision table has been provided to illustrate the possible actions that could be conducted by each player in the two-period model. In order to value the conflicts, we update the valuation processes for the general model. Moreover, two sorts of incentive schemes have been introduced to help reduce the possible conflicts between Chorus and CFH. The first family of incentive schemes we consider is made up of various forms of subsidy policies. This family includes a proportional subsidy scheme in which Chorus is paid proportional to its construction expenditures in each period, a fixed subsidy scheme that pays Chorus a fixed amount of money for each construction stage undertaken in each period, an independent lump sum subsidy scheme that pays Chorus a fixed amount of money for each city that has been finished in each period, and a demand relevant lump sum subsidy scheme in which as soon as a city has been finished, Chorus is paid an amount that depends on the demand character of the city. The second family of incentive schemes we consider imposes fines on Chorus if specific targets are not met. This family includes a simple fine scheme that requires Chorus to pay a fine to $\mathrm{CFH}$ corresponding to the number of cities not yet finished when the UFB contract expires, and a two-dimensional fine scheme in which Chorus is also required to pay a fine for each target it misses midway through the UFB contract. Both the description and evaluation formula have been provided for each incentive scheme. 
In order to study the timing conflict and the sequencing conflict separately, we introduce two kinds of conflict measures in chapter 6 . The timing conflict measure calculates the difference between $\mathrm{CFH}$ and Chorus in the total numbers of construction stages expected to be finished by certain fixed dates in the future. The sequencing conflict measure instead calculates the difference between conditional probabilities that $\mathrm{CFH}$ and Chorus each prefer to build up a city with high demand and high construction cost before a city with low demand and low construction cost. The results of numerical analysis in the baseline case (without any incentive scheme) demonstrate the conflicts and the corresponding social welfare loss. In the second half of chapter 6 , we conduct the sensitivity analysis to see how the present value of conflict is affected by the model's underlying parameters (that is, the ratio of demand in high-demand versus low-demand cities, the ratio of construction cost in high-cost versus low-cost cities, the relative magnitude of consumer surplus to producer surplus and demand volatility).

Chapter 7 studies the efficiencies of the incentive schemes in reducing the possible conflicts. For each subsidy scheme, we first demonstrate the relationship between the conflict level (the present value of conflict) and the subsidy scheme variable, thereby identifying the level of the subsidy that reduces the conflict to its minimum. We then explain the movement of the conflict level over each subsidy by using both the timing conflict measure and the sequencing conflict measure. After that, sensitivity analysis on the profile of the optimal subsidy has been conducted to analyse how the level of the optimal subsidy (correspondingly the level of minimum conflict) is affected by the model's underlying parameters (that is, the demand ratio, the cost ratio, the relative magnitude of consumer surplus to producer surplus, and demand volatility). In the comparison of subsidy schemes, the demand relevant lump sum subsidy scheme has been found to perform best in achieving the minimum conflict level. We conduct a similar study for the fine schemes and find that they are generally outperformed by the subsidy schemes due to their participation constraints.

We summarise some important findings of this thesis in chapter 8 . Relating to the undertaking UFB project, we provide CFH with several practical suggestions that may improve its management of possible conflicts. 


\section{Chapter 2: Institutional background}

\subsection{An introduction to the institutional structure in the UFB project}

Crown Fibre Holding (CFH), Chorus, Enable Network Limited (ENL), Waikato Networks Limited (WNL) and North Power Limited (NP) are the five parties involved in the national UFB network roll-out. Generally speaking, the national UFB network will be an outcome of the cooperation between the New Zealand government and the private sector. In particular, CFH has been established to manage the Government's investment in Ultra-Fast Broadband infrastructure and aims to achieve the government's UFB objectives. ${ }^{1}$ It can be treated as a representative of the government in the UFB project. For the four private sector entities (Chorus, ENL, WNL, NP), several different partnerships with CFH have been formed in the roll out of the UFB network. The following graph provides a brief summary of the UFB project's structure.

\section{Graph 2.1: The main players in the UFB project}

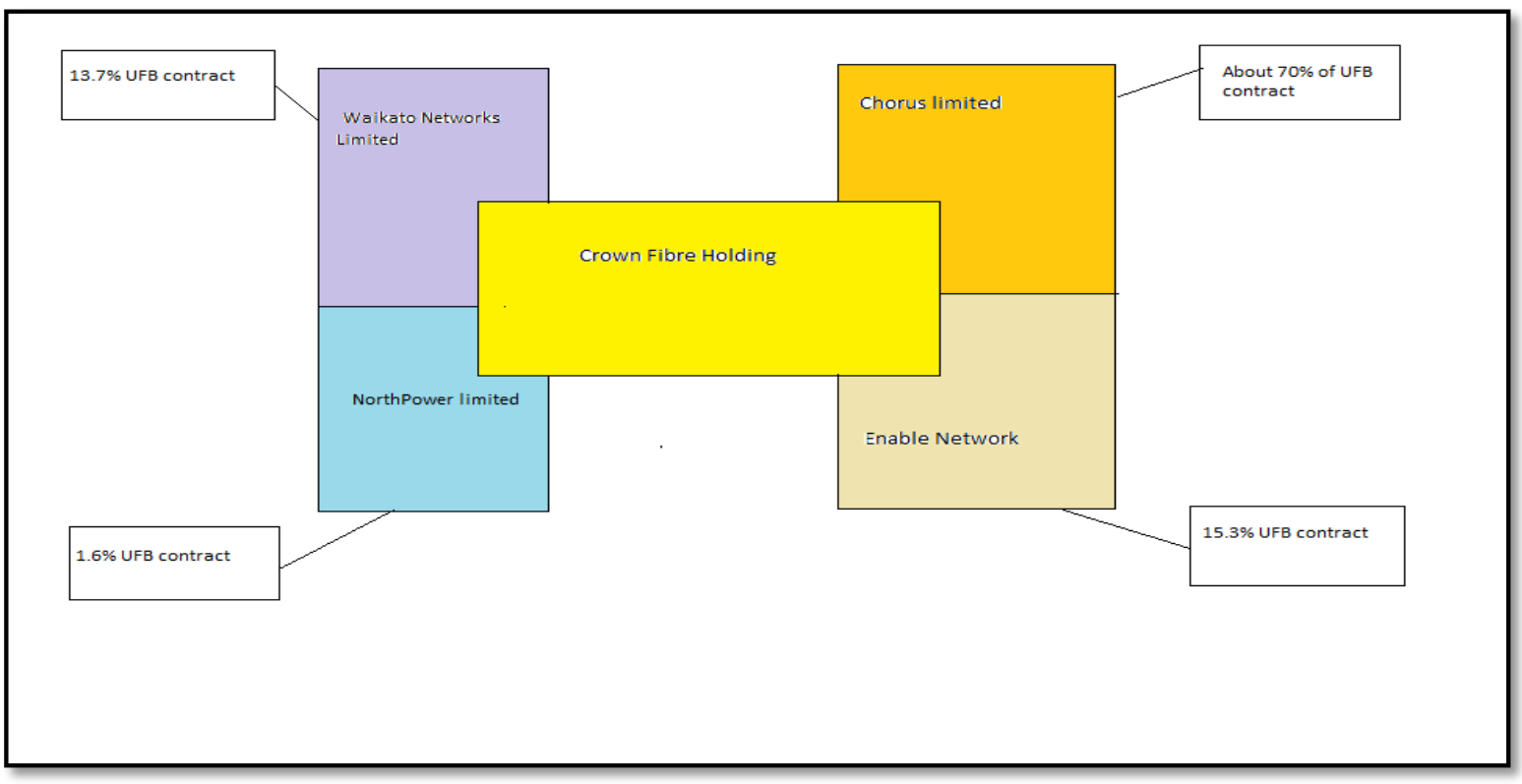

As we can see from graph 2.1, Crown Fibre Holdings partners with four individual contractors independently in order to deliver the UFB network to the whole country. Chorus

\footnotetext{
${ }^{1}$ The government's UFB objectives are to accelerate the roll-out of Ultra-Fast Broadband (UFB) to 75 per cent of New Zealanders over ten years, concentrating in the first six years on priority broadband users such as businesses, schools and health services, plus green field developments and certain tranches of residential areas.
} 
(which is separated from the Telecom group since Telecom won the tender) is the biggest player in the UFB project.

To simplify our research into the UFB infrastructure project, we ignore the participation of Waikato Networks Limited, Enable Networks and North Power, therefore giving us a simple structure of a private public partnership between $\mathrm{CFH}$ and Chorus.

\subsection{A brief discussion of private public partnerships}

\subsubsection{A definition of a private public partnership}

Although private-public partnerships (PPPs) are enjoying a rapid growth globally, a general agreement on the definition of PPPs has hardly been made in the academic literature. Some see it as a new governance tool, while others believe PPPs are just a "language game". Since the purpose of this thesis is not to end the PPP definition debate, we simply adopt a PPP definition which is consistent with the UFB project. That is,

The private public partnership is the cooperation of some kind of durability between public and private sectors in which they mutually develop products and services and share risks, costs and resources which are associated with these products. ${ }^{2}$

The UFB project fits this definition in three ways. First, PPPs cannot form by short term contracts (clearly, the UFB contracts between CFH and Chorus last more than twenty years). Second, risk sharing and cost and resource saving are two main purposes of PPP formation (CFH and Chorus are sharing some risks on the UFB rollout). Third, a product or a service had been mutually provided by public and private sectors as a consequence of their cooperation (which is the fibre service in UFB case).

\subsubsection{Advantages and disadvantages of PPPs over conventional procurement}

Compared with traditional procurement, PPPs have some advantages. First, PPPs are better in whole-of-life project evaluation. ${ }^{3}$ This opinion has been supported by several empirical studies. After evaluating 50 UK projects, MacDonald (2002) suggests that the capital expenditure of PPPs have only 1 per cent budget over-run on average, while traditional

\footnotetext{
${ }^{2}$ For more detail see Van Ham and Koppenjan (2001).

${ }^{3}$ The whole-of-life assessment under conventional procurement is carried out by a public agency which doesn't normally bear the financial consequences of getting it wrong. However, the whole-of-life assessment under PPPs is carried out by a private party which has arguably a stronger incentive than a government agency to be realistic about the prospects of a project. Therefore, the whole-of-life project evaluation under PPPs is normally better than that in traditional procurement.
} 
procurement projects have 47 per cent cost over-run on budget on average. HM Treasury (2003) studied 61 PPP projects and found only 11 per cent of them were behind schedule, and none of them were over the public sector budgets. The UK National Audit Office (NAO, 2003; 2005) compared the construction performance of PPPs and traditional procurement. Only 30 per cent of conventional projects were on time and only 27 per cent were within budget. By contrast, the PPPs were largely delivered on time or early (76 per cent versus 30 per cent) and on budget (78 per cent versus 27 per cent). Besides, some believe that PPPs normally come up with better design and operation and less whole-of-life $\operatorname{costs}^{4}$ (such as Katz (2006), Grimsey and Lewis (2007)).

On the other hand, the application of PPPs is not a free lunch. That is, PPPs are typically much more complicated and higher tendering, contracting and renegotiation costs often occur (Katz (2006), Grimsey and Lewis (2007)).

\subsection{The Technology of UFB roll-out}

\subsubsection{The difference between passing a premise and connecting a premise in UFB roll-} out

The amount of subsidy received by Chorus depends on the number of premises passed by the UFB network, as well as the number of premises connected to the network. To avoid ambiguity, we give the definitions of passing a premise and connecting a premise (either from $\mathrm{NIPA}^{5}$ or $\mathrm{CFH}$ annual report).

\section{Passed means:}

Where premises have been passed with fibre optic cable, or conduit capable of having fibre optic cable inserted, and the premises are able to be connected to the nearest point of the premises' boundary.

\section{Connection means:}

(a) The cable joining the Fibre Access Point to the External Termination Point of a Premises, with such cable to be either from the pit on the adjoining boundary of two properties where

\footnotetext{
${ }^{4}$ Compared with conventional procurement, the designers and builders under PPPs normally have a financial stake in the project over its whole life. Therefore, the designers and builders are more likely to have a strong incentive to design features and construction standards so they are optimized against the long-term cost of maintenance and operational requirements.

${ }^{5}$ NIPA is the abbreviation of Network Infrastructure Project Agreement.
} 
the Fibre Access Point is located in underground deployment, or from the pole nearby to a number of Premises in aerial deployment; and

(b) All other infrastructure (excluding Communal Infrastructure) necessary to enable the provision of the Specified Layer 1 Services and Specified Layer 2 Services to the relevant End User, as required under Schedule 5 (Service Levels);

The difference between passing a premise and connecting a premise is that passing provides the end users capability of connecting their premises to the fibre network, while connecting means the end users are going to sign up the fibre services and Chorus will send people to connect end users' premises to the fibre access point.

\subsubsection{The fibre deployment costs with different deployment techniques}

On the UFB rollout, Chorus may deploy fibre optic cables in the access network with a variety of techniques. The costs of deployment depend on the deployment method that Chorus applies. Table 2.1 provides a comparison of fibre deployment costs with the most common deployment techniques.

\section{Table 2.1: A comparison of fibre deployment costs with different deployment techniques}

\begin{tabular}{|c|c|c|}
\hline Deployment techniques & The costs associated & Comments \\
\hline Aerial deployment & $\begin{array}{l}\text { The typical aerial cost is } \\
\text { around } \$ 30-\$ 50 \text { per meter. }\end{array}$ & $\begin{array}{l}\text { Aerial deployment is a low cost approach for the } \\
\text { deployment of fibre optic cable where existing } \\
\text { poles. However, this method is not available in any } \\
\text { urban areas which already have all underground } \\
\text { facilities. Besides, some Local Councils vigorously } \\
\text { discourage the deployment of new aerial plant (for } \\
\text { visual pollution), which restricts the application of } \\
\text { aerial deployment in New Zealand. }{ }^{6}\end{array}$ \\
\hline Shallow trenching & $\begin{array}{l}\text { The typical cost for shallow } \\
\text { trenching is around } \$ 70-90 \\
\text { per meter. }\end{array}$ & $\begin{array}{l}\text { Shallow trenching has not been used in New } \\
\text { Zealand due to perceived issues with the negative } \\
\text { impact of this deployment on road maintenance. } \\
\text { However, there are modern approach to implement } \\
\text { this technique in a way can minimize any impact on } \\
\text { road maintenance. }\end{array}$ \\
\hline
\end{tabular}

\footnotetext{
${ }^{6}$ Milner (2009) claims that about $30 \%-40 \%$ of homes and businesses on the UFB rollout might be suitable for aerial deployment under ideal conditions.
} 


\begin{tabular}{|c|c|c|}
\hline Micro trenching & $\begin{array}{l}\text { The cost of micro trenching } \\
\text { is around } \$ 50-70 \text { per meter } \\
\text { along street curbs or } \$ 30-50 \\
\text { per meter alongside grass } \\
\text { verges. }\end{array}$ & $\begin{array}{l}\text { This may be the most promising approach to } \\
\text { achieve reduced costs for fibre deployment in New } \\
\text { Zealand. However, the micro trenching is still under } \\
\text { the trial to see how it works in New Zealand road } \\
\text { conditions. }\end{array}$ \\
\hline Mole plough trenching & $\begin{array}{l}\text { The typical cost for } \\
\text { deployment of fibre using a } \\
\text { mole plough lies in the } \\
\text { range of } \$ 20-40 \text { per meter. }\end{array}$ & $\begin{array}{l}\text { Although this method comes with low deployment } \\
\text { costs, it can only be operated in soft ground. } \\
\text { Therefore, the amount of mole plough trenching } \\
\text { that could be applied in fibre urban deployment } \\
\text { would be very small. }\end{array}$ \\
\hline Directional drilling & $\begin{array}{l}\text { The typical cost for } \\
\text { directional drilling is around } \\
\$ 50-70 \text { per meter. }\end{array}$ & $\begin{array}{l}\text { The cost for directional drilling is highly variable } \\
\text { with ground conditions. It is widely applied in cities } \\
\text { like Christchurch and Hamilton, but can be quite } \\
\text { challenging in cities like Wellington, Dunedin and } \\
\text { parts of Auckland. Besides, directional drilling } \\
\text { normally requires to be combined with traditional } \\
\text { open trenching. }\end{array}$ \\
\hline Open trenching & $\begin{array}{l}\text { Simple trenching can cost as } \\
\text { little as } \$ 120-150 \text { per meter, } \\
\text { while the costs can explode } \\
\text { to } \$ 500-600 \text { per meter when } \\
\text { substantial concrete cutting, } \\
\text { rock cutting and } \\
\text { reinstatement is required. }\end{array}$ & $\begin{array}{l}\text { Open trenching is a highly labour intensive process } \\
\text { and so the costs are high. Due to the costs, open } \\
\text { trenching is treated as a last resort in any urban } \\
\text { fibre deployment. }\end{array}$ \\
\hline
\end{tabular}

* sourced from Fibre-to-the-Premise Cost Study (produced by Milner Consulting Limited at February 2009)

Within the defined UFB coverage area, there are widespread variations in deployment conditions. Since the different deployment conditions may require a different combination of deployment methods, it is highly likely that the deployment costs vary widely over the UFB coverage areas. Even if deployment conditions are homogeneous over the country, the total deployment costs are still heterogeneous across the UFB coverage area due to the variation in population density.

\subsubsection{An introduction to the UFB rollout process}

\footnotetext{
${ }^{7}$ Some roading engineers claim, with the micro trenching, there is a high risk of roads falling apart and of fibre being damaged by stress from traffic or cut by contractors when they are digging to repair other utilities. This is sourced from http://www.stuff.co.nz/business/3832735/UFB-trenching-trials-to-begin-in-September.
} 


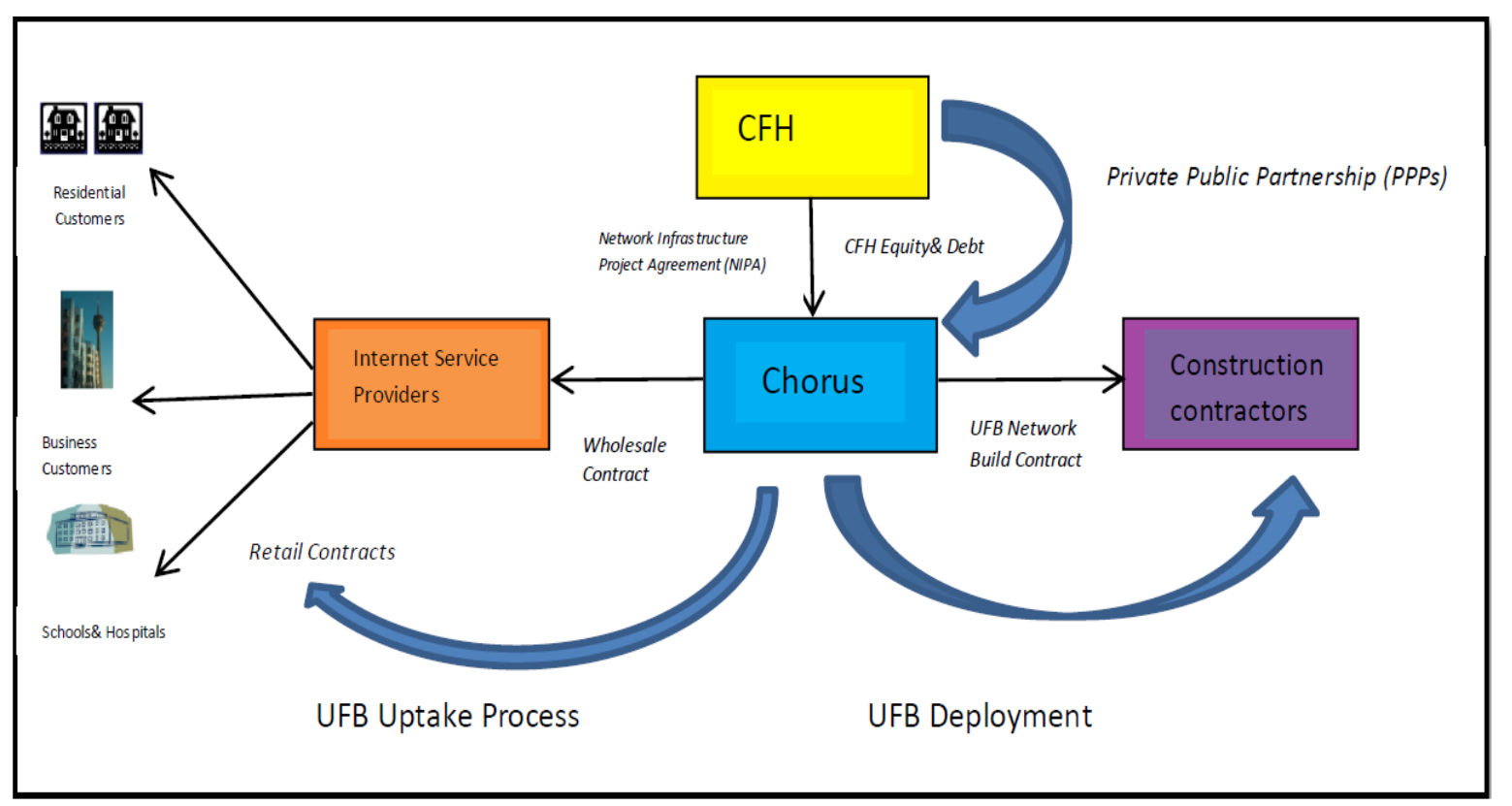

As we can see from graph 2.2, the UFB rollout has two main processes. The first one is the fibre network deployment (in this process, premises will be passed with fibre optic cable), while the second one is the fibre uptake (in this process, the end customers will be connected to the fibre network and start to enjoy the UFB services). In the fibre network deployment process, Chorus delegates the network infrastructure construction to several construction contractors through the build contracts. In the fibre uptake process, internet service providers (ISPs) act as an intermediary between Chorus and end users. Actually, Internet service providers set up and advertise their own fibre schemes in order to get the end users. ${ }^{8}$ Then, they place the orders to Chorus and ask Chorus to connect the end customers to the fibre network. ${ }^{9}$ In the supply side, internet service providers help Chorus communicate and pass fibre services to the end users. Of course, the money from end users will go to the service provider first and Chorus second.

\subsection{Chorus's flexibility in the UFB project}

\subsubsection{Chorus's flexibility in deciding the network deployment plan}

A network deployment plan is an operative document which set out the plan for Chorus to deploy the fibre network during a certain timeframe. In general, a network deployment plan summarizes Chorus's timing and ordering in the fibre network construction during a certain

\footnotetext{
${ }^{8}$ The retail contracts link the end users to internet service providers.

${ }^{9}$ The wholesale contracts link the internet service providers to Chorus.
} 
timeframe. Therefore, Chorus's decision-making power to change a deployment plan influences its level of managerial flexibility in the UFB network deployment. For example, at each review period of the master network deployment plan $^{10}, \mathrm{CFH}$ will provide Chorus with CFH's prioritization criteria ${ }^{11}$ and the minimum number of premises to be passed in each network deployment plan period for the period leading up to the next review period. On the other hand, Chorus will provide $\mathrm{CFH}$ with:

a) The Company's Patches which make up the Coverage Area;

b) Candidate Areas within each Patch;

c) Start and completion build milestones for each Candidate Area;

d) Expected annual Premises Passed (including Priority Users) in each Patch and Candidate Area; and

e) Build periods per Candidate Area (if any).

Although there are some constraints ${ }^{12}$, the fact that Chorus provides part of the information to review the deployment plan indicates that some decision-making power in the deployment review has been passed to Chorus. Therefore, Chorus has some level of managerial flexibility in the UFB network deployment.

\subsubsection{The speed-up and deferral options}

In the UFB project, Chorus is allowed to speed up the fibre network deployment process. ${ }^{13}$ Besides, NIPA may grant Chorus some flexibility to defer the network deployment in certain coverage areas: ${ }^{14}$

The company will be entitled to a reasonable extension to the relevant Milestone Date (and, to the extent reasonably necessary, any subsequent Milestone Dates) for some Delay Causes, provided that such extension is proportionate to the duration of the delay, taking into

\footnotetext{
${ }^{10}$ The master network deployment plan will be used to guide each subsequent Deployment Plan (the network deployment plan specifies the procedure of fibre network deployment in each year).

${ }^{11}$ This means any information or guidance to be provided to the Company by CFH indicating the preferred order of Area Units and/or Candidate Areas.

${ }^{12}$ For example, Chorus needs to follows CFH's prioritization criteria and the minimum number of premises to be passed in each deployment period. Besides, CFH has a final approval right to change the network deployment plan.

${ }^{13}$ In 2012 CFH annual financial report, the chairman of CFH states that the Government's goal of substantially passing more than 70,000 premises by 30 June was comfortably exceeded (76,000 premises substantially passed).

${ }^{14}$ For more detail on all plan details in this section, see Network Infrastructure Project Agreement.
} 
account all measures the Company can reasonably take to mitigate the effect of the delay and make up lost time;

One of the Delay Causes is:

The number of Premises Passed for the Performance Milestone being more than 5\% higher than the estimated number of Premises to be Passed for the Performance Milestone (as set out in the Network Deployment Plan),

The above terms mean that if Chorus is able to speed up the fibre rollout in some coverage areas (such as the areas with low capital expenditure) to make the total number of premises passed be 5\% higher than the target in the deployment plan, Chorus will be entitled to exercise a deferral option to rollout fibre in the other coverage areas (such as the areas with high capital expenditures). In other words, Chorus has some flexibility to defer the deployment process in certain areas when the certain circumstance has been met.

\subsubsection{The abandonment option}

According to NIPA, Chorus has the option to pay CFH a penalty and terminate its agreement with CFH for designing and building up the UFB network, with the penalty capped at $\$ 360$ million. ${ }^{15}$ In practice, this arrangement grants Chorus an abandonment option, which allows Chorus to sell the UFB project back to $\mathrm{CFH}$ for a fixed strike price $\mathrm{K}$ of $-\$ 360$ million. The abandonment option is thus similar to a standard put option.

For a standard put option, reducing the strike price reduces the margin between the strike price and the value of the underlying asset, therefore decreasing the present value of the put option. Similarly, for this abandonment option, increasing of the penalty cap raises the cash outflow that Chorus needs to incur to abandon the project. Therefore, it reduces the present value of the abandonment option.

As we can see from graph 2.3, the value of the abandonment option becomes positive as long as the present value of the UFB project for Chorus is less than the strike price $\mathrm{K}$ ( $\mathrm{K}=-\$ 360$ million). When the value of $\mathrm{K}$ decreases (when the penalty cap increases), the present value line of the abandonment option moves to the left, which gives Chorus less

\footnotetext{
${ }^{15}$ This calculation is based on $\$ 350$ million (Maximum Aggregate Liability) plus $\$ 50,000$ a day for up to six months (Material Breach Liquidated Damages). For more detail see Network Infrastructure Project Agreements (NIPA) or http://www.stuff.co.nz/business/industries/9457779/Broker-suggests-Chorus-shouldwalk-away.
} 
protection from the downside risks. Besides, the value of the abandonment option may vary over the uncertainties of capital expenditures and fibre demand in the UFB project (since capital expenditures and fibre demand determine the present value of UFB project for Chorus).

\section{Graph 2.3: the present value of the abandonment option}

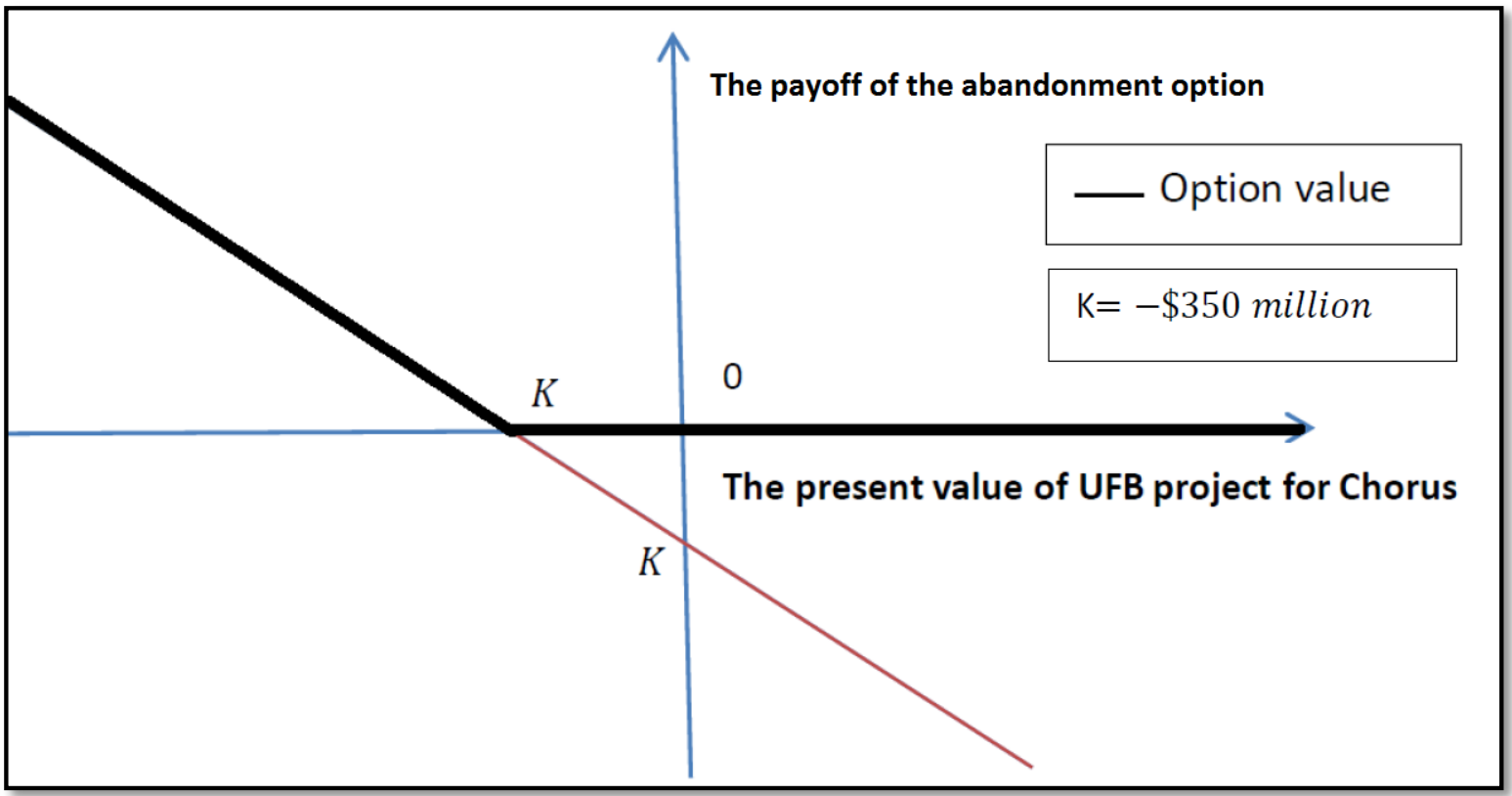

\subsection{The demand risk of the UFB roll-out}

In a regulated broadband wholesale market like New Zealand, the revenue streams of the network operators (Chorus) are determined by two main uncertainties. The first one is the average bandwidth demand uncertainty and the second one is the UFB connection number uncertainty.

\subsubsection{The bandwidth demand uncertainty}

The average bandwidth demand here describes the speed that the average customer requires for their UFB plans. As we know that the total revenue of Chorus in any certain time period simply equals the product of existing connection number and average charged price, the total revenue of Chorus from the UFB Project changes if either term changes. 
Since the fibre price caps have been agreed between CFH and Chorus in the UFB contract, the unit prices Chorus can charge its customers could be treated as fixed over time. ${ }^{16}$ The only source of volatility to the average charged price may come from people's decisions among different speed plans. For example, the average charged price may go up if more customers want to choose a "fast" UFB plan rather than a "slow" UFB plan and vice versa. And what does generally change people's migration decisions to different speed UFB plans over time? The popularity of internet TV, Cloud services, HD videos or any other coming internet high-tech services may have impact on the numbers of customers choosing to migrate from a copper plan to a "fast" UFB plan or a "slow" UFB plan. For example, if the cloud services become much more successful, more people may want to choose a "fast" UFB plan since a good experience of cloud service requires a fast upload speed.

\subsubsection{The UFB uptake uncertainty}

The second main source of total revenue volatility is contributed by the number of households connecting to the UFB network (take-up). What contributes to the volatility of the UFB connection number? As we discussed above, the popularity of "new" internet technologies may influence the demand of bandwidth, which may partially determine the number of existing copper network customers wanting to migrate to the fibre network and the number of potential new internet users who would like to connect to the fibre network. Besides, the relative prices between copper and fibre may dramatically change customers' incentives of migration from copper to fibre. ${ }^{17}$ Since New Zealand's broadband wholesale market is currently under regulation, the change of relative prices between copper and fibre could be grouped as political risk.

\subsection{A brief introduction about the CFH financing of Chorus's UFB roll-out}

On the UFB rollout, Chorus will self-fund the design and build work and carry the risk of any cost overruns in the network build. CFH, as a public sector support in the UFB project, will invest up to $\$ 929$ million in Chorus progressively during the fibre deployment as

\footnotetext{
${ }^{16}$ Although the fibre contract prices are changing over the UFB period, these changes are predetermined by $\mathrm{CFH}$ and Chorus in the UFB contracts. Therefore, there is not any uncertainty involved in fibre contract prices. For more detail see "Submission in response to the Commerce Commission's Draft Determination to amend the price payable for the regulated service Chorus' unbundled bitstream access made under s 30R of the Telecommunications Act 2001".

${ }^{17}$ If the reduction of copper service wholesale prices unlikely pass through to the end users, the margin of retail service providers in copper service will be enhanced and therefore encourage retail service providers to stay in copper service rather than jumping to fibre.
} 
premises are passed and network build stages are completed, including UAT. ${ }^{18} \mathrm{CFH}$ 's investment in Chorus is essentially a 50:50 mix of debt and equity. To allow $\mathrm{CFH}$ to participate in the upside of the financial performance of the company, Chorus will also issue one equity warrant to $\mathrm{CFH}$ for each $\$ 1$ of $\mathrm{CFH}$ equity securities. However, the present value of CFH warrants is quite small when compared to the CFH equity and debt. ${ }^{19}$ Therefore, we focus mainly on the CFH equity and debt.

Although CFH's initial investment in Chorus's UFB rollout is independent of both demand and capital expenditure, Chorus has a relatively large liability to pay back CFH's initial investment in the future if the fibre uptake (the demand of fibre) turns out to be below a certain level. Actually, in present value terms, Chorus receives more subsidy from CFH if the demand of fibre becomes relatively high, which works as an incentive scheme from $\mathrm{CFH}$ that motivates Chorus to reach a higher demand on the UFB rollout. The rest of the chapter demonstrates how this incentive scheme works using CFH equity and debt securities.

\subsubsection{The CFH equity securities}

\section{The description of CFH equity securities}

According to the agreement between Chorus and $\mathrm{CFH}$, half of the $\mathrm{CFH}$ investment in Chorus will be exchanged for Chorus equity securities, which are known as CFH equity securities. However, these equity securities bear no dividend payment until 2025 and partially attract dividends payment after 2025.The portion of CFH equity securities that attract dividends in each year depends on the fibre uptake rate in 2019 (whether the fibre uptake rate goes over 20\%). The CFH equity securities could be treated as a kind of preferred stock since they generally carry no right to vote at Chorus shareholder meetings, but offer a repayment preference on liquidation. Moreover, compared to the normal preferred stocks, the CFH equity securities are redeemable and dividend non-payable in a certain period (between 2012 and 2025).

\section{The cash flow of CFH equity securities}

Table 2.2 shows the dividend scheme for $\mathrm{CFH}$ equity securities from 2025 to 2036 . The dividend payment is dependent on which scenario Chorus is going to sit in the 30 June 2020

\footnotetext{
${ }^{18}$ UAT refers the user acceptance testing.

${ }^{19}$ In 2012 CFH annual report, the fair value of CFH warrant is only 11,000 NZ dollars, while the fair value of $\mathrm{CFH}$ securities are more than 10 million NZ dollars. Actually, it is unlikely that these warrants will be exercised by $\mathrm{CFH}$ unless Chorus's stock return exceeds a return hurdle of $16 \%$ per annum in the relevant period.
} 
uptake test. For example, if the fibre uptake is less than or equal to $20 \%$ in $2020, \$ 155$ million (33.3\%) of CFH equity is required to pay a dividend in 2025. In contrast, if the fibre uptake is more than $20 \%$, only $\$ 86$ million (18.5\%) of $\mathrm{CFH}$ equity is required to pay a dividend in 2025. Besides, the portion of $\mathrm{CFH}$ equity securities that attracts a dividend is increasing and cumulating over time. Not later than 2036, all CFH equity securities attract dividends.

Table 2.2: CFH equity securities' redemption and dividend table (\$m)

\begin{tabular}{|c|c|c|c|c|c|c|}
\hline \multirow[t]{2}{*}{30 June 2020 test } & 30 June (\$ million in dollar term) & \multicolumn{5}{|c|}{30 June ( in percentage term) } \\
\hline & $\begin{array}{lllll}2025 & 2030 & 2033 & 2035 & 2036\end{array}$ & 2025 & 2030 & 2033 & 2035 & 2036 \\
\hline Senario A & \multicolumn{6}{|c|}{ Fibre uptake less than or equal to $20 \%$} \\
\hline $\begin{array}{l}\text { Equity on which dividends } \\
\text { became payable }\end{array}$ & $155 \quad 310$ & $33.3 \%$ & $66.7 \%$ & & $100 \%$ & \\
\hline Senario B & \multicolumn{6}{|l|}{ Fibre uptake greater than $20 \%$} \\
\hline $\begin{array}{l}\text { Equity on which dividends } \\
\text { became payable }\end{array}$ & $172 \quad 300$ & $18.5 \%$ & $36.9 \%$ & $64.6 \%$ & & $100 \%$ \\
\hline
\end{tabular}

\section{Graph 2.4: A comparison of dividend rate distribution between fibre uptake states}

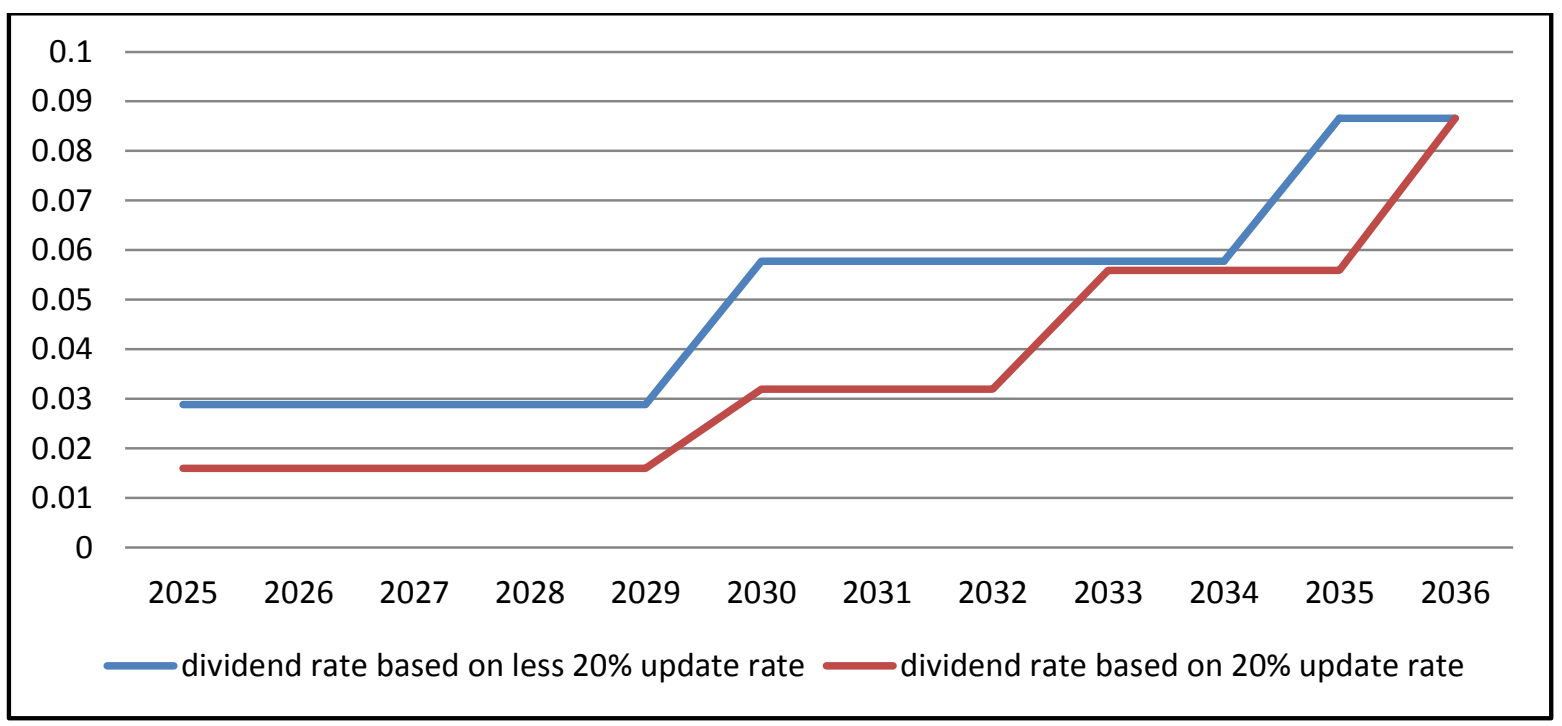

Graph 2.4 shows a comparison of dividend rate distribution between two fibre uptake states (from 2025 to 2036). The horizontal axis represents the year and the vertical axis represents the cumulative dividend yield of CFH equity securities for that particular year. As we can see clearly from graph 2.4 , the blue line dominates the red line in all periods. This indicates that the $\mathrm{CFH}$ equity securities are less valuable in the state $\mathrm{H}$ (when the uptake rate is over 
$20 \%$ ) than in the state $\mathrm{L}$ (when the uptake rate is equal or less than 20\%). In other words, CFH motivates Chorus to work harder in the UFB infrastructure by offering it an equity subsidy with a higher present value.

\section{The present value calculation of CFH equity subsidy}

In order to get the present value of the CFH equity subsidy, we need to estimate the present value of CFH's equity investment first. According to CFH's financial statement in 2012, for each premises passed, $\mathrm{CFH}$ needs to subscribe to $\$ 559$ of equity securities and $\$ 559$ of debt securities from Chorus, so the cash flows of CFH's equity investment depend on the UFB infrastructure process. In this section, the present value of CFH's initial investment is calculated by discounting the scheduled equity investment by the risk-free discount rate. ${ }^{20}$

\section{Graph 2.5: The numbers of premises expected to have been built by Chorus in each}

\section{financial year}

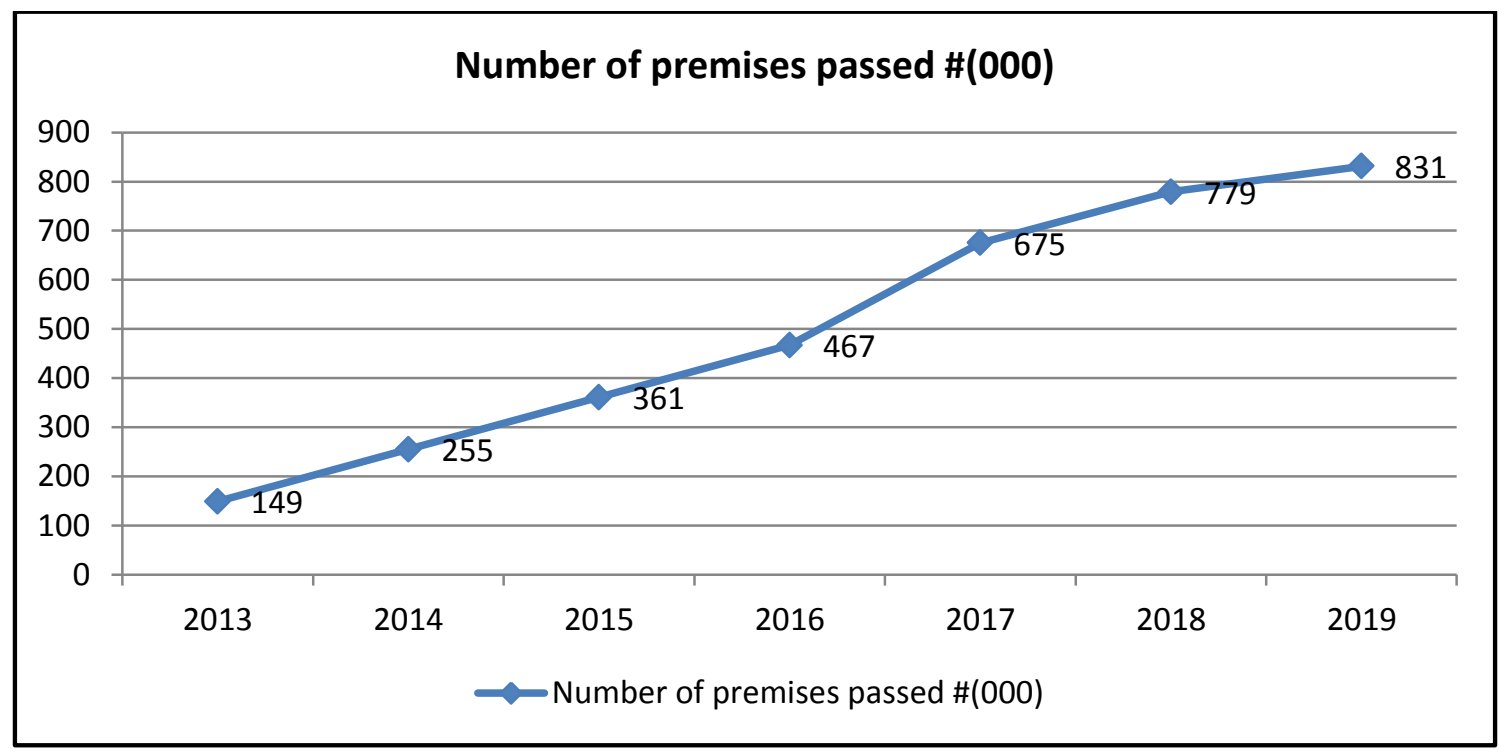

*sourced from Crown Fiber Holding 2012 annual report

Graph 2.5 provides information about the numbers of premises that are going to be passed by Chorus in the next seven financial years, which indicates the expected future cash flows of CFH's equity investment in Chorus's UFB roll-out. The expected cash flow of CFH's equity investment could be calculated by multiplying the numbers of premises passed by Chorus in each financial year by $\$ 559$. The present value of $\mathrm{CFH}$ initial equity investment in Chorus's UFB roll-out equals $\$ 429$ million.

\footnotetext{
${ }^{20}$ Since CFH is a New Zealand government entity, CFH's initial investment in Chorus's UFB roll-out is guaranteed by the New Zealand government. Therefore, we may treat CFH's initial investment as a series of risk-free cash flows.
} 
Next step, we are going to calculate the present value of CFH's equity securities. From above, the CFH equity securities are a kind of redeemable preferred share, which is nondividend bearing in the first 12 years. We assume the preferred stocks will not be redeemed before they attract full dividends to simplify our evaluation. As we know the required dividend rate for the equity is $8.68 \%$ (2.68\% 180 bank rate $+6 \%$ margin rate). We can treat 1 dollar of CFH preferred stock in its full face value when they start to attract full dividends (2035 in state L, 2036 in state H). Then, we discount the 1 dollar from 2035 (2036 in state H) back to 2013 to get the present value of the principal. Respectively, the present value of the equity principal in two uptake states from our calculation is \$74.5 million and \$68.6 million. Similarly, the present value of the dividend payment for each state equals $\$ 61.26$ million in state $\mathrm{L}$ and $\$ 41.24$ million in state $\mathrm{H}$. We combine the present value of principal with the present value of dividend payments to get the present value or equity securities in each state.

In 2013, the present values of CFH equity securities are $\$ 135.8$ million for state $\mathrm{L}$ and $\$ 109.8$ million for state $\mathrm{H}$. Accordingly, the present value of equity subsidies equals $\$ 293.2$ million at state $\mathrm{L}$ and $\$ 319.2$ million at state $\mathrm{H}$.

\subsubsection{The CFH debt securities}

\section{The description of CFH debt securities}

The CFH debt securities are unsecured and non-interest bearing. They carry no voting rights and will be required to be repaid fully at certain dates, with the repayment dates determined by the result of the fibre uptake test at 30 June 2020. If the NIPA is terminated under certain termination events, $\mathrm{CFH}$ can elect whether to continue to hold the $\mathrm{CFH}$ debt securities or require Chorus to repay these in cash.

\section{The cash flow of CFH debt securities}

Table 2.3 shows the repayment scheme for CFH debt securities from 2025 to 2036 . The debt payment is dependent on which scenario Chorus is going to sit in the 30 June 2020 uptake test. That is, $\$ 155$ million of debt Chorus needs to repay CFH in 2025 when the fibre uptake is low, while $\$ 86$ million of debt Chorus needs to repay CFH in 2025 when the fibre uptake is high. 
Table 2.3: CFH's debt securities repayment profile (\$m)

\begin{tabular}{|c|c|c|c|c|c|c|}
\hline \multirow[t]{2}{*}{30 June 2020 test } & 30 June (\$ million in dollar term) & \multicolumn{5}{|c|}{30 June ( in percentage term) } \\
\hline & $\begin{array}{lllll}2025 & 2030 & 2033 & 2035 & 2036\end{array}$ & 2025 & 2030 & 2033 & 2035 & 2036 \\
\hline Senario A & \multicolumn{6}{|c|}{ Fibre uptake less than or equal to $20 \%$} \\
\hline Debt repayment & 155 & $33.3 \%$ & $33.3 \%$ & & $33.3 \%$ & \\
\hline Senario B & \multicolumn{6}{|l|}{ Fibre uptake greater than $20 \%$} \\
\hline Debt repayment & 129 & $18.5 \%$ & 18.5 & $27.7 \%$ & & $35.4 \%$ \\
\hline
\end{tabular}

Graph 2.6: A comparison of CFH debt repayment schemes between fibre uptake states

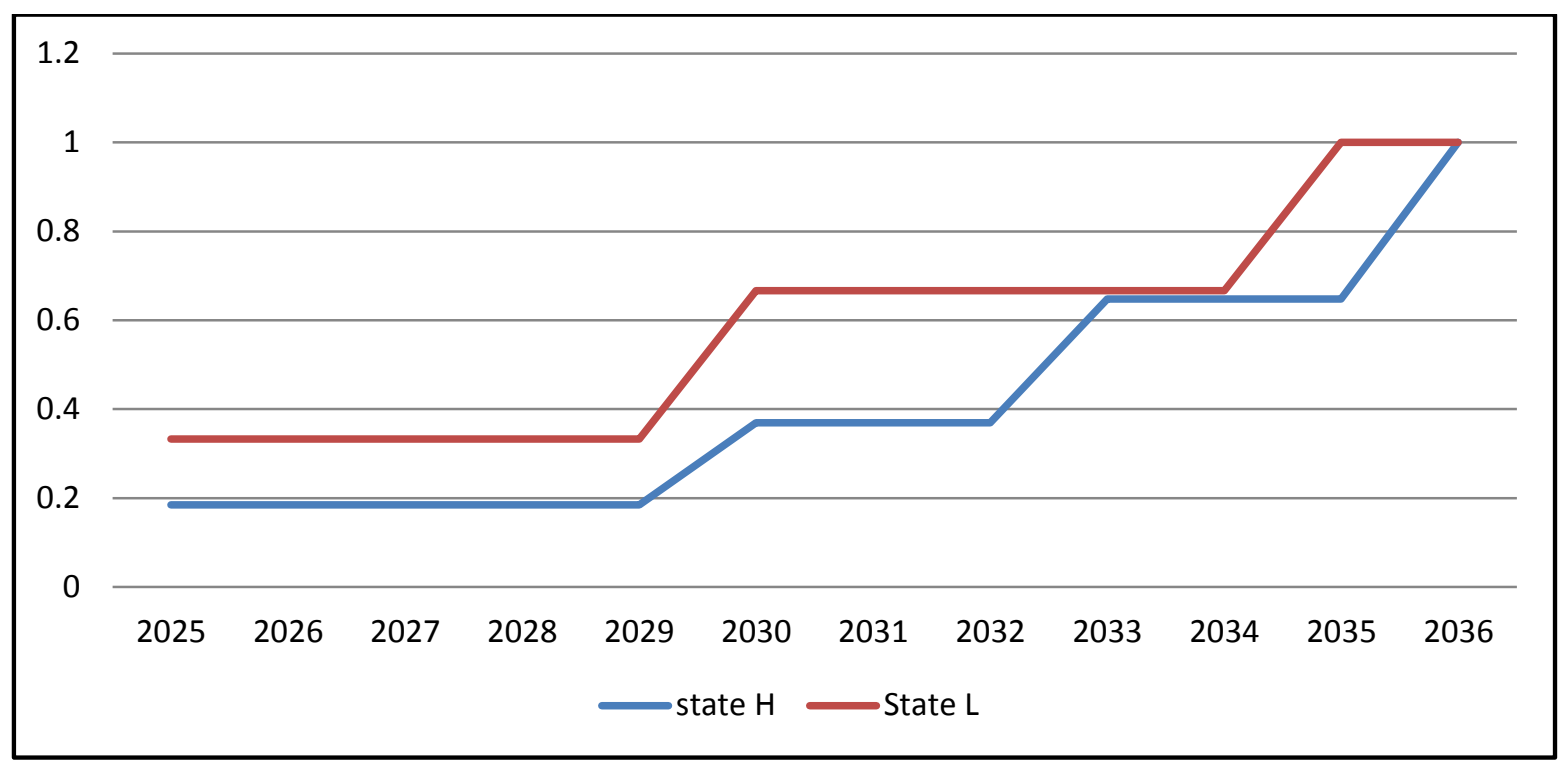

Graph 2.6 shows a comparison of CFH debt repayment schemes between two fibre uptake states (from 2025 to 2036). The horizontal axis represents the year and the vertical axis represents the cumulative portion of $\mathrm{CFH}$ debt securities that has been repaid in that particular year. Unquestionably, the repayment scheme in state $\mathrm{H}$ (over $20 \%$ uptake rate) is more "soft" than in state L. In graph 2.6, the repayment scheme at state L dominates the repayment scheme at state $\mathrm{H}$, which represents that Chorus always needs to repay the debt earlier when the fibre uptake rate is low in the future. Similar to the equity subsidy, CFH offers a higher present value debt subsidy to Chorus if the fibre demand turns out to be good.

\section{The present value calculation of CFH debt subsidies}

As for CFH's equity investment, the present value of CFH's initial debt investment in Chorus's UFB roll-out equals $\$ 429$ million. In order to calculate the present value of CFH debt securities, we discount all debt repayment cash flows back to 2013 in an annual 
discount rate of $6.67 \%$. By doing that, we get the present values of the $\mathrm{CFH}$ debt securities equal to $\$ 159.4$ million for state $\mathrm{L}$ and $\$ 141.6$ million for state $\mathrm{H}$. Accordingly, the present values of debt subsidies should equal \$269.6 million at state $\mathrm{L}$ and $\$ 287.4$ million at state $\mathrm{H}$.

Recall that, at state L, the present value of the debt subsidy equals \$269.6 million and the present value of the equity subsidy equals $\$ 293.2$ million. Therefore, the present value of total subsidies (debt subsidies and equity subsidies) should equal \$563 million at state L. On the other hand, at state $\mathrm{H}$, the present value of the debt subsidy equals $\$ 287.4$ million and the present value of the equity subsidy equals $\$ 319.2$ million, which indicates that the present value of total subsidies at this case should equal $\$ 606$ million. As we can see clearly, the amount of total subsidy is bigger if fibre demand is bigger. Therefore, by comparing the present value of subsidy to the states of UFB uptake, CFH motivates Chorus to work hard in order to have a higher demand on the UFB rollout. 


\section{Chapter 3: Literature reviews}

In a PPP project, the information asymmetry and contract incompleteness provide the private party with considerable managerial flexibility, which we study using real options analysis.

The term information asymmetry here describes the situation when information is not symmetrically allocated between two parties. That is, one of them has superior information on several important parameters relating to undertaking the project over the other. Normally, the information advantage will be allocated to the agent rather than the principal. In the example of the UFB project, Chorus may have better information about the "true" capital expenditure required to roll out a fibre network than $\mathrm{CFH}$.

In general, two kinds of consequences can be caused by information asymmetry: ex post inefficiency and ex ante inefficiency, where the terms ex post and ex ante refer to inefficiencies that arise respectively after and before forming the contract. ${ }^{21}$

The case of ex post inefficiency is that in which the agent can benefit from its superior information by pursuing its own interests at the cost of the principal, since the principal cannot verify the effort of the agent in a particular job ("hidden agenda"). In the case of the UFB project, if CFH cannot observe the capital expenditure function of Chorus, the firm may be able to "inflate" its apparent capital expenditures in order to get "extra" reimbursements from $\mathrm{CFH}$.

In contrast, if $\mathrm{CFH}$ can observe the capital expenditure function of Chorus after signing the contract, a cost-reimbursement incentive scheme (a proportional subsidy in our model) can be applied therefore the majority of conflict in our case can be eliminated. However, the application of a cost-reimbursement incentive scheme does not necessarily eliminate the conflict completely, since part of the conflict may rise from ex ante inefficiency. Therefore, in order to eliminate the conflict completely, $\mathrm{CFH}$ may need to have perfect information to pick up the best agent to undertake the UFB project (if the quality of service could be specified, the differences of agent are only on their capital expenditure functions, with complete competition, there must be a "right" agent for UFB project which can produce the global minimum conflict).

${ }^{21}$ A nice summary of this literature can be found in Laffont and Tirole(1993). 


\subsection{The literature review of PPPs in the scope of incomplete contracts}

Under a symmetric information assumption, Hart et al. (1997) set up a single stage model to compare the desirability of private and public ownership when there exists both qualityimprovement (productive) and cost reduction (unproductive) investments for the manager. Their model indicates that when the ownership is allocated to the private sector the manager will reduce costs excessively, at the expense of quality, whereas with public ownership there are blunted incentives for both cost reduction and quality improvements. Therefore, they suggest that private ownership should be the case when the quality reductions from unproductive investment can be somehow controlled through contracting and quality improvement innovations are what matter. In contrast, when cost-cutting innovations are non-contractible and have a larger adverse effect on quality, and when quality-improvement innovations do not matter, the case of nationalization should be stronger.

Except for the optimal ownership allocation, the impact of bundling various production stages is one of the main issues in the previous literatures. Hart (2003) built up a multiplestage version of the model ${ }^{22}$ in Hart et al. (1997) to study the costs and benefits of publicprivate partnerships. In his model, two production stages (both building and operating) are included in order to model the impacts of cross-stage externalities on investment incentives. In stage 0 , a builder has the flexibility to choose the level of two sorts of investments (productive and unproductive) which may both reduce the operating costs, however only the productive investment improves the service quality therefore increasing the social benefits; the unproductive investment reduces the social welfare. Under unbundling (traditional procurement), the builder cannot internalize the effect of this effort on benefits nor on costs. He exerts too little of his effort on the productive investment but the right amount on the unproductive investment. In contrast, under the case of bundling (PPPs), the builder again does not internalize the social benefits, but does internalize the costs. As a result, he implements a more appropriate level of productive investment (compared with the case of bundling), although still too little compared to the case of first-best, but also too much of the unproductive investment. Hart suggests that bundling (PPPs) is preferred over traditional

\footnotetext{
${ }^{22}$ Although Hart (2003)'s model setting is similar to that of Hart et al. (1997), several differences can be found between them. First, for simplicity, Hart (2003) ignores the choice between public and private ownership and assumes that all provision is private. Second, in order to estimate the effects of cross-stage externalities, Hart (2003) added an operation stage to the single-stage model.
} 
procurement when the unproductive investment can be well incentivized by the contract, whereas the productive investment cannot be. In contrast, when the productive investment can be well incentivized by the contract but the unproductive investment cannot, the unbundling (traditional procurement) should be the case.

Combining bundling and ownership allocation, Bennett and Iossa (2006) use a richer model to study the desirability of PPPs. The main contribution of their study to the literature is the observation that allocating ownership rights to an agent may reduce his bargaining power when there exists externalities. There are two sorts of externalities in their description. A building innovation that improves the social welfare benefit and comes with a reduced operation cost is what they call positive externality. In contrast, a building innovation that improves the social welfare benefit but comes with an increased cost at the operation stage is what they call a negative externality. Ownership gives the control right to the owner to decide whether to implement quality-enhancing or cost-cutting innovations proposed by the investor. PPPs are always preferred over traditional procurement when there exists a positive externality, and vice versa when there is a weak negative externality. Private ownership is nevertheless optimal for generic facilities with high residual value. Although their research is similar to that by Hart (2003), there are several important differences. First, a renegotiation process takes place since Bennett and Iossa (2006) assume that although both investments are unverifiable ex ante they are verifiable ex post. ${ }^{23}$ Besides, a residual stage has been added to Bennett and Iossa's (2006) model to study the explicit role of residual value. Last but not least, the ownership of assets is not a point in Hart (2003)'s model ${ }^{24}$ but plays an important role in Bennett and Iossa's (2006) model.

By allowing a compensation scheme and risk-averse agents, Martimort and Pouyet (2008) extend their model to a more complete contracting scope (both the quality of the infrastructure and operating costs are contractible, although not perfectly). Incentives and welfare are higher under a PPP project when there is a positive externality between building and operation stages compared to traditional procurement. The reverse may happen for a negative externality. Although ownership still could be used to align incentives, its effect is

\footnotetext{
${ }^{23}$ Hart (2003) assumes investments are unverifiable both ex ante and ex post.

${ }^{24}$ As we discussed before, Hart (2003) ignores the choice between public and private ownership to simplify the model.
} 
weaker ${ }^{25}$ and less important than the decision of bundling. Besides, they found that part of the benefits of bundling (PPPs) could be explained by better risk sharing.

As in much of the literature, the character of cross-stage externalities plays a huge role in determining the optimal contract between Chorus and CFH (government). The whole UFB project could be divided into three main stages- the network design stage, the network building stage and service operation stage. In the planning stage, either the government could deliver the whole integrated UFB package to Chorus or it could contract out one or two phases of the whole project to Chorus and leave the rest to another company. It seems that there exist positive externalities among the various UFB stages, which induces the government to hand over the whole package to Chorus. Besides, the ownership matters in our case since the company has some flexibility regarding the technology used to build the network. In the roll-out process, there are various technologies available to Chorus. For example, either Chorus can choose to put fibre cables on power poles or they can bury fibre cables underground. The former cuts the building cost but raises the maintenance costs which may be undesirable from the perspective of social welfare, while the latter one rises the building cost but is far easier to maintain which may enhance social welfare. ${ }^{26}$ The ownership in this case should be allocated to the private sector since, by internalizing the inter-stage externality, it induces Chorus to put more cables underground and less cables overhead therefore increasing the social welfare.

\subsection{The literature review of PPPs in the scope of real options analysis}

Flexibility allows investment decision makers to update their investment decisions as new information arrives, which helps them to take advantage of new opportunities and/or to alleviate actual or potential losses. The key idea of real options analysis is to value the managerial flexibility of real assets with the application of option concepts. The quantitative origins of real options analysis derive from the seminal work of Black and Scholes (1973) and Merton (1973) in pricing financial options. A decision-tree approach introduced by Cox, Ross, and Rubinstein (1979) highly simplifies the option valuation in discrete-time. This valuation method is the one used in this thesis. Over the years, the real option theory has been significantly developed by numerous scholars (such as Dixit and Pindyck (1994),

\footnotetext{
${ }^{25}$ That is we compare it to the more incomplete contract settings such as, Hart et al. (1997) and Bennett and lossa's (2006).

${ }^{26}$ The increase of social welfare is based on the assumption that the savings in maintenance cost dominate the savings in building cost.
} 
Trigeorgis (1996), Amram and Kulatilaka (1999), Copeland and Antikarov (2001) and Guthrie (2009)). ${ }^{27}$

Real options analysis has been applied to study the infrastructure projects (such as Ford, Lander and Voyer (2002), Garvin and Cheah (2004) and Grimes (2011)). In the general real option literature, real options analysis is considered to be applicable if the following criteria are met. First, the investment is at least partly irreversible, which is normally true for the project involving specified infrastructure investment (in the UFB roll-out, a large portion of infrastructure and device investment are technology specific, which is definitely not full recoverable). Second, the project should involve uncertainty which may eventually affect the project's net present value (in the UFB case, the net present value of the project is affected by both demand uncertainty and cost uncertainty). Third, the cost to utilise flexibility cannot be too high. For example, if abandonment is too costly, it is unlikely that the firm will exercise the abandonment option, which renders the flexibility to abandon of little value. In the UFB project, the associated costs for Chorus to defer or abandon the infrastructure construction are limited.

Over the past two decades, many researchers have applied real options analysis to conduct evaluation of PPP projects. Rose (1998) uses Monte Carlo simulation to estimate the values of two separate options (the first option entitles the government to reduce the "concession period" and the second one grants the concessionaire the deferral of the "concession fee" to the government), and more important, the value of their "interacting effect" in the Melbourne CityLink Project. Focusing on the same project (Melbourne CityLink Project), Alonso-Conde, Brown and Rojo-Suarez (2007) not only estimate the values of relevant options but also highlight a non-monotonic relationship between these options and changes in volatility. Liu and Cheah (2009) value both a guarantee (the government guarantees the minimum level of revenue flow to the concessionaire) and a cap (the government caps the highest level of return for the concessionaire) in a wastewater treatment plant located in Southern China through Monte Carlo simulations. As a result of their valuation, the negotiation bands for both the initial transfer price (with a fixed normal tariff) and the normal rate of tariff (with a fixed transfer price) could be created. Shan, Garvin and Kumar (2010) illustrate how to determine the strike prices of a collar option (which combines a call and put option) in a real toll PPP transportation project. Liu, Yu and Cheah (2014) evaluate

${ }^{27}$ A nice summary about the real option literature could be found in Trigeorgis (1993). 
the guarantee of restrictive competition (an option that the concessionaire may require the government to limit similar projects in the same area of the original infrastructure project) in a PPP highway project in Nanjing, China.

Using real options analysis, some authors focus on the incentive scheme design that aligns the public and private interests in a PPP project. Rocha Armada, Pereira and Rodrigues (2012) present four kinds of incentive schemes (an investment subsidy that reduces the investment cost, a revenue subsidy that increases the value of the underlying cash flows, a minimum demand guarantee and a rescue option that reduce the demand uncertainty) that could be optimally arranged by the government to induce the concessionaire to invest early on PPPs infrastructure projects. ${ }^{28}$ Although the net costs of these incentive schemes are always negative and equal to the value of the option to defer, they produce different time patterns of the cash flows for the government, which imply that the choice of the types of incentives may be determined by the degree of government commitment to future generations of taxpayers. Besides, they show that a rescue option that allows the concessionaire to recover a fraction of the initial investment through abandoning the project is not common in PPPs due to its extremely high fraction of recovery requirement for certain demand levels. ${ }^{29}$ Chen and Qin (2012) suggest that a real option incentive scheme (which is combined by a continuation option and an abandonment option) could be offered by the government to the concessionaire to improve revenue management in PPP projects. More interesting, they show that not only the premium of the real option incentive scheme can finance possible capacity expansions but also the real option incentive scheme can motivate the concessionaire to increase the investment in quality improvement.

The previous real option studies mainly focused on the evaluation of managerial flexibility in PPP projects. Although the incentive schemes and the concessionaire's investment behaviors have been studied by some people, the decision flexibility featured in their models is very limited, and more important, the decision flexibility has never been granted to the government, ${ }^{30}$ which may not be theoretically optimal.

\footnotetext{
${ }^{28}$ They assume that from the government's perspective, it is always optimal to invest immediately. Therefore, the conflict increases only when the concessionaire has an incentive to delay investment.

${ }^{29}$ They claim the fraction recovered needs to be significantly higher than $100 \%$ for certain demand levels ${ }^{30}$ In Rocha Armada, Pereira and Rodrigues (2012)'s study, they simply assume the government always prefers immediate investment and the private concessionaire can only decide the timing of investment. In Chen and Qin (2012)'s study, the government's decision on capacity remains unchanged over time.
} 


\section{Chapter 4: The structure for basic model}

\subsection{The objective functions}

In this thesis, one of the main research goals is identifying the potential conflicts between public and private parties. Since conflicts may arise if different parties have different economic interests, we need to specify both parties' objective functions. In the UFB project, as a private sector manufacturer, Chorus may try to maximize the present value of its economic profits and therefore will care about only the producer surplus (PS) and the capital expenditures of the UFB project. $\mathrm{CFH}$, as a representative of government, will care about the present value of total surplus (customer surplus plus the producer surplus) and the capital expenditures instead since the social welfare maximization should be its main consideration. ${ }^{31}$

For a standard telecommunication project, a large amount of capital expenditure normally occurs in the infrastructure construction period, and compared to the revenue, the operating cost to run the facility is negligible. Therefore, in any post-construction point, we may assume that both construction and operational expenditures are zero. (The construction cost is omitted as a sunk cost for post-construction period, while the operating cost is omitted due to its size relative to revenue.)

For a given linear demand function, what should be the specified function of customer surplus and producer surplus?

At graph 4.1, the fibre contract price is $\hat{\boldsymbol{p}} .^{32}$ The red triangle area CDE is the consumer surplus flow at date $\boldsymbol{n}$ when there have been $\boldsymbol{i}$ down moves and $\boldsymbol{n}-\boldsymbol{i}$ up moves in the binomial tree that describes how demand changes over time, which we label as $\boldsymbol{V}(\boldsymbol{i}, \boldsymbol{n})$. The blue rectangle area ABCD is the producer surplus flow at date $\boldsymbol{n}$ when there have been $\boldsymbol{i}$ down moves and $\boldsymbol{n}-\boldsymbol{i}$ up moves, which we label as $\boldsymbol{X}(\boldsymbol{i}, \boldsymbol{n})$.

\footnotetext{
${ }^{31}$ To simplify, we make an assumption that principal-agent problem does not exist between $\mathrm{CFH}$ and government therefore $\mathrm{CFH}$ is the social planner.

${ }^{32}$ To simplify, we assume the fibre contract price $\hat{P}$ is constant over time. This assumption is quite realistic since the fibre contract prices specified in the UFB project do not change until 2015. Besides, even though the prices of different fibre service packages are going to be changing after 2015 , the average price is constant over time.
} 


\section{Graph 4.1: The functions for demand and supply flows of the UFB Project}

Fibre contract price $\widehat{\boldsymbol{p}}$

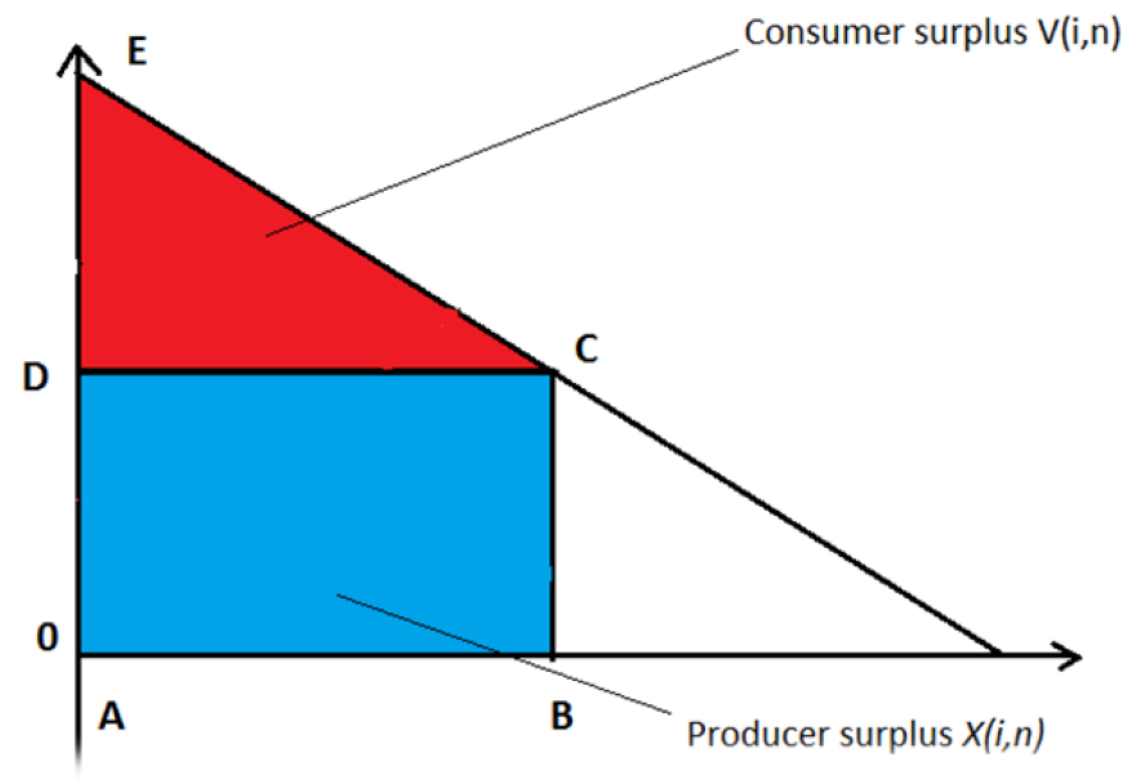

We describe the linear demand curve as $\boldsymbol{p}=\boldsymbol{x}-\boldsymbol{c} \boldsymbol{q}$ where $\boldsymbol{c}$ is a constant and $\boldsymbol{x}$ is a demand driver that changes over time. Therefore, the level of demand $\widehat{\boldsymbol{q}}(\boldsymbol{x})$ satisfies

$$
x-c \widehat{q}(x)=\widehat{p} \leftrightarrow \widehat{q}(x)=\frac{x-\widehat{p}}{c} .
$$

According to the zero operating costs assumption, the producer surplus flow is

$$
X(i, n)=\widehat{p} \cdot \widehat{\boldsymbol{q}}(\boldsymbol{x})=\frac{\widehat{\boldsymbol{p}}(\boldsymbol{x}-\widehat{\boldsymbol{p}})}{\boldsymbol{c}} .
$$

The consumer surplus is

$$
V(i, n)=\int_{0}^{\widehat{q}(x)}(x-c q-\widehat{p}) d q=\left[\left(x-\widehat{p} \cdot q-\frac{c}{2} q^{2}\right)\right]_{0}^{\widehat{q}(x)}=\frac{(x-\widehat{p})^{2}}{2 c}
$$

If we keep the fibre contract price $\widehat{\boldsymbol{p}}$ constant, then

$$
X(i, n)=\frac{\widehat{p}(x-\widehat{p})}{c} \text { and } V(i, n)=\frac{(x-\widehat{p})^{2}}{2 c}
$$

This implies that

$$
\frac{\text { consumer surplus flow }}{\text { producer surplus flow }}=\frac{V(i, n)}{X(i, n)^{2}}=\frac{\frac{(x-\widehat{p})^{2}}{2 c}}{\left[\frac{\widehat{p}(x-\widehat{p})}{c}\right]^{2}}=\frac{c}{2 p^{2}}(1) .
$$


This constant ratio guarantees that $\boldsymbol{V}(\boldsymbol{i}, \boldsymbol{n})$ is proportional to the square of $\boldsymbol{X}(\boldsymbol{i}, \boldsymbol{n})$, which simplifies the modelling for the consumer surplus. In the following sections, we use the constant ratio $\frac{c}{2 p^{2}}$ to measure the relative important of consumer and producer surpluses, which we label as Con.

\subsection{Model cities}

In our model, four cities have been formed to study the differences of choices between Chorus and CFH. The demand and construction expenditure are the features we use to make cities different. Specifically, we apply a demand multiplier $(\boldsymbol{D R})$ to the producer surplus of a standard city to form a high demand city, while we apply a cost multiplier $(\boldsymbol{C R})$ to the construction expenditure of a standard city to form a high cost city. $\boldsymbol{D} \boldsymbol{R}$ may be sourced from the difference of population density across the UFB coverage area, while $\boldsymbol{C R}$ may be sourced from the different fibre deployment technology that could be applied across the UFB coverage area. The details of the four cities are provided below.

\section{City HL (High demand and low capital expenditure):}

This city has high demand on the fibre network and the expenditure needed to build the fibre network is low. At date $\boldsymbol{n}$, when there are $\boldsymbol{i}$ down moves, the per-period flow of producer surplus (that is, revenue) of this city will equal $\boldsymbol{D R} \times \boldsymbol{X}(\boldsymbol{i}, \boldsymbol{n})$. However, for CFH, the perperiod flow of total surplus will equal $\left[\boldsymbol{D R} \times \boldsymbol{X}(\boldsymbol{i}, \boldsymbol{n})+\boldsymbol{D} \boldsymbol{R}^{\mathbf{2}} \times \boldsymbol{V}(\boldsymbol{i}, \boldsymbol{n})\right] .^{33}$

\section{City HH (High demand and high capital expenditure):}

This city has high demand on the fibre network and the expenditure needed to build the fibre network is high. At date $\boldsymbol{n}$, when there are $\boldsymbol{i}$ down moves, the per-period flow of producer surplus of this city will equal $\boldsymbol{D R} \times \boldsymbol{X}(\boldsymbol{i}, \boldsymbol{n})$. However, for $\mathrm{CFH}$, the per-period flow of total surplus will equal $\left[D R \times X(i, n)+D R^{2} \times V(i, n)\right]$.

\section{City LL (Low demand and low capital expenditure):}

This city has low demand on the fibre network and the expenditure needed to build the fibre network is low. At date $\boldsymbol{n}$, when there are $\boldsymbol{i}$ down moves, the per-period flow of producer surplus of this city will equal $\mathbf{1} \times \boldsymbol{X}(\boldsymbol{i}, \boldsymbol{n})$. However, for $\mathrm{CFH}$, the per-period flow of total surplus will equal $1 \times[X(i, n)+V(i, n)]$.

${ }^{33}$ This is consistent with equation 1. That is, $\frac{\text { consumer surplus flow for a high demand city }}{\text { producer surplus flow for a high demand city }{ }^{2}}=\frac{D R^{2} \times V(i, n)}{[D R \times X(i, n)]^{2}}=\frac{V(i, n)}{X(i, n)^{2}}$. 


\section{City LH (Low demand and high capital expenditure):}

This city has low demand on the fibre network and the expenditure needed to build the fibre network is high. At date $\boldsymbol{n}$, when there are $\boldsymbol{i}$ down moves, the per-period flow of producer surplus of this city will equal $\mathbf{1} \times \boldsymbol{X}(\boldsymbol{i}, \boldsymbol{n})$. However, for $\mathrm{CFH}$, the per-period flow of total surplus will equal $\mathbf{1} \times[\boldsymbol{X}(\boldsymbol{i}, \boldsymbol{n})+\boldsymbol{V}(\boldsymbol{i}, \boldsymbol{n})]$.

The above descriptions specify the revenue function for each city. However, before we can go further, we need to define our cost function for the basic model. Let $\operatorname{Cost}\left(\boldsymbol{j}_{a}, \boldsymbol{j}_{\boldsymbol{b}}, \boldsymbol{j}_{c}, \boldsymbol{j}_{\boldsymbol{d}}\right)$ denote the construction expenditure in each construction period, where

$$
\operatorname{Cost}\left(j_{a}, j_{b}, j_{c}, j_{d}\right)=C \times\left(j_{a}+j_{c}+C R\left(j_{b}+j_{d}\right)\right)^{2}
$$

$\boldsymbol{j}_{a}, \boldsymbol{j}_{\boldsymbol{b}}, \boldsymbol{j}_{\boldsymbol{c}}$ and $\boldsymbol{j}_{\boldsymbol{d}}$ represents how many construction periods are going to be finished for each city in this period, where the subscript $\boldsymbol{a}, \boldsymbol{b}, \boldsymbol{c}$ and $\boldsymbol{d}$ stand for city HL, city HH, city LL and city LH respectively. In our basic model, there is only one construction period for building each city, therefore the values of $\boldsymbol{j}_{\boldsymbol{a}}, \boldsymbol{j}_{\boldsymbol{b}}, \boldsymbol{j}_{\boldsymbol{c}}$ and $\boldsymbol{j}_{\boldsymbol{d}}$ here will be either 0 or 1 . The marginal cost function is increasing over the number of cities to be built. For example, if we only build a low cost city $(H L$ or $L L), \operatorname{Cost}(\mathbf{1}, \mathbf{0}, \mathbf{0}, \mathbf{0})=\operatorname{Cost}(\mathbf{0}, \mathbf{0}, \mathbf{1}, \mathbf{0})=\boldsymbol{C}$, or if we only build a high cost city $(\mathrm{HH}$ or $\mathrm{LH}), \boldsymbol{C o s t}(\mathbf{0}, \mathbf{1}, \mathbf{0}, \mathbf{0})=\boldsymbol{C o s t}(\mathbf{0}, \mathbf{0}, \mathbf{0}, \mathbf{1})=\boldsymbol{C} \times(\boldsymbol{C} \boldsymbol{R})^{2}$. However, if we build up a high cost city and a low cost city together, $\operatorname{Cost}(1,1,0,0)=\operatorname{Cost}(0,0,1,1)=\operatorname{Cost}(1,0,0,1)=$ $\operatorname{Cost}(0,1,1,0)=C \times(1+C R)^{2}$, where $C \times(1+C R)^{2}=C \times\left(1+C R^{2}+2 C R\right)>C \times\left(1+C R^{2}\right)$ (the case that two cities have been built separately) due to the existence of the extra term $\mathbf{2 C R} \times \boldsymbol{C}$. The extra term in the inequality explains the source of increasing marginal cost. In reality, this extra term may come from the various constraints that the party faces. For example, if there is a limited local labour market in this country, the party may incur an extra cost to build up two cities together rather than building them up separately. This is because, when facing a limited local labour market, the firm may need to hire someone else from overseas to join the construction, which often incurs additional cost. The cases of building up three cities and four cities together are quite similar to our two cities case.

\subsection{The model setting-up}

The government (CFH) gives a firm (Chorus) a PPP contract that allows the private firm to build up the UFB network in four nominated cities. At each period, the firm has flexibilities to choose both which of the cities it wants to build and when it wants to build them. In the version of the model considered in this chapter, all construction required to roll out fibre to a 
single city can be completed in one period. We will see that this significantly simplifies the analysis. Construction costs $\boldsymbol{C}$ to roll out fibre to a single standard city and $\boldsymbol{C} \times \boldsymbol{C R}^{2}$ to roll out fibre to a single high cost city. Multiple cities can be completed in a single period, but the firm incurs extra construction costs. The whole UFB project can be suspended or resumed without cost. However, the development right expires after $\boldsymbol{T}$ years.

There are two state variables in our model. ${ }^{34}$ The first state variable, $\boldsymbol{X}(\boldsymbol{i}, \boldsymbol{n})$, denotes a single period revenue for a standard city at date $\boldsymbol{n}$ if exactly $\boldsymbol{i}$ down moves and $\boldsymbol{n}-\boldsymbol{i}$ up moves have occurred since date $0 .{ }^{35}$ Equation 2 is the formula we use to generate the state variable $X(i, n)$.

$$
X(i, n)=X_{0} \times D_{0}^{i} \times U_{0}^{n-i}(2)
$$

The $\boldsymbol{T}=6$ year lifetime of the UFB construction period is divided into a sequence of $\boldsymbol{N}=\mathbf{1 2}$ periods in our basic model. Therefore the time measure for each step $\boldsymbol{d} \boldsymbol{t}=\frac{\boldsymbol{T}}{\boldsymbol{N}}=\mathbf{0 . 5}$ equals $\boldsymbol{Y}$ (which is the time to build up one city measured by year) in our basic model. However, we will have the smaller time step $\boldsymbol{d} t$ to make our general model more accurate. $\mathbf{D}_{\mathbf{0}}$ is a constant growth factor when there has been one down move during this period, while $\mathbf{U}_{\mathbf{0}}$ is a constant growth factor when there has been one up move during this period. The starting value $\boldsymbol{X}_{\mathbf{0}}$ is the revenue for this standard city today (at date 0 ).

The second state variable, $\boldsymbol{V}(\boldsymbol{i}, \boldsymbol{n})$, denotes a single period consumer surplus for a standard city at date $\boldsymbol{n}$ if exactly $\boldsymbol{i}$ down moves and $\boldsymbol{n}-\boldsymbol{i}$ up moves have occurred since date 0 . Similarly, we have equation 3 for $\boldsymbol{V}(\boldsymbol{i}, \boldsymbol{n})$.

$$
V(i, n)=C S_{0} \times D_{0}{ }^{2 i} \times U_{0}{ }^{2(n-i)}
$$

The starting value $\boldsymbol{C} \boldsymbol{S}_{\mathbf{0}}$ is the consumer surplus for this standard city at the date 0 , which equals $\boldsymbol{C o n} \times \boldsymbol{X}_{\mathbf{0}}{ }^{2} \cdot \boldsymbol{D}_{\mathbf{0}}{ }^{2}$ and $\boldsymbol{U}_{\mathbf{0}}{ }^{2}$ are the constant growth factor squares.

Besides, there are several important parameters. $\boldsymbol{\sigma}$ is the annual standard deviation of the state variable, which we used to model the volatility. $\boldsymbol{R}_{\boldsymbol{f}}$ is the sum of 1 and the risk free rate, which we use for discounting the cash flow in each period. In order to apply the risk neutral pricing formula, $\pi_{\boldsymbol{u}}$ and $\boldsymbol{\pi}_{\boldsymbol{d}}$ have been introduced here as the risk neutral probabilities of up and down moves occurring in the next period respectively, where $\boldsymbol{\pi}_{\boldsymbol{u}}=\frac{\boldsymbol{K}-\boldsymbol{D}_{\mathbf{0}}}{\boldsymbol{U}_{\mathbf{0}}-\boldsymbol{D}_{\mathbf{0}}}$ and $\boldsymbol{\pi}_{\boldsymbol{d}}=\mathbf{1}-$

\footnotetext{
${ }^{34}$ According to our setting in section 4.1, there is only one independent state variable since $\boldsymbol{X}(\boldsymbol{i}, \boldsymbol{n})$ and $\boldsymbol{V}(\boldsymbol{i}, \boldsymbol{n})$ are perfectly correlated.

${ }^{35}$ The standard areas we mean here are those areas with standard demand for UFB, in contrast to High demand areas, where the demand for UFB is higher than the standard area.
} 
$\boldsymbol{\pi}_{\boldsymbol{u}} \cdot{ }^{36} \boldsymbol{K}$ is the sum of 1 and the annual risk-adjusted growth rate. ${ }^{37}$ The parameter $\boldsymbol{P V P S}=$ $\frac{R_{f} \times X_{0}}{R_{f}-K}$, is the present value of the perpetual revenue stream of $X(i, n), \operatorname{starting}$ at $X(0,0)=$ $\boldsymbol{X}_{\mathbf{0}} \cdot{ }^{38}$ The parameter $\boldsymbol{P V C S}=\frac{\boldsymbol{R}_{\boldsymbol{f}} \times \boldsymbol{C} \boldsymbol{S}_{\mathbf{0}}}{\boldsymbol{R}_{f}-\boldsymbol{K}\left(\pi_{u} D_{0}{ }^{2}+\pi_{\boldsymbol{d}} \boldsymbol{U}_{0}{ }^{2}\right)}$, is the present value of the perpetual consumer surplus cash flow stream of $\boldsymbol{V}(\boldsymbol{i}, \boldsymbol{n})$, starting at $\boldsymbol{V}(\mathbf{0}, \mathbf{0})=\boldsymbol{C} \boldsymbol{s}_{\mathbf{0}}{ }^{39}$ The baseline values of all our parameters are reported at table 4.1 .

\section{Table 4.1: Parameter values for the basic model}

\begin{tabular}{|c|c|c|}
\hline Parameter & Symbol & Value \\
\hline \multicolumn{3}{|l|}{ a) Real world parameter } \\
\hline Annual demand Volatility & $\sigma$ & 0.15 \\
\hline Present value for $\boldsymbol{X}_{0} *$ & PVPS & 8.5 \\
\hline Present value for $\boldsymbol{C} \boldsymbol{S}_{0} *$ & PVCS & 5.7 \\
\hline Total capital expenditure* & $C$ & 10 \\
\hline Expected growth rate & $\mu$ & 0.07 \\
\hline Annual risk-free interest rate & $r_{f}$ & 0.015 \\
\hline UFB contract duration (measured by year) & $T$ & 6 \\
\hline UFB contract period & $N$ & 12 \\
\hline Cost ratio & $C R$ & 2 \\
\hline Demand ratio & $D R$ & 3 \\
\hline $\begin{array}{l}\text { the constant multiplier between consumer and } \\
\text { producer surpluses }\end{array}$ & Con & 4 \\
\hline \multicolumn{3}{|l|}{ b) Model parameter } \\
\hline Time for each period & $d t$ & 0.5 \\
\hline Time to build up one city (measured by year) & $\boldsymbol{Y}$ & 0.5 \\
\hline Per-period risk adjusted growth factor & $\boldsymbol{K}$ & 0.993 \\
\hline Per-period growth factor (up) & $\boldsymbol{U}_{\mathbf{0}}$ & 1.112 \\
\hline Per-period growth factor (down) & $D_{0}$ & 0.899 \\
\hline Per-period risk-neutral probability (up) & $\pi_{u}$ & 0.438 \\
\hline
\end{tabular}

\footnotetext{
${ }^{36}$ For more detail see chapter 3, Guthrie (2009).

${ }^{37}$ In our model, when calculating present values of cash flow streams we adjust for risk via the numerator rather than the denominator. $\boldsymbol{K}$ here is the risk-adjusted growth factor which we use to adjust for risk. It differs from the actual growth factor $\mathbf{1}+\boldsymbol{g}$ by including a risk-adjusted effect, which normally makes it smaller than the actual growth factor. After multiplying the expected cash flow by the risk-adjusted growth factor $\mathrm{K}$, the risky cash flow could be treated as a risk-free cash flow, therefore a risk-free discount rate could be applied to discount the expected cash flow, where $K=\frac{1+g}{\boldsymbol{R}_{f}(\mathbf{1}+\boldsymbol{\rho})}$.

${ }^{38}$ Chorus receive a perpetual cash flow $\boldsymbol{X}(\boldsymbol{i}, \boldsymbol{n})$ from a completed standard city in each period, where $\boldsymbol{X}_{\mathbf{0}}$ is the initial value of $\boldsymbol{X}(\boldsymbol{i}, \boldsymbol{n})$.

${ }^{39} \mathrm{CFH}$ receive a perpetual cash flow $\boldsymbol{V}(\boldsymbol{i}, \boldsymbol{n})$ from a completed standard city in each period, where $\boldsymbol{C} \boldsymbol{S}_{\mathbf{0}}$ is the initial value of $\boldsymbol{V}(\boldsymbol{i}, \boldsymbol{n})$.
} 


\begin{tabular}{lll}
\hline Per-period risk-neutral probability (down) & $\boldsymbol{\pi}_{\boldsymbol{d}}$ & 0.562 \\
Current revenue* & $\boldsymbol{X}_{\mathbf{0}}$ & 0.127 \\
Current consumer surplus* & $\boldsymbol{C S}_{\mathbf{0}}$ & 0.064 \\
\hline Per-period risk-free interest factor & $\boldsymbol{R}_{\boldsymbol{f}}$ & 1.008 \\
*These values are only for a standard city & & \\
\hline
\end{tabular}

\subsection{Decision tree}

A decision tree has been constructed in graph 4.2 in order to analyse our basic model. At date 0 , Chorus has completed no cities (category A, which is the yellow rectangle in graph 4.2) and must choose between constructing a network in one or more cities and waiting. If the firm chooses to construct one or more networks at date 0 , then it needs to choose one specified decision from 15 construction choices. ${ }^{40}$ We can see from graph 4.2 , at date 1 , these 15 construction choices will make the firm into four different state categories - four construction choices (therefore four states in date 1) are in category $\mathbf{B}$ (to build one city between date 0 and 1 , which is the orange rectangle in graph 4.2), six construction choices (therefore six states in date 1) are in category $\mathbf{C}$ (to build two cities between date 0 and 1 , which is the green rectangle in graph 4.2), four construction choices (therefore four states in date 1) are in category $\mathbf{D}$ (to build three cities between date 0 and 1 , which is the blue rectangle in graph 4.2) and one construction choice (therefore one state in date 1) is in the category $\mathbf{E}$ (to build four cities between date 0 and 1, which is the purple rectangle in graph 4.2). According to its adopted construction policy, the firm pays the corresponding costs immediately and receives the corresponding revenues from one period later onwards. At date 1, the firm will have the right to develop the remaining cities, but the remaining lifetime of the contract will be one period shorter. If, instead, the firm chooses to wait, then at date 1 it will be in category $\mathbf{A}$ and still have the right to develop all four cities, and again the remaining lifetime of the contract will be one period shorter.

\footnotetext{
${ }^{40}$ There will be $4^{2}$ choices for the firm since we have four different cities and two available decisions for each city. However, we exclude the option of waiting thereby only $4^{2}-1=15$ decision left.
} 
Graph 4.2: Decision tree for the basic model

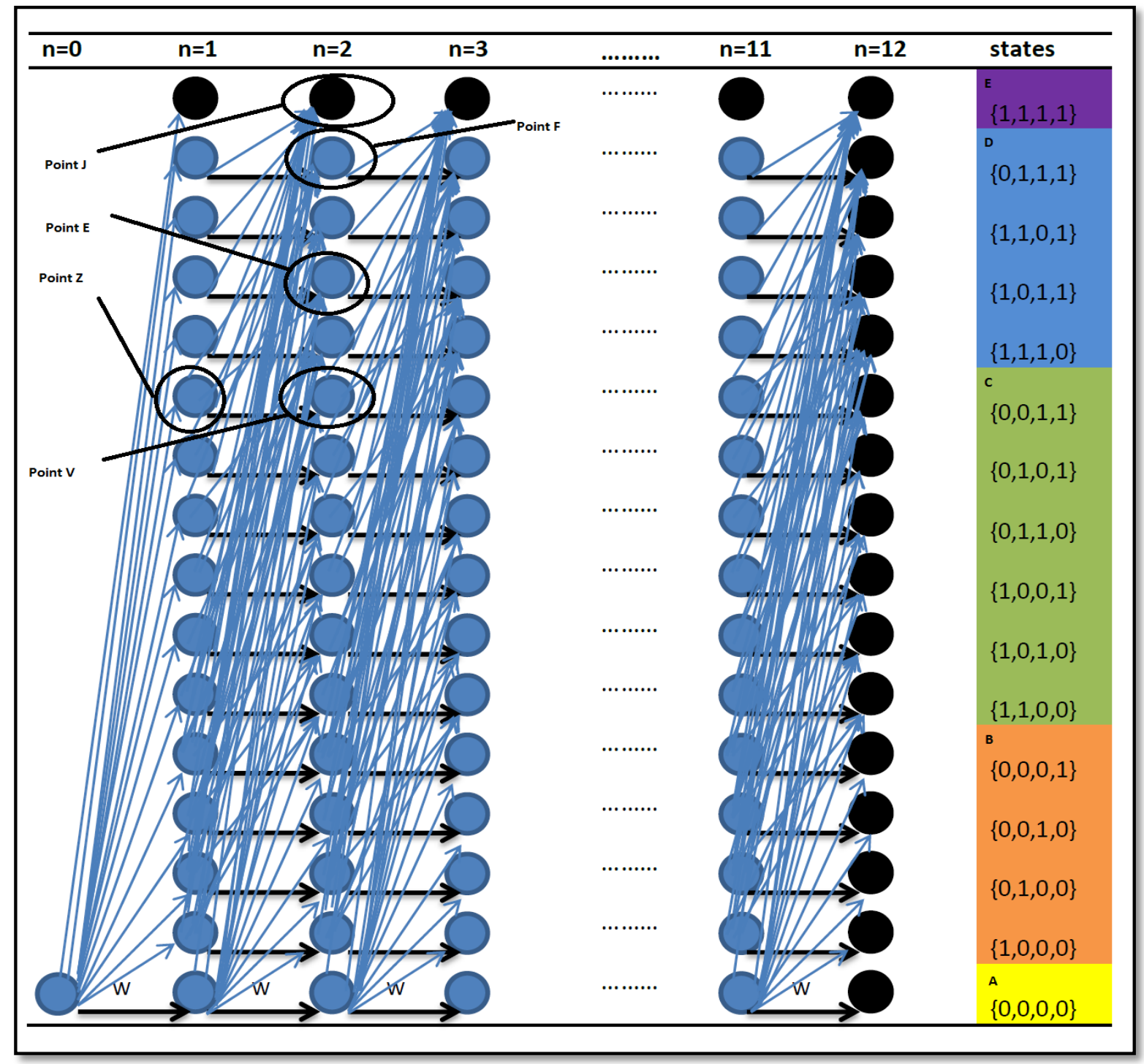

Therefore, the project will be in one of sixteen states at date 1, depending on what policy has been adopted by Chorus at date 0 . The firm reaches the terminal node and the contract ends if Chorus finishes all four cities (which is category $\mathbf{E}$ ). Otherwise, the firm must be in one of the four following state categories in date 1 and it needs to choose between constructing and waiting again.

State category D (Shaded with blue colour): There is only one remaining city for the firm to develop now since three have been finished in the past. Therefore, there are four different states in this category. Which state the firm faces in this category, depends on which of those three cities have been finished in the past. No matter which state the firm is in, now, the firm must choose either to build up the last city or to wait. 
State category C (Shaded with green colour): There are two remaining cities for the firm to develop now since the other two have been finished. Therefore, there are six different states in this category. Which state the firm faces in this category depends on which of those two cities have been finished. No matter which state the firm is in, now, the firm must choose from building up the last two cities, building up one city or waiting. The choice of building up one city has two different policies available.

State category B (Shaded with orange colour): There are three remaining cities for the firm to develop now since only one has been finished. Therefore, there are four different states in this category. Which states the firm faces in this category, depends on which city has been finished. No matter which state the firm is in, the firm must choose from building the last three cities, building two cities, building one city or waiting. Building up two cities and building up one city each has three different policies available.

State category A (Shaded with yellow colour): There are four remaining cities for the firm to develop now since no city has been finished yet. Therefore, there is only one state in this category. The firm must choose from building all four cities, building three cities, building two cities, building one city or waiting. Building up two cities and building up one city each has four different policies available, building up four cities and waiting each has one policy available, building up two cities has six different policies available. In total, there will be sixteen different policies available for this state.

If Chorus chooses to construct in the above states, the firm pays the corresponding costs at date 1 and starts to receive the corresponding revenues one period later, depending on which state category it is and which policy it adopts. At date 2, the firm will have the right to develop the remaining cities, but the remaining lifetime of the contract will be one period shorter. If, instead, the firm chooses to wait, then at date 2 it will still be where it was, with the same development right as before, and again the remaining lifetime of the contract will be one period shorter.

This argument could be applied to further dates as well, so our decision tree continues to evolve in this way until either of two situations happens: the development right expires, or construction in all four cities is completed. At that point no more decisions need to be made by the firm and all future cash flows can be valued without the need for decision trees. 
To make our decision tree clearer, we describe a specified case that the firm is in the state $\{\mathbf{0 , 0}, \mathbf{1}, \mathbf{1}\}$ at date 1 . That is, it has already completed city LL and LH. This will be the point $\mathrm{Z}$ in our graph 4.2. At that node, there are four remaining construction decisions for the firm to choose from- $-\{\mathbf{1}, \mathbf{1}, \mathbf{0}, \mathbf{0}\},\{\mathbf{1}, \mathbf{0}, \mathbf{0}, \mathbf{0}\},\{\mathbf{0}, \mathbf{1}, \mathbf{0}, \mathbf{0}\}$ and $\{\mathbf{0 , 0}, \mathbf{0}, \mathbf{0}\}$. If the firm chooses $\{\mathbf{1}, \mathbf{1}, \mathbf{0}, \mathbf{0}\}$, it is going to finish city HL and city HH in the next period, moving it to the terminal state $\{\mathbf{1}, \mathbf{1}, \mathbf{1}, \mathbf{1}\}$ at date 2 (point $\mathbf{J}$ in graph 4.2). If the firm chooses $\{\mathbf{1}, \mathbf{0}, \mathbf{0}, \mathbf{0}\}$, it is going to finish city HL and will be in state $\{\mathbf{1}, \mathbf{0}, \mathbf{1}, \mathbf{1}\}$ in date 2 (point $\mathbf{E}$ in graph 4.2). If the firm chooses $\{\mathbf{0}, \mathbf{1}, \mathbf{0}, \mathbf{0}\}$, it is going to build city $\mathrm{HH}$ and will be in state $\{\mathbf{0}, \mathbf{1}, \mathbf{1}, \mathbf{1}\}$ in date 2 (point $\mathbf{F}$ in graph 4.2). In contrast, if the firm chooses $\{\mathbf{0}, \mathbf{0}, \mathbf{0}, \mathbf{0}\}$ (waiting), no construction will happen and the firm will stay at state $\{\mathbf{0}, \mathbf{0}, \mathbf{1}, \mathbf{1}\}$ in date 2 (point $\mathbf{V}$ in graph 4.2).

\subsection{Valuation process}

In this section, we are going to describe how to calculate the present values of the UFB contract. As the decision tree in graph 4.2 makes clear, excluding the state $\{\mathbf{1}, \mathbf{1}, \mathbf{1}, \mathbf{1}\})$, we need to build a present value tree for each state. There are fifteen uncompleted states therefore fifteen present value trees in each of our valuation processes. Since we are interested in identifying the potential conflicts between Chorus and $\mathrm{CFH}$, we need to estimate not only the present values of the UFB contract for both parties under their own optimal policies (Chorus and $\mathrm{CFH}$ ), but also the present value of the UFB contract for $\mathrm{CFH}$ under Chorus' optimal policy. Therefore, we separate this section into three parts.

\subsubsection{Valuation process for Chorus (Chorus optimal policy)}

Let $\boldsymbol{F}(\boldsymbol{i}, \boldsymbol{n},\{\boldsymbol{a}, \boldsymbol{b}, \boldsymbol{c}, \boldsymbol{d}\})$ denotes the present value of the UFB contract for Chorus at the node $(\boldsymbol{i}, \boldsymbol{n})$ when the firm is in state $\{\boldsymbol{a}, \boldsymbol{b}, \boldsymbol{c}, \boldsymbol{d}\}$, where $\boldsymbol{a}, \boldsymbol{b}, \boldsymbol{c}$ and $\boldsymbol{d}$ equal the numbers of construction periods that have been completed in HL, HH, LH and LL cities respectively. Since all the cities in our basic model take one period to complete, the values of $\boldsymbol{a}, \boldsymbol{b}, \boldsymbol{c}$ and $\boldsymbol{d}$ will be either 0 or 1 , where 1 means that the city has been finished and vice versa for 0 . For example, $\boldsymbol{F}(\mathbf{0}, \mathbf{0},\{\mathbf{1}, \mathbf{1}, \mathbf{0}, \mathbf{0}\})$ represents the present value of the UFB contract for Chorus at the node $(\mathbf{0}, \mathbf{0})$ when the firm has already finished both the HL and HH cities.

If no city has been completed at the expiry date $N$ then the UFB contract has no value, so that

$$
F(i, N,\{0,0,0,0\})=0
$$


at all nodes $(\boldsymbol{i}, \boldsymbol{N})$.

Now consider the more general case where either Chorus has built up a fibre network in some cities or it has not started yet. From date $\boldsymbol{N}$ onwards the development right is worthless since the right to develop has expired. However, the firm still receives revenue from those completed cities in each post $N$ period. Therefore

$$
\begin{gathered}
F(i, N,\{a, b, c, d\})=\frac{R_{f} R(i, N,\{a, b, c, d\})}{R_{f}-K}, 41 \\
\text { where } R(i, N,\{a, b, c, d\})=[D R(a+b)+(c+d)] X(i, n)(4)
\end{gathered}
$$

for all $i=0,1, \ldots, N$. At each earlier date, Chorus must choose between constructing and waiting. If it decides to wait, then there is cash flow from cities already completed at date $n$, and at date $\boldsymbol{n}+\mathbf{1}$ the present value of the fibre contract will be $\boldsymbol{F}(\boldsymbol{i}, \boldsymbol{n}+\mathbf{1},\{\boldsymbol{a}, \boldsymbol{b}, \boldsymbol{c}, \boldsymbol{d}\})$ if an up move occurs and $\boldsymbol{F}(\boldsymbol{i}+\mathbf{1}, \boldsymbol{n}+\mathbf{1},\{\boldsymbol{a}, \boldsymbol{b}, \boldsymbol{c}, \boldsymbol{d}\})$ if a down move occurs. The payoff from waiting is therefore

$$
R(i, n,\{a, b, c, d\})+\frac{\pi_{u}(i, n) F(i, n+1,\{a, b, c, d\})+\pi_{d}(i, n) F(i+1, n+1,\{a, b, c, d\})}{R_{f}}(5) .
$$

In contrast, if Chorus chooses to construct, it is in one of four categories below at date $\boldsymbol{n}$, depending on the values of $\boldsymbol{a}, \boldsymbol{b}, \boldsymbol{c}$ and $\boldsymbol{d}$.

State category $D$ (when the state indicator $\{a, b, c, d\}$ equals $\{0,1,1,1\},\{1,1,0,1\},\{1,0,1,1\}$ or $\{1,1,1,0\})$ :

For example, if Chorus is in the state $\{\mathbf{0}, \mathbf{1}, \mathbf{1}, \mathbf{1}\}$, there is only one remaining city for it to construct at date $n$ (which is city HL), hence it pays $\operatorname{Cost}(\mathbf{1}, \mathbf{0}, \mathbf{0}, \mathbf{0})$ and immediately receives $\boldsymbol{R}(\boldsymbol{i}, \boldsymbol{n},\{\mathbf{0}, \mathbf{1}, \mathbf{1}, \mathbf{1}\})$, where

$$
\begin{gathered}
\operatorname{Cost}(1,0,0,0)=C \\
\text { and } R(i, n,\{0,1,1,1\})=X(i, n) \times(D R+2),
\end{gathered}
$$

At date $n+1$, the UFB contract will be worth $\boldsymbol{F}(\boldsymbol{i}, \boldsymbol{n}+\mathbf{1},\{\mathbf{1}, \mathbf{1}, \mathbf{1}, \mathbf{1}\})$ if an up move occurs and $\boldsymbol{F}(\boldsymbol{i}+\mathbf{1}, \boldsymbol{n}+\mathbf{1},\{\mathbf{1}, \mathbf{1}, \mathbf{1}, \mathbf{1}\})$ if a down move occurs. The payoff to Chorus from constructing in state $\{\mathbf{0}, \mathbf{1}, \mathbf{1}, \mathbf{1}\}$ is therefore

\footnotetext{
${ }^{41}$ For more detail see chapter 4, Guthrie (2009).
} 


$$
\begin{aligned}
R(i, n,\{0,1,1,1\}) & -\operatorname{Cost}(1,0,0,0) \\
& +\frac{\pi_{u}(i, n) F(i, n+1,\{1,1,1,1\})+\pi_{d}(i, n) F(i+1, n+1,\{1,1,1,1\})}{R_{f}} .
\end{aligned}
$$

From equation 5, we learn that the payoff to Chorus from waiting in state $\{\mathbf{0}, \mathbf{1}, \mathbf{1}, \mathbf{1}\}$ should be

$$
R(i, n,\{0,1,1,1\})+\frac{\pi_{u}(i, n) F(i, n+1,\{0,1,1,1\})+\pi_{d}(i, n) F(i+1, n+1,\{0,1,1,1\})}{R_{f}} .
$$

Since Chorus must choose between constructing city HL and waiting when it is in this state, it should adopt whichever action has the higher payoff, so that the present value of the UFB contract for Chorus at node $(\boldsymbol{i}, \boldsymbol{n})$ equals

$F(i, n,\{0,1,1,1\})$

$=\max \left\{\begin{array}{c}R(i, n,\{0,1,1,1\})+\frac{\pi_{u}(i, n) F(i, n+1,\{0,1,1,1\})+\pi_{d}(i, n) F(i+1, n+1,\{0,1,1,1\})}{R_{f}}, \\ R(i, n,\{0,1,1,1\})-\operatorname{Cost}(1,0,0,0)+\frac{\pi_{u}(i, n) F(i, n+1,\{1,1,1,1\})+\pi_{d}(i, n) F(i+1, n+1,\{1,1,1,1\})}{R_{f}}\end{array}\right\}(6)$.

The evaluations for all other states in category $\mathbf{D}$ follow the same process as state $\{\mathbf{0}, \mathbf{1}, \mathbf{1}, \mathbf{1}\}$.

State category $C$ (when the state indicator $\{a, b, c, d\}$ equals $\{0,0,1,1\},\{0,1,0,1\},\{0,1,1,0\}$, $\{1,0,0,1\},\{1,0,0,1\}$ or $\{1,1,0,0\})$ :

Similarly, we use one of the specified states to demonstrate the evaluation process in this category. For example, if Chorus is in the state $\{\mathbf{0}, \mathbf{0}, \mathbf{1}, \mathbf{1}\}$, there are two remaining cities for the firm to construct at date $n$, hence it can choose from the following three actions-pay $\operatorname{Cost}(1,0,0,0)$ to build up city HL, pay $\operatorname{Cost}(\mathbf{0}, 1,0,0)$ to build up city HH or pay $\operatorname{Cost}(\mathbf{1}, \mathbf{1}, \mathbf{0}, \mathbf{0})$ to build up two city together. No matter which decision Chorus makes it immediately receives $\boldsymbol{R}(\boldsymbol{i}, \boldsymbol{n},\{\mathbf{0}, \mathbf{0}, \mathbf{1}, \mathbf{1}\})$, where

$$
\begin{gathered}
\operatorname{Cost}(1,0,0,0)=C, \operatorname{Cost}(0,1,0,0)=C R \times C, \operatorname{Cost}(1,1,0,0)=(C R+1)^{2} C \\
\text { and } R(i, n,\{0,0,1,1\})=X(i, n) \times 2 .
\end{gathered}
$$

If Chorus chooses to construct city HL only, at date $\boldsymbol{n}+\mathbf{1}$, the UFB contract will be worth $\boldsymbol{F}(\boldsymbol{i}, \boldsymbol{n}+\mathbf{1},\{\mathbf{1}, \mathbf{0}, \mathbf{1}, \mathbf{1}\})$ if an up move occurs and $\boldsymbol{F}(\boldsymbol{i}+\mathbf{1}, \boldsymbol{n}+\mathbf{1},\{\mathbf{1}, \mathbf{0}, \mathbf{1}, \mathbf{1}\})$ if a down move occurs. The payoff to Chorus from constructing city $\mathrm{HL}$ is therefore

$$
\begin{aligned}
R(i, n,\{0,0,1,1\}) & -\operatorname{Cost}(1,0,0,0) \\
& +\frac{\pi_{u}(i, n) F(i, n+1,\{1,0,1,1\})+\pi_{d}(i, n) F(i+1, n+1,\{1,0,1,1\})}{R_{f}} .
\end{aligned}
$$


However, if Chorus chooses to construct city HH only, at date $\boldsymbol{n}+\mathbf{1}$, the UFB contract will be worth $\boldsymbol{F}(\boldsymbol{i}, \boldsymbol{n}+\mathbf{1},\{\mathbf{0}, \mathbf{1}, \mathbf{1}, \mathbf{1}\})$ if an up move occurs and $\boldsymbol{F}(\boldsymbol{i}+\mathbf{1}, \boldsymbol{n}+\mathbf{1},\{\mathbf{0}, \mathbf{1}, \mathbf{1}, \mathbf{1}\})$ if a down move occurs. The payoff to Chorus from constructing city $\mathrm{HH}$ is therefore

$$
\begin{aligned}
R(i, n,\{0,0,1,1\}) & -\operatorname{Cost}(0,1,0,0) \\
+ & \frac{\pi_{u}(i, n) F(i, n+1,\{0,1,1,1\})+\pi_{d}(i, n) F(i+1, n+1,\{0,1,1,1\})}{R_{f}} .
\end{aligned}
$$

Besides, if Chorus chooses to construct both cities together, at date $\boldsymbol{n}+\mathbf{1}$, the UFB contract will be worth $\boldsymbol{F}(\boldsymbol{i}, \boldsymbol{n}+\mathbf{1},\{\mathbf{1}, \mathbf{1}, \mathbf{1}, \mathbf{1}\})$ if an up move occurs and $\boldsymbol{F}(\boldsymbol{i}+\mathbf{1}, \boldsymbol{n}+\mathbf{1},\{\mathbf{1}, \mathbf{1}, \mathbf{1}, \mathbf{1}\})$ if a down move occurs. The payoff to Chorus from constructing city HL is therefore

$$
\begin{aligned}
R(i, n,\{0,0,1,1\}) & -\operatorname{Cost}(1,1,0,0) \\
+ & \frac{\pi_{u}(i, n) F(i, n+1,\{1,1,1,1\})+\pi_{d}(i, n) F(i+1, n+1,\{1,1,1,1\})}{R_{f}}
\end{aligned}
$$

From equation 5, we learn that the payoff to Chorus from waiting in state $\{\mathbf{0}, \mathbf{0}, \mathbf{1}, \mathbf{1}\}$ should be

$$
R(i, n,\{0,0,1,1\})+\frac{\pi_{u}(i, n) F(i, n+1,\{0,0,1,1\})+\pi_{d}(i, n) F(i+1, n+1,\{0,0,1,1\})}{R_{f}}
$$

Since the firm must choose from constructing city HL, constructing city $\mathrm{HH}$, constructing both cities and waiting, it should adopt whichever action has the higher payoff, so that the present value of the UFB contract for Chorus at node $(\boldsymbol{i}, \boldsymbol{n})$ equals

$F(i, n,\{0,0,1,1\})$

$$
=\max \left\{\begin{array}{c}
R(i, n,\{0,0,1,1\})+\frac{\pi_{u}(i, n) F(i, n+1,\{0,0,1,1\})+\pi_{d}(i, n) F(i+1, n+1,\{0,0,1,1\})}{R_{f}}, \\
R(i, n,\{0,0,1,1\})-\operatorname{Cost}(1,0,0,0)+\frac{\pi_{u}(i, n) F(i, n+1,\{1,0,1,1\})+\pi_{d}(i, n) F(i+1, n+1,\{1,0,1,1\})}{R_{f}} \\
R(i, n,\{0,0,1,1\})-\operatorname{Cost}(0,1,0,0)+\frac{\pi_{u}(i, n) F(i, n+1,\{0,1,1,1\})+\pi_{d}(i, n) F(i+1, n+1,\{0,1,1,1\})}{R_{f}} \\
R(i, n,\{0,0,1,1\})-\operatorname{Cost}(1,1,0,0)+\frac{\pi_{u}(i, n) F(i, n+1,\{1,1,1,1\})+\pi_{d}(i, n) F(i+1, n+1,\{1,1,1,1\})}{R_{f}}
\end{array}\right\}
$$

The evaluations for all other states in category $\mathbf{C}$ follow the same process as state $\{\mathbf{0}, \mathbf{0}, \mathbf{1}, \mathbf{1}\}$.

State category $B$ (when the state indicator $\{a, b, c, d\}$ equals $\{0,0,0,1\},\{0,0,1,0\},\{0,1,0,0\}$ or $\{1,0,0,0\})$ :

The payoffs of each construction action could be created in a same way as above; therefore we do not list them all here. Since the firm must choose from seven constructing actions and 
one waiting action, it should adopt whichever action has the higher payoff, so that the present value of the UFB contract for Chorus at node $(\boldsymbol{i}, \boldsymbol{n})$ equals

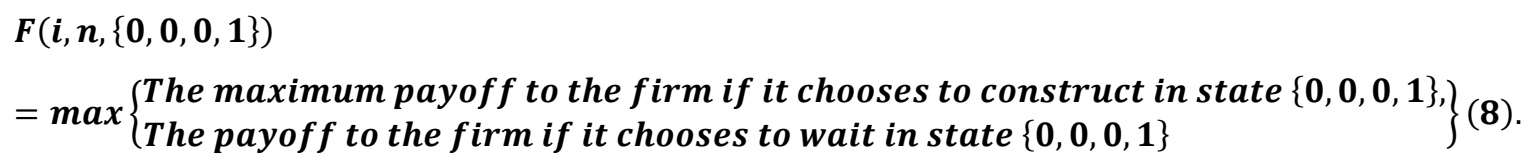

The evaluations for all other states in category $B$ follow the same process as state $\{\mathbf{0 , 0}, \mathbf{0}, \mathbf{1}\}$. State category $A$ (when the state indicator $\{a, b, c, d\}$ equals $\{0,0,0,0\})$ :

If the firm is in this category, it will face all sixteen choices. Rather than list them all here, we just note that they are similar to those in equation 7 , where

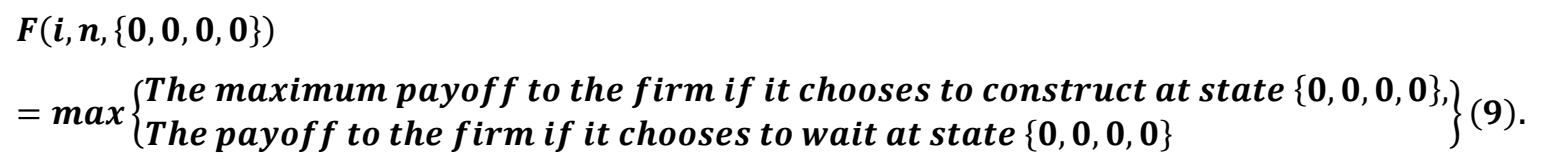

Actually, the general evaluation formula for all categories will be

$\boldsymbol{F}(\boldsymbol{i}, \boldsymbol{n},\{\boldsymbol{a}, \boldsymbol{b}, \boldsymbol{c}, \boldsymbol{d}\})$

$=\max \left\{\begin{array}{l}\text { The maximum payoff to the firm if it chooses to construct at state }\{a, b, c, d\}, \\ \text { The payoff to the firm if it chooses to wait at state }\{a, b, c, d\}\end{array}\right\}(10)$.

This equation, together with the terminal condition (equation 4), completely determines the present value of the UFB contract for Chorus at date 0 .

\subsubsection{Valuation process for CFH (CFH optimal policy)}

Let $\boldsymbol{T}(\boldsymbol{i}, \boldsymbol{n},\{\boldsymbol{a}, \boldsymbol{b}, \boldsymbol{c}, \boldsymbol{d}\})$ denote the present value of the UFB contract for CFH assuming that CFH chooses its own policy at the node $(\boldsymbol{i}, \boldsymbol{n})$ when the state of construction is $\{\boldsymbol{a}, \boldsymbol{b}, \boldsymbol{c}, \boldsymbol{d}\}$, where $\boldsymbol{a}, \boldsymbol{b}, \boldsymbol{c}$ and $\boldsymbol{d}$ equal the numbers of construction periods that have been finished in cities HL, HH, LH and LL cities respectively. Similar to our valuation of Chorus, the values of $\boldsymbol{a}, \boldsymbol{b}, \boldsymbol{c}$ and $\boldsymbol{d}$ here will be either 0 or 1 as well, where 1 means that the city has been finished and vice versa for 0 . The evaluation process to $\mathrm{CFH}$ is almost the same as the evaluation process for Chorus; therefore we are not going to repeatedly list it here. The only difference we make to the valuation process for $\mathrm{CFH}$ is that we include consumer surplus in CFH's payoffs. For example, if the state of construction is $\{\mathbf{0}, \mathbf{1}, \mathbf{1}, \mathbf{1}\}$ at date $n$, the flow of surplus that CFH receives should have $R(i, n,\{0,1,1,1\})$ equalled to $\left[(D R+2) \times X(i, n)+\left(D R^{2}+2\right) \times\right.$ $V(i, n)]$ rather than $X(i, n)(D R+2)$. 


\subsubsection{Valuation process for CFH (Chorus optimal policy)}

Since we want to compare the overall welfare when Chorus makes the investment decisions with the overall welfare when $\mathrm{CFH}$ makes the investment decisions (which we have done at section 4.5.2), we mix CFH's objective function with Chorus' optimal decisions in this section. Let $\boldsymbol{G}(\boldsymbol{i}, \boldsymbol{n},\{\boldsymbol{a}, \boldsymbol{b}, \boldsymbol{c}, \boldsymbol{d}\})$ denote the present value of the UFB contract to CFH under Chorus' optimal policy at the node $(\boldsymbol{i}, \boldsymbol{n})$ when the state of construction is $\{\boldsymbol{a}, \boldsymbol{b}, \boldsymbol{c}, \boldsymbol{d}\}$, where $\boldsymbol{a}, \boldsymbol{b}, \boldsymbol{c}$ and $\boldsymbol{d}$ have the same meaning as in the previous section. The valuation process will be quite similar to that in section 4.5.2 (under CFH's optimal policy). However, the maximization process among different policies (equation 8) could be ignored here since the CFH will follow exactly the same policy as Chorus. Therefore, we just need to learn Chorus' specified policy at each node. For example, if waiting is the adopted policy at node $(\mathbf{0}, \mathbf{3})$ in state $\{\mathbf{0}, \mathbf{0}, \mathbf{0}, \mathbf{0}\}$ and then we can get the present value at that point $\boldsymbol{G}(\mathbf{0}, \mathbf{3},\{\mathbf{0}, \mathbf{0}, \mathbf{0}, \mathbf{0}\})$ from estimating the payoff function corresponding to that specified policy (the payoff to $\mathrm{CFH}$ at node $(\mathbf{0}, \mathbf{3})$ if it chooses to wait in next period).

\subsection{Results for basic model}

\subsubsection{Conflicts between Chorus and CFH}

In our model, there are two sorts of conflicts between Chorus and CFH. A) The conflict of construction timing, in which Chorus and $\mathrm{CFH}$ may have different opinions about choosing when to construct the fibre network (parties disagree on whether construction is optimal in the next period). B) The conflict of construction sequence, in which the two parties may have different ideas about choosing the construction order among different cities (they consider construction in the next period is optimal but disagree about which city should go next). We would like to apply our numerical example to demonstrate these two conflicts. All values of parameters for our numerical example can be found at table 4.1. Any modification will be notified.

We provide the most interesting information about the optimal construction policies for both Chorus and $\mathrm{CFH}$ at table 4.2 and table 4.3 respectively. We only report the information about the first six periods due to the limited space (there are twelve periods in total). Specifically, these tables describe the optimal policies once city HL has been finished. Both parties are deciding when, and what, to build next. Every cell at table 4.2 or table 4.3 represents the adopted construction behaviour of the underlying party corresponding to the 
node $(\boldsymbol{i}, \boldsymbol{n})$. For example, the first entry at table 4.2 is $\{\mathbf{0 , 0 , 0}, \mathbf{0}\}$, which means that Chorus would like to wait at node $(\mathbf{0}, \mathbf{0})$.

Table 4.2: Chorus's optimal policy at state $(1,0,0,0)$

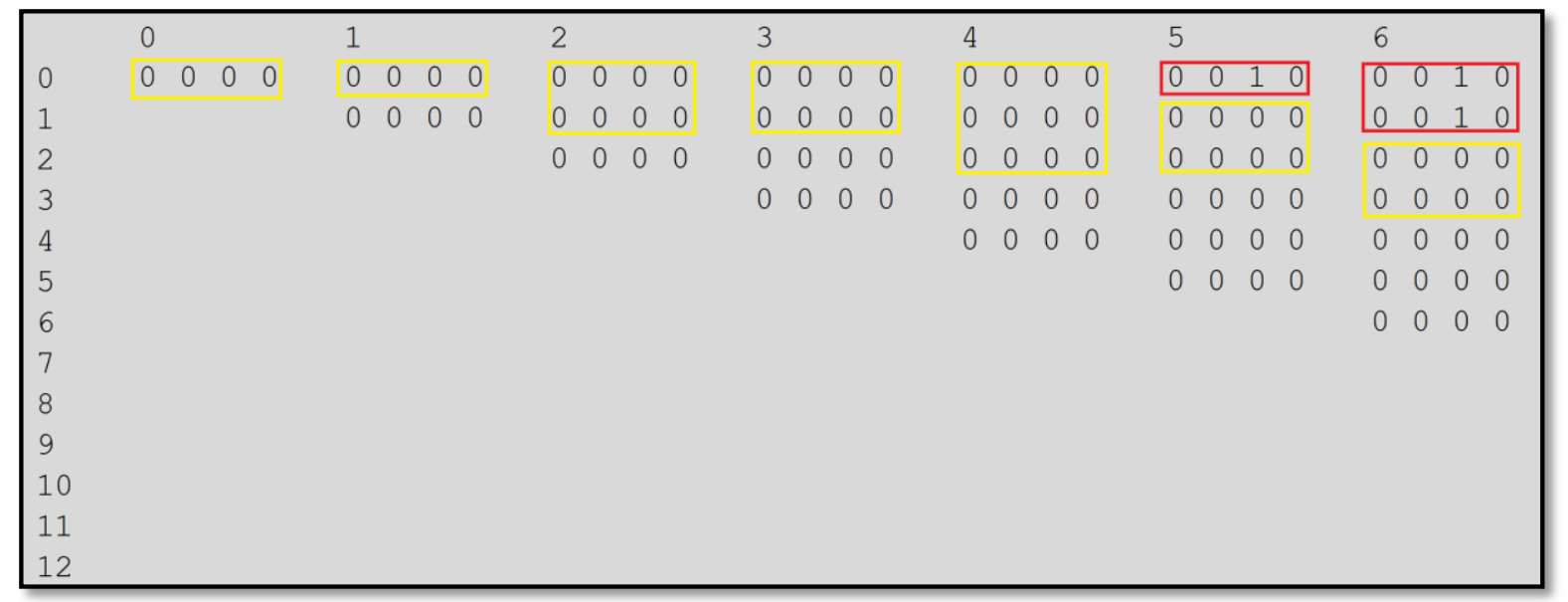

Table 4.3: CFH's optimal policy at state $(1,0,0,0)$

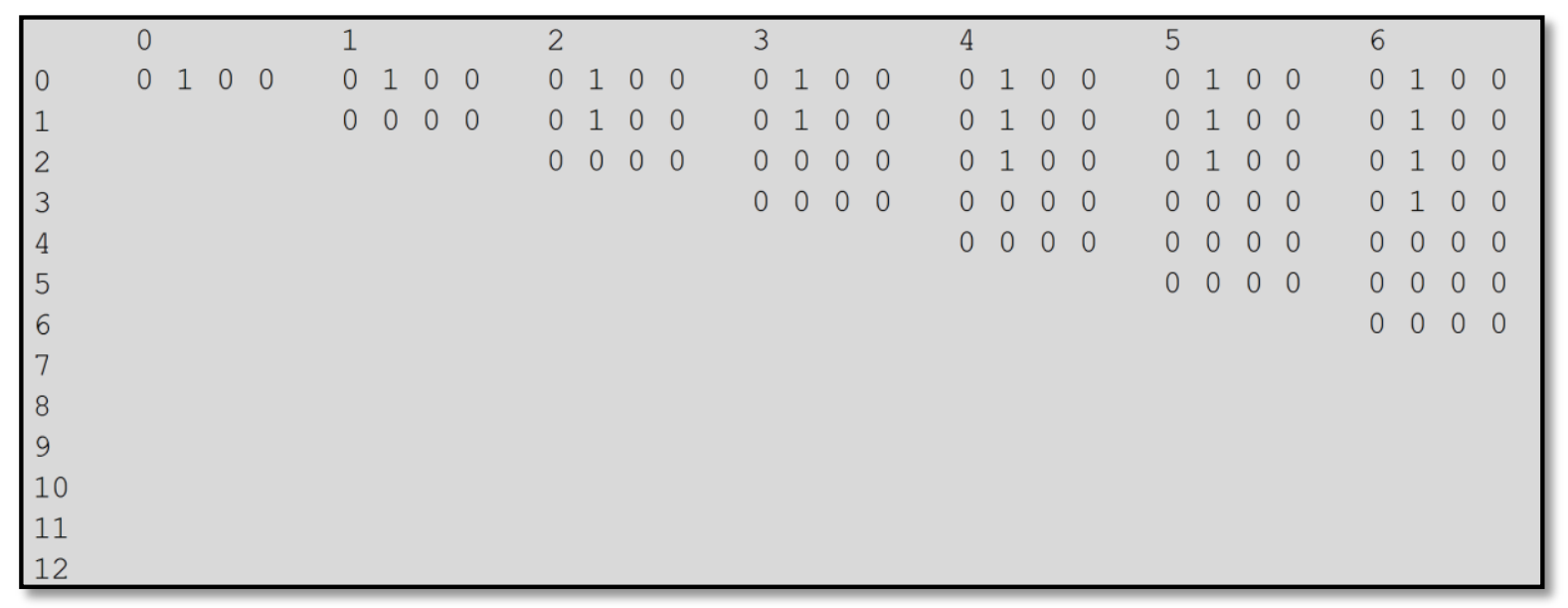

\section{A) The conflict of construction timing}

The conflicts of construction timing could be identified from comparing table 4.2 and table 4.3, which are highlighted by yellow boxes. For example, if CFH and Chorus are in state (1, $0,0,0)$ during date 0 to date 2 , there is a timing conflict between two parties if the current state is not too bad. (That is, not all the past moves are down moves) At date 3, the timing conflict only disappears if at least 2 down moves have occurred in the past. However, at date 4, the threshold for the timing conflict to be able to disappear has extended to at least 3 down moves having occurred in the past. At date 5, the timing conflict does not show up when 
there have been 5 up moves or there have been at least 3 down moves. At date 6 , the timing conflict vanishes when there have been 5 up moves or there have been at least 4 down moves.

\section{B) The conflict of construction sequence}

In contrast, we highlight the sequencing conflicts by red boxes. As we may find in table 4.2 and table 4.3 , the sequencing conflict only exists when the current level of demand is high. ${ }^{42}$ For example, at date 5 and date 6 , the sequencing conflict only shows up when there have been more than four up moves.

\subsubsection{The consequences of conflict}

If $\mathrm{CFH}$ works in the best interests of society as a whole, its optimal policy will maximise social welfare. Therefore, any policy which deviates from CFH's optimal policy may bring a potential social welfare loss (the present value of conflict), where these welfare losses may be raised either from an undersupply in the UFB network during certain periods ${ }^{43}$ or an incorrect priority policy among different cities. ${ }^{44}$ And it will be useful to measure the potential social welfare losses from the adopted suboptimal policies. In our numerical example, we apply equation 11 and equation 12 to calculate the present value of social welfare loss and the proportion of conflict respectively.

$$
\begin{aligned}
& \text { Present value of welfare loss }=T(0,0,\{a, b, c, d\})-G(0,0,\{a, b, c, d\})(11) \\
& \text { The proportion of conflict }=1-\frac{G(0,0,\{a, b, c, d\})}{T(0,0,\{a, b, c, d\})}
\end{aligned}
$$

Where $\boldsymbol{T}(\mathbf{0}, \mathbf{0},\{\boldsymbol{a}, \boldsymbol{b}, \boldsymbol{c}, \boldsymbol{d}\})$ is the present value of the UFB contract for CFH at state $\{\boldsymbol{a}, \boldsymbol{b}, \boldsymbol{c}, \boldsymbol{d}\}$ under CFH's optimal policy (therefore in the view of society, it is the maximised present value of social welfare raised from the UFB project), and $\boldsymbol{G}(\mathbf{0}, \mathbf{0},\{\boldsymbol{a}, \boldsymbol{b}, \boldsymbol{c}, \boldsymbol{d}\})$ is the present value of the UFB contract for CFH at state $\{\boldsymbol{a}, \boldsymbol{b}, \boldsymbol{c}, \boldsymbol{d}\}$ under Chorus's optimal policy. (Therefore in the view of society, it is only one of the social welfare present values rather than the maximum.)This present value may be equal to or smaller than the maximum depending on whether Chorus's optimal policy is the same as CFH's. If state $\{\boldsymbol{a}, \boldsymbol{b}, \boldsymbol{c}, \boldsymbol{d}\}$ is

\footnotetext{
${ }^{42}$ It does not mean high demand always bring sequencing conflicts. For example, at date 5 , when there have been five up moves, a timing conflict rather than a sequencing conflict shows up since the demand is high enough to induce CFH to build up both city $\mathrm{HH}$ and city LL once.

${ }^{43}$ The firm may work too slowly to provide enough supply to meet the social demand.

${ }^{44}$ For saving the cost, the firm may give priority to the low cost city instead of the high cost city, even though the high cost city has a higher total surplus than that of the low cost city.
} 
equal to $\{\mathbf{0}, \mathbf{0}, \mathbf{0}, \mathbf{0}\}$, we have Table 4.4 for $\boldsymbol{T}(\boldsymbol{i}, \boldsymbol{n},\{\mathbf{0}, \mathbf{0}, \mathbf{0}, \mathbf{0}\})$ and Table 4.5 for $\boldsymbol{G}(\boldsymbol{i}, \boldsymbol{n},\{\mathbf{0}, \mathbf{0}, \mathbf{0}, \mathbf{0}\})$ respectively.

Table 4.4: Present value of UFB for CFH under Chorus policy at state $(0,0,0,0)$

\begin{tabular}{|llllllll|}
\hline & 0 & 1 & 2 & 3 & 4 & 5 & 6 \\
0 & 76.8053 & 99.6568 & 130.807 & 173.501 & 231.682 & 309.151 & 408.719 \\
1 & & 58.4456 & 74.812 & 96.9915 & 127.712 & 170.815 & 229.753 \\
2 & & & 45.1625 & 56.95 & 72.4467 & 93.5352 & 123.28 \\
3 & & & & 35.4927 & 44.3253 & 55.4041 & 69.7049 \\
4 & & & & & 28.1812 & 35.1971 & 43.6916 \\
5 & & & & & & & \\
6 & & & & & & & \\
7 & & & & & & & \\
8 & & & & & & & \\
9 & & & & & & & \\
10 & & & & & & & \\
11 & & & & & & & \\
12 & & & & & & & \\
\hline
\end{tabular}

Table 4.5: Present value of UFB for Chorus under Chorus policy at state $(0,0,0,0)$

\begin{tabular}{|llllllll|}
\hline & 0 & 1 & 2 & 3 & 4 & 5 & 6 \\
0 & 105.823 & 136.745 & 175.025 & 221.663 & 278.545 & 348.064 & 433.32 \\
1 & & 80.8534 & 105.567 & 136.648 & 174.887 & 221.454 & 278.177 \\
2 & & & 61.0785 & 80.4707 & 105.29 & 136.563 & 174.779 \\
3 & & & & 45.6891 & 60.4587 & 80.04 & 105.048 \\
4 & & & & & 33.8825 & 44.9135 & 59.7518 \\
5 & & & & & & & \\
6 & & & & & & & \\
7 & & & & & & & \\
8 & & & & & & & \\
9 & & & & & & & \\
10 & & & & & & & \\
11 & & & & & & & \\
12 & & & & & & & \\
\hline
\end{tabular}

The present values of social welfare under two different policies have been highlighted by yellow boxes. With equation 11 and 12, we find the present value of welfare loss and proportion of conflict equal 29 and $\mathbf{0 . 2 7 4}$ respectively. 


\section{Chapter 5: The general model setting-up}

\subsection{A shift from the basic model to the general model}

In our basic model, we assume that the construction of a fibre network in any single city can be completed in one period. This implies that the construction process cannot be interrupted or sped up, so that both parties have no flexibility after launching the fibre roll-out in individual cities. Although this assumption dramatically simplifies our model, it may be too simple to be realistic since the firm may actually have some flexibility during the construction period, especially for such a complicated project ${ }^{45}$. Besides, allowing more periods to build up one city implies that there will be a smaller $\boldsymbol{d} t$ to make our model more accurate. Last but not least, since more flexibility has been allocated to the private party, the general model give us more levels of contract incompleteness to study. Actually, with the single period model, the lump sum subsidy and fixed period subsidy will have the same effects in motivating the private firm to follow the public party's optimal policy since suspension and speed-up options are unavailable. Therefore, we should consider a more general case, in which every city requires more than one period to finish.

In order to allow multiple-period construction, we keep $\boldsymbol{T}$ (UFB contract duration) unchanged but reduce the time measure for each step $\boldsymbol{d} t$. Although a smaller $\boldsymbol{d} t$ will result in a more accurate result, the increase of the number of periods to build exponentially increases the time for calculation during programing. Therefore, to have a more accurate estimation but avoid the explosion of calculations, we only reduce $\boldsymbol{d t}$ from 0.5 to 0.25 in our general model. That is, we cap the number of periods to build up one city at two, which suggests that the firm maximally spend two periods to finish the fibre network construction in each city. Apart from this, several parameters for the general model are changed from the basic model. We report the values of all parameters for the general model in Table 5.1.

\footnotetext{
${ }^{45}$ Since flexibility is always valuable, it is costly for $\mathrm{CFH}$ to take all flexibilities away from Chorus during the fibre network roll-out. Besides, it may not be in CFH's best interest to exclude both the suspension option and the speed-up option during fibre construction because they may want to exercise these options in certain states as well. The complexity of the project may make it technologically infeasible for CFH to exclude the company's suspension and speed-up options during the construction period. This is because, in order to exclude any potential suspension, CFH needs to have perfect information about the states of all cities (which city is $\mathrm{HL}$, which city is $\mathrm{HH}$, etc.), which is the firm's private information.
} 
Table 5.1: Parameter values for the two-period model

\begin{tabular}{|c|c|c|}
\hline Parameter & Symbol & Value \\
\hline \multicolumn{3}{|l|}{ a) Real world parameter } \\
\hline Annual demand Volatility & $\sigma$ & 0.15 \\
\hline Present value for $\boldsymbol{X}_{\mathbf{0}} *$ & $P V P S$ & 8.5 \\
\hline Present value for $\boldsymbol{C} \boldsymbol{S}_{\mathbf{0}} *$ & PVCS & 2.87 \\
\hline Total capital expenditure $*$ & C & 10 \\
\hline Expected growth rate & $\boldsymbol{\mu}$ & 0.07 \\
\hline Annual risk-free interest rate & $r_{f}$ & 0.015 \\
\hline UFB contract duration (measured by year) & $\boldsymbol{T}$ & 6 \\
\hline UFB contract period & $N$ & 24 \\
\hline Cost ratio & CR & 2 \\
\hline Demand ratio & $D R$ & 3 \\
\hline $\begin{array}{l}\text { the constant multiplier between consumer and } \\
\text { producer surpluses }\end{array}$ & Con & 4 \\
\hline \multicolumn{3}{|l|}{ b) Model parameter } \\
\hline Time for each period & $d t$ & 0.25 \\
\hline Time to build up one city (measured by year) & $\boldsymbol{Y}$ & 0.5 \\
\hline Per-period capital expenditure & & 5 \\
\hline Per-period risk adjusted growth factor & $K$ & 0.997 \\
\hline Per-period growth factor (up) & $\boldsymbol{U}_{\mathbf{0}}$ & 1.078 \\
\hline Per-period growth factor (down) & $D_{0}$ & 0.928 \\
\hline Per-period risk-neutral probability (up) & $\pi_{u}$ & 0.456 \\
\hline Per-period risk-neutral probability (down) & $\pi_{d}$ & 0.544 \\
\hline Current revenue* & $X_{0}$ & 0.064 \\
\hline Current consumer surplus* & $C S_{0}$ & 0.016 \\
\hline Per-period risk-free interest factor & $\boldsymbol{R}_{\boldsymbol{f}}$ & 1.004 \\
\hline
\end{tabular}

\subsection{Decision table for two-period model}

In practice, it is very difficult to draw the decision tree for multiple-period models due to the large number of actions available to the decision maker. In the case of the two-period model (the simplest multiple-period case), the players can choose from the following three kinds of option for each city that has not yet started its fibre roll out - finishing the first stage of construction for this city, finishing the whole construction for this city or waiting.

Combining the four types of cities we have, there are $3^{4}=81$ states in a two-period model 
decision tree, which is very messy (the reader may compare it with the decision tree of our basic model (which has only 16 states) to imagine how messy the decision tree will be). To illustrate the possibilities, we illustrate the actions available once the constructions of two cities have been completed, meaning that the firm only needs to consider two remaining cities when it is making the decisions (There are only city HH (high demand city with high cost) and city LH (low demand city with high cost) left, which dramatically reduce the number of states from 81 to 9$).{ }^{46}$ Since the reduction of city number only exists to demonstrate the firm's decision marking, it does not affect the valuation and results of the general model. ${ }^{47}$ We summarize players' decisions in table 5.2.

Table 5.2: Decision table for two-period two cities model

\begin{tabular}{|l|l|l|l|l|l|l|l|l|l|}
\hline & $\{0,0\}$ & $\{0,1\}$ & $\{0,2\}$ & $\{1,0\}$ & $\{2,0\}$ & $\{1,1\}$ & $\{1,2\}$ & $\{2,1\}$ & $\{2,2\}$ \\
\hline$\{0,0\}$ & $\checkmark$ & $\checkmark$ & $\checkmark$ & $\checkmark$ & $\checkmark$ & $\checkmark$ & $\checkmark$ & $\checkmark$ & $\checkmark$ \\
\hline$\{0,1\}$ & & $\checkmark$ & $\checkmark$ & & & $\checkmark$ & $\checkmark$ & $\checkmark$ & $\checkmark$ \\
\hline$\{0,2\}$ & & & $\checkmark$ & & & & $\checkmark$ & & $\checkmark$ \\
\hline$\{1,0\}$ & & & & $\checkmark$ & $\checkmark$ & $\checkmark$ & $\checkmark$ & $\checkmark$ & $\checkmark$ \\
\hline$\{2,0\}$ & & & & & $\checkmark$ & & & $\checkmark$ & $\checkmark$ \\
\hline$\{1,1\}$ & & & & & & $\checkmark$ & $\checkmark$ & $\checkmark$ & $\checkmark$ \\
\hline$\{1,2\}$ & & & & & & & $\checkmark$ & & $\checkmark$ \\
\hline$\{2,1\}$ & & & & & & & & $\checkmark$ & $\checkmark$ \\
\hline$\{2,2\}$ & & & & & & & & & \\
\hline
\end{tabular}

In Table 5.2, each column represents the possible future states in the next period, while each row represents the possible current states. The first number in the bracket indicates the stage of construction for city $\mathrm{HH}$, while the second number indicates the stage of construction for city LH. For example, $\{0,0\}$ in a row indicates that the player has started neither city at the current stage; $\{0,0\}$ in a column indicates that neither city will be started even in the next period. Ticks have been used to indicate the possible transitions between current states (row states) to corresponding future states (column states). Consider the following example. If the

\footnotetext{
${ }^{46}$ Since there are three choices that can be made for each city and two types of city left, the number of total states equals $3^{2}=9$.

${ }^{47}$ Although we only provide the decision tree for the two-period two cities case since it is complicated enough, the decision tree for any more complicated models (Such as two-period four cities and three-period four cities, etc.) could be derived by the same method.
} 
player is in the first row at date 0 (which is $\{0,0\}$ ), he has not started any of these two cities, therefore one action he may take is waiting, which is the first tick; Or he may start to build up one of these two cities but not go full speed, which corresponds to the second tick if he is doing city LH or the fourth tick if he is doing city HH; Or he may start to build up one of these two cities with the full speed, which corresponds to the third tick if he is doing city LH or the fifth tick if he is doing city HH; Or he may start to build up both cities, which corresponds to the last four ticks (depending on different levels of speed). All above possible behaviours are motivated by the player's economics consideration. That is, by waiting, the player has a chance to observer whether the demand goes up or down; by investing in two stages at once (full speed), although the player incurs the higher capital expenditure, he can earn the revenues earlier. The player chooses its optimal behaviour according to the trade-off between capital expenditures and revenues. All his future states are determined by which transitions are taken from date 0 to date 1 .

Now assume the player already took the action $\{0,1\}$ at a previous period, hence he is in row 2 now. Once again, he can choose to wait to see whether demand goes up or down, which corresponds to the suspension option (the first tick in this column); or he can earn the revenues earlier by finishing city $\mathrm{LH}$, which corresponds to the second tick in this row; or he may start city $\mathrm{HH}$, which corresponds to the third tick if he only starts the first stage or the fifth tick if he is trying to finish both stages (which the player is exercising a speed-up option); or he may finish city LH and start city HH simultaneously, which corresponds to the fourth tick if he just starts the first stage of city $\mathrm{HH}$ or the sixth tick if he is working through both stages of city $\mathrm{HH}$ (once again, the player is choosing to exercise a speed-up option).As above, the optimal behaviour of the player depends on the trade-off between its capital expenditures and revenues.

Similarly, we can derive all possible transactions in row three, row four..., and row nine. The process continues until either the player complete all constructions (that is the ninth row $\{2,2\})$ or the development right expires. At these points no more decisions need to be made by the firm and all future cash flows can be estimated without the decision table.

\subsection{The valuation process for the general model}

In this section, the valuation processes for the general model are introduced. Similar to that of the basic model, there are three valuation processes for the general model. That is the valuation process of the general model for Chorus (Chorus's optimal policy), valuation 
process of the general model for CFH (CFH's optimal policy) and valuation process of the general model for CFH (Chorus's optimal policy). In practice, all three valuation processes have a few changes when we shift from the basic model to the general model. However, we only describe the detail of the valuation process for Chorus as we need these evaluation equations to introduce the add-on components of incentive tools in the next section.

\subsubsection{Valuation process of the general model for Chorus (Chorus optimal policy)}

In the general model, we still use $\boldsymbol{F}(\boldsymbol{i}, \boldsymbol{n},\{\boldsymbol{a}, \boldsymbol{b}, \boldsymbol{c}, \boldsymbol{d}\})$ to denote the present value of the UFB contract for Chorus at the node $(i, n)$ when the firm is in the state $\{a, b, c, d\}$. However, in contrast to the basic case, the values of a,b,c and d will be 0,1 or 2 , where 2 means that both stages of this city have been finished, 1 means that only one stage of this city has been finished, while 0 means that none of the stages of this city has been done.

As in the basic model, the terminal condition for the general model should equal either $\frac{\boldsymbol{R}_{f} \boldsymbol{R}(\boldsymbol{i}, \boldsymbol{N},\{\boldsymbol{a}, \boldsymbol{b}, \boldsymbol{c}, \boldsymbol{d}\})}{\boldsymbol{R}_{\boldsymbol{f}}-\boldsymbol{K}}$ or $\frac{\boldsymbol{R}_{\boldsymbol{f}} \boldsymbol{R}(\boldsymbol{i}, \boldsymbol{n},\{\mathbf{2}, \mathbf{2 , 2 , 2 , 2 \} )}}{\boldsymbol{R}_{\boldsymbol{f}}-\boldsymbol{K}},{ }^{48}$ which is equation 4 .

At any non-terminal node ${ }^{49}$, the present value of the UFB contract for the private party at node $(\mathbf{i}, \mathbf{n})$ when the firm is in current state $\{a, b, c, d\}$ equals,

$$
\begin{aligned}
& F(i, n,\{a, b, c, d\}) \\
& =\max \left\{R(i, n,\{a, b, c, d\})-\operatorname{Capex}\left(j_{a}, j_{b}, j_{c}, j_{d}\right)+S\right. \\
& \left.+\frac{\pi_{u}(i, n) F\left(i, n+1,\left\{a+j_{a}, b+j_{b}, c+j_{c}, d+j_{d}\right\}\right)+\pi_{d}(i, n) F\left(i+1, n+1,\left\{a+j_{a}, b+j_{b}, c+j_{c}, d+j_{d}\right\}\right)}{R_{f}}\right\}
\end{aligned}
$$

where $j_{x} \in(0$, periodstobuild $-x), x=a, b, c$ or d

for city $H L$, city $H H$, cityLL and city $L H$ respectively (11).

In this equation, $S$ is the cash flow generated by the subsidy scheme, which we are going to introduce in the next section. $\boldsymbol{R}(\boldsymbol{i}, \boldsymbol{n},\{\boldsymbol{a}, \boldsymbol{b}, \boldsymbol{c}, \boldsymbol{d}\})$ is the revenue the private party can receive during this period. $\operatorname{Capex}\left(\boldsymbol{j}_{\boldsymbol{a}}, \boldsymbol{j}_{\boldsymbol{b}}, \boldsymbol{j}_{\boldsymbol{c}}, \boldsymbol{j}_{\boldsymbol{d}}\right)$ is the capital expenditure for the private party in this period. $\boldsymbol{j}_{\boldsymbol{x}}$ indicates the number of construction stage undertaken for each city respectively during this period, with values ranging from 0 to periodstobuild $-\boldsymbol{x}$ (the number of stages

\footnotetext{
${ }^{48}$ In the case of both the simple fine scheme and two dimensional fine scheme introduced below, the terminal condition $\frac{\boldsymbol{R}_{f} \boldsymbol{R}(\boldsymbol{i}, \boldsymbol{N},\{\boldsymbol{a}, \boldsymbol{b}, \boldsymbol{c}, \boldsymbol{d}\})}{\boldsymbol{R}_{f}-\boldsymbol{K}}$ switches to $\frac{\boldsymbol{R}_{f} \boldsymbol{R}(\boldsymbol{i}, \boldsymbol{N},\{\boldsymbol{a}, \boldsymbol{b}, \boldsymbol{c}, \boldsymbol{d}\})}{\boldsymbol{R}_{f}-\boldsymbol{K}}-\boldsymbol{P}_{\mathbf{2 4}}$.

${ }^{49}$ At non-terminal condition, neither $\{\mathbf{a}, \mathbf{b}, \mathbf{c}, \mathbf{d}\} \neq\{2,2,2,2\}$ nor $\boldsymbol{n} \neq \boldsymbol{N}$.
} 
remaining to be completed for each city). For example, if each city takes two periods to be finished and the current state is $\{\boldsymbol{a}, \boldsymbol{b}, \boldsymbol{c}, \boldsymbol{d}\}=\{\mathbf{1}, \mathbf{1}, \mathbf{1}, \mathbf{1}\}$, the values of $\boldsymbol{j}_{\boldsymbol{x}}$ will range from 0 to 1 .

For any given current state $\{\boldsymbol{a}, \boldsymbol{b}, \boldsymbol{c}, \boldsymbol{d}\}$, the private party will choose a combination of $\boldsymbol{j}_{\boldsymbol{x}}$ (the optimal construction policy) that maximises the value of $\boldsymbol{F}(\boldsymbol{i}, \boldsymbol{n},\{\boldsymbol{a}, \boldsymbol{b}, \boldsymbol{c}, \boldsymbol{d}\})$. However, the public party can induce the private firm to change its combination of $\boldsymbol{j}_{\boldsymbol{x}}$ by setting various $\boldsymbol{S}$. Since $S$ is only the monetary transfer between the public and private party, it does not directly affect the public party's evaluation formulas. ${ }^{50}$ The public party will try to find out the optimal $\boldsymbol{S}$ to maximise the value of $\boldsymbol{G}(\mathbf{0}, \mathbf{0},\{\boldsymbol{a}, \boldsymbol{b}, \boldsymbol{c}, \boldsymbol{d}\})$, therefore minimizing the value of $\boldsymbol{T}(\mathbf{0}, \mathbf{0},\{\boldsymbol{a}, \boldsymbol{b}, \boldsymbol{c}, \boldsymbol{d}\})-\boldsymbol{G}(\mathbf{0}, \mathbf{0},\{\boldsymbol{a}, \boldsymbol{b}, \boldsymbol{c}, \boldsymbol{d}\})$, which is the present value of conflicts. ${ }^{51}$

\subsection{Optimal contracting}

In this section, we would like to introduce several incentive vehicles that help reduce the possible conflicts. The first sort of incentive vehicles we want to include will be the subsidy policies, which are the monetary transfers from one party to the other party in order to align their interests. Four levels of subsidy completeness have been studied in order to understand the effects of information asymmetry in the UFB case. Alternatively, two fine policies could be applied by the public party to discipline the private party in order to reduce their conflicts. Figuratively speaking, we may call the combination of subsidy and fine the carrot-and-stick approach, where the subsidy is the carrot and the fine is the stick. In the end, an optimal contract could be designed by combining all available incentive vehicles.

\subsubsection{An add-on of subsidy schemes for the general model}

\section{1) Proportional subsidy policy description}

If the monetary transfer could be proportional to the private party's capital expenditure level, we call it a proportional subsidy. However, in order to apply a proportional subsidy scheme, the public party needs to have enough inside information about the private party, which means that the public party needs to learn both the capital expenditure of each possible

\footnotetext{
${ }^{50}$ As we know, there are two valuation processes for the public party, one is under the private firm's optimal policy and the other is under the public party's own optimal policy. However, the valuation formulas are the same except different optimal policies have been applied during the maximization process. This is because the goal of the social planner (public party) is maximizing the total surplus, therefore a monetary transfer between parties does not change the social planner's utility function unless the tax distortion and agency cost for transfer are considered. However, we assume these two problems do not exist in our model. The tax distortion and agency cost on the subsidy in PPPs are discussed in Engle, Fischer and Galetovic (2007).

${ }^{51}$ Since $\boldsymbol{T}(\mathbf{0}, \mathbf{0},\{\boldsymbol{a}, \boldsymbol{b}, \boldsymbol{c}, \boldsymbol{d}\})$ is the first best condition, it will be constant for any given $\{\boldsymbol{a}, \boldsymbol{b}, \boldsymbol{c}, \boldsymbol{d}\}$.
} 
transition and construction behaviours from the private firm. ${ }^{52}$ The first part tells them the value of each component for total capital expenditure, while the second part gives them the information about their possible combinations in each period.

\section{2) Proportional subsidy component add-on in evaluation formula}

In order to include the proportional subsidy into our general model evaluation, a proportional subsidy component needs to be introduced. That is, $\boldsymbol{S}$ equals $\boldsymbol{P S} \times\left(\boldsymbol{C a p e x}\left(\boldsymbol{j}_{a^{\prime}}, \boldsymbol{j}_{\boldsymbol{b}}, \boldsymbol{j}_{c^{\prime}} \boldsymbol{j}_{d}\right)\right)$ in equation 11, where $\boldsymbol{P S}$ stands for the rate of proportional subsidy.

\section{3) Fixed period subsidy policy description}

If the public party is unable to observe the specified construction behaviour of the private firm, they cannot make the monetary transfer proportional to the private party's current capital expenditure level. ${ }^{53}$ Therefore, an efficient proportional subsidy is no longer available. In this case, we may need to look for a less complete contract, which is the fixed period subsidy policy. It does not require that the public party knows about the firm's actions specifically (Less information required). However, the public party still needs to learn how many construction stages have been finished by the private firm during the last period. This is because, although the level of fixed subsidy is irrelevant to the states of construction, it is proportional to the number of construction stage that has been finished. For example, if the state of construction $\left\{\boldsymbol{j}_{a}, \boldsymbol{j}_{\boldsymbol{b}}, \boldsymbol{j}_{c}, \boldsymbol{j}_{\boldsymbol{d}}\right\}=\{\mathbf{1}, \mathbf{1}, \mathbf{1}, \mathbf{1}\}$, a proportional subsidy policy may requires the public party to learn the values of all $\boldsymbol{j}_{\boldsymbol{x}}$, while a fixed subsidy policy only require the public party to know the sum of $\boldsymbol{j}_{\boldsymbol{x}}$ rather than its specific values. In other words, unlike the proportional subsidy scheme, the fixed subsidy scheme does not require that the player is able to distinguish high and low cost cities.

\section{4) Fixed period subsidy component add-on in evaluation formula}

Similar to the proportional subsidy policy, we include a fixed subsidy component in our general model evaluation here. That is, $\boldsymbol{S}$ equals $\boldsymbol{F} \boldsymbol{S} \times\left(\boldsymbol{j}_{\boldsymbol{a}}+\boldsymbol{j}_{\boldsymbol{b}}+\boldsymbol{j}_{\boldsymbol{c}}+\boldsymbol{j}_{\boldsymbol{d}}\right)$ in equation $12, \boldsymbol{F} \boldsymbol{S}$ is a fixed level of monetary transfer from the public party to the private party for each finished city during this period, and the total level of fixed subsidy depends on the sum of $\boldsymbol{j}_{\boldsymbol{x}}$.

\footnotetext{
52 The public party knows how much to construct each stage for each city. Besides, the firm cannot hide its behaviour from $\mathrm{CFH}$.

${ }^{53}$ If the public party cannot observe the specified construction behaviours, they cannot find out that how much money has been spent by the private firm during last period, which lefts the chance for the private firm to take advantage from the public party by "inflating" the capital expenditure.
} 


\section{5) Lump sum subsidy policies description}

If the public party cannot even observe how many construction stages have been finished by the private firm during each period, the fixed period subsidy policy is no longer available. In this case, a lump sum subsidy policy can be used. That is, the public party only makes a lump sum monetary transfer to the private party proportional to the number of cities that have been finished during each period. For example, if the current state $\{\boldsymbol{a}, \boldsymbol{b}, \boldsymbol{c}, \boldsymbol{d}\}=$ $\{\mathbf{1}, \mathbf{1}, \mathbf{1}, \mathbf{1}\}$ at date 0 , and the firm chooses the construction action $\left\{\boldsymbol{j}_{a^{\prime}}, \boldsymbol{j}_{\boldsymbol{b}}, \boldsymbol{j}_{\boldsymbol{c}^{\prime}}, \boldsymbol{j}_{\boldsymbol{d}}\right\}=\{\mathbf{1}, \mathbf{0}, \mathbf{0}, \mathbf{0}\}$ (which means only city HL will be finished at date 1), CFH only needs to make the lump sum payment to Chorus for the completion of city HL at date 1. Alternatively, the public party is only required to verify the current state $\{\boldsymbol{a}, \boldsymbol{b}, \boldsymbol{c}, \boldsymbol{d}\}$ during each period. ${ }^{54}$ The construction state $\left\{\boldsymbol{j}_{\boldsymbol{a}}, \boldsymbol{j}_{\boldsymbol{b}}, \boldsymbol{j}_{\boldsymbol{c}}, \boldsymbol{j}_{\boldsymbol{d}}\right\}$ becomes irrelevant in this case.

Depending on whether CFH can identify the state of demand for each finished city, we introduce two sorts of lump sum subsidy schemes in our model. A) The independent lump sum subsidy scheme. B) The demand relevant lump sum subsidy scheme. For the first subsidy scheme, the lump sum payment Chorus receives from each completed city is identical. While for the second subsidy scheme, the lump sum payment Chorus receives from a high demand city may be different from what it receives from a low demand city.

\section{6) The independent lump sum subsidy component add-on in evaluation formula}

Being different to the previous two subsidies, the independent lump sum subsidy cash flow occurs only once each city has been finished. That is,

$$
S=\frac{L S}{R_{f}} \times\left(q_{a}+q_{b}+q_{c}+q_{d}\right)
$$

, where $q_{a}=1$ only if $a+j_{a}=$ periodstobuild and $j_{a} \neq 0$ otherwise zero, $q_{b}=1$ only if $b+$ $j_{b}=$ periodstobuild and $j_{b} \neq 0$ otherwise zero, $q_{c}=1$ only if $c+j_{c}=$ periodstobuild and $j_{c} \neq$ 0 otherwise zero and $q_{b}=1$ only if $b+j_{b}=$ periodstobuild and $j_{b} \neq 0$ otherwise zero (13).

$\boldsymbol{L S}$ is the lump sum payment from the public party to the private firm for each finished city. ${ }^{55}$

\footnotetext{
${ }^{54}$ The verification occurs either in the terminal point or whenever the private firm report that they have finished the whole construction. As long as the public party can verify the final state of construction, the private firm will be honest about completeness of project.

${ }^{55}$ The reason we discounted $\boldsymbol{L} \boldsymbol{S}$ by one period risk free rate is because the lump sum payment received by Chorus is one period late after the corresponding city finished.
} 


\section{7) The demand relevant lump sum subsidy component add-on in evaluation formula}

In the case of the demand relevant lump sum subsidy, the subsidy component includes two lump sum payments. That is,

$$
S=\frac{L S 1}{R_{f}} \times\left(q_{a}+q_{b}\right)+\frac{L S 2}{R_{f}} \times\left(q_{c}+q_{d}\right)
$$

, where $q_{a}=1$ only if $a+j_{a}=$ periodstobuild and $j_{a} \neq 0$ otherwise zero, $q_{b}=1$ only if $b+$ $j_{b}=$ periodstobuild and $j_{b} \neq 0$ otherwise zero, $q_{c}=1$ only if $c+j_{c}=$ periodstobuild and $j_{c} \neq$ 0 otherwise zero and $q_{b}=1$ only if $b+j_{b}=$ periodstobuild and $j_{b} \neq 0$ otherwise zero (14).

$\boldsymbol{L S} \mathbf{1}$ is the lump sum payment from the public party to the private firm for finishing a high demand city, while $\boldsymbol{L} \boldsymbol{S} \mathbf{2}$ is the lump sum payment from the public party to the private firm for finishing a low demand city.

\section{8) The process of a comparison of subsidy schemes}

If all four subsidy schemes are available for the public party, CFH may need to know which one is the most socially desirable before they make decisions. Therefore, a comparison among subsidy schemes will be necessary. There are several processes for the comparison. First, we set up four general models, one for the proportional subsidy scheme, one for the fixed period subsidy scheme, and another two for the independent lump sum subsidy scheme and the demand relevant lump sum subsidy scheme respectively. We give all four models the same parameters but with different subsidy components (We use equation 11 to value the proportion subsidy case, equation 12 to value the fixed period subsidy case, equation 13 to value the independent lump sum subsidy case and equation 14 to value the demand relevant lump sum subsidy case). We vary the subsidy variable in each model to minimize the conflicts respectively. ${ }^{56}$ Third, we compare the minimum level of conflicts among all four subsidy schemes to see which one is the smallest. After that, we explain the corresponding differences in minimum conflict level with two conflict measures.

\subsubsection{An add-on of the fine schemes for the general model}

\section{1) The description of fine policies}

\footnotetext{
${ }^{56}$ That is PS for the proportional subsidy scheme, FS for the fixed subsidy scheme, LS for the independent lump sum subsidy scheme and $\{$ LS1, LS2 $\}$ for the demand relevant lump sum subsidy scheme.
} 
In contrast, the public party may introduce a fine policy to discipline the private firm, therefore reducing the potential conflicts. That is, the public party will set up certain targets (Thresholds for the fines) for the private firm in the UFB project. If the private firm achieves the target on time, no fine would be surcharged by the public party, and vice versa. To keep the model simple, we start from a simple fine scheme that the public party will require the private firm to complete all four cities at date 24. If the firm does not achieve the targets, $\mathrm{CFH}$ could require Chorus to pay a fine corresponding to the number of cities not yet finished. The enforcement of the fine policy requires the public party to have at least as much information as the lump sum subsidy, as the public party needs to verify the completeness of the UFB project at date 24. Further on, we allow CFH to switch from a simple fine scheme to a two dimensional fine scheme.

\section{2) The simple fine component add-on in evaluation formula}

Under the simple fine scheme, the penalty only occurs at the date 24 . Therefore, we only need to include the simple fine component to the evaluation formula at date 24 (which is the terminal condition). That is,

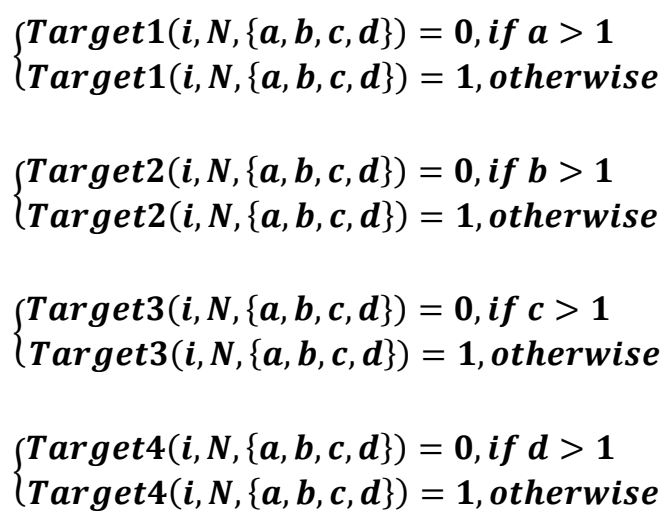

Hence, the whole evaluation equation equals,

$$
\begin{gathered}
F(i, N,\{a, b, c, d\})=\frac{R_{f} R(i, N,\{a, b, c, d\})}{R_{f}-K}-P_{24} \\
\text { where } P_{24}=\text { Finelevel24 } \times(\operatorname{Target1}(i, N,\{a, b, c, d\})+\operatorname{Target}(i, N,\{a, b, c, d\}) \\
+\operatorname{Target} 3(i, N,\{a, b, c, d\})+\operatorname{Target} 4(i, N,\{a, b, c, d\}))(15) .
\end{gathered}
$$

In equation $15, \boldsymbol{P}_{24}$ is the total fine charged by the public party at date 24 (the simple fine component), while Finelevel24 is the penalty that Chorus pays for each unfinished city at date 24 . 


\section{3) The two dimensional fine component add-on in evaluation formula}

In practice, we construct the two dimensional fine scheme by adding an extra group of targets at date 12 to the simple fine scheme. Therefore, we only need to show up the extra fine component. That is,

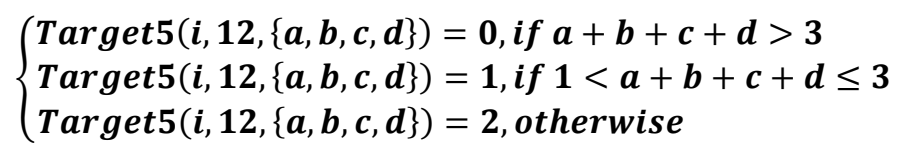

Correspondingly the extra evaluation equation equals

$F(i, 12,\{a, b, c, d\})$

$=\max \left\{R(i, 12,\{a, b, c, d\})-\operatorname{Capex}\left(j_{a}, j_{b}, j_{c}, j_{d}\right)-P_{12}\right.$

$\left.+\frac{\pi_{u}(i, 12) F\left(i, 13,\left\{a+j_{a}, b+j_{b}, c+j_{c}, d+j_{d}\right\}\right)+\pi_{d}(i, 13) F\left(i+1,13,\left\{a+j_{a}, b+j_{b}, c+j_{c}, d+j_{d}\right\}\right)}{R_{f}}\right\}$

where $P_{12}=$ Finelevel12 $\times$ Target $5(i, 12,\{a, b, c, d\})(16)$.

In equation 16, $\boldsymbol{P}_{12}$ is the total amount of the extra fine, while Finelevel12 is the penalty that Chorus pays to $\mathrm{CFH}$ whenever it fails one target at date 12. For the evaluation of the two dimensional fine scheme, we need to combine both equation 15 and equation 16 . 


\section{Chapter 6: The results}

\subsection{The measures of two sorts of conflicts}

Recall that there are two sorts of conflicts between Chorus and CFH in our model. A) The timing conflict indicates that Chorus and CFH may have different opinions about how many cities they would like to build up in each period. B) The sequencing conflict indicates these two parties may have different ideas about choosing the construction order among different cities. In this section, we are going to introduce appropriate measures for each sort of conflict in order to study them separately.

\section{A) The measure for timing conflict}

In this part, we introduce our measure for the timing conflicts, which is based on the expected number of total construction stages completed for certain fixed dates in the future. ${ }^{57}$ That is, for each given fixed date in the future (such as date 4), we first calculate the total number of construction stages expected to be finished when Chorus and $\mathrm{CFH}$ makes the decisions respectively, and then take the difference between them. If the difference turns out to be huge, it means that two parties have huge disagreement on how many construction stages should be finished during a certain time period

In practice, we estimate the expected number of construction stages completed by using the law of iterated expectations. In this approach, at the "terminal" nodes ${ }^{58}$, the expected number of stages completed equals the actual number of completed stages corresponding to the state. For example, at node $(0,4)$, if the state $\{\boldsymbol{a}, \boldsymbol{b}, \boldsymbol{c}, \boldsymbol{d}\}$ equals $\{\mathbf{2}, \mathbf{0}, \mathbf{0}, \mathbf{0}\}$, the expected value equals $\boldsymbol{a}+\boldsymbol{b}+\boldsymbol{c}+\boldsymbol{d}$, which is 2 . However, for any previous (non-terminal) nodes, the expected number of stages that will be completed at the terminal node equals

$E(\boldsymbol{i}, \boldsymbol{n},\{\boldsymbol{a}, \boldsymbol{b}, \boldsymbol{c}, \boldsymbol{d}\})$

$$
\begin{aligned}
& =\theta_{u}(i, n) E\left(i, n+1,\left\{a+j_{a}, b+j_{b}, c+j_{c}, d+j_{d}\right\}\right) \\
& +\theta_{d}(i, n) E\left(i+1, n+1,\left\{a+j_{a}, b+j_{b}, c+j_{c}, d+j_{d}\right\}\right)
\end{aligned}
$$

where $j_{a}, j_{b}, j_{c}$ and $j_{d}$ are the corresponding construction policies for each city (15)

\footnotetext{
${ }^{57}$ The fixed dates we choose are date 4 (the first year), date 8 (the second year), date 12 (the third year), date 16 (the fourth year), date 20 (the fifth year) and date 24 (the last year). The above selected dates are arbitrary. ${ }^{58}$ The terminal nodes here are the nodes only corresponding to the fixed dates. For example, if we take date 4 as the fixed date, all nodes at date 4 will be the terminal nodes.
} 
In this equation, $\boldsymbol{E}(\boldsymbol{i}, \boldsymbol{n},\{\boldsymbol{a}, \boldsymbol{b}, \boldsymbol{c}, \boldsymbol{d}\})$ is the expected value current node $(\mathbf{i}, \mathbf{n})$.

$\boldsymbol{E}\left(\boldsymbol{i}, \boldsymbol{n}+\mathbf{1},\left\{\boldsymbol{a}+\boldsymbol{j}_{\boldsymbol{a}}, \boldsymbol{b}+\boldsymbol{j}_{\boldsymbol{b}}, \boldsymbol{c}+\boldsymbol{j}_{\boldsymbol{c}}, \boldsymbol{d}+\boldsymbol{j}_{\boldsymbol{d}}\right\}\right)$ is the expected value at next period when there has been one up move, while $\boldsymbol{E}\left(\boldsymbol{i}+\mathbf{1}, \boldsymbol{n}+\mathbf{1},\left\{\boldsymbol{a}+\boldsymbol{j}_{\boldsymbol{a}}, \boldsymbol{b}+\boldsymbol{j}_{\boldsymbol{b}}, \boldsymbol{c}+\boldsymbol{j}_{c}, \boldsymbol{d}+\boldsymbol{j}_{\boldsymbol{d}}\right\}\right)$ is the expected value next period when there has been one down move. $\boldsymbol{\theta}_{\boldsymbol{u}}$ and $\boldsymbol{\theta}_{\boldsymbol{d}}$ have been introduced here as the real probabilities of up and down moves occurring in the next period respectively, where $\boldsymbol{\theta}_{\boldsymbol{u}}(\boldsymbol{i}, \boldsymbol{n})$

equals $\frac{1}{2}+\frac{\mu \sqrt{d t}}{2 \sigma}$ and $\theta_{d}(i, n)$ equals $\frac{1}{2}-\frac{\mu \sqrt{d t}}{2 \sigma}$ (See Guthrie (2009) for the corresponding derivation). We first calculate the expected value at all terminal points and then use the backward induction (using equation 15) to get the expected values at all previous periods. We report the expected number of construction stages completed for the conflict in the baseline case in which there are no incentive scheme at the panel B of table 6.1.

\section{B) The measure for sequencing conflict}

In this section, we introduce our measure for the sequencing conflict. The measure we apply to estimate the sequencing conflict is based on a table of conditional probabilities. In a probability table (panel $\mathrm{C}$ in table 6.1), there are two panels. The first one records probabilities related to Chorus's decisions, while the second one records probabilities related to $\mathrm{CFH}$ decisions. For each panel, the rows represent four different cities (HL, HH, LL and LH), while the columns represent the states of completion for each given city. More specifically, the first column denotes the city has been finished, whereas the following four columns denote all possible orders of completion for this city (First, Second, Third, Fourth). Therefore, each cell in the first column represents the probability that a corresponding city (rows) will be finished eventually, while each cell in the following four columns represents the conditional probability that a corresponding city (rows) will be finished in a corresponding order (columns) providing this city will be finished eventually.

In practice, we need to calculate the unconditional probabilities that a city will be finished in a particular order before we can estimate the conditional probabilities (column 2 to column 5) and the probability for completion (the first column). In much the same way as calculating the expected number of construction stages completed, we apply the law of iterated expectations to estimate all unconditional probabilities. That is, we design a bundle of lotteries that have payoff equal to 1 whenever a corresponding city has just been finished in a particular order and payoff equal to zero when it is finished in a different order or not finished at all. In order to make the present values of lotteries equal the values of corresponding unconditional probabilities, we set the discount rate to zero. At the terminal 
condition $^{59}$, the payoffs of all lotteries equal 0 since none of the parties are allowed to have construction action when the contract expires. However, for any non-terminal nodes, the value of the lottery equals

$L_{X Y}(i, n,\{a, b, c, d\})$

$(1$, if the city $X$ has been finished in $Y$ order at node $(i, n)$

$=\left\{\begin{array}{l}\theta_{u}(i, n) L_{X Y}\left(i, n+1,\left\{a+j_{a}, b+j_{b}, c+j_{c}, d+j_{d}\right\}\right)+\theta_{d}(i, n) L_{X Y}\left(i+1, n+1,\left\{a+j_{a}, b+j_{b}, c+j_{c}, d+j_{d}\right\}\right) \\ \text { otherwise }\end{array}\right.$

where $j_{a}, j_{b}, j_{c}$ and $j_{d}$ are the corresponding construction policies for each city (16)

In equation $16, \boldsymbol{L}_{X Y}(\boldsymbol{i}, \boldsymbol{n},\{\boldsymbol{a}, \boldsymbol{b}, \boldsymbol{c}, \boldsymbol{d}\})$ is the value of the lottery that city $\mathrm{X}$ has been finished in order Y at node $(\boldsymbol{i}, \boldsymbol{n}) . \boldsymbol{L}_{X Y}\left(\boldsymbol{i}, \boldsymbol{n}+\mathbf{1},\left\{\boldsymbol{a}+\boldsymbol{j}_{\boldsymbol{a}^{\prime}} \boldsymbol{b}+\boldsymbol{j}_{\boldsymbol{b}^{\prime}} \boldsymbol{c}+\boldsymbol{j}_{\boldsymbol{c}^{\prime}} \boldsymbol{d}+\boldsymbol{j}_{\boldsymbol{d}}\right\}\right)$ is the value of the lottery next period when there has been one up move, while $\boldsymbol{L}_{X Y}\left(\boldsymbol{i}+\mathbf{1}, \boldsymbol{n}+\mathbf{1},\left\{\boldsymbol{a}+\boldsymbol{j}_{\boldsymbol{a}^{\prime}} \boldsymbol{b}+\boldsymbol{j}_{\boldsymbol{b}^{\prime}} \boldsymbol{c}+\boldsymbol{j}_{\boldsymbol{c}^{\prime}} \boldsymbol{d}+\right.\right.$ $\left.\left.\boldsymbol{j}_{\boldsymbol{d}}\right\}\right)$ is the value of the lottery next period when there has been one down move. After the terminal conditions and backward induction (using equation 16), we can get the expected value of lottery XY $\left(\boldsymbol{L}_{X Y}(\mathbf{0}, \mathbf{0},\{\mathbf{0}, \mathbf{0}, \mathbf{0}, \mathbf{0}\})\right)$, which is the unconditional probability that city $\mathrm{X}$ has been finished in $\mathrm{Y}$ order. Then, we use equation 17 to get the probability of city $\mathrm{X}$ being finished eventually,

$$
\mathbf{L}_{\mathrm{Xcompleted}}(\mathbf{0}, \mathbf{0},\{\mathbf{0}, \mathbf{0}, \mathbf{0}, 0\})=\sum_{\mathrm{Y}=1}^{\mathrm{Y}=4} \mathbf{L}_{\mathrm{XY}}(\mathbf{0}, \mathbf{0},\{\mathbf{0}, \mathbf{0}, \mathbf{0}, \mathbf{0}\})
$$

Therefore, the conditional probability that city $\mathrm{X}$ will be finished in $\mathrm{Y}$ order providing it will be finished eventually equals,

$$
\mathbf{L}_{\mathbf{X Y C o n d i t i o n a l}}(\mathbf{0}, \mathbf{0},\{\mathbf{0}, \mathbf{0}, \mathbf{0}, \mathbf{0}\})=\frac{\mathbf{L}_{\mathbf{X Y}}(\mathbf{0}, \mathbf{0},\{\mathbf{0}, \mathbf{0}, \mathbf{0}, \mathbf{0}\})}{\mathbf{L}_{\mathbf{X} \text { completed }}(\mathbf{0}, \mathbf{0},\{\mathbf{0}, \mathbf{0}, \mathbf{0}, \mathbf{0}\})}(18)
$$

We provide an example of a probability table for the conflict in the baseline case in which there are no incentive scheme (which is at the panel $\mathrm{C}$ of table 6.1).

\subsection{The conflicts between two parties in the baseline case}

Before we start analysing incentive schemes, we discuss the profile of the conflicts between Chorus and $\mathrm{CFH}$ in the baseline case in which there is no incentive scheme. To get the profile of the conflicts, we follow several procedures. First, we apply the valuation processes that we develop at section 5.3 to estimate the present value of total surplus of the UFB project for Chorus under Chorus's optimal policy $(\boldsymbol{F}(\mathbf{0}, \mathbf{0},\{\mathbf{0}, \mathbf{0}, \mathbf{0}, \mathbf{0}\}))$, the present value of

${ }^{59}$ We only take the nodes at date 24 as terminal condition. 
“producer” surplus of the UFB project for CFH under CFH's optimal policy $(\boldsymbol{T}(\mathbf{0}, \mathbf{0},\{\mathbf{0}, \mathbf{0}, \mathbf{0}, \mathbf{0}\}))$ and the present value of the UFB project for CFH under Chorus's optimal policy $(\boldsymbol{G}(\mathbf{0}, \mathbf{0},\{\mathbf{0}, \mathbf{0}, \mathbf{0}, \mathbf{0}\}))$. Then, we input $\boldsymbol{T}(\mathbf{0}, \mathbf{0},\{\mathbf{0}, \mathbf{0}, \mathbf{0}, \mathbf{0}\})$ and $\boldsymbol{G}(\mathbf{0}, \mathbf{0},\{\mathbf{0}, \mathbf{0}, \mathbf{0}, \mathbf{0}\})$ into equation 11 to calculate the present value of conflict and equation 12 to calculate the proportion of conflict.

Table 6.1: The profile of the conflicts in the baseline case

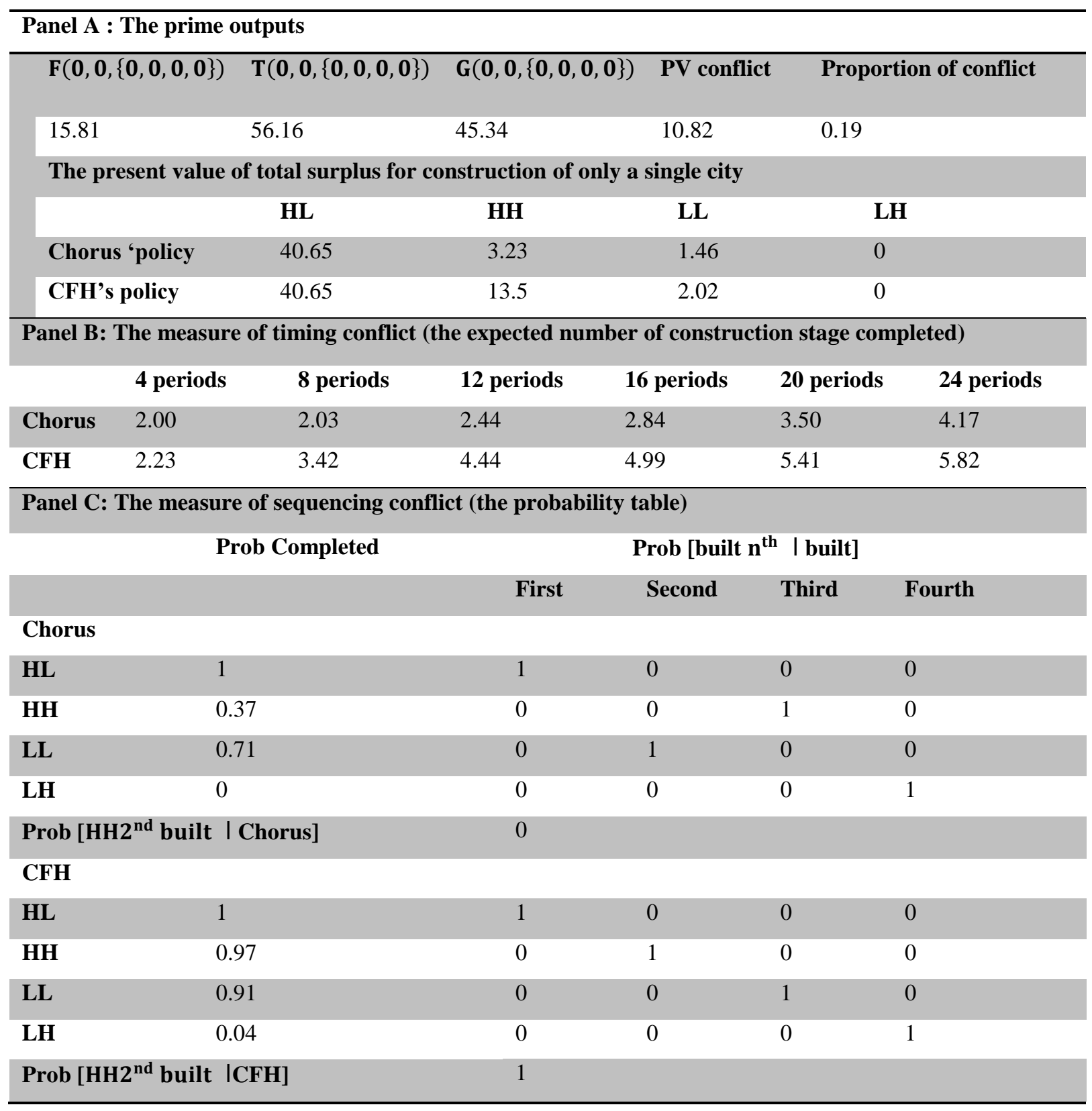

We estimate the net present value of each single city under both CFH's and Chorus's policies in order to identify which city contributes the most to the level of total conflict. 
After that, we use both the timing conflict and sequencing conflict measures to summarize the corresponding sequencing and timing conflicts. All parameters we apply in the above procedures are from table 5.1. We report the outputs in table 6.1 .

From panel A of table 6.1, we can learn that the present value of the UFB project for CFH when Chorus's optimal policies have been adopted equals 45.34, while the present value of the UFB project for CFH when CFH's optimal policies have been adopted equals 56.16. The difference between these two present values equals 10.82, which is the present value of conflict. We may interpret it as the present value of potential social welfare loss resulting from the disagreement between the two parties on optimal policies. ${ }^{60}$ This is because, assuming that $\mathrm{CFH}$ works in the best interests of society, its optimal policy should always maximise social welfare. Therefore, any policy which deviates from CFH's optimal policy may bring a potential social welfare loss, where these welfare losses may be raised either from an undersupply in the UFB network during certain periods ${ }^{61}$ or an incorrect priority policy among different cities. ${ }^{62}$ The conflicts between two parties have created 19.3 per cent of social welfare loss. When we compare the present value of total surplus for constructing a single city, we find that the conflicts in the baseline case (in which there are no incentive scheme) are mainly attributed to the disagreement between two parties on city $\mathrm{HH}^{63}$

Panel B reports the expected number of construction stages completed for both parties during given periods. In four periods, CFH expects to finish about 2.23 construction stages, while Chorus only expects to finish 2.00 construction stages. When we extend the periods from four to sixteen, the numbers of construction stages $\mathrm{CFH}$ would like to finish jumps to about 5, while the number for Chorus increases to about 2.84. Further, when we extend the periods to 24, the number for $\mathrm{CFH}$ jumps to about 5.8, while the number for Chorus increases to around 4.2. Clearly, there are disagreements on construction timing between two parties, that is, $\mathrm{CFH}$ is more aggressive than Chorus in building up the UFB network. The logic behind this result is quite clear when we recall the differences of the two parties' objective functions. As we know, for each completed city, $\mathrm{CFH}$ always receives consumer surplus plus producer surplus, while Chorus only receives producer surplus. The extra

\footnotetext{
${ }^{60}$ The difference is a consequence of the two parties' disagreement on optimal policies.

${ }^{61}$ The firm may work too slowly to provide enough supply to meet the social demand.

${ }^{62}$ For saving the cost, the firm may give priority to a low cost city instead of a high cost city, even though the high cost city may have a higher total surplus than that of the low cost city.

${ }^{63}$ About $94 \%$ of the baseline conflicts result from city $\mathrm{HH}$.
} 
surplus flow makes construction more profitable to $\mathrm{CFH}$ than Chorus. Therefore $\mathrm{CFH}$ always wants to build up more cities.

In panel $\mathrm{C}$, a comparison of conditional probabilities between Chorus and $\mathrm{CFH}$ illustrates their sequencing conflicts. Both parties agree that city HL should be the first city to be completed. However, after city HL has been completed, $\mathrm{CFH}$ and Chorus disagree on which city should go next, that is, $\mathrm{CFH}$ wants to build up city $\mathrm{HH}$ second providing that at least two cities (both city HL and city HH) are built up eventually (with conditional probability of 1), while Chorus would like to build up city LL second instead providing that at least two cities (both city HL and city LL) are built up eventually (with conditional probability of 1 as well). ${ }^{64}$ Namely, a sequencing conflict arises between two parties when they are deciding which city should go second. Similarly, the two parties disagree on which city should go third. After finishing city HL and city HH, CFH certainly wants to finish city LL third (with conditional probability of 1) providing that at least three cities will be finished eventually. In contrast, Chorus wants to finish city HH third (with conditional probability of 1) providing that at least three cities will be finished eventually. For city LH, both parties consider it should be granted the lowest priority, which means that if city LH would be finished eventually it must go last. Table 6.2 is a summary for the construction behaviours and sequencing conflicts between $\mathrm{CFH}$ and Chorus.

\section{Table 6.2: Construction order for each party}

\begin{tabular}{|c|c|c|c|}
\hline & \multicolumn{2}{|c|}{ The construction orders } & \multirow{2}{*}{$\begin{array}{l}\text { Sequencing Conflicts } \\
\text { (Yes/No) }\end{array}$} \\
\hline & CFH & Chorus & \\
\hline If one city has been built up & HL & HL & No \\
\hline If two cities have been built up & $\mathrm{HL} \rightarrow \mathrm{HH}$ & $\mathrm{HL} \rightarrow \mathrm{LL}$ & Yes \\
\hline If three cities have been built up & $\mathrm{HL} \rightarrow \mathrm{HH} \rightarrow \mathrm{LL}$ & $\mathrm{HL} \rightarrow \mathrm{LL} \rightarrow \mathrm{HH}$ & Yes \\
\hline If four cities have been built up & $\mathrm{HL} \rightarrow \mathrm{HH} \rightarrow \mathrm{LL} \rightarrow \mathrm{LH}$ & $\mathrm{HL} \rightarrow \mathrm{LL} \rightarrow \mathrm{HH} \rightarrow \mathrm{LH}$ & Yes \\
\hline
\end{tabular}

As a measure of sequencing conflict, the conditional probabilities could be applied to not only identify the conflict but also show the "scale" of conflict. However, we need to control for the timing conflicts when we interpret the "scale" of sequencing conflicts. For example, if there are two cities going to be finished for both parties (no timing conflict), the difference

\footnotetext{
${ }^{64}$ The conditional probabilities that CFH builds up city LL second providing that both city HL and city LL have been built up eventually and Chorus builds up city $\mathrm{HH}$ second providing that both city $\mathrm{HL}$ and city $\mathrm{HH}$ have been built up eventually are zero since those behaviours never occur.
} 
between conditional probabilities that CFH builds up city $\mathrm{HH}$ second and Chorus builds up city $\mathrm{HH}$ second are $1,{ }^{65}$ which means that Chorus and $\mathrm{CFH}$ will have 100 per cent chance to disagree on the order of city completion (which is the maximum scale of sequencing conflict in our model). On the other hand, if this difference is smaller, the sequencing conflict between these two parties is smaller.

In common with the timing conflicts, the different preferences on building up the second city between $\mathrm{CFH}$ and Chorus are triggered by the consumer surplus cash flow. With this extra cash flow, the capital expenditure plays a smaller role in CFH's present value than that of Chorus, which renders CFH less cost focused than Chorus. (In other words, CFH is more demand focused than Chorus.) Therefore, after the most profitable city, HL, has been finished, $\mathrm{CFH}$ would like to give priority to a high demand city (city $\mathrm{HH}$ ), while Chorus would like to give priority to a low cost city (city LL).

\subsection{The sensitivity analysis on the conflicts in the baseline case}

In this section we report the results of sensitivity analysis on the conflicts in the baseline case in which there is no incentive scheme. That is, we study how the present value of conflict is affected by varying several parameters $(\boldsymbol{D R}, \boldsymbol{C R}, \boldsymbol{C o n}$ or $\boldsymbol{\sigma})$. We also apply the two measures of conflicts to interpret the results of our sensitivity analysis.

\subsubsection{The sensitivity analysis on Con}

Con is the constant multiplier between consumer surplus and the square of producer surplus. In contrast to the demand and cost multipliers, the present value of conflict monotonically increases with Con. We report how the conflict level varies with Con at graph 6.1. As we can see, the present value of conflict is almost a convex curve with Con at graph 6.1.

The logic behind this result is straightforward. When we raise Con, for any given producer surplus flow, the consumer surplus flow increases. Since raising the consumer surplus flow reduces the proportion of producer surplus in the total surplus, $\mathrm{CFH}$ and Chorus share less similarity in their economic interests. Therefore, the conflict between these two parties became bigger.

We justify our explanation about the raising conflict by employing the two conflict measures. As we can see from graph 6.2, the conditional probability that Chorus would like to

${ }^{65}$ Which equals the difference between $\operatorname{Prob}\left[\mathrm{HH} 2^{\text {nd }}\right.$ built $\left.\mid \mathrm{CFH}\right]$ and $\operatorname{Prob}\left[\mathrm{HH} 2^{\text {nd }}\right.$ built $\left.\mid \mathrm{CFH}\right]$. 
complete city $\mathrm{HH}$ second providing that at least two cities will be finished always equals zero, while the corresponding conditional probability for CFH monotonically increases with Con. Therefore, the scale of sequencing conflict (which is the difference between the conditional probability of $\mathrm{CFH}$ and the conditional probability of Chorus) is monotonic increasing with Con.

\section{Graph 6.1: The present value of conflict varies with Con}

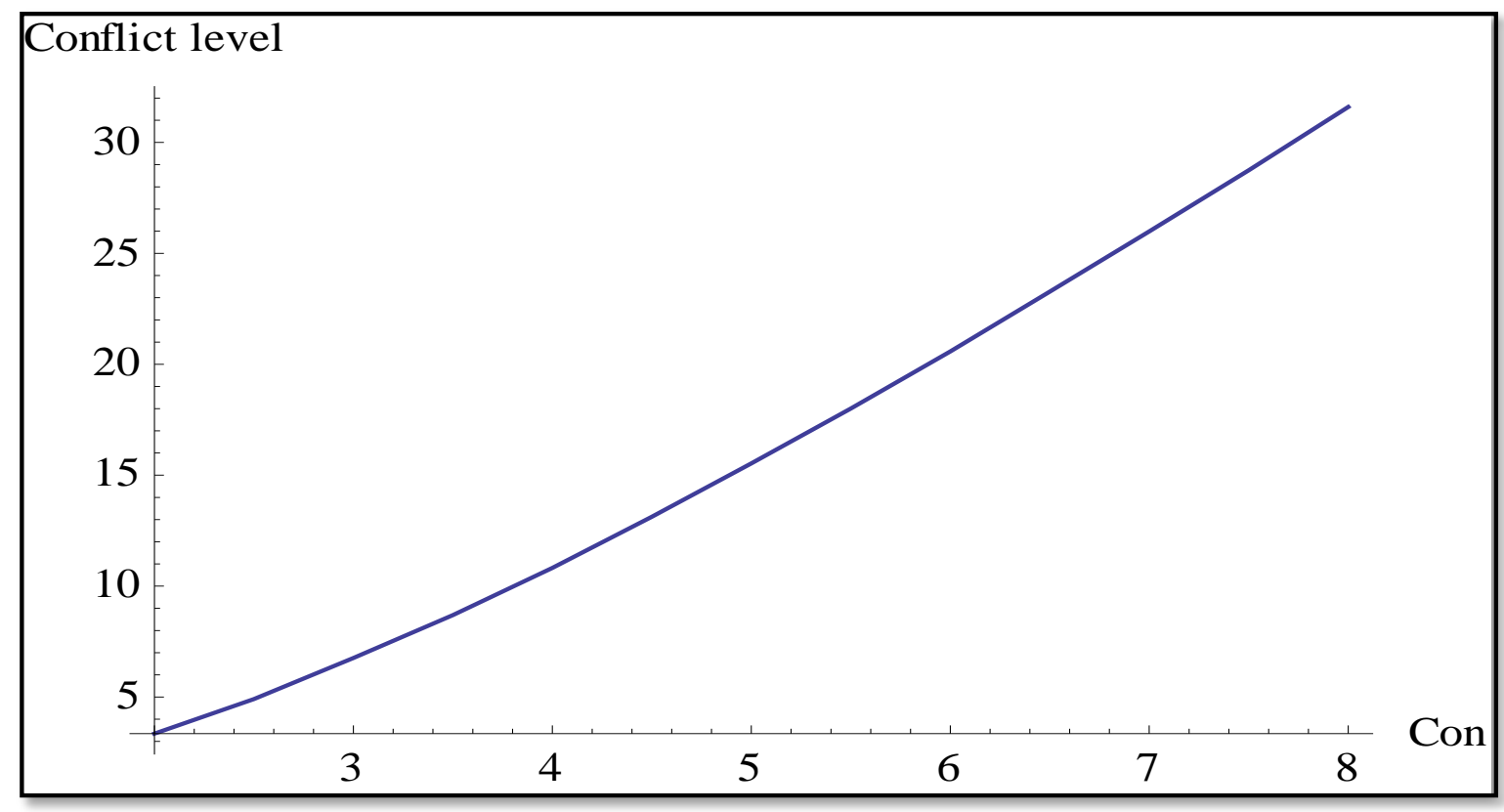

Graph 6.2: The sequencing conflict measures varies with Con

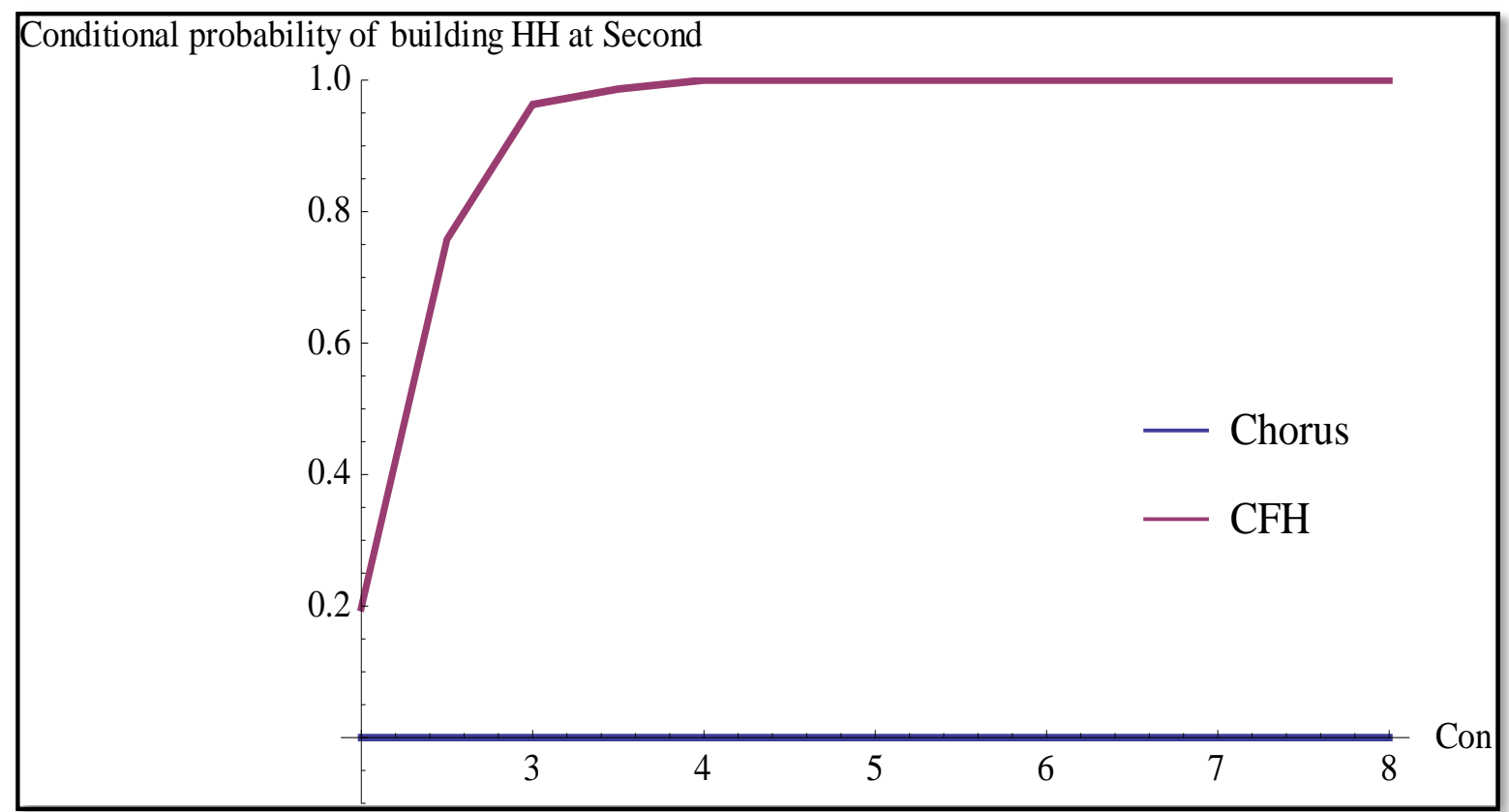




\section{Graph 6.3: The timing conflict measures varies with Con}

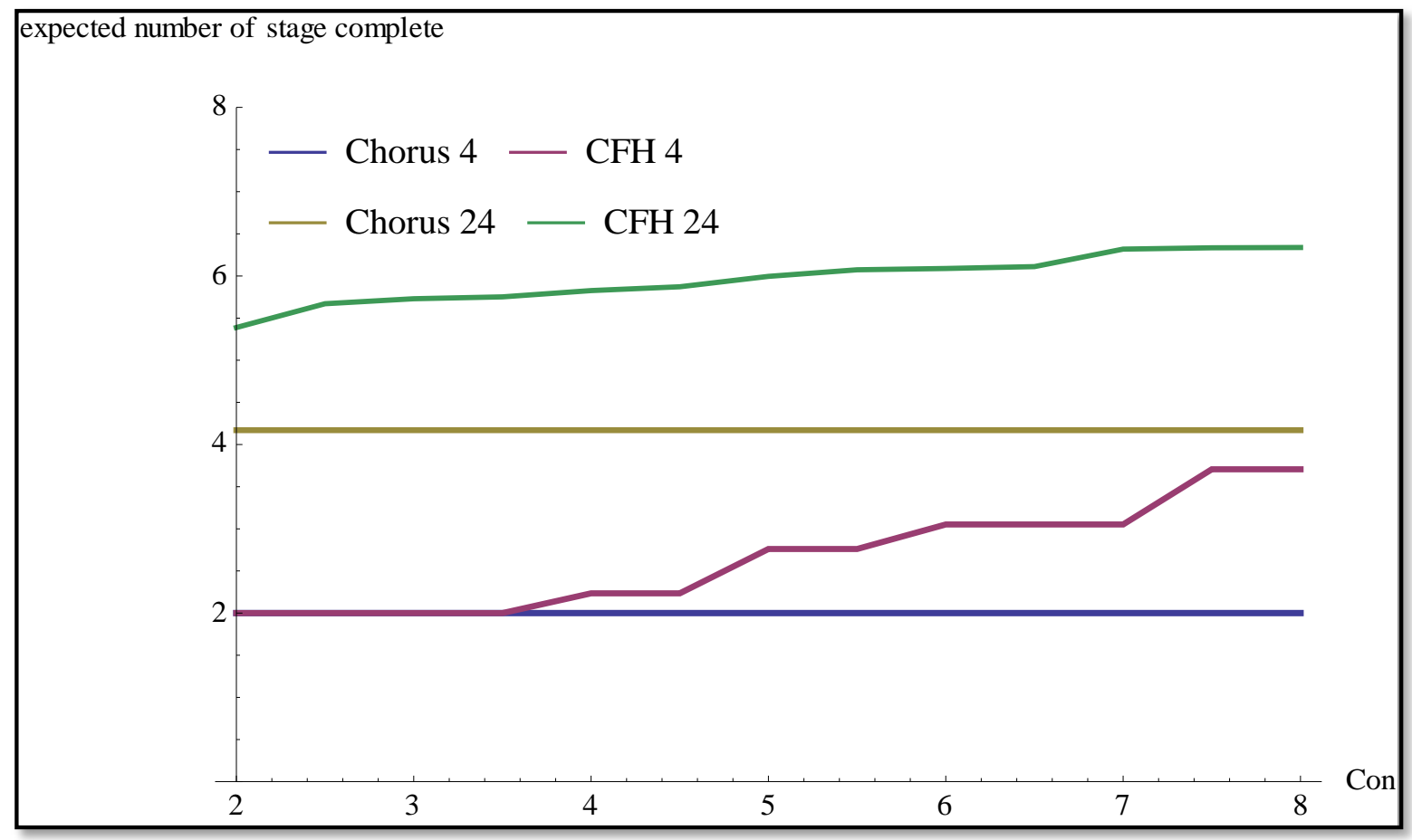

At graph 6.3, we apply the timing conflict measure to two target dates in order to justify the movement of conflict level over Con. That is, we estimate both the expected numbers of construction stages completed in 4 periods and 24 periods. The expected number in 4 periods gives us information about the timing differences between $\mathrm{CFH}$ and Chorus of those highly profitable cities, while the expected number in 24 periods adds the timing information about those marginal cities. ${ }^{66}$ We find that, for whichever target date we consider, the difference of

\footnotetext{
${ }^{66}$ For both $\mathrm{CFH}$ and Chorus, the profitable cities tend to be finished at the early stage, while the marginal cities would not be finished unless the contract is near expiration. Therefore, the difference of the expected number based on the expiration date (at date 24) is not able to capture the timing differences for those highly profitable cities between $\mathrm{CFH}$ and Chorus (since the high profitable cities will be finished by both parties before contract expire anyway). In contrast, the difference of the expected number based on the early stage (like 4 periods) is able to record the timing differences for those highly profitable cities but has nothing to say about the timing differences for those marginal cities (since neither party will develop the marginal cities in the early stage). An extreme case for the profitable city is city HL. As we can see from graph 6.3, both parties would like to finish city HL in 4 periods no matter how Con changes (at graph 6.3, the expected numbers of construction stages completed in 4 periods are equal or bigger than 2 for both parties. This indicates either at least one city has been finished or at least two cities are under construction. Since both parties want to construct city $\mathrm{HL}$ first due to its profitability, and neither party wants to interrupt the construction of city $\mathrm{HL}$ in order to start the construction of another city (parties only receive revenues from the completed cities), we believe city $\mathrm{HL}$ will be finished in 4 periods). Therefore, although the expected numbers of city $\mathrm{HL}$ equal 2 for both 4 periods and 24 periods, the 4 periods measure provides us more information about city HL's timing since it not only tells us whether city HL would be finished before the contract expires but also how fast city $\mathrm{HL}$ would be completed. In contrast, city LH will be an extreme case for the marginal city since it would not be started unless other three cities have been finished. The expected number in 4 periods tells us nothing about the timing of city LH since it is nearly impossible for any party to start city LH in 4 periods. However, the
} 
the expected number of construction stages completed between $\mathrm{CFH}$ and Chorus is monotonic increasing with Con. Hence, the timing conflict between the two parties is increasing with Con. In summary, raising Con increases both the timing and sequencing conflicts between two parties, therefore resulting in a higher level of total conflict between two parties.

\subsubsection{The sensitivity analysis on volatility $(\sigma)$}

At graph 6.4, we find that the present value of conflict exponentially increases with volatility. When we raise the level of volatility from 0.1 to 0.15 , the present value of conflict goes up from 4.24 to 10.8. After reaching the middle point $(\sigma=0.15)$, the conflict level starts to increase far more quickly. That is, the conflict level jumps from 10.8 to 69 when we increase the volatility level from 0.15 to $0.2 .^{67}$

\section{Graph 6.4: The present value of conflict varies with $\sigma$}

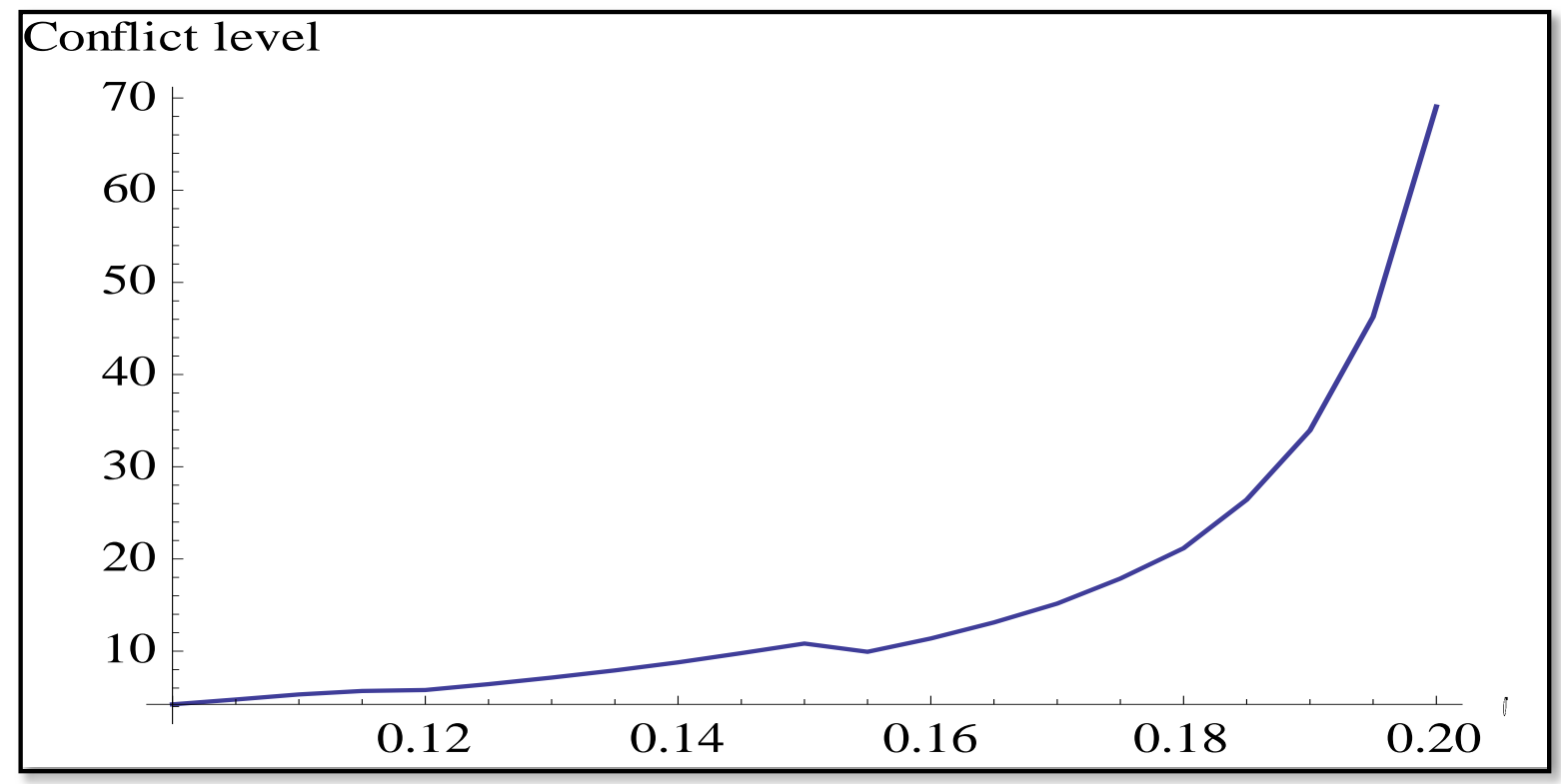

At graph 6.5, we find that the conditional probabilities that city $\mathrm{HH}$ will be completed second providing that at least two cities will be finished eventually for both parties are constant over $\sigma$. In other words, the sequencing conflict between two parties hardly changes

expected number in 24 periods at least tells us how many stages of city LH are expected to be finished before the contract expires. The profitability of city $\mathrm{HH}$ and city $\mathrm{LL}$ is between city $\mathrm{HL}$ and city LH.

${ }^{67}$ Our model does not work properly when volatility is above a certain level. This is because the growth rate of consumer surplus flow is an increasing function of volatility in our setting. When the volatility goes above a certain level, we find the growth rate of consumer surplus is higher than the corresponding discount rate, so that the present value of consumer surplus is undefined. Therefore, we cap annual volatility at 0.2 in our study, which we believe it is a realistic level of volatility for the internet business. 
when we vary the level of volatility. Hence, the movement of the conflict level must be mainly driven by these two parties' timing disagreements.

\section{Graph 6.5: The sequencing conflict measures varies with $\sigma$}

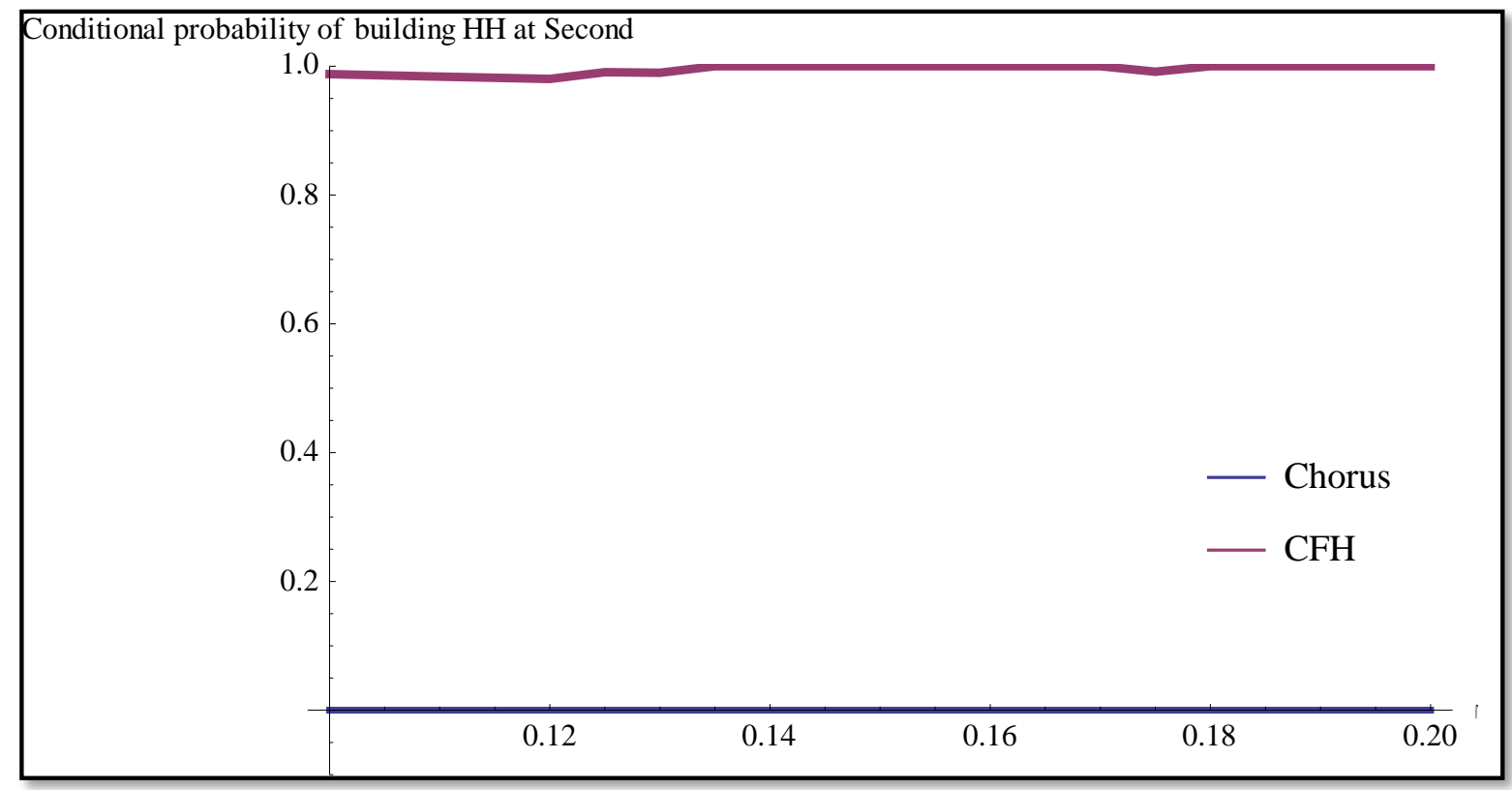

Graph 6.6: The timing conflict measures varies with $\sigma$

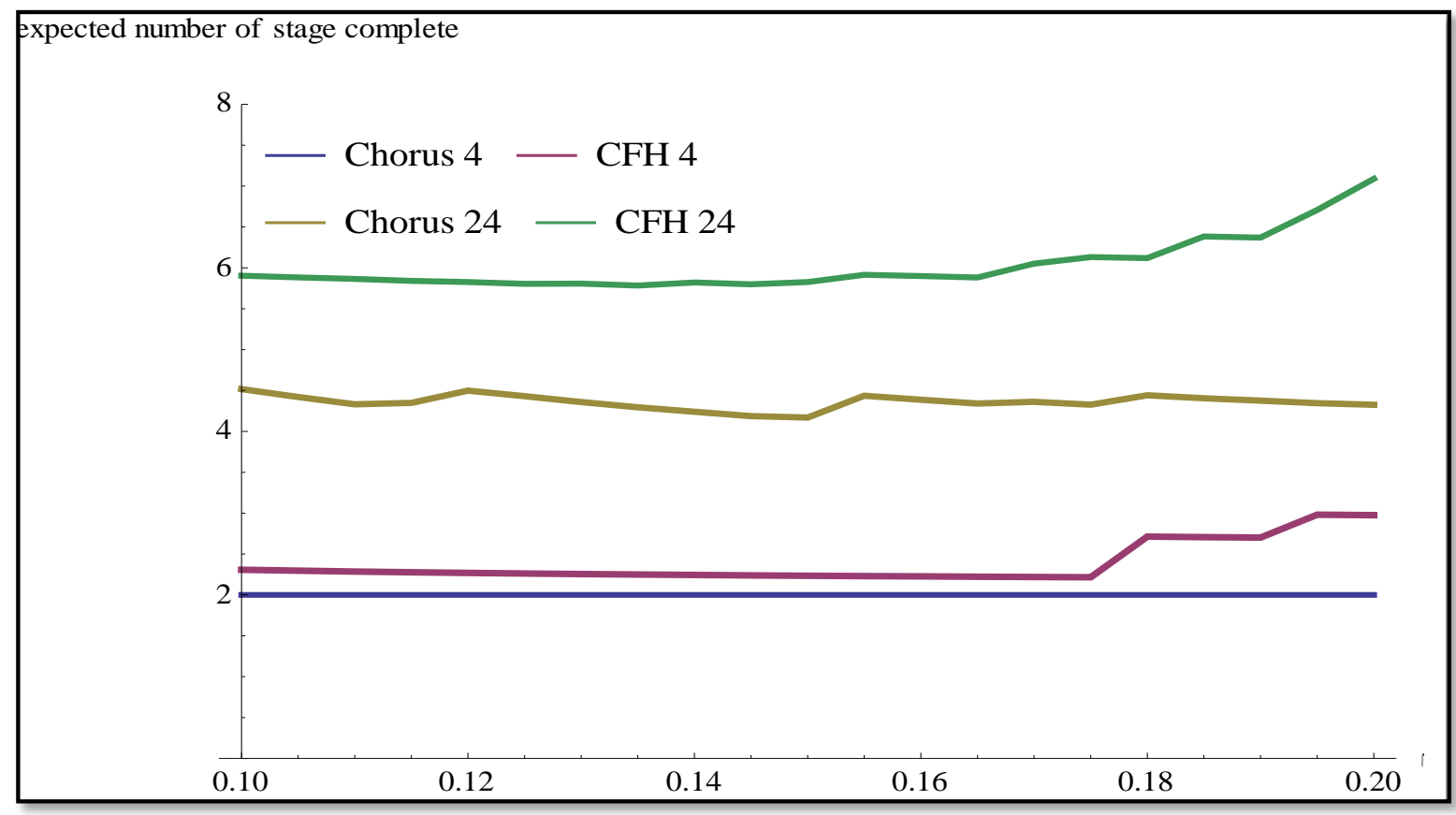

At graph 6.6, we find that the timing conflict between Chorus and $\mathrm{CFH}$ grows over the level of volatility. More specifically, the differences of the expected number of construction stages completed in both 4 and 24 periods are increasing with $\sigma$. In summary, although the increase 
of volatility does not change the sequencing conflict, it does aggravate the timing conflict therefore increasing the level of total conflict between two parties. Besides, the increase of conflict level may be partially attributed to the increase of construction option value. For example, when we increase $\sigma$ from 0.1 to 0.2 , the present value average of total surplus on constructing city $\mathrm{HH}$ under two parties' policies increases from 2.3 to 66.5 (clearly, the option value increases with volatility). ${ }^{68}$

\subsubsection{The sensitivity analysis on $D R$ and $C R$}

We conduct the sensitivity analysis for both demand and cost multipliers ( $\boldsymbol{D R}$ and $\boldsymbol{C R})$ in this section since they share several similarities in their effects on the present value of conflict. Recall that $\boldsymbol{D R}$ is the demand multiplier that we apply to the revenue of a standard city to form the high demand city, while $\boldsymbol{C R}$ is the cost multiplier that we apply to the capital expenditure of a standard city to form the high cost city. We report how the present value of conflict varies with demand and cost multipliers at graph 6.7 and 6.8 respectively. Because of limited space, we only investigate several selective $\boldsymbol{D R}$ and $\boldsymbol{C R}{ }^{69}$

\section{Graph 6.7: The present value of conflict varies with $D R$}

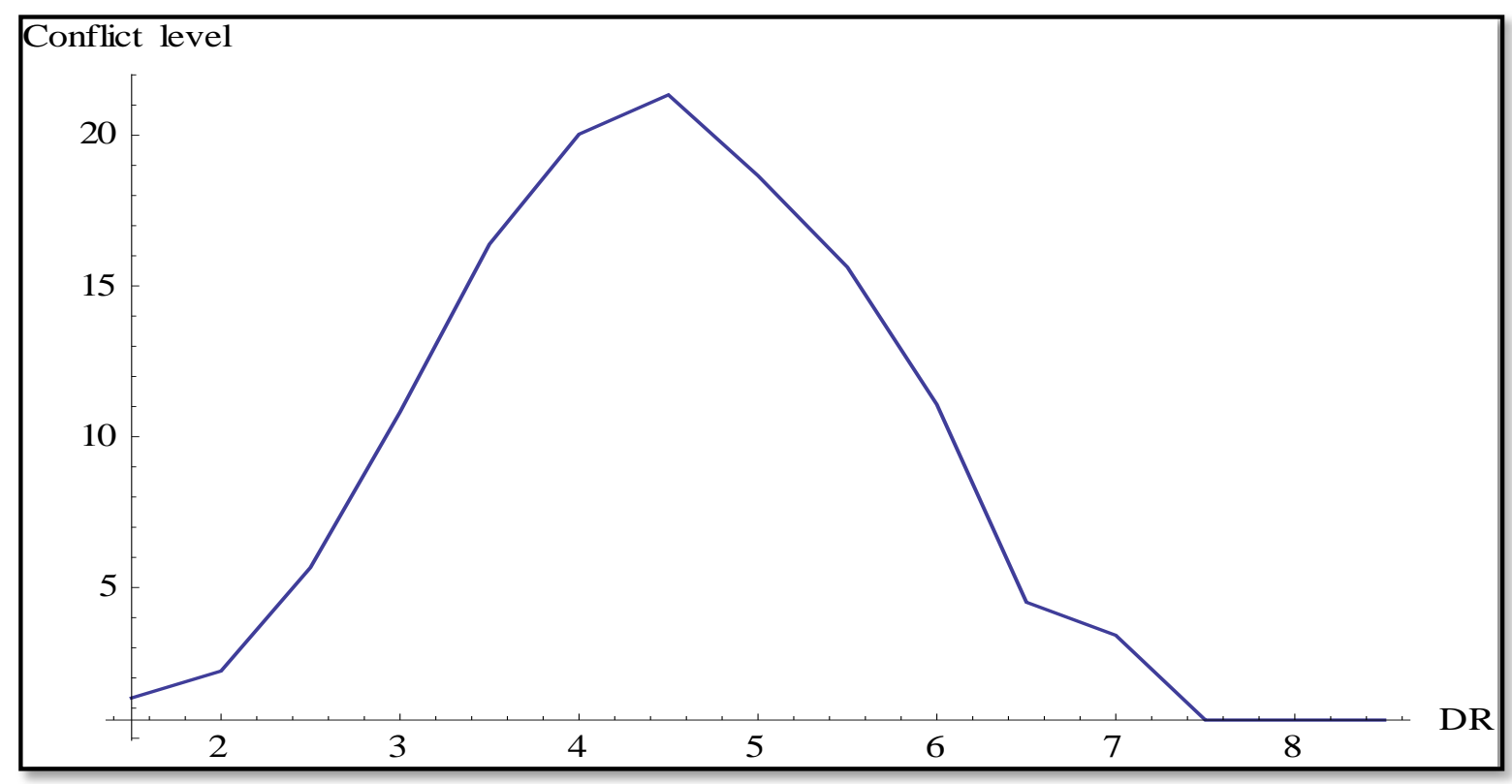

\footnotetext{
${ }^{68}$ We take the arithmetic average of the present value of total surplus on constructing city $\mathrm{HH}$ under $\mathrm{CFH}$ and Chorus's policies. The change of this average gives us a clue about how the magnitude of conflict changes over volatility.

${ }^{69}$ For both DR and CR, we start the sensitivity analysis from the baseline case, that is $D R=3$ and $C R=2$. And then, we investigate how the level of conflicts in the baseline case changes when we change DR or CR.
} 
Graph 6.7 shows the relationship between conflict level and $\boldsymbol{D R}$ is non-monotonic, that is, the conflict level first increases and then decreases over $\boldsymbol{D R}$. We observe the same nonmonotonic movement of conflict level when we vary the cost multiplier at graph 6.8.

\section{Graph 6.8: The present value of conflict varies with $C R$}

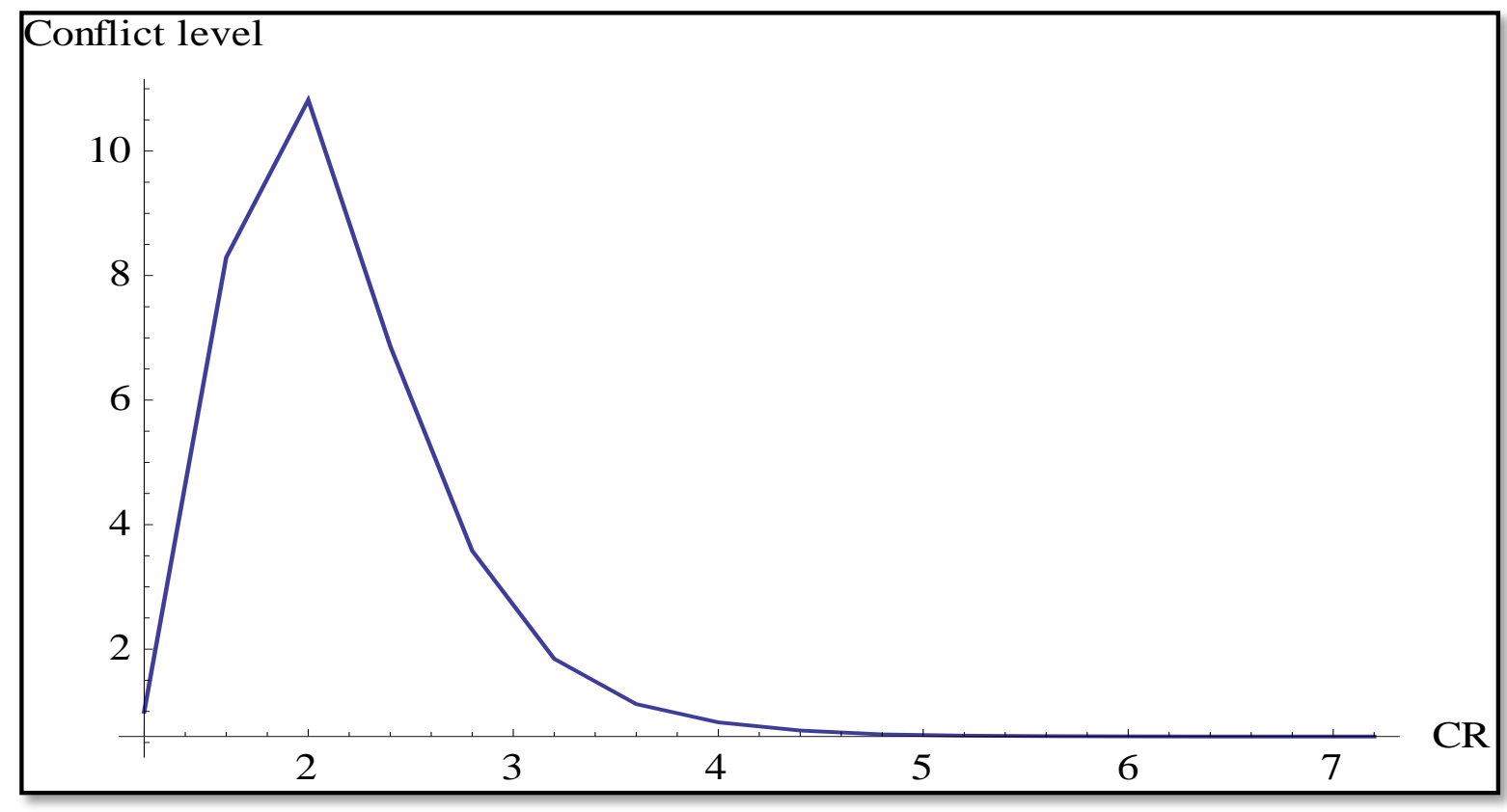

Now, we discuss the effects of both $\boldsymbol{D R}$ and $\boldsymbol{C R}$ on the conflict level by analysing timing and sequencing conflict measures. The corresponding information for these two conflict measures has been reported at graph 6.9, graph 6.10, graph 6.11 and graph 6.12. From graph 6.9 and 6.10, we find that the sequencing conflicts only exist for certain ranges of $\boldsymbol{D R}$ or $\boldsymbol{C R}$. For example, controlling for $\boldsymbol{C R}$ at graph 6.9, both parties agree to finish the city LL before city $\mathrm{HH}$ when the demand multiplier is smaller than 2.5 (the conditional probabilities of building city $\mathrm{HH}$ second are equal to zero for both parties at graph 6.9). However, when we range $\boldsymbol{D} \boldsymbol{R}$ between 2.5 and 4, we identify a sequencing conflict which is raised by the discordant shifts of construction preference between two parties. That is, although both parties would like to shift their construction preferences over which city should be finished second from city LL to city $\mathrm{HH}$, the preference of $\mathrm{CFH}$ changes faster than that of Chorus. Further on, if we make $\boldsymbol{D} \boldsymbol{R}$ bigger than 4 , the sequencing conflict disappears and both parties agree to finish the city HH before city LL. 
Graph 6.9: The sequencing conflict measure varies over $D R$

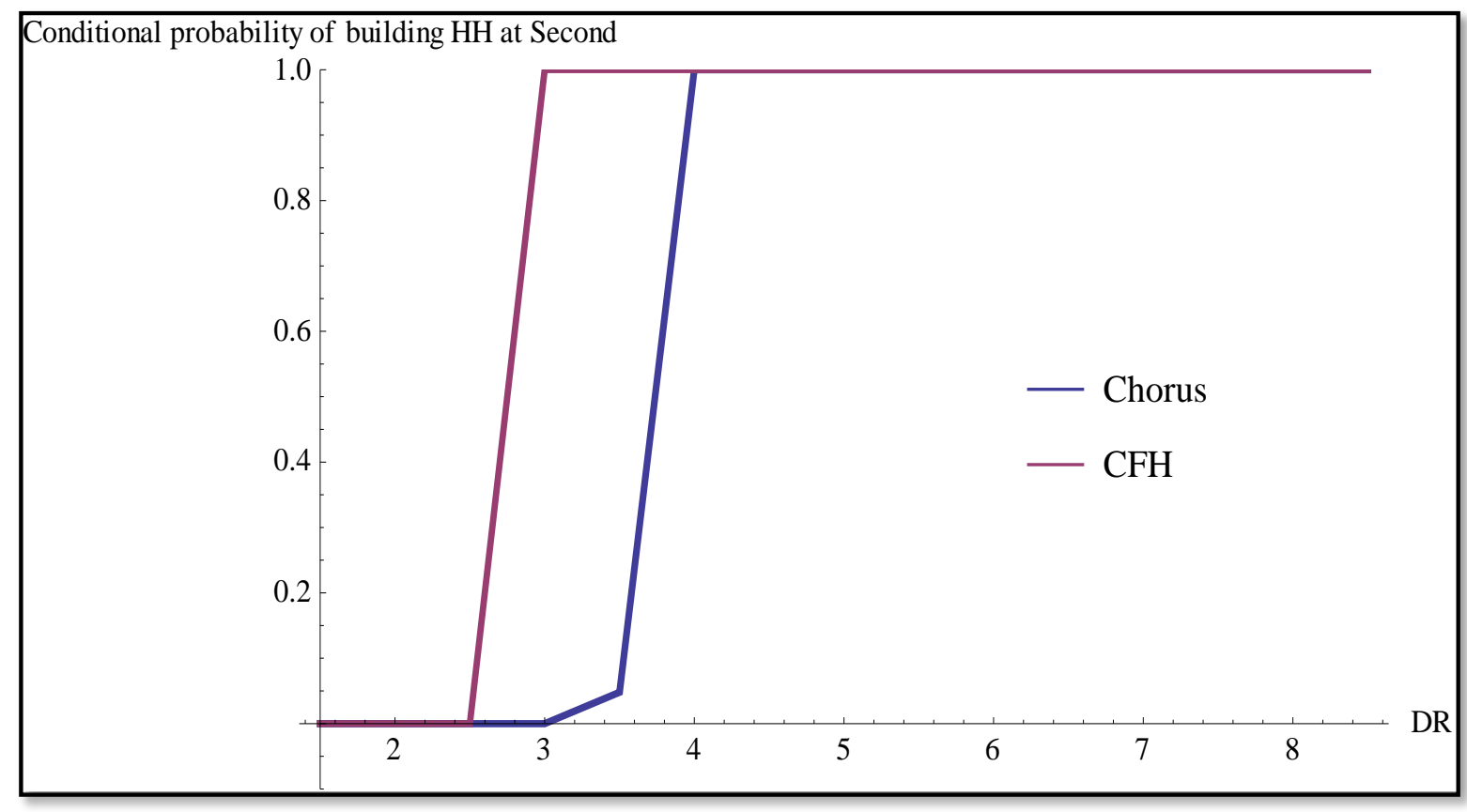

In contrast, controlling for $\boldsymbol{D R}$ at graph 6.10, both parties agree to finish city HH before city LL when the cost multiplier is smaller than 1.6 (the conditional probabilities of building city HH second are equal to one for both parties at graph 6.10). As for the case of the demand multiplier, we find a sequencing conflict when the cost multiplier ranges between 1.6 and 2.4. Further on, when the cost multiplier goes over 2.4 , the sequencing conflict vanishes and both parties agree to finish the city LL before city HH. For both $\boldsymbol{D R}$ and $\boldsymbol{C R}$, since the sequencing conflict only shows up in a range of parameters, it cannot be the major factor to drive the conflict level.

The intuition behind the shift of construction preference is clear. As we learnt from the previous setting, city HL always dominates the other three cities, while city LH is always dominated by the others. Therefore, Chorus and CFH always order city HL first and city LH last. The only potential disagreement between these two parties on ordering will be city $\mathrm{HH}$ and city LL. Providing that a constant cost multiplier holds, when we increase the demand multiplier alone, the rising revenues make city $\mathrm{HH}$ relatively more profitable than city LL. Therefore both parties start to shift their construction preferences from city LL to city HH. However, when the $\boldsymbol{D} \boldsymbol{R}$ goes up, CFH reaches the margin that city $\mathrm{HH}$ will be preferred over city LL earlier than Chorus due to the extra consumer surplus, which explains why the preference of $\mathrm{CFH}$ shifts faster than that of Chorus at graph 6.9. 


\section{Graph 6.10: The sequencing conflict measure varies over $C R$}

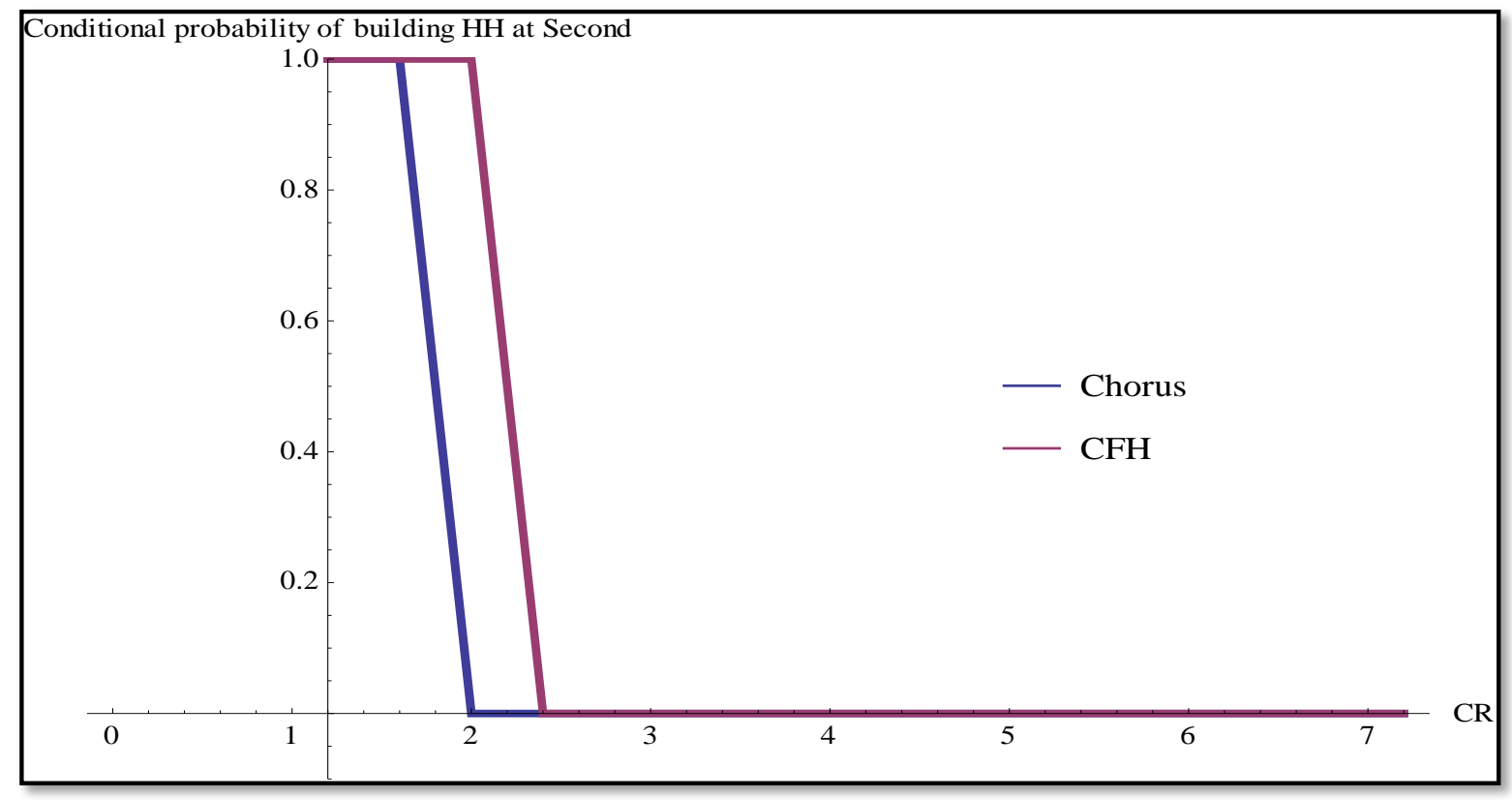

On the other hand, when we increase the cost multiplier alone, the rising capital expenditures make city HH relatively more expensive (less profitable) than city LL. Therefore both parties start to prefer city LL over city HH. Thanks to the extra consumer surplus, CFH reaches the margin that city LL will be preferred over city HH later than Chorus when $\boldsymbol{C R}$ increases, which explains why the preference of $\mathrm{CFH}$ shifts slower than that of Chorus at graph 6.10.

At graph 6.11, we plot the timing conflict measure as a function of $\boldsymbol{D R}$. Here, the expected number of stages completed in 4 periods gives us the idea about the timing differences of the highly profitable cities between CFH and Chorus (which are city HL and city HH (when $\boldsymbol{D R}$ is relatively high)), while the expected number of stages completed in 24 periods adds up the timing information about the marginal cities (which are city LL and city HH (when $\boldsymbol{D R}$ is relatively low) $).^{70}$ When we raise $\boldsymbol{D R}$, the increase in the demand ratio raises both the producer surplus and consumer surplus of the high demand cities (which are city HL and city $\mathrm{HH}$ ), which definitely makes the high demand cities more preferable to both parties. On the one hand, the increases of surplus flows from raising $\boldsymbol{D R}$ have no impact on the two parties'

\footnotetext{
${ }^{70}$ We group city $\mathrm{HL}$ and city HH (when DR is big) into the profitable cities, and city LL and city HH (when DR is small) into the marginal cities. For example, when DR equals 6 , we find the present values of total surplus for both city $\mathrm{HL}$ and city $\mathrm{HH}$ are over 100 , while the corresponding present value for city LL is only 2 . In contrast, when we set DR equal to 1.5 , the present value of total surplus for city $\mathrm{HH}$ is 0.25 .
} 
timing disagreement on city HL. ${ }^{71}$ On the other hand, the increases of surplus flows from raising $\boldsymbol{D R}$ either aggravate or reduce the two parties' timing disagreement on city $\mathrm{HH}^{72}$ In summary, the increase of $\boldsymbol{D R}$ has an ambiguous effect on the timing conflict.

\section{Graph 6.11: The timing conflict measure varies with $D R$}

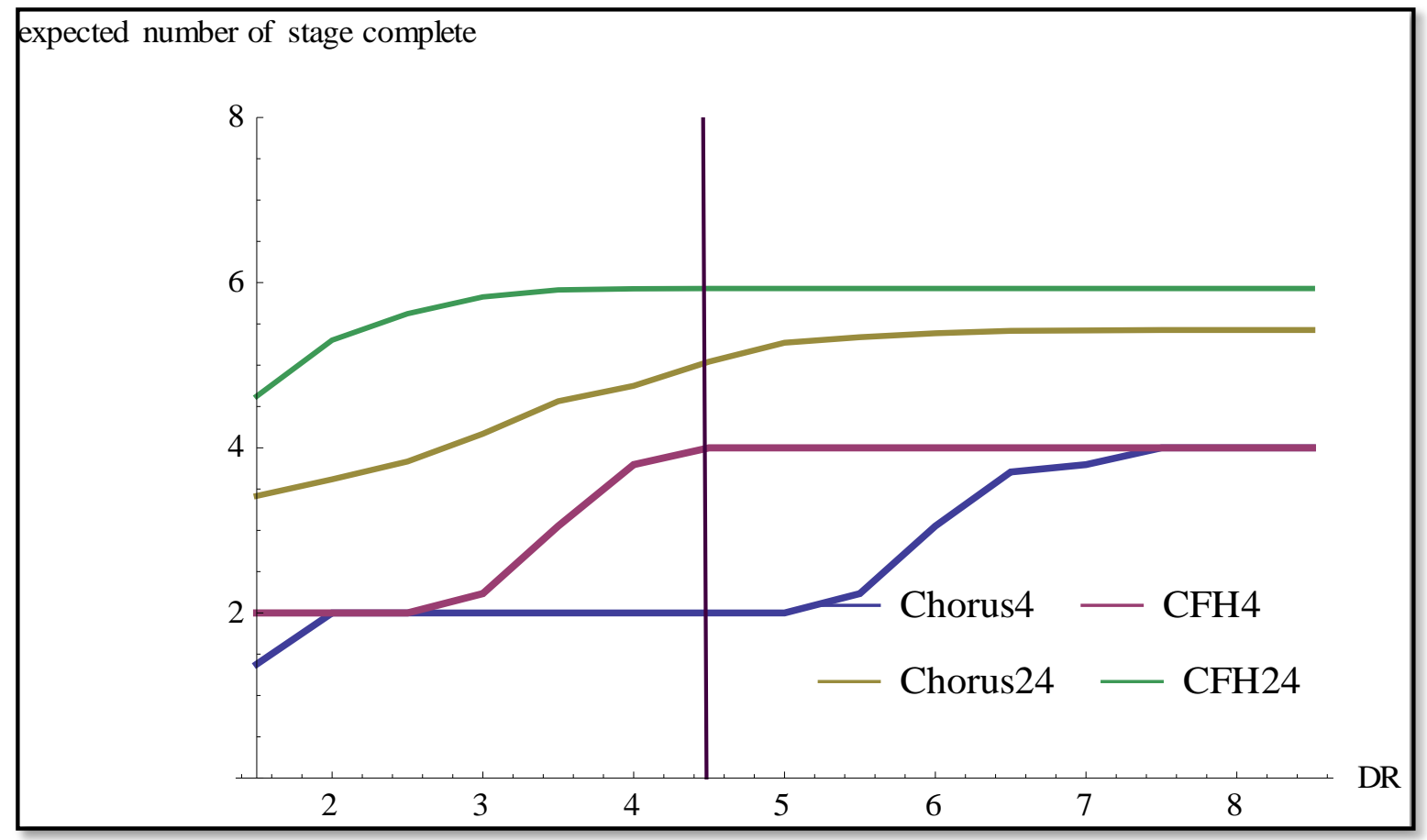

When we increase $\boldsymbol{D} \boldsymbol{R}$, city HH shifts from a marginal city to a profitable city. Therefore, in order to understand the movement of the timing conflict on city $\mathrm{HH}$, we have to combine the expected numbers of stages completed in both 4 periods (which is a good timing measure for a profitable city) and 24 periods (which is a good timing measure for ta marginal city). At graph 6.11, when we raise $\boldsymbol{D} \boldsymbol{R}$ from 1.5 to 3, we observe an increase of the timing conflict on city $\mathrm{HH}$ (see the increased difference of the expected number between two parties for 24

\footnotetext{
${ }^{71}$ Since city $\mathrm{HL}$ is so profitable (for all selective DR) that both parties want to build it up as fast as they can, there is no timing conflict between $\mathrm{CFH}$ and Chorus on city $\mathrm{HL}$ regardless.

72 The effect is attributed to the extra consumer surplus flow of CFH. That is, compared to Chorus, the existence of extra consumer surplus means that $\mathrm{CFH}$ requires a relatively small DR in order to want to complete city $\mathrm{HH}$. Therefore, if we raise DR when it is relatively small, CFH may start to finish city $\mathrm{HH}$ eventually, while Chorus considers city HH is still worthless, which we observe as an increase of timing conflict on city $\mathrm{HH}$ between two parties. On the other hand, if we raise DR when it is relatively large, it may start to improve Chorus's timing of city $\mathrm{HH}$ but have no effect on CFH's timing of city HH (Since CFH already decides to complete city $\mathrm{HH}$ as soon as possible). This is why we observe a reduction of timing disagreement on city $\mathrm{HH}$ between two parties.
} 
periods). ${ }^{73}$ When $\boldsymbol{D R}$ is between 3 and 4.5 , we find that the timing conflict on city $\mathrm{HH}$ increases over $\boldsymbol{D R}$ (see the increased difference in the expected number of stages completed in 4 periods). Since the timing conflict on city $\mathrm{HH}$ dominates the timing conflict (in the aggregated level), we expect the timing conflict increases if we raise $\boldsymbol{D R}$ when it is relatively small ( $\boldsymbol{D} \boldsymbol{R} \leq 4.5)$. After reaching the maximum, the timing conflict on city HH starts to decrease over $\boldsymbol{D R}$ (see the decreased difference in the expected number of stages completed in 4 periods when $4.5 \leq \boldsymbol{D R} \leq 7.5$ ). Further on, when we raise $\boldsymbol{D} \boldsymbol{R}$ over 7.5 , both parties would like to finish city $\mathrm{HH}$ in 4 periods therefore the timing conflict on city $\mathrm{HH}$ disappears $^{74}$ (the difference of the expected number for 4 periods is zero). The only timing conflict left is for city LL, ${ }^{75}$ which remains constant over $\boldsymbol{D R}$ (the difference of the expected number for 24 periods is constant when $7.5 \leq \boldsymbol{D R})$. In summary, we expect the timing conflict decreases if we raise $\boldsymbol{D R}$ when it is relatively large $(4.5 \leq \boldsymbol{D R})$.

\section{Graph 6.12: The timing conflict measures varies with $C R$}

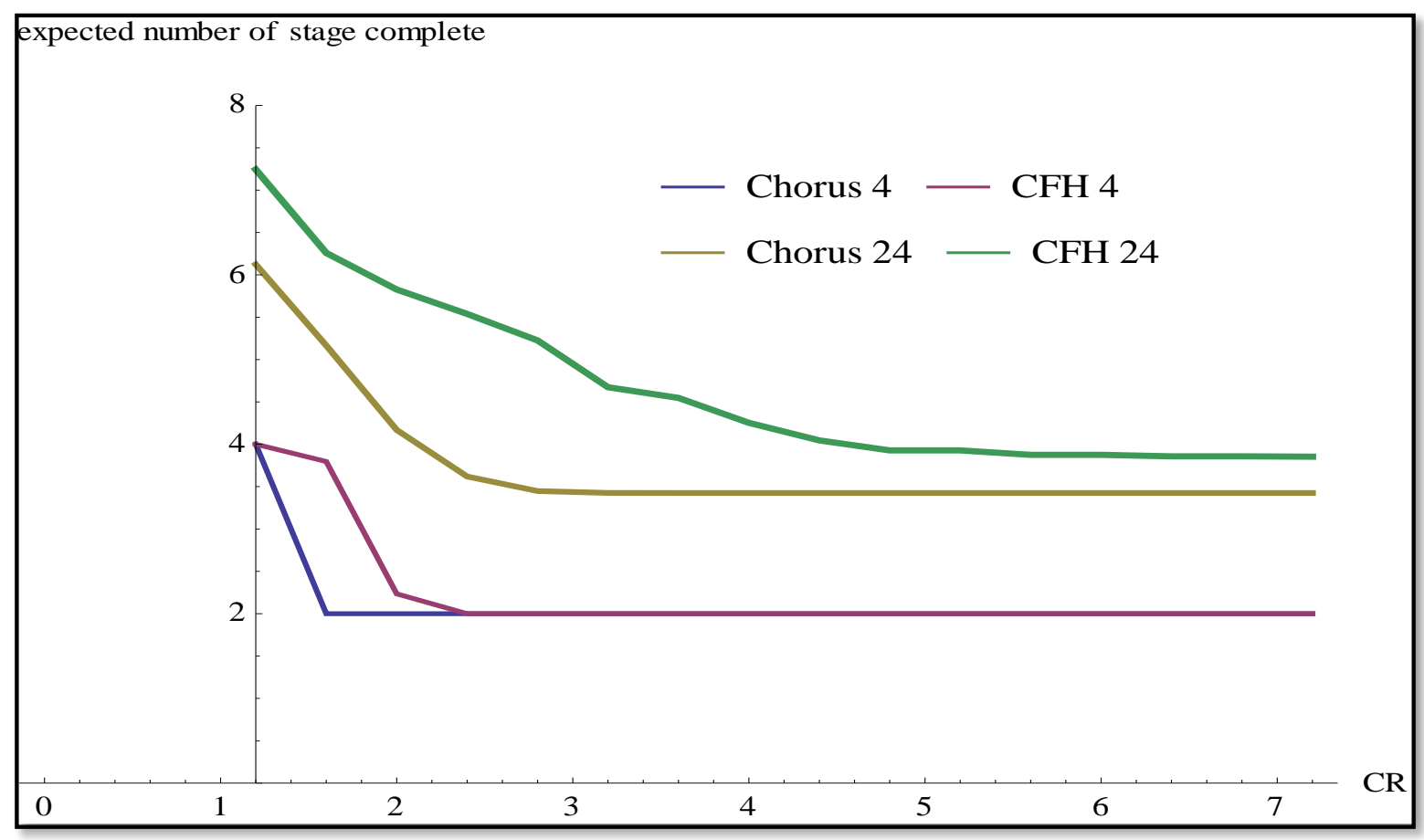

\footnotetext{
${ }^{73}$ Although the construction of city $\mathrm{HH}$ is becoming more and more attractive to both parties when we raise $D R$, it is still not profitable enough to induce either player to finish it within 4 periods. Hence, the timing measure for 4 periods is not sensitive to the movement of DR.

${ }^{74}$ For both CFH and Chorus, it is the fastest speed they can have to build up city HH unless they can exercise the speed up option. However, it is unreasonable to do it at the first few nodes since the improvement of state is never high enough to justify the cost of exercising speed up option.

${ }^{75}$ Although the timing conflict on city LL exists when DR $\leq 7.5$, it is dominated by the timing conflict on city $\mathrm{HH}$.
} 
Due to the increased capital expenditure, city $\mathrm{HH}$ will soon became a marginal city when we raise $\boldsymbol{C} \boldsymbol{R}$. Hence, the expected number of construction stages completed in 24 periods (which is a good timing measure for a marginal city) may provide us more information about the movement of timing conflict on city $\mathrm{HH}$. Since the timing conflict on city $\mathrm{HH}$ dominates the aggregate timing conflict, the movement of timing conflict on city $\mathrm{HH}$ indicates the movement of aggregate timing conflict over $\boldsymbol{C R}$.

At graph 6.12, when we raise $\boldsymbol{C} \boldsymbol{R}$ from 1.2 to 2.4, we find the difference on the expected number of construction stages completed in 24 periods between two parties increases from 1.1 to 1.9. As soon as it reaches the peak, the timing conflict on city $\mathrm{HH}$ starts to decrease over $\boldsymbol{C} \boldsymbol{R}(\boldsymbol{C R} \geq 2.4)$. When we compare graph 6.12 with graph 6.8 , we find the movements of timing conflict on city $\mathrm{HH}$ and the present value of conflict (in the aggregate level) are quite similar (that is, the increased capital expenditure from raising $\boldsymbol{C} \boldsymbol{R}$ has an ambiguous effect (non-monotonic) on both the timing conflict on city $\mathrm{HH}$ and the present value of the aggregate timing conflict ${ }^{76}$ ). The only difference we find is that they peak at different levels of $\boldsymbol{C R}$. That is, the timing conflict measure on city HH peaks when $\boldsymbol{C R}$ equals 2.4, while the present value of aggregate timing conflict peaks when $\boldsymbol{C R}$ equals 2 . However, this distortion results from the fact that the decrease in magnitude of the present value of total surplus on building up city $\mathrm{HH}$ (which is caused by the increase of $\boldsymbol{C R}$ ) dominates the increase of timing conflict on city HH. That is, when we increase $\boldsymbol{C R}$ from 2 to 2.4 , we find that the present value of total surplus from constructing city HH under the two parties' policies decreases from 8.4 to 3.6 (more than half), while the corresponding timing conflict only increases from 1.7 to 1.9 . Recall that, the timing conflict on city $\mathrm{HH}$ is a good proxy for the aggregate timing conflict. Therefore, the above similarity shows us that the present value of conflict could be well explained by the timing conflict.

\footnotetext{
${ }^{76}$ Compared to Chorus, the existence of extra consumer surplus slows down CFH's speed in abandoning city $\mathrm{HH}$. Therefore, if we raise $\mathrm{CR}$ when it is relatively small, Chorus may change its construction preference on city $\mathrm{HH}$ faster than that of $\mathrm{CFH}$, leading to an increase of timing conflict on city $\mathrm{HH}$ between two parties. On the other hand, if we raise CR when it is relatively large, it may still be able to change CFH's timing of city HH but have no effect on Chorus' timing of city $\mathrm{HH}$ (Since Chorus already abandons city HH completely), leading to a reduction of timing disagreement on city $\mathrm{HH}$ between the two parties.
} 


\section{Chapter 7: The optimal incentive tools}

In this section, we are going to study the efficiencies of several incentive vehicles in reducing the possible conflicts. More specifically, we not only investigate what will be the minimum conflict level for each incentive tool, but also how the incentive scheme reduces the relevant conflicts. Further on, we compare these incentive schemes in their abilities of reducing the possible conflicts.

\subsection{The study of subsidy schemes}

\subsubsection{The optimal proportional subsidy}

Recall that the proportional subsidy is a monetary transfer from $\mathrm{CFH}$ to Chorus, which is proportional to Chorus' current capital expenditure level. The proportional subsidy scheme requires the public party being able to learn about Chorus's "actual" capital expenditure at each period. In order to identify the global minimum conflict level, we first vary the proportional subsidy with a small step ( 0.01 for per step), which causes the graph of the present value of conflict to become quite lumpy ${ }^{77}$. Therefore, to reconstruct a relatively smooth curve but guarantee the global minimum conflict level, we extract the turning point (the minimum) from the lumpy curve and plot it at graph 7.1. Starting from this turning point, we plot the conflict level corresponding to a bigger step increase in the proportional subsidy ( 0.05 for per step). That is, beginning from 0.41 subsidy rate, we plot the corresponding conflict level either when we decrease the subsidy rate by 0.05 on the left hand side or increase the subsidy rate by 0.05 on the right hand side. We report a relatively smooth curve for how the conflict level varies with the proportional subsidy at graph 7.1.

\footnotetext{
${ }^{77}$ The lumpiness of the curve is due to the binomial tree method. That is, a small change in the subsidy rate results in a small change in all the values of cash flows (In the binomial tree) and therefore a small change in Chorus's construction behavior in reality. However, with the discrete method we apply, there can be a relatively large change in our binominal tree. For example, it may require Chorus to have 1.9 up moves to construct city $\mathrm{HH}$ in reality. After a small change to the subsidy rate, Chorus needs to have 2.1 up moves to build up city $\mathrm{HH}$. As a result, in the discrete method, instead of constructing city HH with 2 up moves, Chorus then only exercises city $\mathrm{HH}$ with three up moves despite there being only a small change in the subsidy rate.
} 


\section{Graph 7.1: The present value of conflict varies with proportional subsidy}

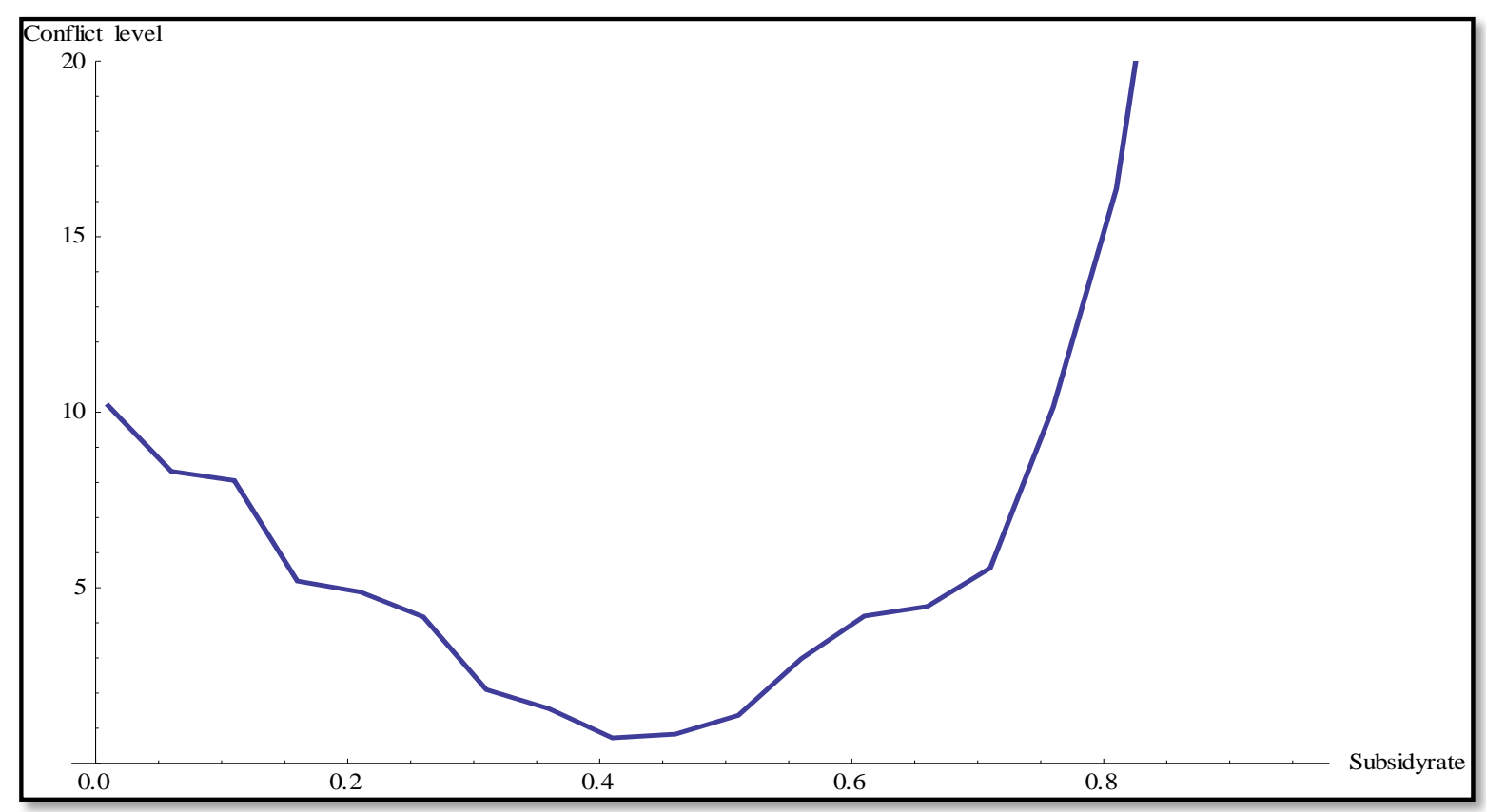

Clearly, the conflict level first decreases and then increases with the proportional subsidy rate. The conflict between the two parties reaches the minimum level $(0.72)$ when the subsidy rate equals 0.41 . That is, at each period, the optimal monetary transfer from $\mathrm{CFH}$ to Chorus should equal 41 per cent of the private parties' capital expenditure in that period. The logic behind the non-monotonic change of the conflict level is quite straightforward. Chorus becomes more aggressive in the UFB network's construction when there is a reduction in the proportion of capital expenditure that Chorus needs to bear. Therefore, the decrease of the conflict level at first is due to reducing the difference between $\mathrm{CFH}$ and Chorus in their construction preferences. ${ }^{78}$ In contrast, in the second half of the graph, the further increase of the subsidy rate makes Chorus more aggressive than CFH in the UFB network's construction. Therefore the deviation of construction preferences between the two parties starts to increase. Consequently, we observe an increase of the conflict level with the subsidy rate in the second half of the graph.

\footnotetext{
${ }^{78}$ When there is no subsidy, Chorus is less aggressive than CFH in the UFB network's construction. The add-on of a subsidy increases Chorus's aggression in construction therefore resulting in a better match between the two parties' construction preferences.
} 


\section{Graph 7.2: The present values to CFH of the individual cities (under Chorus's optimal policy) vary with proportional subsidy}

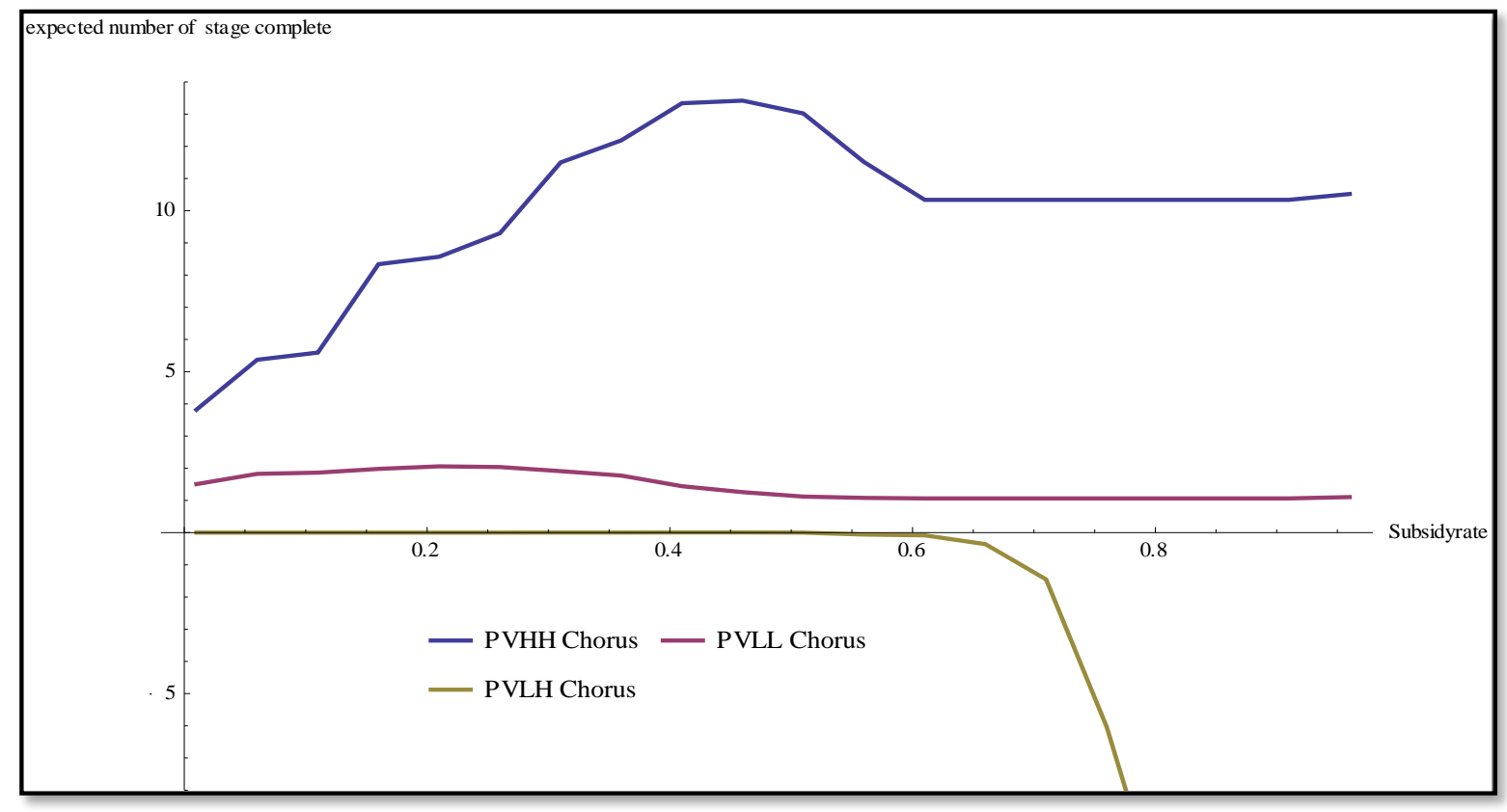

The present value to $\mathrm{CFH}$ of constructing an individual city (under Chorus' optimal policy) may further support the non-monotonic change of conflict level, and most important, explain why its minimum lies at the 0.41 subsidy rate. Graph 7.2 plots the present values to $\mathrm{CFH}$ of the individual cities. ${ }^{79}$ At graph 7.2, the present value of city $\mathrm{HH}$ increases with the proportional subsidy and peaks at a subsidy rate of about 0.45 , while the present value of city LL has its peak at a subsidy rate of 0.2 and then slowly decreases with the proportional subsidy. The minimum conflict level, of course, balances the marginal increase of the present value of city $\mathrm{HH}$ and the marginal decrease of the present value of city LL. That is, the marginal increase of PV HH dominates the marginal effects of PV LL and PV LH when the subsidy rate is smaller than 0.41 , while the corresponding increase of PV HH is in turn dominated by the decrease of PV LL when the subsidy rate ranges from 0.41 to 0.45 . Therefore, the minimum conflict level occurs at a subsidy rate of 0.41 . Besides, the sharp diving of PV LL explains why the conflict level rockets up after the proportional subsidy rate goes over 0.7 (The present value of LH starts to dominate the conflict level when the proportional subsidy rate reaches 0.7 ). To see why this happens, recall that the objective

\footnotetext{
${ }^{79}$ We do not plot the city $\mathrm{HL}$ due to two reasons. 1) The present value of city $\mathrm{HL}$ is so high that it would distort the graph (compared with the other three cities). 2) The present value of city HL remains independent of the subsidy rate since the subsidy schemes have no impact on Chorus' construction behaviors on city $\mathrm{HL}$ (City $\mathrm{HL}$ is so profitable that Chorus will construct it as fast as CFH).
} 
function of $\mathrm{CFH}$ is to maximize the difference between the present value of the sum of producer surplus and consumer surplus and the capital expenditure, while the objective function of Chorus is to maximize the difference between the present value of the sum of producer surplus and subsidy and the capital expenditure. If the value of the subsidy is far bigger than the consumer surplus of city LH, Chorus may still build up city LH very fast to get the subsidy when the state of demand is not favourable. However, CFH may choose to abandon the construction of city LH when the state of demand is not favourable, since the corresponding consumer surplus is not sufficient to justify the related construction costs. That is, when the proportional subsidy is high, Chorus would be over-subsidized to construct city LH very fast, which deviates from CFH's optimal policy. Therefore, the present value to CFH of city LH drops dramatically when the subsidy rate is high.

Now, we incorporate both timing conflict and sequencing conflict measures into our discussion. We focus more on the timing conflict measure rather than the sequencing conflict measure since the present value of conflict is mainly attributed to the two parties' differences in construction timing.

\section{Graph 7.3: The timing conflict measures vary over proportional subsidy}

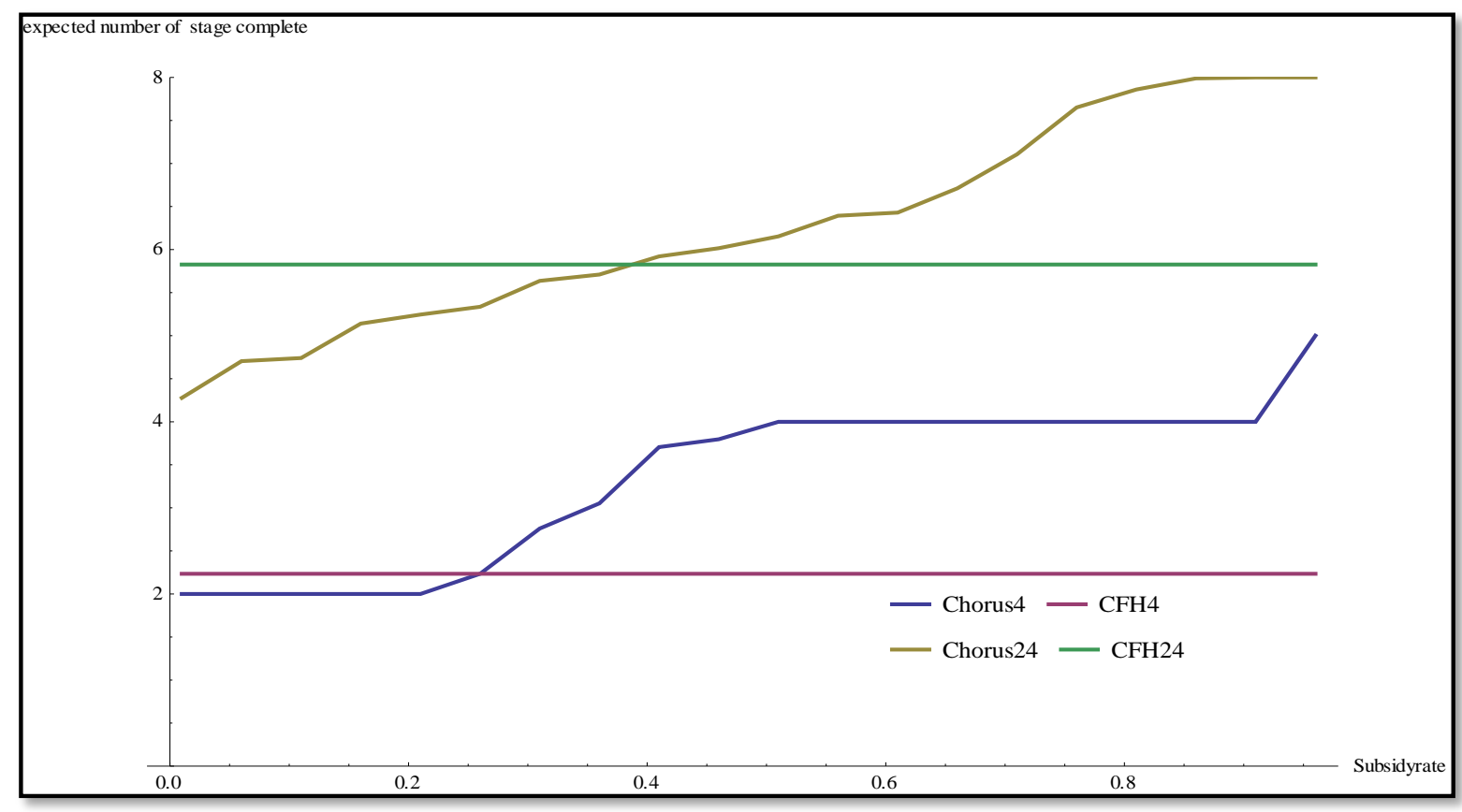

For the timing conflict measure, we provide the expected numbers of construction stages completed in both 4 and 24 periods at graph 7.3. Unsurprisingly, the expected numbers of 
construction stages completed for CFH remain constant over the proportional subsidy ${ }^{80}$ (which is the red line and the green line at graph 7.3). For Chorus, the expected numbers of construction stages completed are smaller than that of $\mathrm{CFH}$ when there is a small subsidy.(This is consistent with the fact that Chorus is less aggressive than $\mathrm{CFH}$ in the network's construction when there is no subsidy) However, when we start to increase the proportional subsidy, we find that the expected numbers of construction stages completed for Chorus are increasing (which is the yellow line and the blue line at graph 7.3). That is, the proportional subsidy scheme generally accelerates Chorus' timing in network's construction. $^{81}$

We find that the timing conflict in 4 periods (which is the difference in the expected number of construction stages completed in 4 periods at graph 7.3) follows a similar non-monotonic change as the present value of city LL, while the timing conflict in 24 periods (which is the difference in the expected number of construction stages completed in 24 periods at graph 7.3) follows a similar non-monotonic change as the present value of city $\mathrm{HH}^{82}$ In other words, the graph 7.3 supports the interpretation of graph 7.1 .

As we observe from graph 7.4, the conditional probability that $\mathrm{CFH}$ wants to complete city $\mathrm{HH}$ second providing that at least two cities will be finished always equals 1 (Since the subsidy does not affect CFH's construction behaviour), while the corresponding conditional probability for Chorus monotonically increases over the proportional subsidy. Therefore, the scale of the sequencing conflict is monotonic decreasing over the proportional subsidy, and entirely disappears when the proportion of subsidy goes over 56 per cent. Namely, both parties agree to finish the city HH before city LL after the proportional subsidy rate reaches the certain level. ${ }^{83}$

\footnotetext{
${ }^{80}$ Recall that CFH's construction behaviors are not affected by the subsidy scheme.

${ }^{81}$ It is intuitive that the less capital expenditure Chorus needs to bear the faster it wants to build up the UFB network.

${ }^{82} \mathrm{PV}$ LL and the timing conflict measure in 4 periods peak at about 0.2 subsidy rate, while PV HH and the timing conflict measure in 24 periods peak at around 0.4 . Recall that the timing conflict measure in 4 periods is a good proxy for the profitable city's timing (which is city LL for Chorus), while the timing conflict measure in 24 periods is a good proxy for the marginal city's timing (which is city HH for Chorus).

${ }^{83}$ The logic behind Chorus' shift of construction preference is clear. When there is no subsidy, Chorus may prefer city LL over city $\mathrm{HH}$. However, for any one per cent increase in the proportional subsidy, city $\mathrm{HH}$ receives more compensation in dollar term than city LL. As a result, when we keep increasing the subsidy rate, the relative profitability of city LL to city $\mathrm{HH}$ will decrease over time. Therefore, Chorus may start to prefer city $\mathrm{HH}$ when the subsidy rate reaches the certain level.
} 


\section{Graph 7.4: The sequencing conflict measures vary over proportional subsidy}

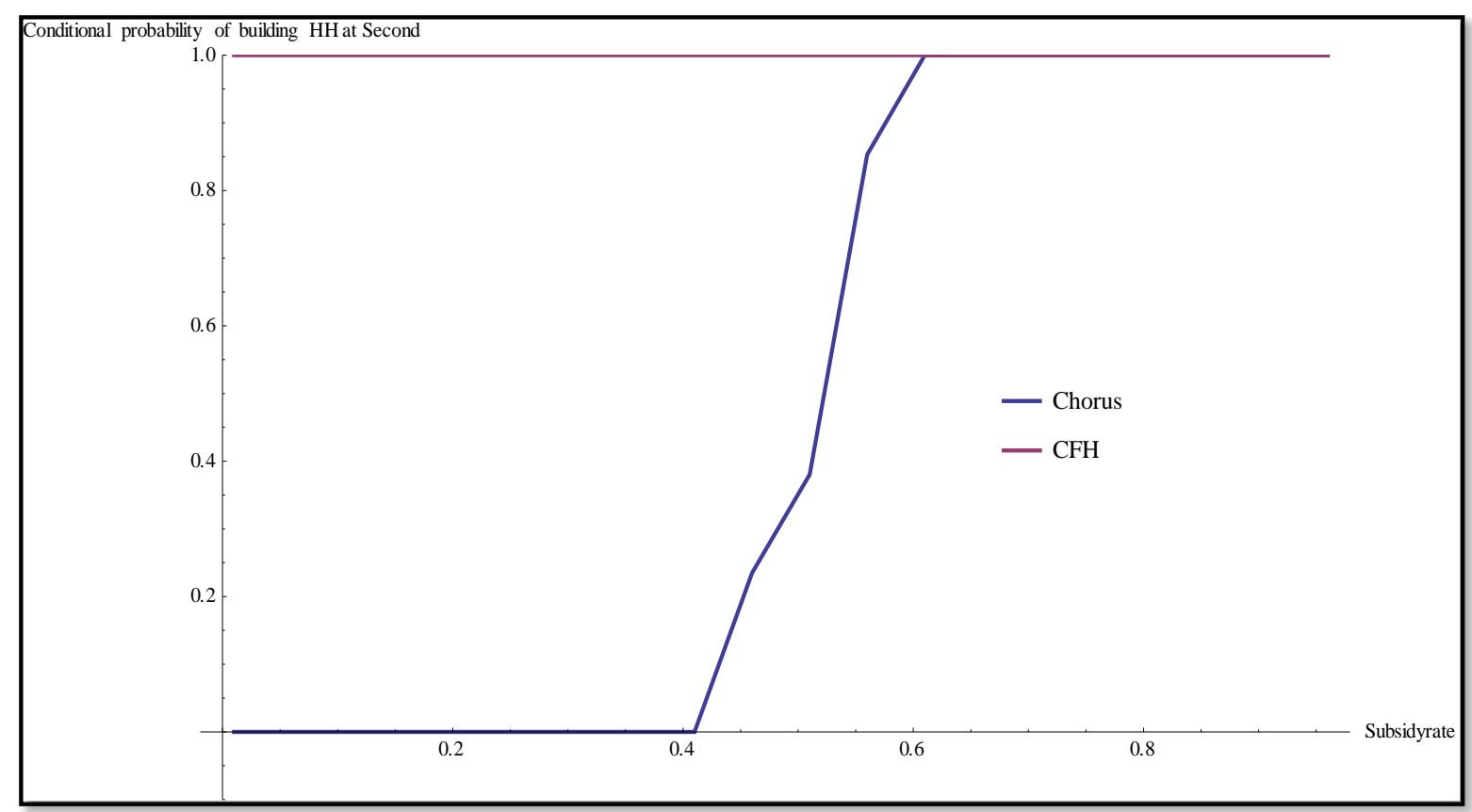

\subsubsection{The optimal fixed subsidy}

Like the proportional subsidy in the previous section, the fixed subsidy is a monetary transfer from $\mathrm{CFH}$ to Chorus. However, instead of being proportional to Chorus's current capital expenditure level, the fixed subsidy scheme requires $\mathrm{CFH}$ to make a fixed payment to Chorus for undertaking each construction stage in a period. Namely, the fixed subsidy scheme requires the public party being able to learn about how many construction stages in total Chorus are undertaking each period but not whether construction is high or low cost. We report how the conflict level varies with the level of the fixed subsidy at graph 7.5.

As for the proportional subsidy scheme, the conflict level first decreases and then increases with the level of fixed subsidy. Nevertheless, the minimum conflict level resulting from the fixed subsidy scheme equals 0.95 , which is higher than the minimum conflict level (0.72) resulting from the proportional subsidy scheme. The corresponding optimal fixed subsidy level equals 8.95 .

The reason behind the non-monotonic change of conflict level over the fixed subsidy is exactly the same as what we find from the proportional subsidy scheme. One strong support for this argument is that, for both proportional and fixed subsidy schemes, Chorus's expected 
numbers of construction stages completed (the measure of construction timing) are increasing with subsidies. ${ }^{84}$

\section{Graph 7.5: The present value of conflict varies with fixed subsidy}

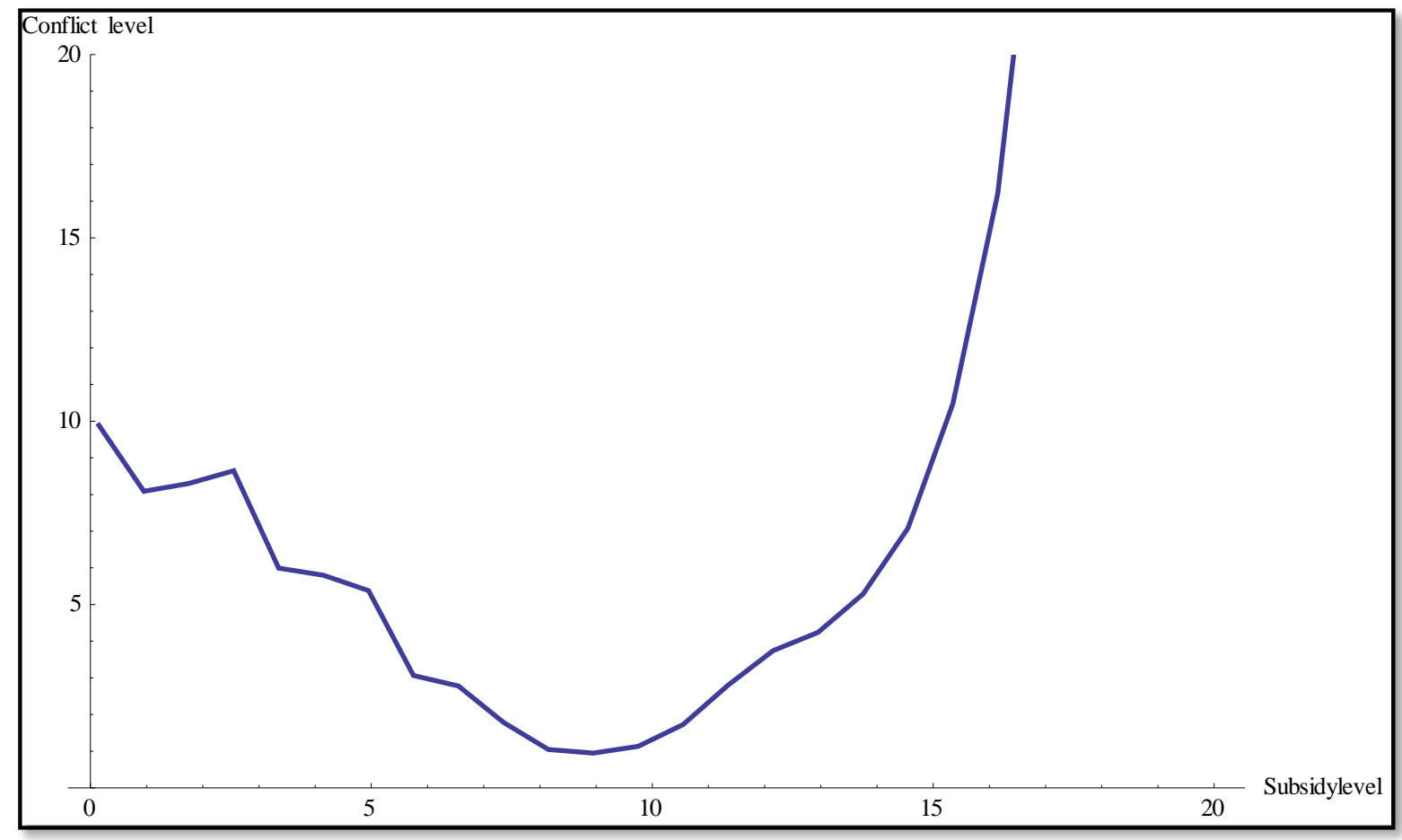

Similarly, we apply the present values to CFH of individual cities (under Chorus' optimal policy) to understand the minimum level of conflict under the fixed subsidy. At graph 7.6, the present value of city $\mathrm{HH}$ grows over the fixed subsidy and has a peak when the fixed subsidy sits about 8.9 . On the other hand, the present value of city LL reaches the maximum when the subsidy level equals 1 . After dropping down from the peak, the present value of city LL remains constant for high levels of the fixed subsidy. Since the movement of PV HH dominates when the fixed subsidy is not too high, the minimum conflict level, of course, should be a project of PV HH. Therefore, the minimum conflict level occurs at a subsidy level of 8.9. As for the proportional subsidy, the huge jump of conflict level is attributed to over-investment in city LH when the level of the fixed subsidy is very high. ${ }^{85}$

\footnotetext{
${ }^{84}$ This implies that Chorus is becoming more and more aggressive in the UFB network's construction when we increase the level of fixed subsidy.

${ }^{85}$ As for the proportional subsidy, the high level of fixed subsidy encourages Chorus to build up city LH very quickly, which is not the optimal behavior to CFH. Therefore, the PV LH drops dramatically when the subsidy rate is high.
} 


\section{Graph 7.6: The present values to CFH of the individual cities (under Chorus' optimal policy) vary with fixed subsidy}

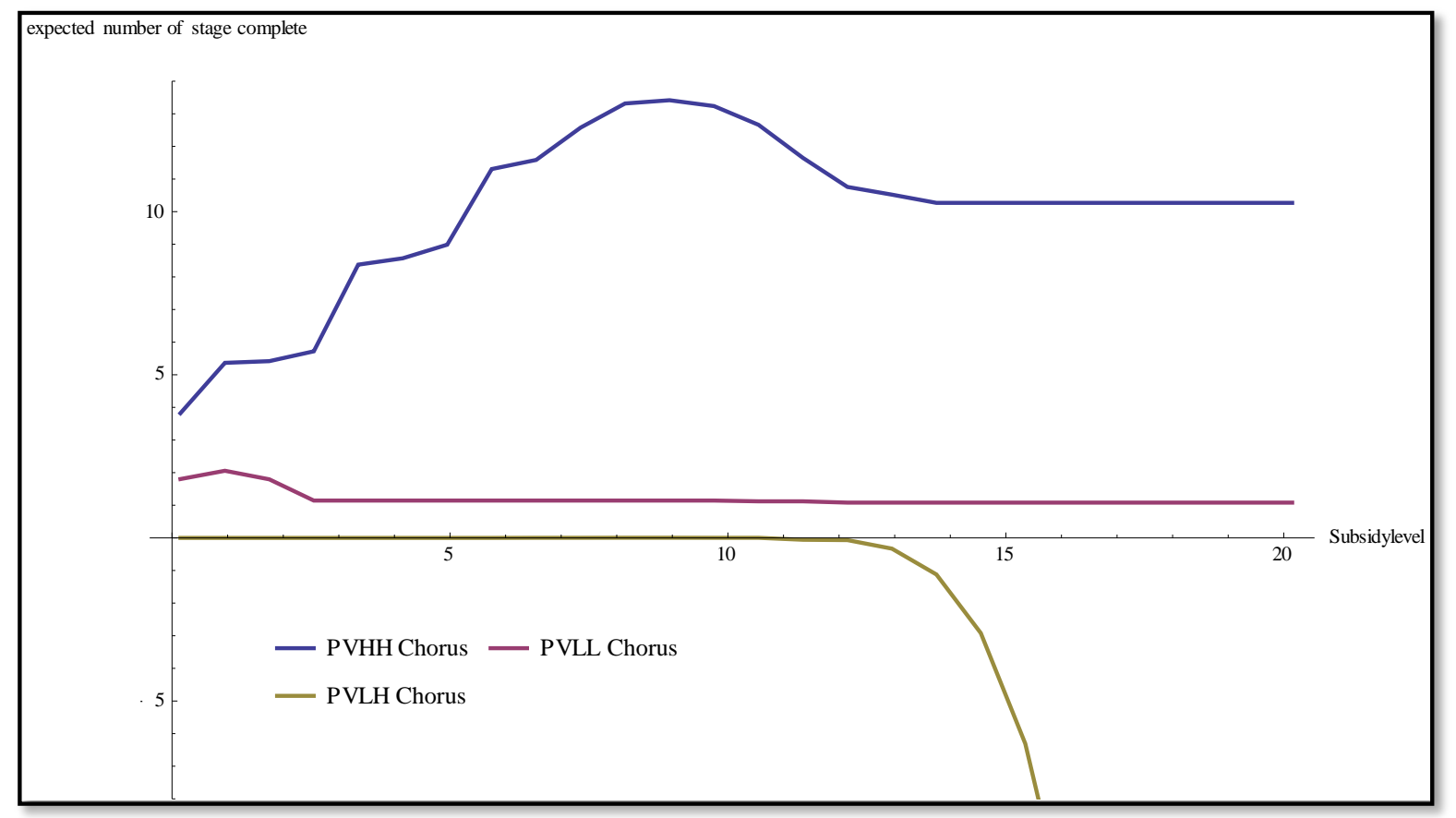

For the fixed subsidy, we find the corresponding timing measures share the same features as that of the proportional subsidy. That is, A) the fixed subsidy scheme generally accelerates Chorus's timing in the network's construction; B) the timing conflict measures follow the same non-monotonic paths as the present values of city HH and city LL. ${ }^{86}$

We find the scale of the sequencing conflict decreases over the level of fixed subsidy, which is similar to that under the proportional subsidy scheme. However, the fixed subsidy scheme never makes the sequencing conflict disappear entirely (the conditional probability that Chorus wants to complete city $\mathrm{HH}$ second providing that at least two cities will be finished never reaches 1$)$.

\footnotetext{
${ }^{86}$ The slight mismatch between the timing conflict in 24 periods and $\mathrm{PV} \mathrm{HH}$ may result from the fact that, although the timing conflict in 24 periods is a good proxy for the timing of city $\mathrm{HH}$, it is distorted by the timing of city LL (as an aggregate timing measure). As we can see, Chorus becomes more aggressive than CFH in building up city LL when the subsidy level is low.
} 
Graph 7.7: The timing conflict measures vary over the fixed subsidy

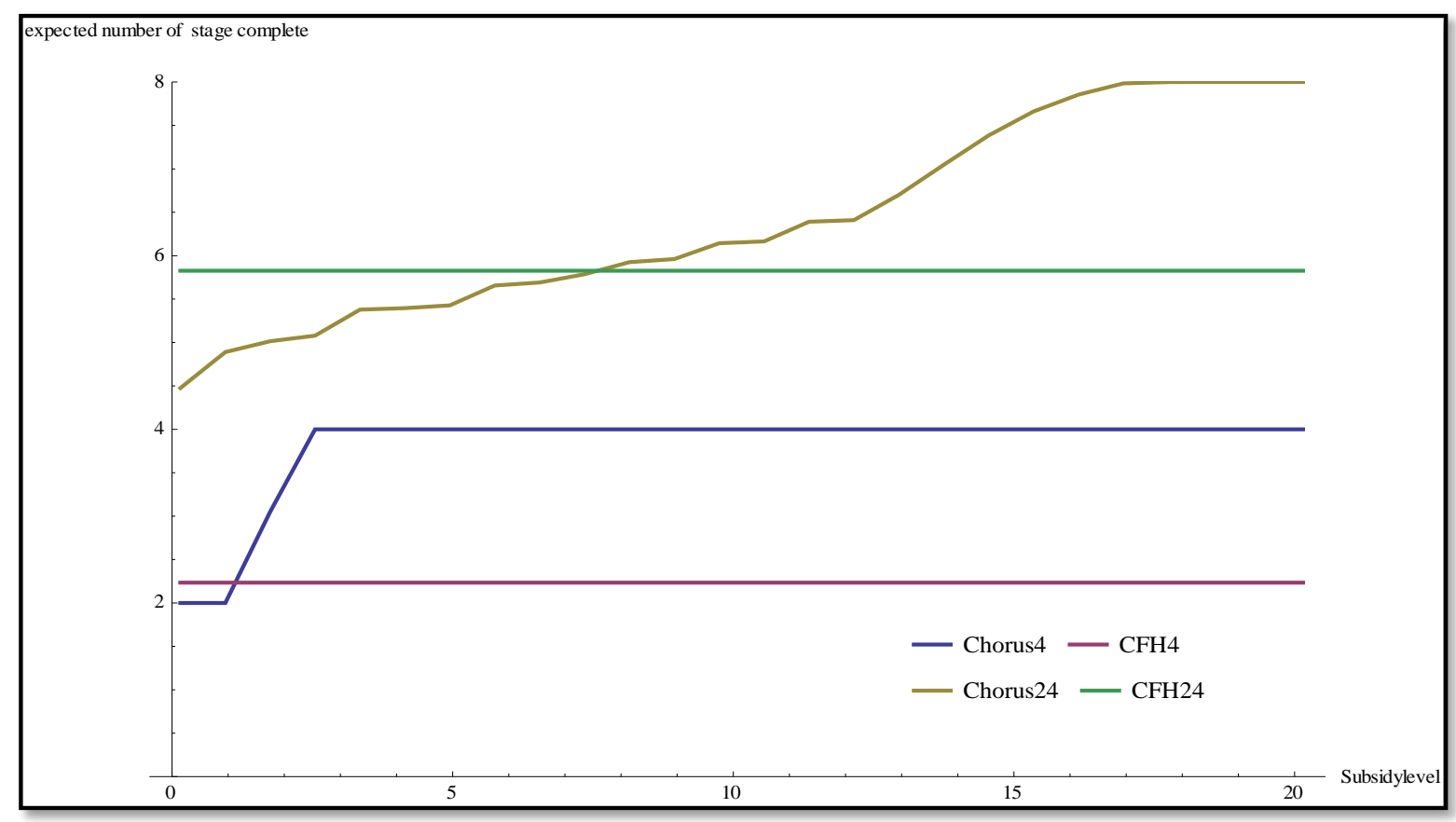

Graph 7.8: The sequencing conflict measures vary over fixed subsidy

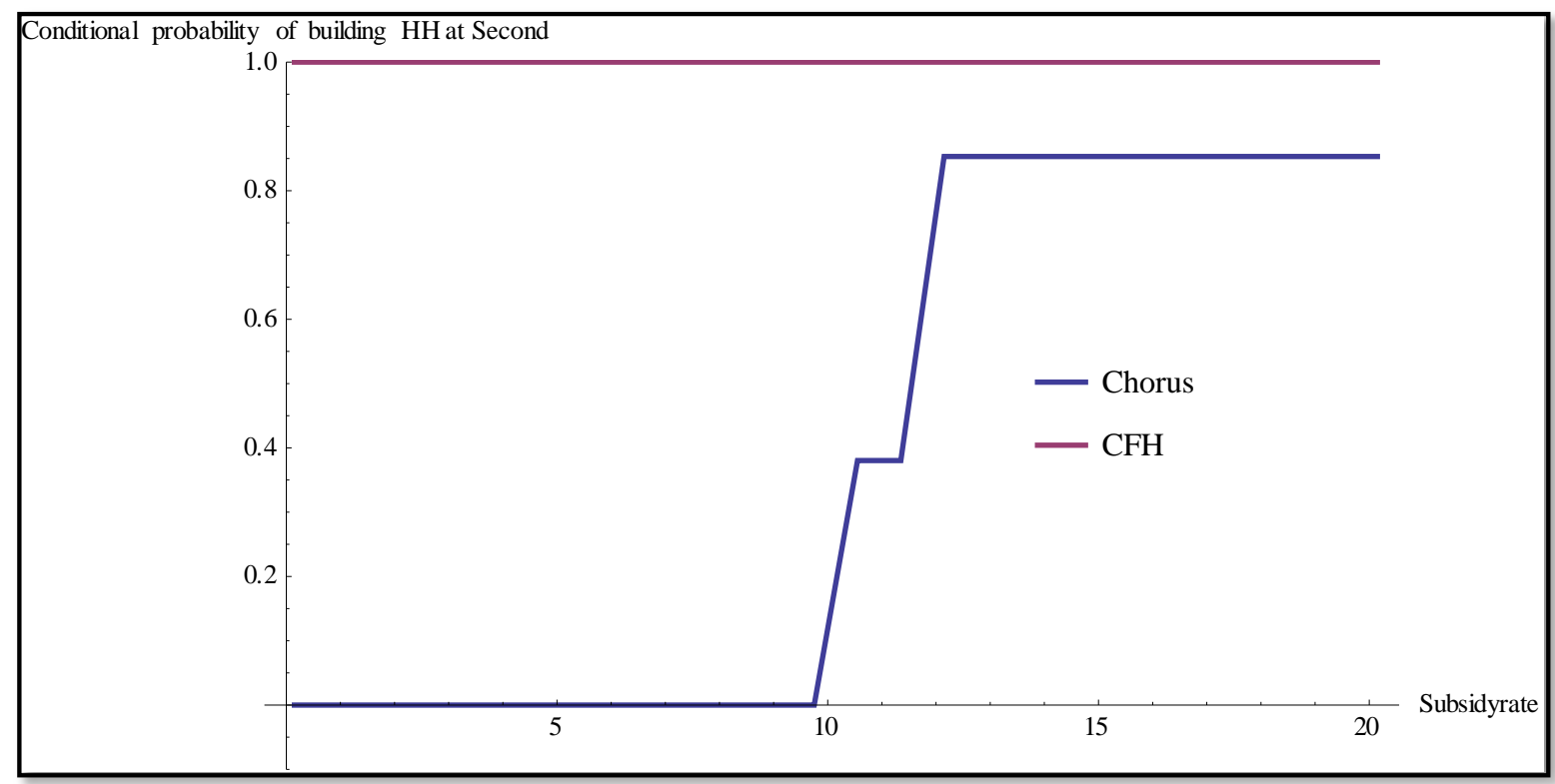

\subsubsection{The optimal lump sum subsidy}

In contrast to the fixed subsidy (in which $\mathrm{CFH}$ arranges a fixed payment to Chorus for undertaking each construction stage during this period), under the scheme considered here CFH only makes a lump sum payment to Chorus corresponding to the number of cities that have been completed during a period. That is, the lump sum subsidy scheme requires $\mathrm{CFH}$ to 
learn about how many construction stages in total have been completed by Chorus during each period. Depending on whether the high demand cities could be identified by $\mathrm{CFH},{ }^{87}$ we included two kinds of lump sum subsidy schemes in our model.

\section{The lump sum subsidy scheme not corresponding to the features of cities}

In this section, we would like to discuss the lump sum subsidy scheme which is independent of the demand state of each city. That is, the lump sum payment for each completed city is identical. To avoid confusion, the lump sum subsidy in this section is referred to as the "independent lump sum subsidy", while the lump sum subsidy in the next section is referred to as the "demand dependent lump sum subsidy". We report how the conflict level varies with the level of the independent lump sum subsidy at graph 7.9.

\section{Graph 7.9: The present value of conflict varies with the independent lump sum subsidy}

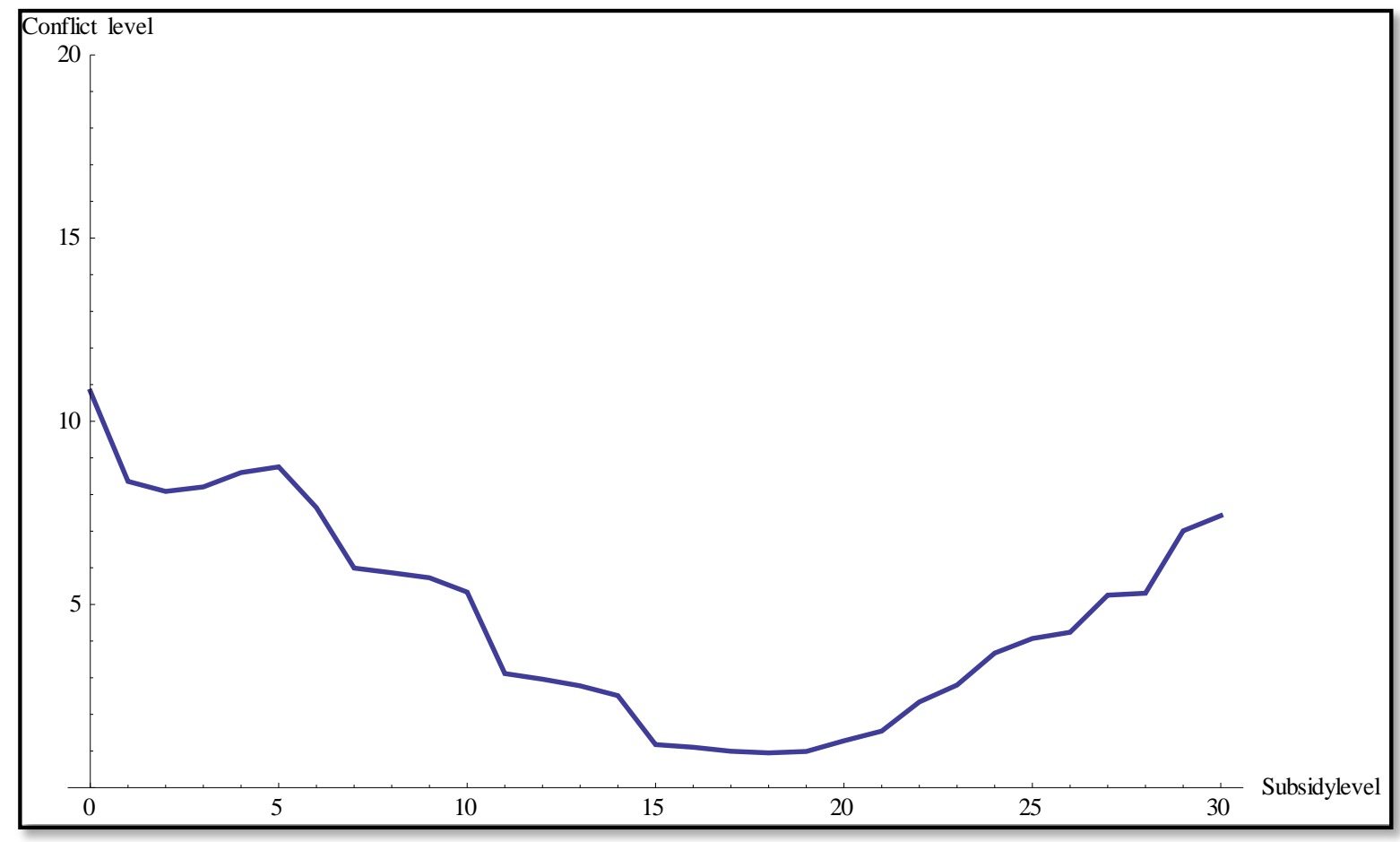

For the independent lump sum subsidy, we find that the corresponding change of conflict level not only shares the same path as that of the fixed subsidy but also has the same minimum level at 0.95 . However, the corresponding optimal level for the lump sum subsidy

\footnotetext{
${ }^{87}$ When CFH could identify the high capital expenditure cities, it could directly navigate from the lump sum subsidy scheme to the proportional subsidy scheme. Therefore, we are interested in the case that demand information about cities could be collected.
} 
equals 18 rather than $8.95{ }^{88}$ Similarly, we find the reason behind the non-monotonic change of conflict level over the independent lump sum subsidy is exactly the same as what we find from the previous two subsidy schemes. Of course, we observe that Chorus's expected numbers of construction stages completed (the timing measure) is increasing with the lump sum subsidy as well.

For the independent lump sum subsidy scheme, we rationalize the minimum level of conflict with the present values to CFH of individual cities (under Chorus' optimal policy). At graph 7.10, we find that PV LL and PV LH follow the same movements as those under the fixed subsidy, and that they are dominated by the PV HH. Similarly, the minimum conflict level should be determined by PV HH, which has a peak when the level of the independent lump sum subsidy equals 18 . Therefore, the minimum conflict level occurs at a subsidy level of 18 .

\section{Graph 7.10: The present values to CFH of the individual cities (under Chorus' optimal policy) vary with the independent lump sum subsidy}

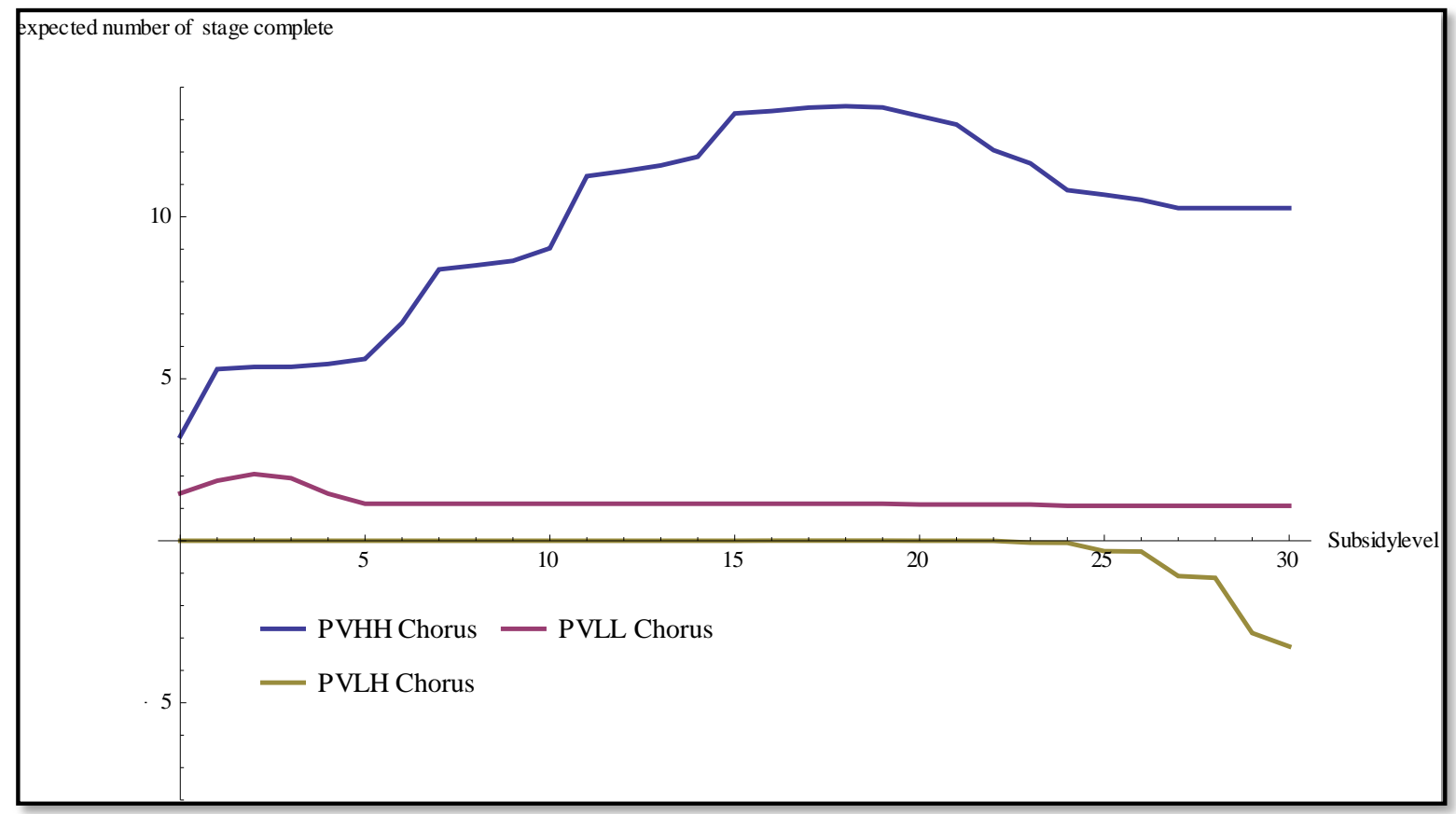

\footnotetext{
${ }^{88}$ This results from the difference of payment timing between fixed and lump sum subsidy schemes. For example, for any specified city, if Chorus would like to start the first construction stage at date 0 and then the second construction stage at date 1 , the fixed subsidy scheme requires CFH making a fixed amount of payment $\mathrm{FS}$ at date 0 and date 1 respectively, while the lump sum subsidy scheme only requires $\mathrm{CFH}$ arranging one lump sum payment LS at date 2 . Therefore, in order to have equivalent present values, the fixed subsidy and lump sum subsidy should meet the condition that $F S+\frac{F S}{R f}=\frac{L S}{R f^{2}}$.
} 
Graph 7.11: The timing conflict measures vary over the independent lump sum subsidy

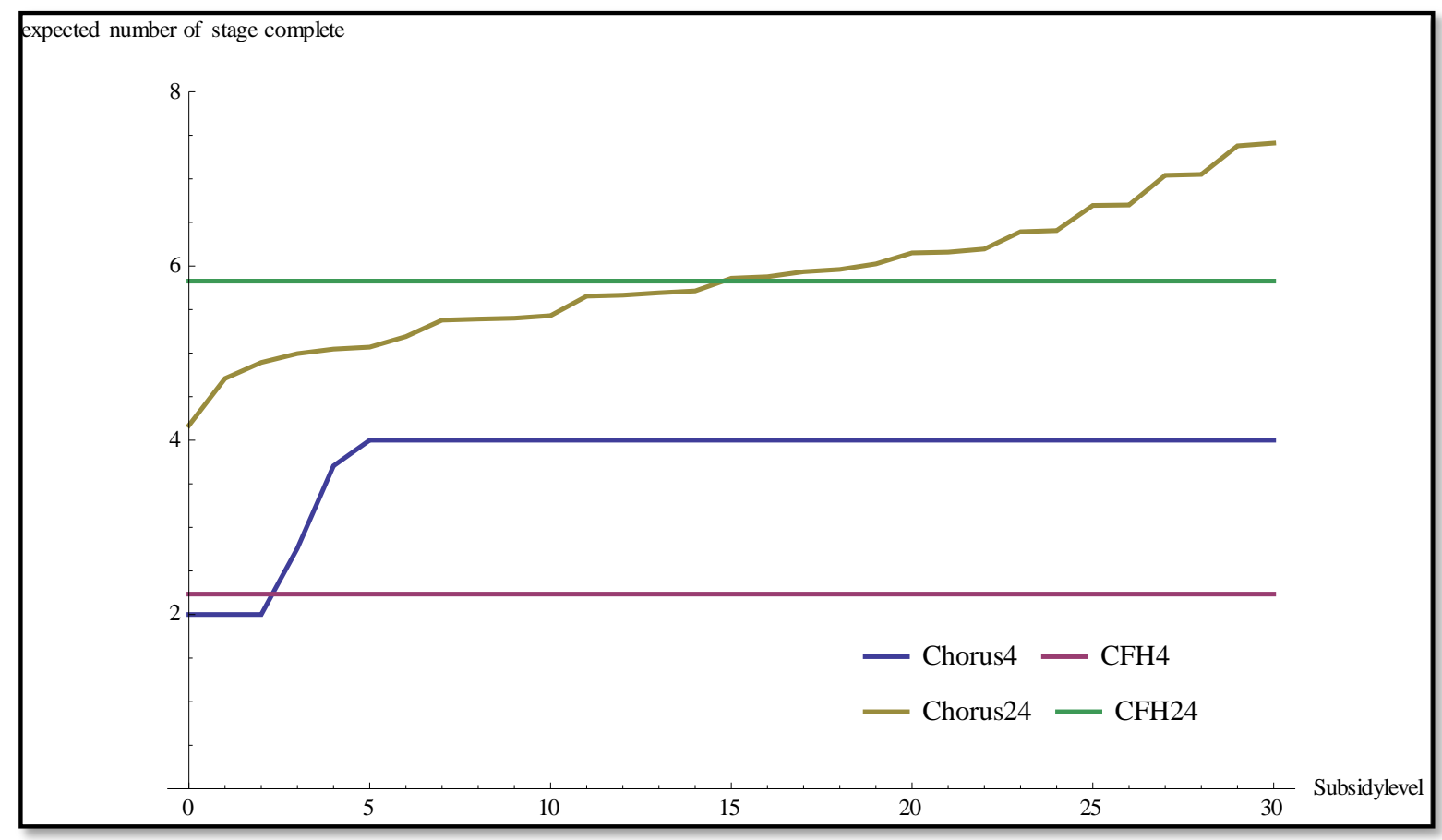

Graph 7.12: The sequencing conflict measures vary over the independent lump sum subsidy

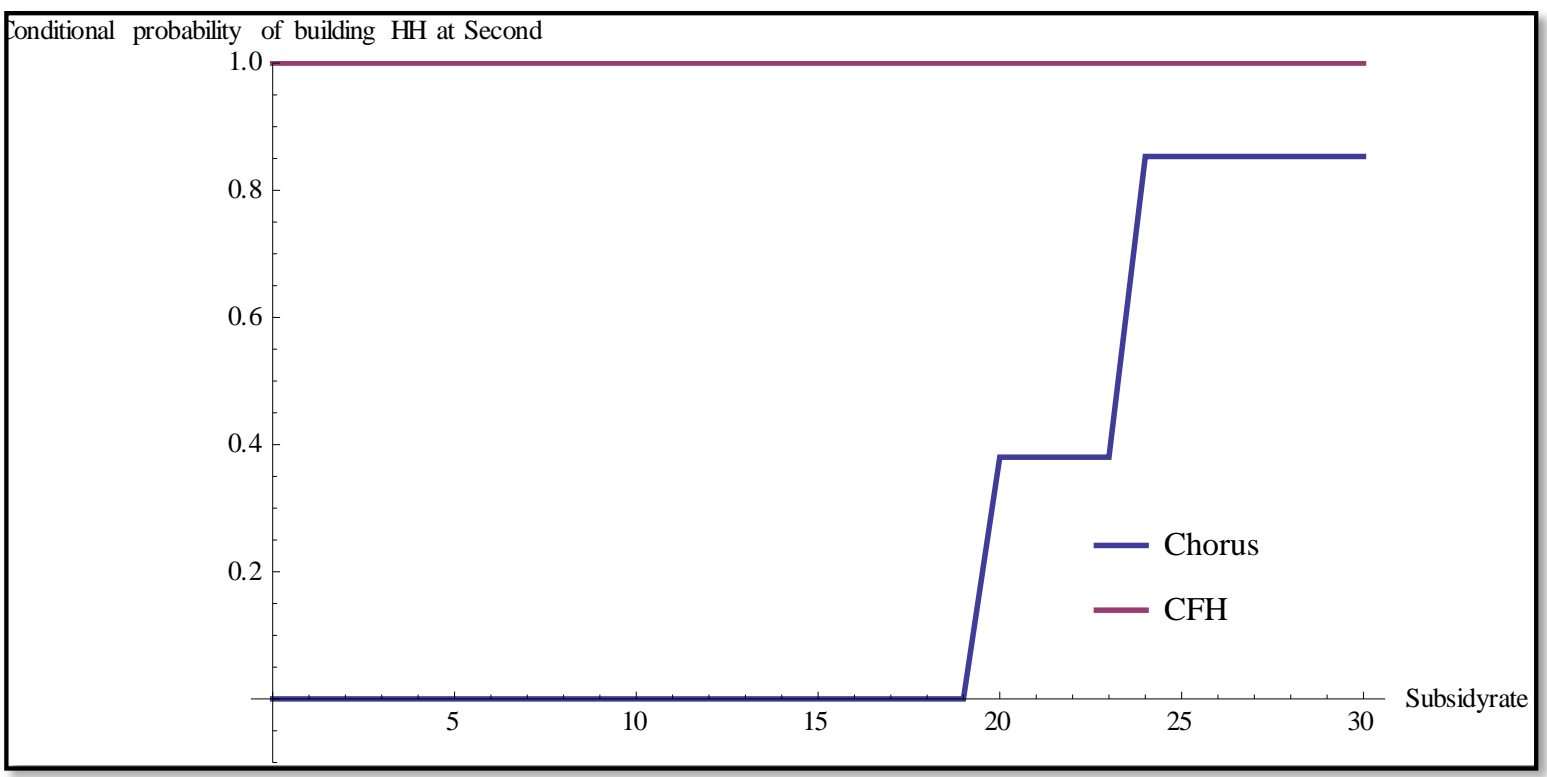

Except for the intersections, we find the timing measures under the independent lump sum subsidy scheme (at graph 7.11) follow the exact same paths as those under the fixed subsidy scheme, which implies that Chorus may have the same construction timing under both the independent lump sum subsidy and fixed subsidy schemes (Clearly, the only difference we 
identify from comparing graph 7.7 and graph 7.11 is that, the corresponding intersections lie on the different subsidy level, which may consistent to the condition $F S+\frac{F S}{R f}=\frac{L S}{R f^{2}}$ ).

At graph 7.12, we find the sequencing conflict under the independent lump sum subsidy scheme follows a similar path to that under the fixed subsidy scheme, meaning that the sequencing conflict decreases over the level of subsidy. The only difference we observe is that the sequencing conflict under the fixed subsidy scheme starts to decrease when the subsidy level equals about 10 , while the sequencing conflict under the lump sum subsidy starts to decrease when the subsidy level equals about 20 .

\section{The lump sum subsidy scheme corresponding to the demand information}

In contrast, the lump sum subsidy scheme in this section generates cash flows that depend on the demand state of each city. That is, the lump sum payment Chorus receives from a high demand city may be different from what it receives from a low demand city. Therefore, we use the label LS1 for the lump sum subsidy to the high demand city, and $L S 2$ for the lump sum subsidy to the low demand city. Accordingly, we have a $3 \mathrm{D}$ rather than $2 \mathrm{D}$ plot for how the conflict level varies with the demand relevant lump sum subsidy scheme at graph 7.13.

\section{Graph 7.13: The conflict level varies with the demand relevant lump sum subsidy}

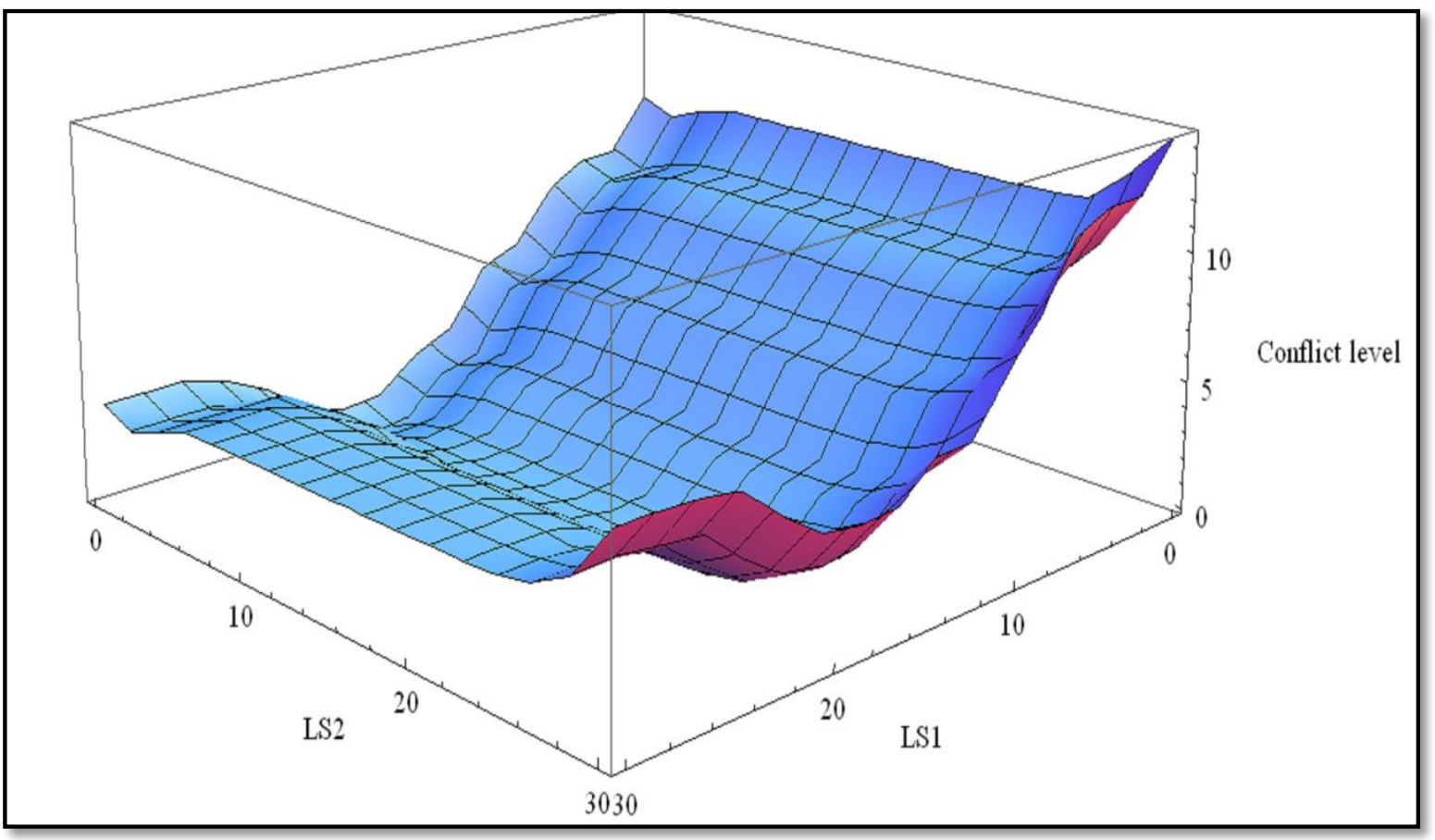


At graph 7.13, we find the conflict level is a non-monotonic function of both $L S 1$ and LS2. However, compared to $L S 2$, the conflict level is more sensitive to the change of $L S 1$, which implies that the lump sum subsidy to high demand cities plays a more important role than the lump sum subsidy to low demand cities in reducing the conflict level. (This may be consistent with the fact that city $\mathrm{HH}$ is the main driver of the conflicts in the baseline case in which there are no incentive scheme.) The minimum conflict level for the demand relevant lump sum subsidy scheme equals 0.07 , which corresponds to $L S 1=18$ and $L S 2=2$. Obviously, the demand relevant lump sum subsidy scheme works better than all the above subsidy schemes (the proportional, fixed and independent lump sum subsidies).

\section{Graph 7.14: The present values to CFH of the individual cities (under Chorus' optimal policy) vary with the demand relevant lump sum subsidy}

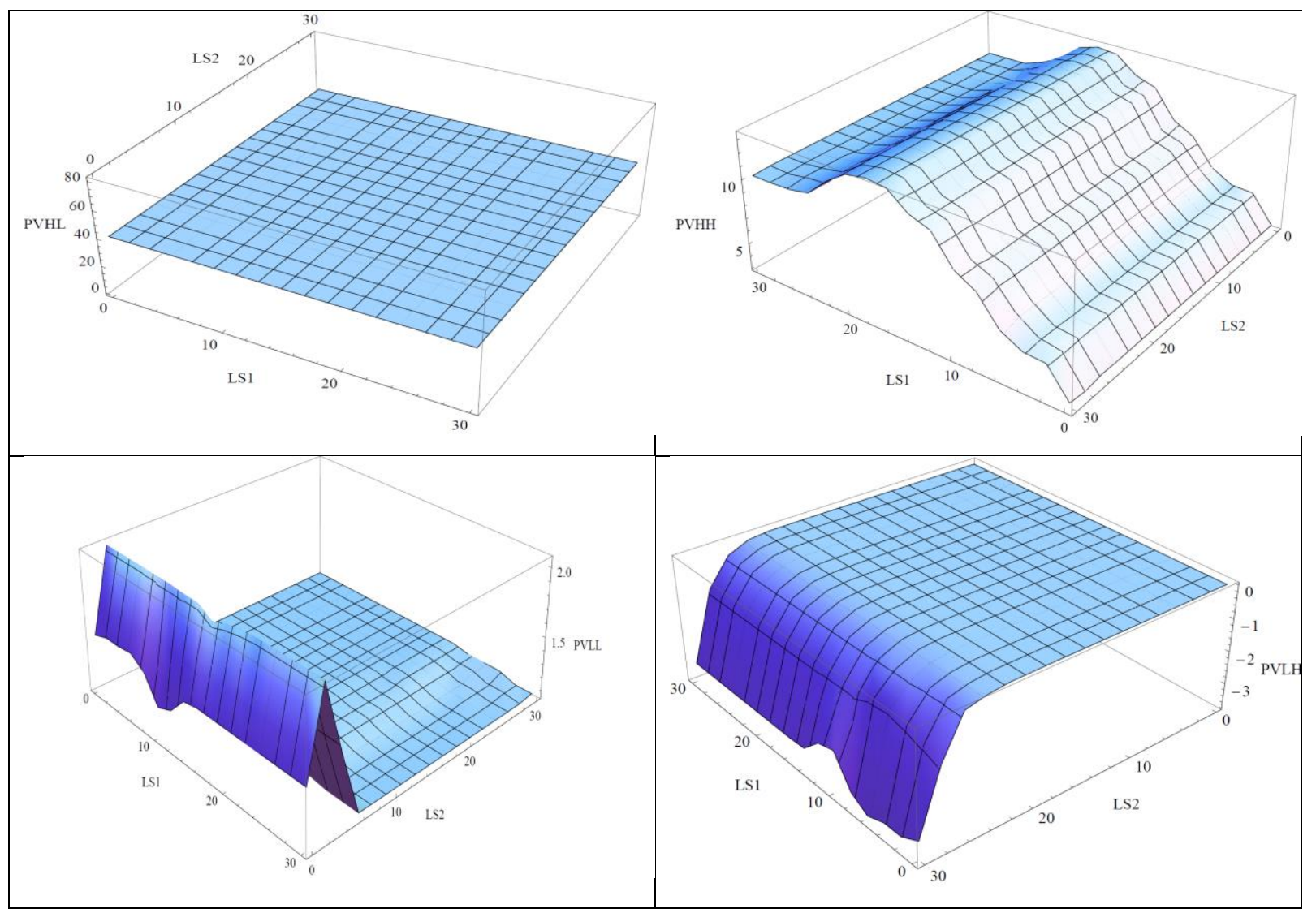

Graph 7.14 plots the present values to $\mathrm{CFH}$ of the individual cities (under Chorus' optimal policy). As we can see from graph 7.14, although city HL has the highest present value, it is hardly affected by the demand relevant subsidy. (The present value to CFH of city HL is a smooth plane at graph 7.14.) The present value of city $\mathrm{HH}$, however, is a non-monotonic function of $L S 1$ but almost independent of $L S 2$. In contrast, the present value of city LL is 
barely affected by $L S 1$ but a non-monotonic function of $L S 2$. This observation does explain why $L S 1$ plays a more important role than $L S 2$ in reducing the conflict level. Recall that the present value of conflict is driven mainly by the present value of city $\mathrm{HH}$. Therefore, the conflict level should be led by the subsidy that determines the present value of city $\mathrm{HH}$, which is $L S 1$. As for the above three subsidy schemes, the present value of city LH is a decreasing function of $L S 2$ when $L S 2$ reaches a certain level. The decreasing present value of city LH is caused by Chorus' overinvestment behavior under the high subsidy. ${ }^{89}$

For the timing conflict measure, we provide the expected numbers of construction stages completed in both 4 and 24 periods at graph 7.15. Instead of having the 3D graph, we plot how the timing conflict measures vary over $L S 1$ for each given level of $L S 2$. (We plot the graphs for $L S 2=0, L S 2=2, L S 2=10$ and $L S 2=30$ ) Similar to the above three subsidy schemes, Chorus becomes more and more aggressive in the network's construction when we increase the demand relevant subsidy. More specifically, for each given level of $L S 2$, the expected numbers of construction stages completed for Chorus (both in 4 and 24 periods) are increasing with $L S 1$. For each given level of $L S 1$, the expected numbers of construction stages completed for Chorus (both in 4 and 24 periods) are increasing with $L S 2$. When we set $L S 2$ equal to 0 or 2 (the first two panels in graph 7.15), the expected numbers for Chorus in 4 periods reflect how the construction timing of city $\mathrm{HH}$ increases when $L S 1$ is relatively big, while the expected numbers for Chorus in 24 periods reflect how the construction timing of city $\mathrm{HH}$ increases when $L S 1$ is relatively small. ${ }^{90}$ Nevertheless, if we shift $L S 2$ to 10 or 30 (The last two panels in graph 7.15), only the expected numbers for Chorus in 24 periods reflect how the construction timing of city $\mathrm{HH}$ increases with $L S 1$. On the other hand, keeping $L S 1$ fixed, we find that the increase of $L S 2$ is going to level up the expected numbers for Chorus in 24 periods without changing its trend.

\footnotetext{
${ }^{89}$ This shares the same argument as that in the footnote 86.

${ }^{90}$ City HL is barely affected by any subsidy. Therefore, the lump sum subsidy for high demand cities mainly affects city HH's construction timing. When the subsidy to high demand cities is relatively low, the construction of city $\mathrm{HH}$ is marginal to Chorus. In contrast, when the subsidy to high demand cities is relatively high, the construction of city $\mathrm{HH}$ becomes very profitable to Chorus. Since the expected numbers in 4 periods are sensitive to the profitable city, while the expected numbers in 24 periods are sensitive to the marginal city. The expected numbers in 4 periods should correspond to the city $\mathrm{HH}$ under the high subsidy, while the expected numbers in 24 periods should correspond to the city $\mathrm{HH}$ under the low subsidy.
} 
Graph 7.15: The timing conflict measures vary over the demand relevant lump sum subsidy

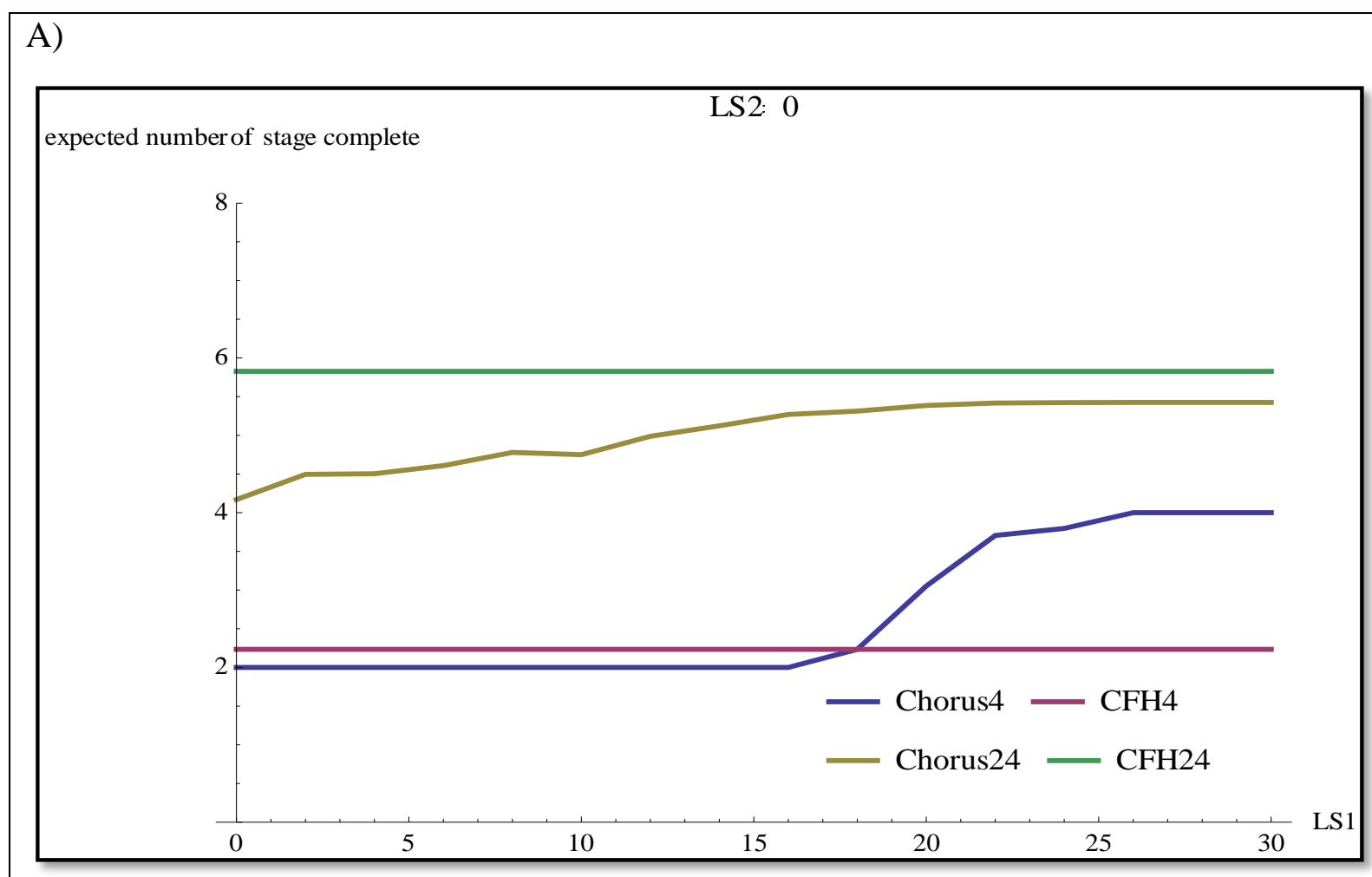

B)

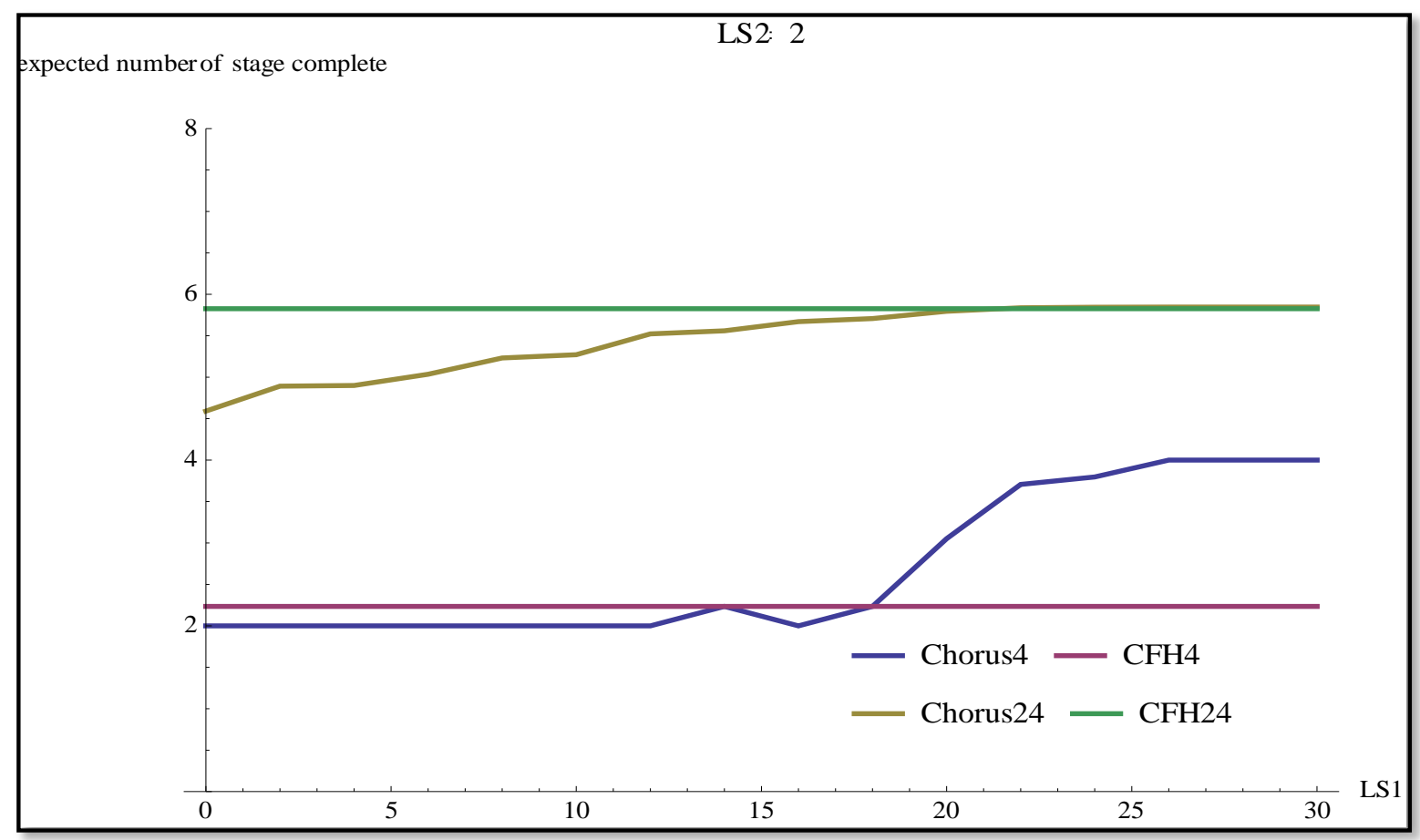




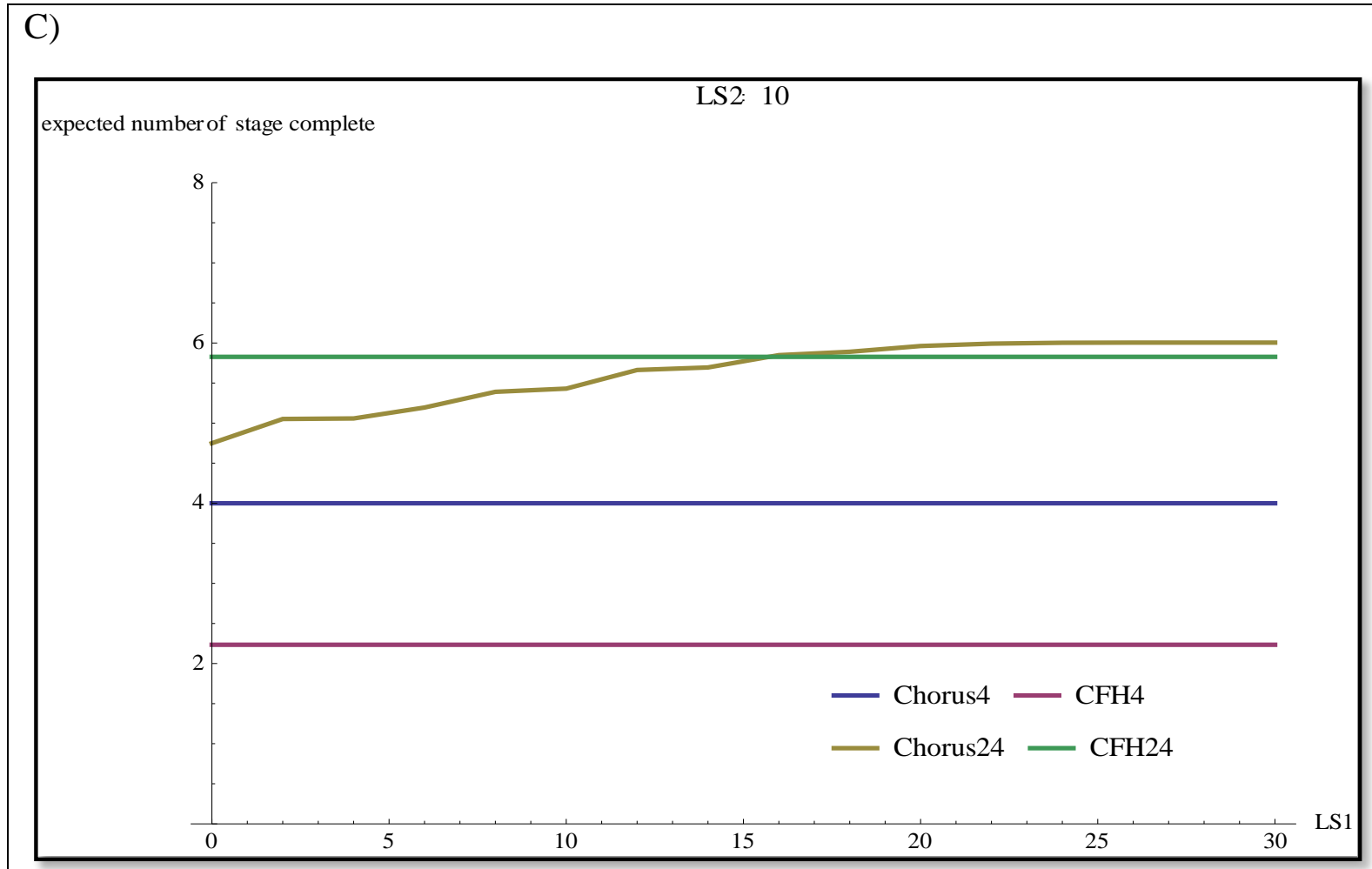

D)

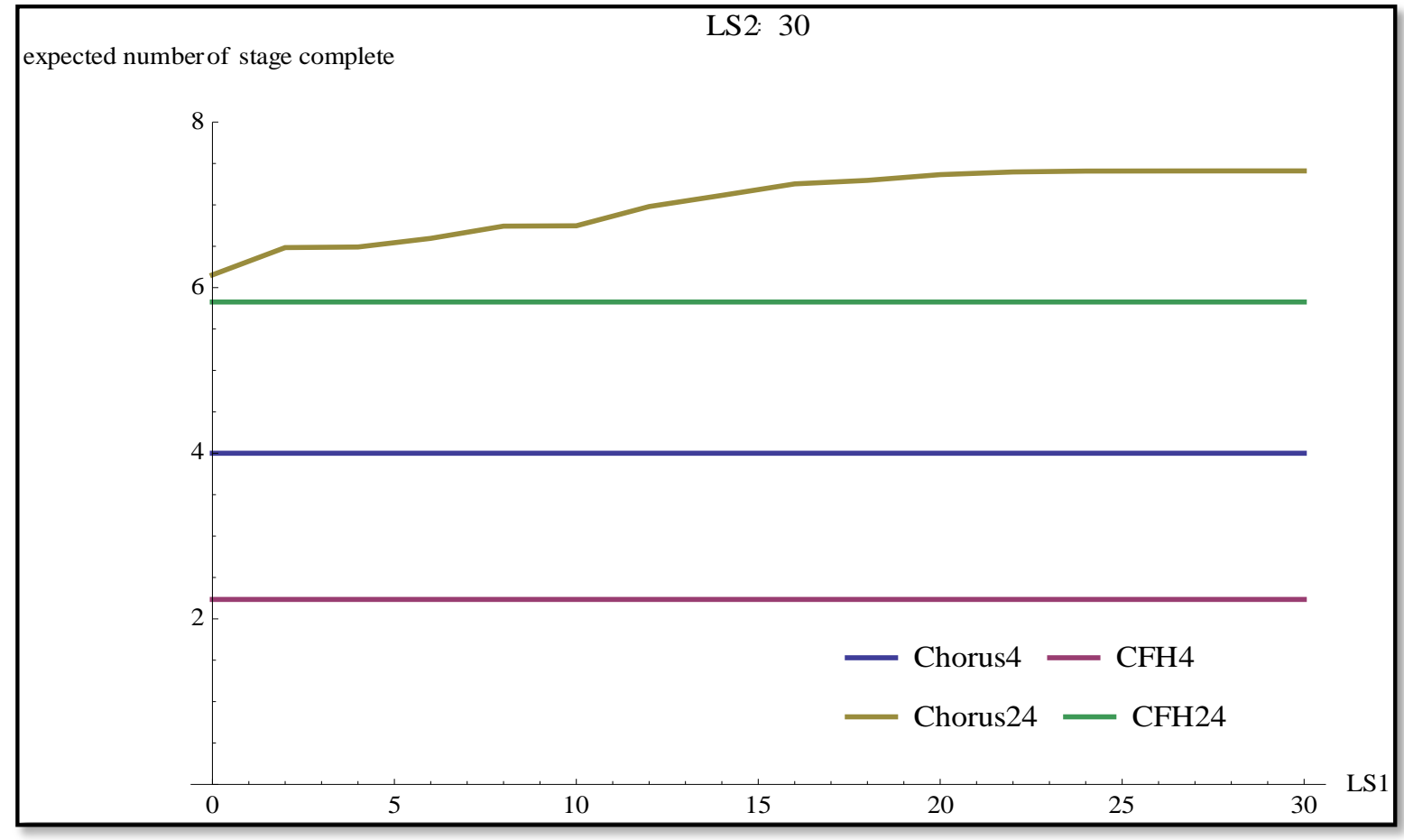




\section{Graph 7.16: The sequencing conflict measures vary over the demand relevant lump sum subsidy}

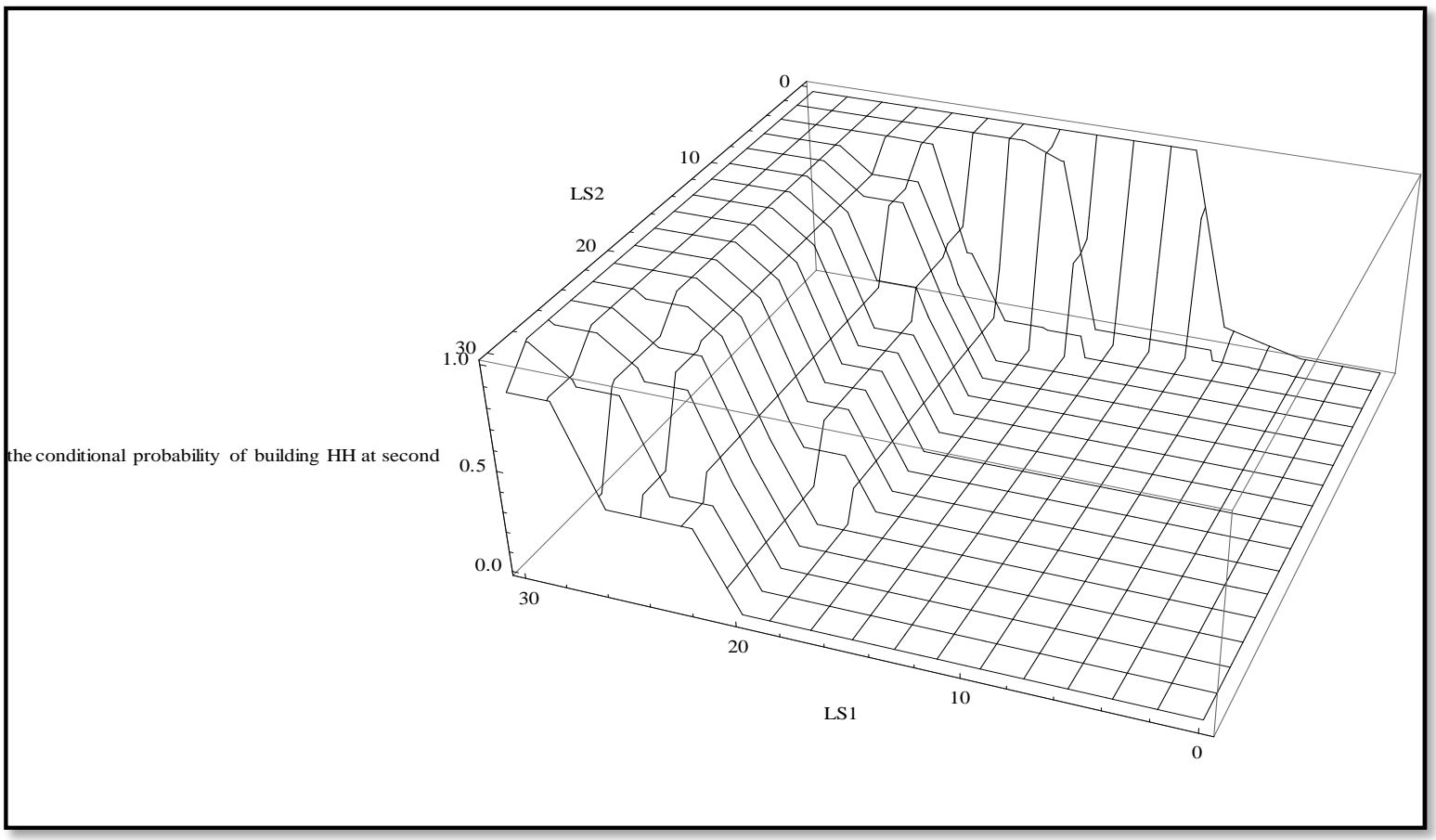

Graph 7.16 plots the conditional probability that Chorus wants to complete city $\mathrm{HH}$ second providing that at least two cities will be finished. ${ }^{91}$ When there is not any subsidy (both $L S 1$ and $L S 2$ equal 0), the conditional probability for Chorus equals 0. If we keep LS2 constant, the corresponding conditional probability for Chorus monotonically increases over LS1. If we keep $L S 1$ constant instead (when $L S 1$ is bigger than 8), the corresponding conditional probability for Chorus, however, monotonically decreases over $L S 1$. The logic behind this is clear. That is, for any given level of $L S 2$, the increase of $L S 1$ encourages Chorus to construct city HH more aggressively (the construction timing of city HH increases), which indicates the city $\mathrm{HH}$ has a higher chance of being finished earlier than the city LL. In contrast, for any given level of $L S 1$ (when $L S 1$ is bigger than 8), the increase of $L S 2$ encourages Chorus to construct city LL more aggressively (the construction timing of city LL increases), which indicates that city LL has more chance of being finished earlier than city $\mathrm{HH} .{ }^{92}$ Besides, we may realize that, the larger $L S 2$ is, the more $L S 1$ is required to make the conditional

\footnotetext{
${ }^{91}$ Since the subsidy does not affect CFH's construction behaviours, the conditional probability that CFH wants to complete city $\mathrm{HH}$ second providing that at least two cities will be finished always equals 1 , which is a smooth plane. Therefore, we only plot the conditional probability for Chorus.

${ }^{92}$ When LS1 is smaller than 8, the corresponding conditional probability for Chorus is close to zero, which cannot be reduced further when $L S 2$ increases. (Since the increase of $L S 2$ speeds up the construction timing of city $L L$, it only reduces the conditional probability when it is big)
} 
probability to be 1 . This is because, with a larger $L S 2$, Chorus tends to construct city LL faster, which makes it harder to be overtaken by city $\mathrm{HH}$ in construction timing. Therefore, the corresponding $L S 1$ (the subsidy component that makes the city HH overtake city LL in construction timing) is required to be larger. In summary, the sequencing conflict is more likely to disappear when the lump sum subsidy for the high demand cities (LS1) is high but the lump sum subsidy for the low demand cities (LS2) is low.

\subsection{The sensitivity analysis of the incentive schemes}

For each incentive scheme, the sensitivity analysis of the profile of the optimal subsidy may help us have a better understanding about how the level of optimal subsidy (correspondingly the level of minimum conflict) is affected by varying several parameters (Con, DR, $\boldsymbol{C R}$ and $\sigma$ ). Therefore, we could have some comments about the stability of the optimal subsidy under the different subsidy schemes. However, we only provide the sensitivity analysis for the proportional subsidy and independent lump sum subsidy schemes. ${ }^{93}$

\section{1) The sensitivity analysis of the proportional subsidy scheme}

Graph 7.17 plots the results of the sensitivity analysis on several important parameters (Such as $\boldsymbol{C o n}, \boldsymbol{D R}, \boldsymbol{C R}$ and $\boldsymbol{\sigma}$ ) under the proportional subsidy scheme. That is, the conflict level at graph 7.17 is not only a function of the proportional subsidy rate but also a function of one of these parameters. For the particular level of each parameter, the optimal subsidy rate is the subsidy rate corresponding to the minimum conflict level on that curve. And then, as we vary the parameter, we can track out how sensitive the optimal subsidy rate is to this parameter. At the top left corner of graph 7.17, we find the conflict level is insensitive to the subsidy rate when $\mathbf{C o n}$ is small, which means the optimized conflict level is indifferent from those non-optimized conflict levels. However, when Con is getting bigger, the conflict level under each given subsidy rate is becoming higher, more importantly, the less the given subsidy rate is the more the corresponding conflict level increases, which indicates that the conflict level becomes more sensitive to the subsidy rate. The logic behind the increase of Con is quite straightforward. When we raise Con, the corresponding consumer surplus is

\footnotetext{
${ }^{93}$ In order to conduct the sensitivity analysis for the demand relevant lump sum subsidy scheme, we need to plot a 4D graph, which is too complicated for our current research. Besides, the results of sensitivity analysis from the demand relevant lump sum subsidy scheme are quantitatively similar to that in other subsidy schemes. For the fixed subsidy scheme, it looks almost identical to the independent lump sum subsidy scheme except for the higher monitoring costs. Therefore, we skip the above two subsidy schemes in our sensitivity analysis.
} 
increasing according to equation 1. Consequently, the conflict level between $\mathrm{CFH}$ and Chorus is getting bigger since the consumer surplus is the main source of conflicts. At the bottom left corner of graph 7.17 , we provide the result of the sensitivity analysis for the cost ratio $(\boldsymbol{C R})$. That is, when $\boldsymbol{C R}$ is quite high (Such as $\boldsymbol{C R}=6$ ), the conflict level is quite low and optimized at a subsidy rate of about 0.2 . As the cost ratio falls to 4 or 5 , there is little change in the conflict level and the optimal subsidy rate still lies on about 0.2. However, once the cost ratio reaches about 2 or 3 , we observe two changes. First, the optimal subsidy rate is increasing (which is about 0.4 subsidy rate). Second, the level of conflict is increasing. Namely, even with a subsidy rate of 0.2 (which is the optimal subsidy rate when $\boldsymbol{C} \boldsymbol{R}$ is bigger), the conflict is relatively substantial when the cost ratio becomes quite low. When that happens, the optimal subsidy rate needs to be higher. The reason behind this is clear. When the cost ratio is relatively high, ${ }^{94}$ both $\mathrm{CFH}$ and Chorus found the construction of city $\mathrm{HH}$ is so unprofitable that the disagreements ${ }^{95}$ between these two cities on constructing city HH disappear.

At graph 7.17, we post the result of sensitivity analysis on the demand ratio $(\boldsymbol{D R})$ at the top right corner. When $\boldsymbol{D} \boldsymbol{R}$ is extremely high (When $\boldsymbol{D} \boldsymbol{R}$ ranges between 8 and 11), the conflict level is quite low and the corresponding optimal subsidy rate lies on about 0.25 . However, once the demand ratio reduces to about 3 or 4 , we find both the conflict level and optimal subsidy rate become larger. ${ }^{96}$ The reason behind the lumpiness of the graph is quite similar to that of the cost ratio. When the demand ratio is very high (When $\boldsymbol{D R}$ ranges between 8 and 11), both parties consider the construction of city $\mathrm{HH}$ is so profitable that both of them would like to build it very quickly. In contrast, when the demand ratio becomes very small (that is, close to $\boldsymbol{D R}=1$ ), both parties consider the construction of city $\mathrm{HH}$ is so unprofitable that neither of them would like to build the city HH up. Therefore, the disagreements between $\mathrm{CFH}$ and Chorus on constructing city $\mathrm{HH}$ vanish either when the demand ratio is very large or very small, leaving the lumpiness between these two extremes.

At the bottom right corner of graph 7.17, we show the result of sensitivity analysis of the level of volatility $(\sigma)$. That is, if we increase the level of volatility, the conflict level under a high subsidy rate increases, while the conflict level under a low subsidy rate decreases. ${ }^{97}$ As

\footnotetext{
${ }^{94}$ What we mean is that the cost ratio is higher than the demand ratio.

${ }^{95}$ The disagreement is for both the sequencing and timing conflicts.

${ }^{96}$ The optimal subsidy rate shifts from about 0.25 to about 0.45 .

${ }^{97}$ As we can see, the conflict level under the 0.8 subsidy rate decreases over the volatility, while the conflict level under the 0.2 subsidy rate increases over the volatility.
} 
a result, the optimal subsidy rate increases from 0.4 to 0.65 when we increase $\sigma$ from 0.1 to 0.2. In contrast, the corresponding minimum conflict level reduces slightly. In summary, under the proportional subsidy scheme, the conflict level becomes more sensitive to the subsidy rate in two cases. A) When either $\boldsymbol{D} \boldsymbol{R}$ or $\boldsymbol{C} \boldsymbol{R}$ turns out to be modest; B) When either Con or $\sigma$ turns out to be large. However, in practice, the CFH may hope the conflict level is insensitive to the subsidy rate (In other words, $\mathrm{CFH}$ may hope that they are not in case A or case B). This is because the more insensitive the subsidy is the harder it is for CFH to have a bad incentive plan to manage the conflict. ${ }^{98}$

\section{Graph 7.17: The sensitivity analysis of several interesting parameters under the proportional subsidy scheme.}

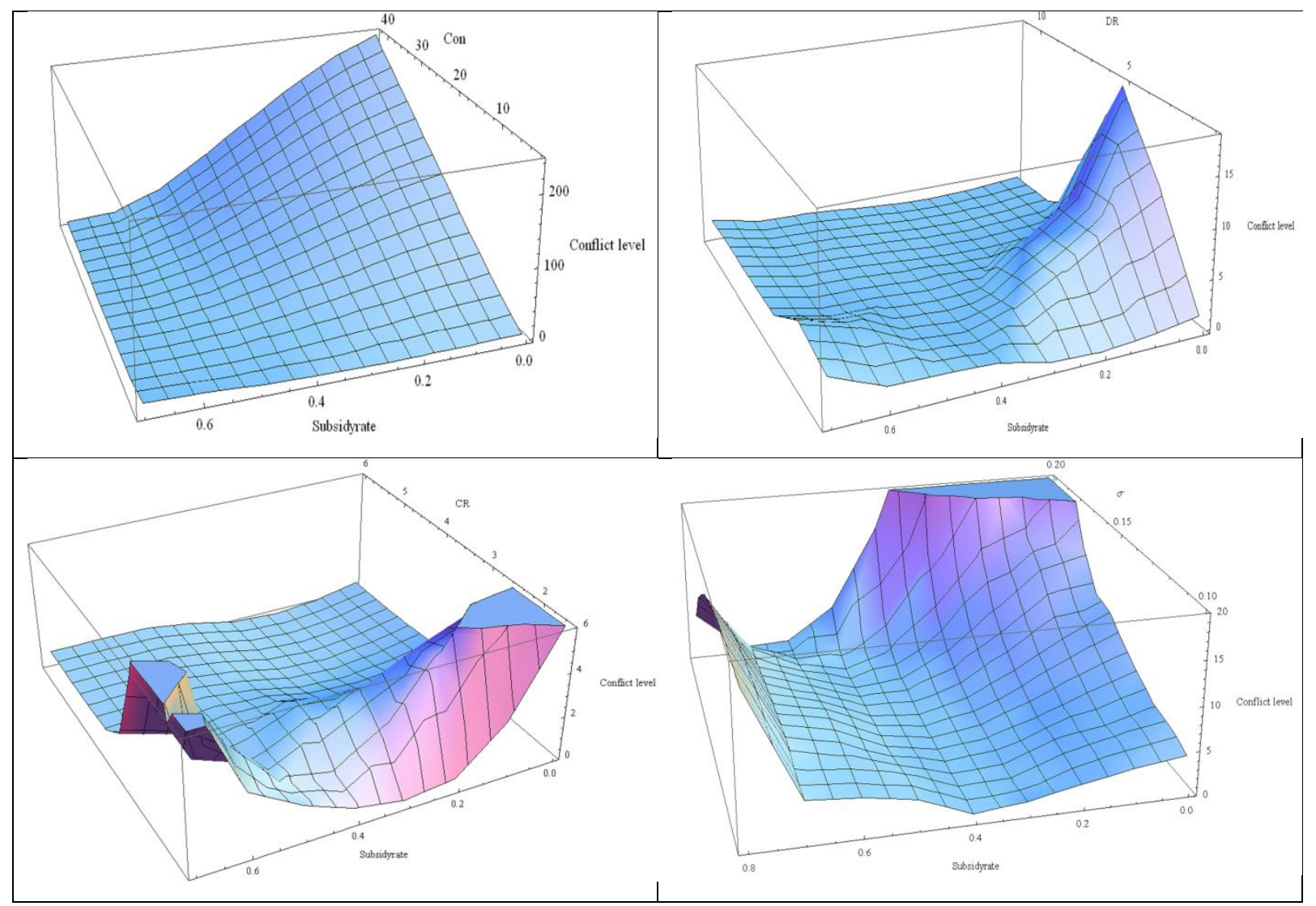

\footnotetext{
${ }^{98}$ For example, when the level of volatility equals 0.2 , the conflict level is quite sensitive to the subsidy rate. Therefore, $\mathrm{CFH}$ is going to be concerned by the very high level of conflict that occurs they get the subsidy rate slightly wrong. In contrast, when the level of volatility equals 0.1 , they are less worried about that since the level of conflict cannot go too high even when the subsidy rate differs significantly from its optimal value.
} 


\section{2) The sensitivity analysis of the independent lump sum subsidy scheme}

Graph 7.18 plots the results of the sensitivity analysis on several important parameters (Con, $\boldsymbol{D} \boldsymbol{R}, \boldsymbol{C R}$ and $\boldsymbol{\sigma})$ under the independent lump sum subsidy scheme. Similarly, we report the results of sensitivity analysis to $\boldsymbol{C o n}, \boldsymbol{D R}, \boldsymbol{C R}$ and $\boldsymbol{\sigma}$ at the top left corner of graph 7.18 , the top right corner of graph 7.18, the bottom left corner of graph 7.18 and the bottom right corner of graph 7.18 respectively. The results of the sensitivity analysis to $\boldsymbol{C o n}, \boldsymbol{D R}, \boldsymbol{C R}$ and $\sigma$ under the independent lump sum subsidy scheme are quite similar to those under the proportional subsidy scheme. ${ }^{99}$ The only difference we observe is that the corresponding optimal subsidy values are different between these two subsidy schemes.

\section{Graph 7.18: The sensitivity analysis of several interesting parameters under the} independent lump sum subsidy scheme.

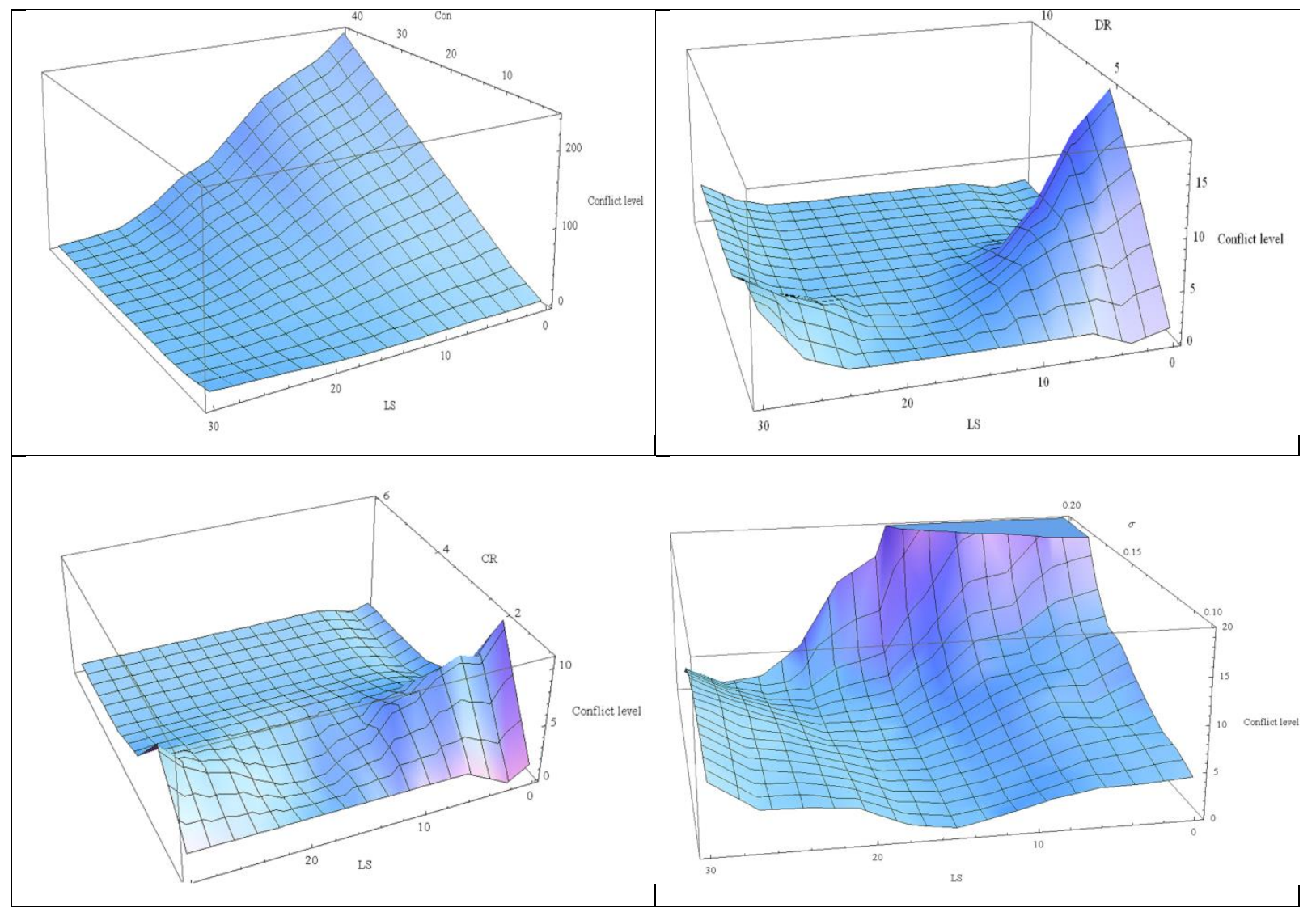

\footnotetext{
${ }^{99}$ The readers may find the results from the proportional subsidy scheme are smoother than those from the independent lump sum subsidy scheme. We believe this occurs because we plot more values of the independent lump sum subsidy than the proportional subsidy in our sensitivity analysis.
} 


\subsection{The comparison of subsidy schemes}

In this section, we compare the above four subsidy schemes' ability to reduce the possible conflicts. More importantly, we are going to discuss their differences in monitoring costs and information requirements.

Clearly, the demand relevant lump sum subsidy scheme has the best performance in achieving the minimum conflict level among all subsidy schemes. The proportional subsidy scheme, however, works less efficiently than the demand relevant lump sum subsidy scheme but more efficiently than both the fixed subsidy scheme and independent lump sum subsidy scheme in reducing the possible conflicts. Apart from that, the fixed subsidy and independent lump sum subsidy schemes have been found to be equally efficient in reducing the conflict level. Actually, we found that Chorus is indifferent between the fixed subsidy and independent lump sum subsidy schemes when the condition $F S+\frac{F S}{R f}=\frac{L S}{R f^{2}}$ has been met, ${ }^{100}$ which means the options to suspend or accelerate the construction process are worthless. $^{101}$

Now, we are going to explain what causes the above subsidy schemes to have different performances in reducing the conflict level. A comparison from graph 7.2, graph 7.6 and graph 7.10 explains why the proportional subsidy scheme works better than both the fixed subsidy and independent lump sum subsidy in achieving the minimum level of conflict. That

${ }^{100}$ In order to test this argument, we set FS (the fixed subsidy payment) and $L S$ (the lump sum subsidy payment) with condition $F S+\frac{F S}{R f}=\frac{L S}{R f^{2}}$ to make them comparable. Therefore, we can estimate not only the present value of conflict under the fixed subsidy but also the present value of conflict under the corresponding lump sum subsidy. (For any given lump sum subsidy, we can calculate the corresponding fixed subsidy directly through the condition $F S+\frac{F S}{R f}=\frac{L S}{R f^{2}}$ and then the corresponding conflict level.) The difference of conflict level under the above two subsidy schemes could be applied to test our argument. That is, the difference should equal zero when Chorus has identical behaviors under these two subsidy schemes and nonzero otherwise. We found the difference of conflict level always equals zero when we vary the $L S$. Besides, we extend our test by varying both $L S$ and one of the interesting parameters (Con, volatility, DR, CR) to see whether our argument only holds for the baseline case. Similarly, we found the difference of conflict level equals zero all the time.

${ }^{101}$ As we mention in chapter 5 , a shift from the one period model to the two-period model allows the existence of extra flexibilities. That is, the decision maker in our model is allowed to suspend or accelerate the construction process. However, these extra flexibilities are valueless if Chorus reacts indifferently under fixed and independent lump sum subsidy schemes, which is our case. This is because, if Chorus never actually exercises these extra flexibilities, the fixed subsidy it receives must share the same present value as that of the independent lump sum subsidy. (Since we set $F S$ and $L S$ with condition $F S+\frac{F S}{R f}=\frac{L S}{R f^{2}}$, the present value of the fixed subsidy does not equal the present value of the independent lump sum subsidy when the private party is likely to exercise the extra flexibilities) Therefore Chorus will take the exact same actions in the construction under these two subsidy schemes. (As a result, the conflict levels are equal under these two subsidy schemes) 
is, all subsidy schemes can maximize the PV HH at about the same amount ${ }^{102}$, however, the corresponding PV LL under the fixed and independent lump sum subsidies are lower than those under the proportional subsidy. In dollar terms, the proportional subsidy means we can have a relatively high subsidy for city $\mathrm{HH}$ and a relatively low subsidy for city LL. In contrast, Chorus receives the same values of subsidy for each city (either it is under the fixed subsidy scheme or the independent lump sum subsidy scheme), which implies it either under-subsidizes city HH or over-subsidizes city LL. Therefore the proportional subsidy scheme has the better match than the other two subsidy schemes in subsidizing the different cities. The reason that the demand relevant lump sum subsidy works even better than the proportional subsidy in reducing the conflict is due to its extra degree of freedom (The demand relevant lump sum subsidy enjoys two degree of freedom in subsidizing the cities, while the other three subsidy schemes only have one degree of freedom in subsidizing the cities). Since the conflict is mainly driven by city HH (a high demand city) and city LL (a low demand city), ${ }^{103}$ the demand relevant lump sum is able to fit both cities very well with different levels of subsidy, which solves the mismatching problem in the proportional, fixed and independent lump sum subsidy schemes. More specifically, in the other three subsidy schemes, although the conflicts of city HH and city LL could be reduced almost completely with the corresponding "optimal" levels of subsidy, the corresponding "optimal" level of subsidy for a high demand city is never a good match to the corresponding "optimal" level of subsidy for a low demand city. ${ }^{104}$ However, with the extra degree of freedom, there must be one combination of $L S 1$ and $L S 2$ that can reduce both the conflicts of city $\mathrm{HH}$ and city LL completely. Therefore, the demand relevant lump sum subsidy has the best performance among all subsidy schemes.

Of course, there are different levels of information required to implement these four subsidy schemes. For example, for the proportional subsidy, CFH is required to learn Chorus' "actual" capital expenditure during each construction period in order to set the "optimal" monetary transfer. On the other hand, each city's demand state is required to be identified by $\mathrm{CFH}$ under the demand relevant lump sum subsidy scheme. Although both the fixed subsidy

\footnotetext{
${ }^{102}$ Since there is one degree of freedom for these three subsidy schemes, they should have the same performance in optimizing one value (Here is the PV HH).

${ }^{103}$ For our baseline parameters, city HL is so profitable that both parties would like to build it up very quickly, while city LH is such a bad investment that neither party would like to construct it unless the demand turns out to be very good. Accordingly, CFH and Chorus will have almost no conflict in city HL and city LH. Therefore, the present value of conflict is mainly affected by two parties' different behaviour in constructing city $\mathrm{HH}$ and city LL.

${ }^{104}$ A comparison from graph7.2, graph 7.6 and graph 7.10 well supports this argument.
} 
and independent lump subsidy schemes require no specific information (which is about demand or capital expenditure) to set up the monetary transfer, in each period, the fixed subsidy generally requires more information about the completion of UFB construction than the independent lump sum subsidy. More specifically, the independent lump subsidy scheme requires learning how many final construction stages in total have been completed in each period, while the fixed subsidy scheme not only needs to learn about the final construction stages but also how many of the first construction stages have been finished in each period. However, when relating the information requirement to the efficiency in reducing the conflict level, we found the specific information helps reduce the conflict level while the extra information about the completion of UFB construction does not. ${ }^{105}$ Certainly, the extra information requirements may not be a "free lunch" to CFH due to the extra monitoring costs. ${ }^{106}$ Therefore, any shift among these subsidy schemes is justified if the margin of conflict it reduces dominates the margin of extra monitoring cost. In other words, when shifting subsidy schemes the maximum cost for extra monitoring should be less than the present value of the reduced conflict. Therefore, for our baseline parameters, the extra monitoring cost cannot exceed 0.88 if $\mathrm{CFH}$ is to benefit for shifting from the independent lump sum subsidy scheme to the demand relevant lump sum subsidy scheme, while the maximum extra monitoring cost cannot exceed 0.21 if $\mathrm{CFH}$ is to benefit for shifting from the independent lump sum subsidy scheme to the proportional subsidy scheme. Further on, when CFH needs to choose from the demand relevant lump sum subsidy and proportional subsidy schemes, we believe the demand relevant lump sum subsidy scheme dominates the proportional subsidy scheme. This is because, compared to the proportional subsidy scheme, the demand relevant lump sum subsidy scheme not only has better performance in reducing the conflict level but also suffers less from the extra monitoring costs. ${ }^{107}$ In the extreme case that a change of subsidy scheme does not reduce any conflict, any extra monitoring cost will

\footnotetext{
${ }^{105}$ Certainly, the heterogeneity of each city in demand and cost state is the main source of conflict. Therefore, any information which can help CFH better identifying these states should improve the power of incentive scheme.

${ }^{106}$ A shift from the independent lump subsidy scheme to the fixed subsidy scheme raises an extra cost to CFH in verifying how many of first stage constructions in total are under construction (counting the numbers of first stage construction). However, a shift from the fixed subsidy scheme to the proportional subsidy scheme causes an extra cost to CFH in verifying the "actual" construction expenditure for each undertaking construction stage. Further on, a shift from the independent lump subsidy scheme to the demand relevant lump sum subsidy scheme causes an extra cost to CFH in verifying whether the cities finished in the last period are high demand or low demand.

${ }^{107}$ Generally speaking, it is easier for the third party to collect the demand information than the information about capital expenditure. For example, in a bridge construction, the public party can figure out the traffic flow easily by setting up a group of security cameras. However, it is more difficult for them to identify whether the contractor inflated the construction expenditure.
} 
be too much. Therefore a shift from the independent lump sum subsidy scheme to the fixed subsidy scheme should never be preferred.

\subsection{The study of fine schemes}

Similarly to the subsidy schemes, CFH could discipline Chorus in the UFB project through a fine scheme. That is, Chorus could be required to pay a penalty whenever it fails a target designated by $\mathrm{CFH}$. We start our study of fine schemes by considering a simple fine scheme.

\subsubsection{The simple fine scheme}

Under the simple fine scheme, $\mathrm{CFH}$ could penalize Chorus when Chorus fails the target at date 24 (which is the terminal date). The amount of fine Chorus needs to pay depends on the number of cities not yet finished. For example, if CFH wants Chorus to finish all four cities, then Chorus will be required to pay $\mathrm{CFH}$ an amount Finelevel for each city not completed on or before date 24. Apart from the timing of the monetary transfer, the simple fine scheme may look like the independent lump sum subsidy scheme with the negative subsidy payment. We report how the conflict level varies with the simple fine scheme at graph 7.19.

\section{Graph 7.19: The present value of conflict varies with the simple fine (target date at} date 24)

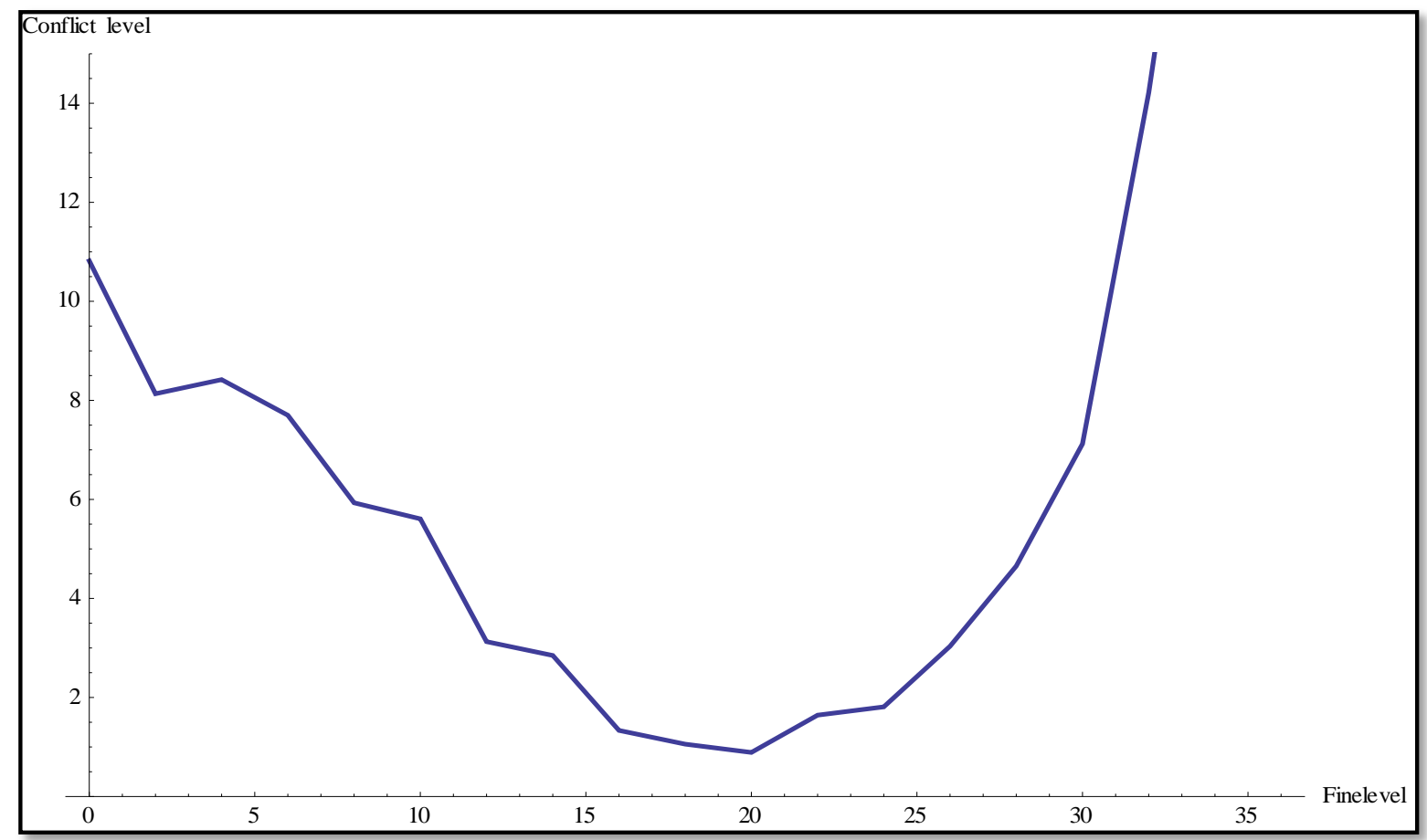


As we may find from graph 7.19, the conflict level under the simple fine scheme shares the same non-monotonic relationship to the level of fine as the subsidy schemes. However, the conflict level under the simple fine scheme is minimized at 0.891 when the level of the fine equals 20. The logic behind the non-monotonic change of the conflict level is straightforward. When the level of fine goes up, Chorus acts more and more aggressively in the UFB network's construction in order to avoid the penalty. Recall that the increase of Chorus's aggression in construction first results in a better match between the two parties' construction preferences. ${ }^{108}$ Therefore, the conflict level decreases in the first half of graph 7.19. However, after the level of fine reaches a certain level, the further increase of the fine forces Chorus to act more aggressively in the UFB network's construction than CFH would like. ${ }^{109}$ Consequentially, the increased deviation between the construction preferences of the two parties fuels up the level of conflict in the second half of graph 7.19.

\section{Graph 7.20: The present values to CFH of the individual cities (under Chorus' optimal policy) vary with the simple fine (target date at date 24)}

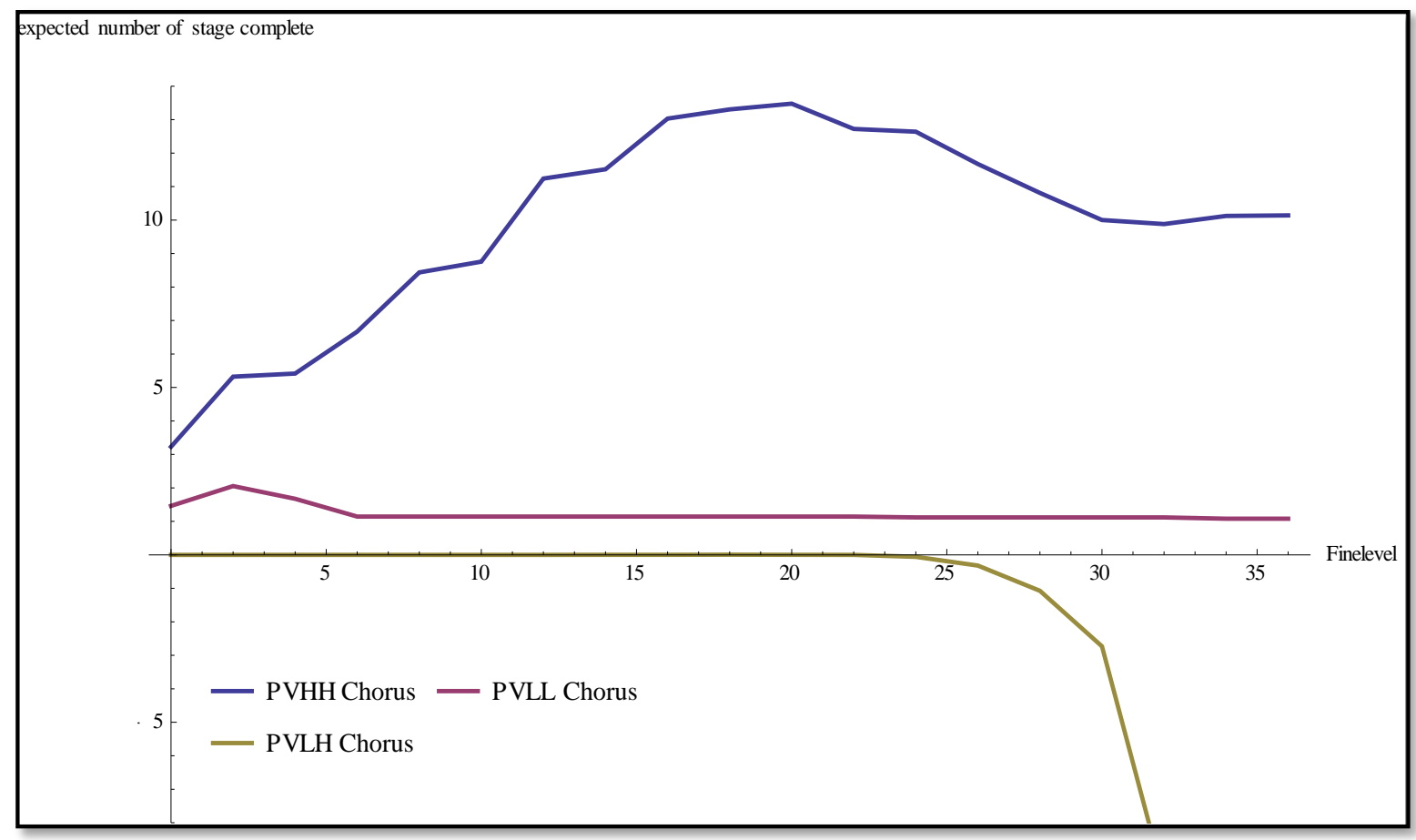

For the simple fine scheme, we rationalize the minimum level of conflict by considering the present values to CFH of individual cities (under Chorus' optimal policy). Unsurprisingly, we observe that PV LL and PV LH under the simple fine scheme (at graph 7.20) follow the

\footnotetext{
${ }^{108}$ Refer to our discussion about the optimal proportional subsidy at section 7.3.1.

${ }^{109}$ This is the case, especially for constructing city LH.
} 
same movements as those under the subsidy schemes ${ }^{110}$, and that they are dominated by the $\mathrm{PV} \mathrm{HH}$ as long as the level of fine is not extreme. Therefore, the minimum conflict level should be determined by PV HH, which has a peak when the level of the fine equals 20 . As a result, the conflict level reaches its minimum when the level of fine equals 20.

We plot the timing conflict and sequencing conflict measures at graph 7.21 and graph 7.22 respectively. As we can see, both timing and sequencing conflict measures are quite similar to those under the independent lump sum subsidy scheme. ${ }^{111}$ Of course, the corresponding turning point of the sequencing conflict measure and the intersections of the timing conflict measure are slightly different from those under the independent lump sum subsidy.

Graph 7.21: The timing conflict measures vary with the simple fine (target date at date 24)

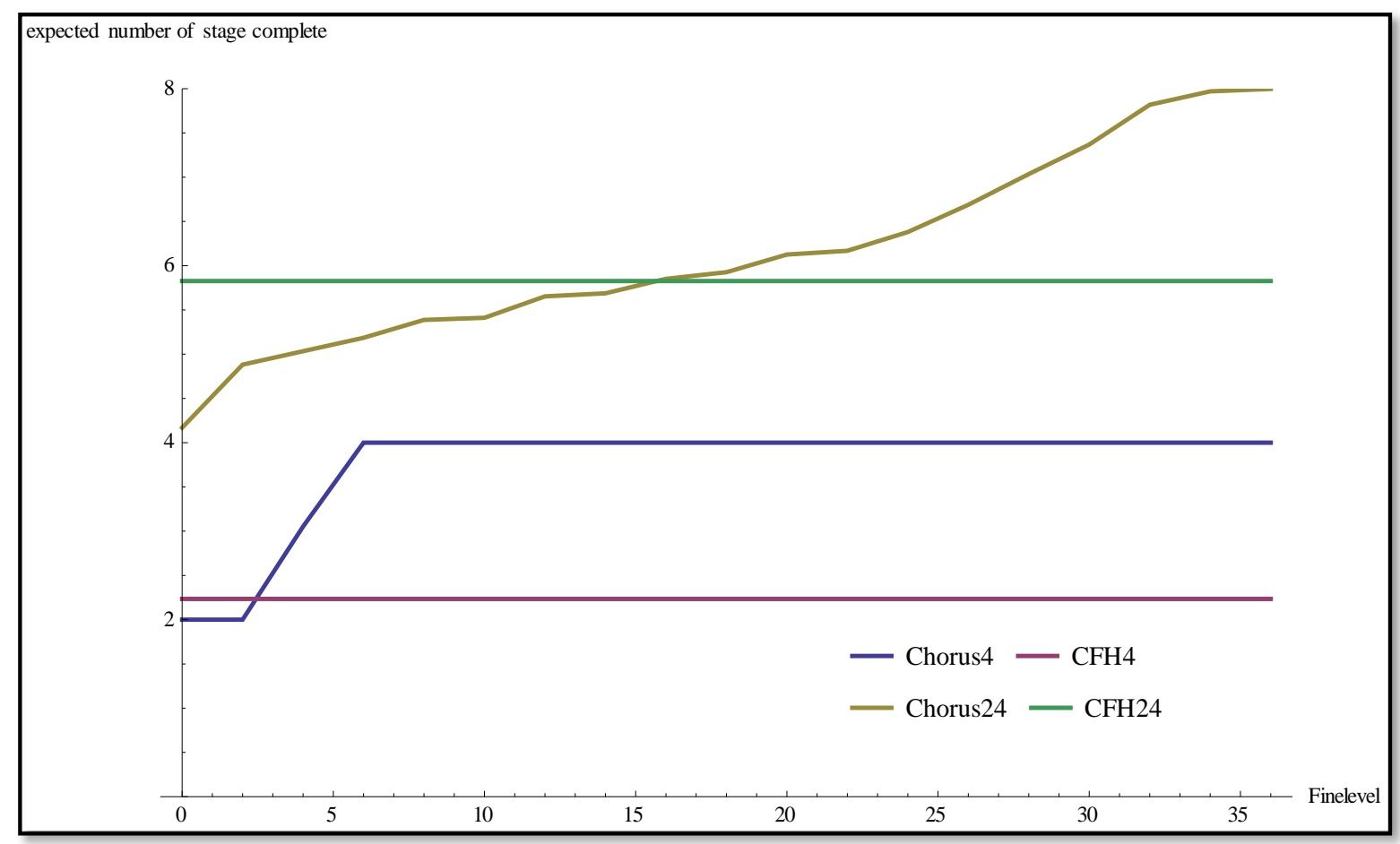

${ }^{110}$ This conclusion is drawn from a comparison of graph 7.2, graph 7.6, graph 7.10 and graph 7.14.

${ }^{111}$ This conclusion is drawn from a comparison of graph 7.11, graph 7.12, graph 7.21 and graph 7.22. 
Graph 7.22: The sequencing conflict measures vary with the simple fine (target date at date 24)

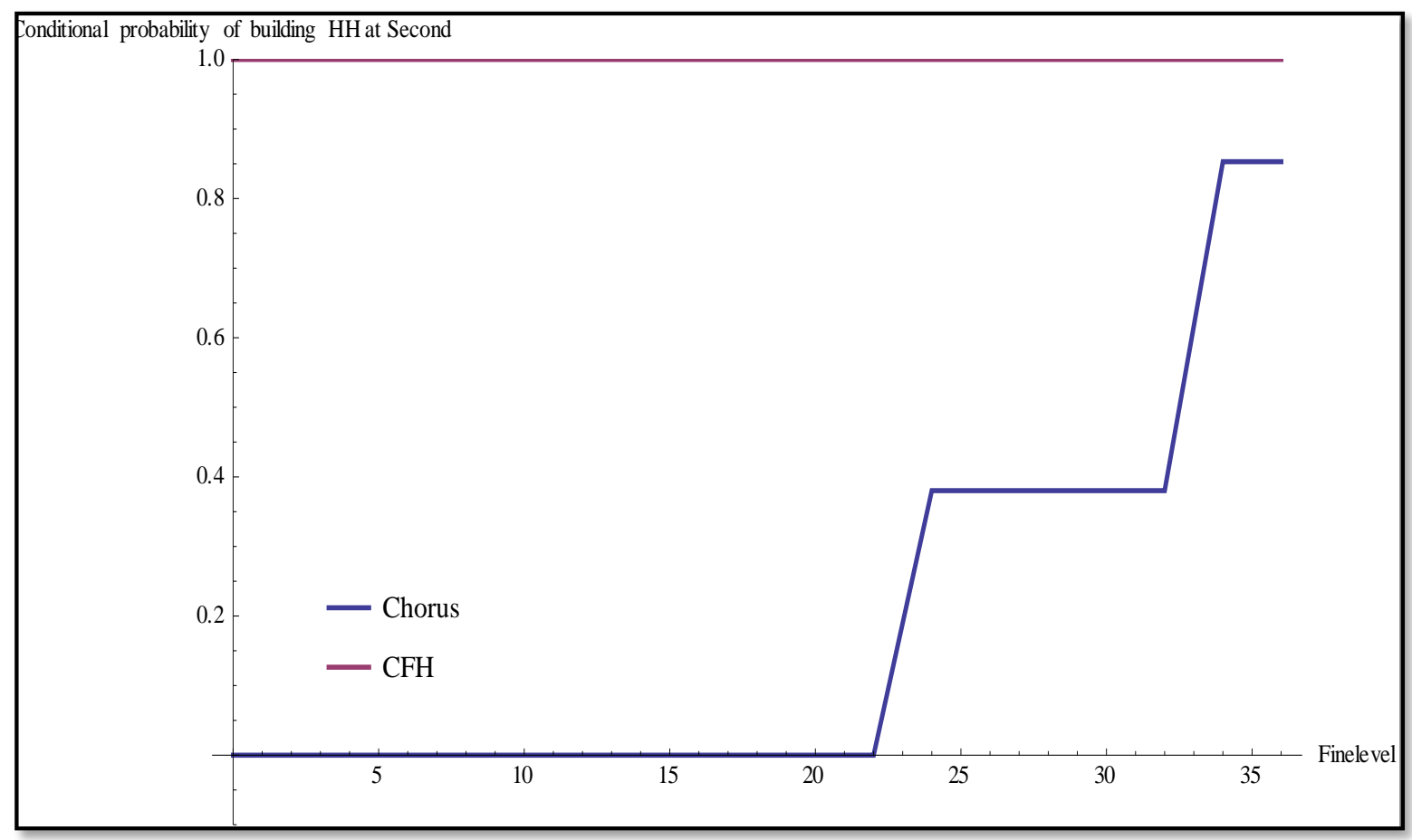

Graph 7.23: The present value of the UFB project for Chorus varies with the simple fine (target date at date 24)

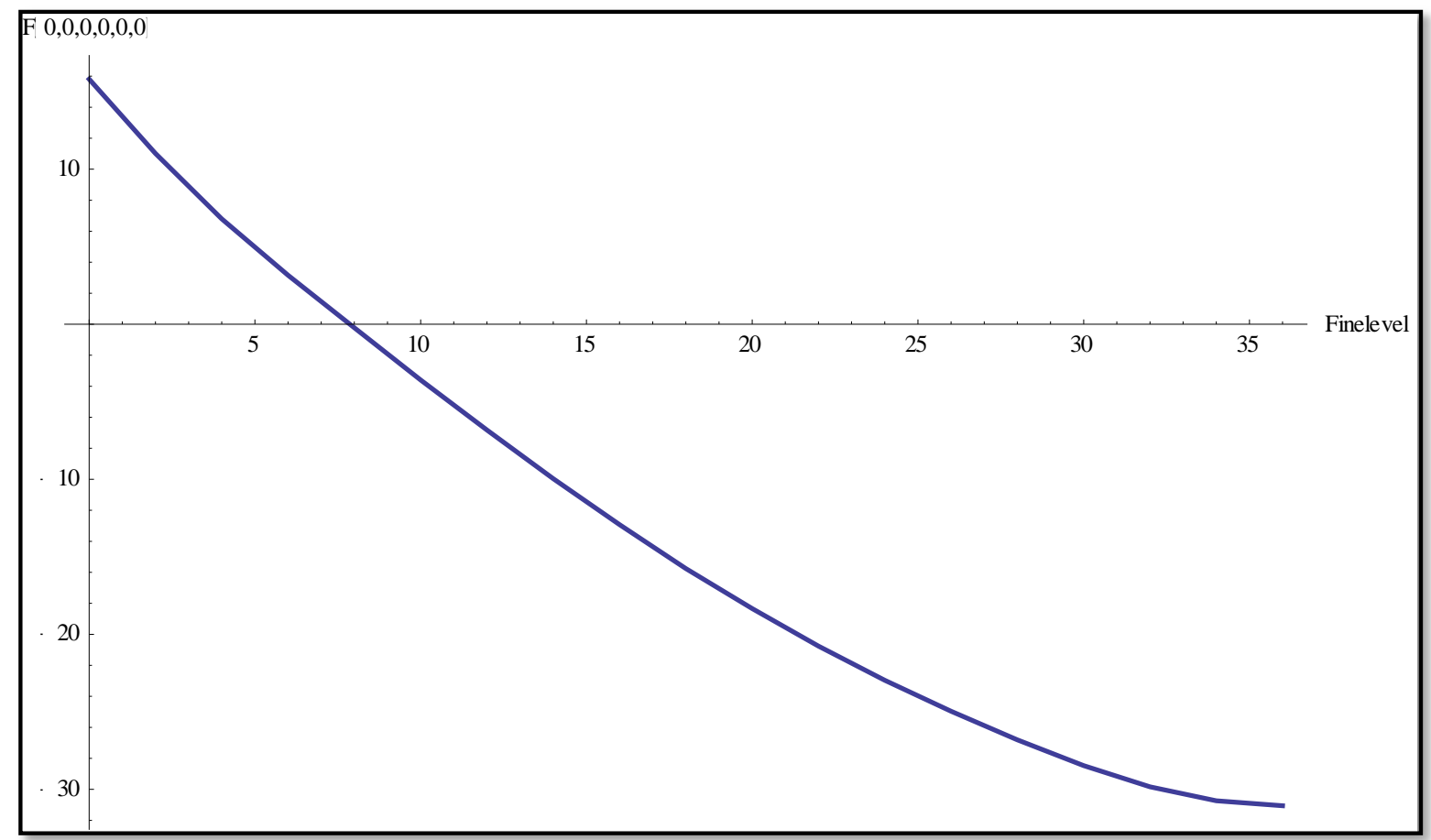


Although the simple fine scheme can largely eliminate the potential conflicts, in practice, there exists a participation constraint to Chorus when the fine scheme is undertaken. That is, Chorus does not want to be part of UFB network's construction after the fine reaches a certain level. Therefore, in order to investigate the participation constraint, we plot the present value of the UFB project for Chorus as a function of the fine level for the simple fine scheme at graph 7.23. As we can see, the present value of the UFB project for Chorus $(F[0,0,0,0,0,0])$ decreases with the level of fine and becomes negative when the level of fine goes over 8 , which implies that the feasible level of the simple fine cannot go beyond 8 .

From Graph 7.19, the minimum level of conflict that guarantees the participation of Chorus equals $6,{ }^{112}$ which implies the simple fine scheme is much less efficient than the subsidy schemes in reducing the conflict level.

\subsubsection{The two dimensional fine scheme}

In this section, we are going to extend our analysis of fines from a simple fine scheme to a two dimensional fine scheme. In practice, we construct the two dimensional fine scheme by adding a target at date 12 to the simple fine scheme. More specifically, this new target requires Chorus to finish at least two cities at date 12 . Otherwise, at date 12 , Chorus needs to pay the amounts Finelevel12 for only finishing one city and $2 \times$ Finelevel 12 for finishing no city. Similar to the simple fine scheme, Chorus is required to pay Finelevel24 at date 24 for each unfinished city. Accordingly, we have a 3D plot for how the conflict level varies with the two dimensional fine scheme at graph 7.24.

At graph 7.24, we find that the conflict level is dominated by the fine at date 24 (we label it as Finelevel24). That is, the increase of fine at date 24 dramatically decreases the conflict level, while the increase of fine at date 12 hardly changes the conflict level. ${ }^{113}$

\footnotetext{
${ }^{112}$ In practice, Chorus may not join the UFB project unless the present value of UFB project is beyond a certain positive value. Therefore, the minimum level of conflict that guarantees the participation of Chorus will shift up.

${ }^{113}$ The increase of fine at date 12 only slightly raises the level of conflict when the fine at date 24 is lower than 5.
} 


\section{Graph 7.24: The conflict level varies with the two dimensions fine}

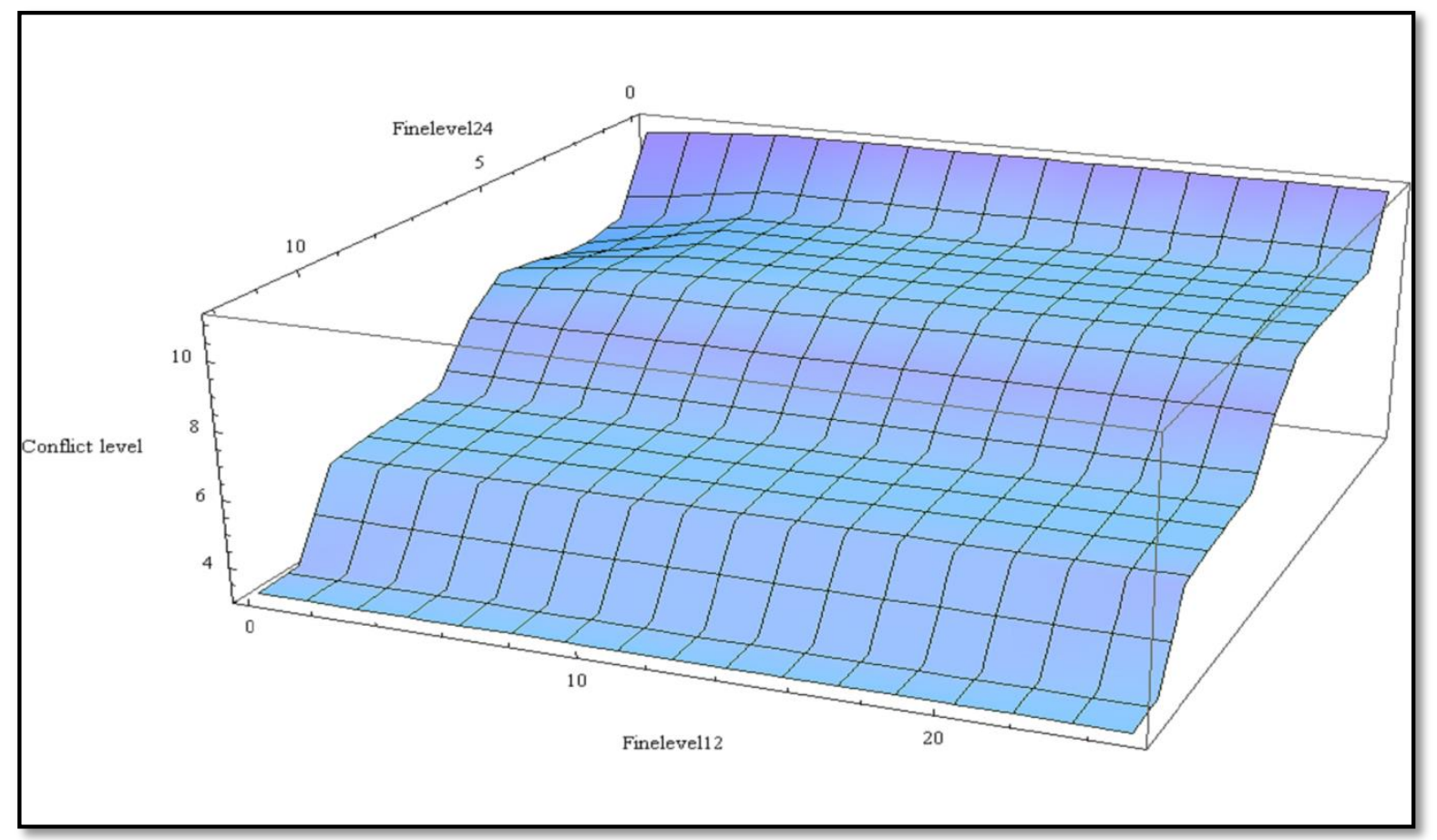

Graph 7.25 shows how the present values to CFH of the individual cities (under Chorus' optimal policy) vary with the two dimensional fine. Although city HL has the highest present value, it is hardly affected by any fines. (The present value to CFH of city HL is a smooth plane at graph 7.25.) However, the present value of city HH decreases with the fine at date 24. In contrast, the present value of city LL is barely affected by either fine most of the time. Nevertheless, the PV LL has a higher value when both fines are quite small. ${ }^{114}$ Recall that the difference between two parties in constructing city $\mathrm{HH}$ is the main driver of conflict. Therefore, the fine that determines the present value of city $\mathrm{HH}$, which is the fine at date 24 , should be the main determinant of the conflict level. Besides, we believe the conflict under the two-dimensional fine scheme is solely attributed to the timing conflict rather than the sequencing conflict. ${ }^{115}$

\footnotetext{
${ }^{114}$ The PV LL has a higher value when both fines at date 24 and date 12 are below 5 , which explains why the conflict level (at graph 7.24) sinks at this area.

${ }^{115}$ Since the conditional probability that Chorus wants to complete city $\mathrm{HH}$ second providing that at least two cities will be finished always equals 0 , the sequencing conflict measure under the two dimensional fine scheme is not affected by any fines.
} 


\section{Graph 7.25: The present values to CFH of the individual cities (under Chorus' optimal policy) vary with the two dimensional fine}

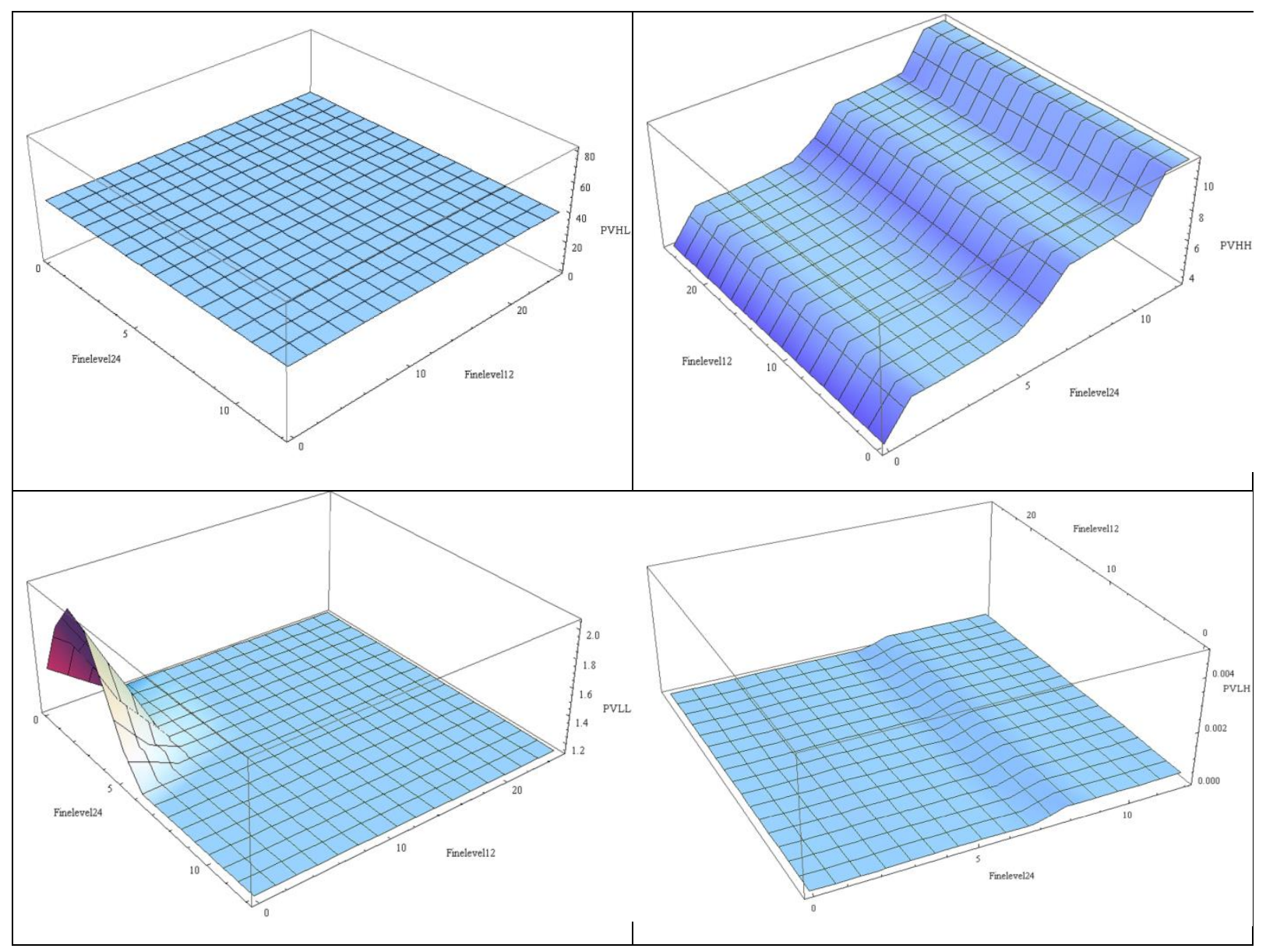

As for the simple fine scheme, we need to include the participation constraint to determine the feasible minimum conflict level for the two dimensional fine scheme. We plot the present value of the UFB project for Chorus as a function of the two fines at graph 7.26. This result is the same as that of the simple fine scheme. That is, the present value of the UFB project for Chorus $(F[0,0,0,0,0,0])$ decreases with the fine at date 24 and becomes negative when Finelevel24 goes over 8. Correspondingly, the minimum level of conflict (under the two dimensional fine scheme) that fits Chorus's participation constraint is equal to $6,{ }^{116}$ which suggests the two dimensional fine scheme does not perform much more efficiently than the simple fine scheme in reducing the conflict level.

${ }^{116}$ Similarly, the minimum conflict may shift up when the participation constraint not longer equals 0. 


\section{Graph 7.26: The present value of the UFB project for Chorus varies with the two}

\section{dimensional fine (target date at date 24)}

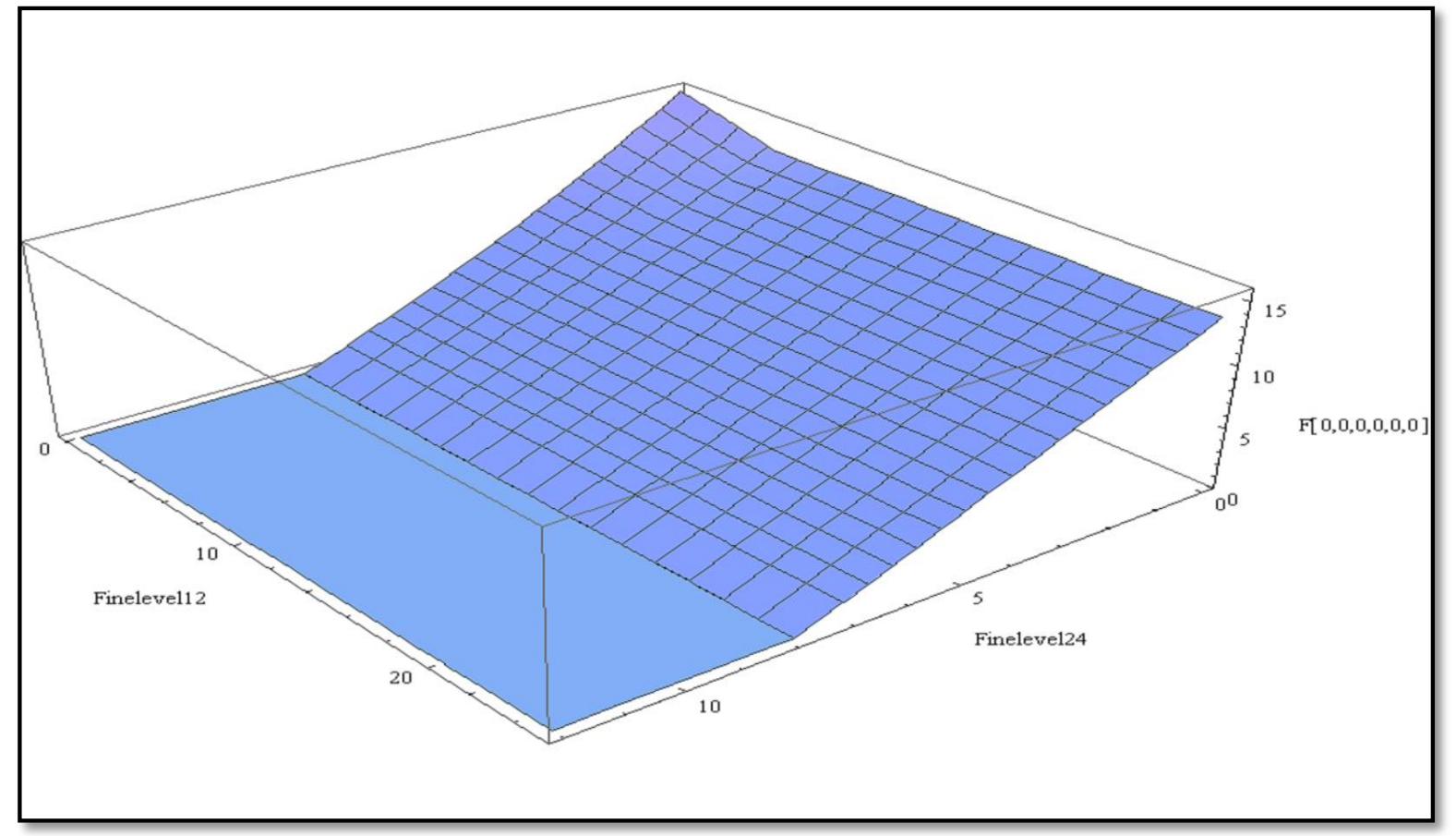

\subsubsection{Comments on the fine schemes}

In summary, if Chorus could be forced to take part (so that the participation constraint was not present) the fine schemes seem to work quite well in managing the conflict. ${ }^{117}$ However, the involvement of the participation constraint restricts the level of fine $\mathrm{CFH}$ may choose, which results in the fine schemes being much less efficient than the subsidy schemes in reducing the conflict level. Besides, we find that a shift from the simple fine scheme to the two dimensional fine scheme makes no improvement in conflict reduction.

\footnotetext{
${ }^{117}$ Even the simple fine scheme has a better performance than the fixed and independent lump sum subsidy in reducing the conflict level.
} 


\section{Chapter 8: Concluding remarks}

This thesis develops a model that investigates aspects of New Zealand's largest PPP project. The model features four cities with different demand and construction-cost characteristics. It is used to study the different choices of Chorus and CFH. Using a real options approach, we identify two sorts of potential conflicts between the two parties: a timing conflict about the number of cities the two parties would like to develop in each period and a sequencing conflict about the order in which the UFB network is rolled out in different cities. Inspired by the incomplete contracting and information asymmetry literatures, we introduce several incentive schemes (including four subsidy schemes and two fine schemes) that help manage the possible conflicts. By comparing their ability to reduce the potential conflicts and sensitivity analysis, we end up with four main findings in this study.

First, the magnitude of the conflict is a non-monotonic function of the inter-city demand differences $(\boldsymbol{D R})$ and the inter-city construction-cost differences $(\boldsymbol{C R})$; it is an increasing function of the ratio of consumer surplus to the square of producer surplus (Con) and of demand volatility. Since Con and demand volatility are not within the public party's control anyway, CFH may minimize the conflicts through "choosing” either $\boldsymbol{D R}$ or $\boldsymbol{C R}$. These parameters are treated as exogenous in our analysis. However, in practice, $\mathrm{CFH}$ could influence the values of $\boldsymbol{D R}$ and $\boldsymbol{C R}$ by the way it divides the country. That is, in order to make both parties agree on the construction order and construction timing, either CFH would like to make the cities as heterogeneous as possible (in this way, CFH wants to make either $\boldsymbol{D R}$ or $\boldsymbol{C R}$ as big as possible when the other parameters are fixed); or it would like to make the cities as homogeneous as possible (in this way, CFH wants to make either $\boldsymbol{D R}$ or $\boldsymbol{C R}$ close to 1 when the other parameters are fixed). However, the first method may not be a good solution for $\mathrm{CFH}$. This is because when $\mathrm{CFH}$ arranges cities in groups that are as heterogeneous as possible, it not only reduces the conflicts between the two parties but also increases the likelihood that Chorus may abandon part of project (this is not CFH's intention). For example, if CFH groups all the high demand cities to one city like Auckland and all the low demand cities to one city like Hamilton, ${ }^{118}$ Chorus may abandon Hamilton quite easily if the demand turns out to be low. Nevertheless, if Chorus breaks up the whole country more equally to one city like Wellington and the other city like Christchurch, ${ }^{119}$

\footnotetext{
${ }^{118}$ Auckland is the city with largest population density, while Hamilton is the city with smallest population density. This implies the UFB roll-out in Auckland may be much more profitable than that in Hamilton. ${ }^{119}$ Wellington and Christchurch shares the similar population density.
} 
Chorus may abandon neither city unless the demand is extremely low. Beside, by dividing the whole country into a very big city and a very small city (this is one of the simplest way to make the cities as heterogeneous as possible), $\mathrm{CFH}$ hands over almost all decision flexibility to Chorus, which may not be optimal for $\mathrm{CFH} .{ }^{120}$ Therefore, in practice, $\mathrm{CFH}$ should break up the country into homogeneous regions as much as possible when it designs the UFB deployment plan.

Second, the demand relevant lump sum subsidy has the best performance among all included incentive schemes in controlling the possible conflicts. More specifically, the demand relevant lump sum subsidy scheme works better than the proportional subsidy scheme ${ }^{121}$ (a cost dependent subsidy scheme) in reducing the potential conflicts but comes with the lower monitoring costs. The above finding suggests $\mathrm{CFH}$ should design the incentive scheme using information based on demand rather than information based on construction expenditures. This is consistent with what $\mathrm{CFH}$ is doing in the real world. Recall that $\mathrm{CFH}$ has claimed to subsidize Chorus a fixed amount of money for each household Chorus is going to pass on the UFB roll-out, which implies that the subsidy is insensitive to the construction expenditures per-household. On the other hand, Chorus is required to pay back the subsidy at an earlier date if the fibre uptake rate is below $20 \%$ in 2019. In other words, the subsidy Chorus receives has a higher present value when the demand turns out to high, which indicates the incentive scheme is sensitive to the demand.

Third, the conflict level becomes quite sensitive to the subsidy scheme in two cases. A) When either $\boldsymbol{D R}$ or $\boldsymbol{C R}$ turns out to be modest; B) When either $\boldsymbol{C o n}$ or $\boldsymbol{\sigma}$ turns out to be large. The above result may provide some suggestions in managing the optimal subsidy. That is, CFH should avoid setting the subsidy rate either too high or too low when either $\boldsymbol{D R}$ or $\boldsymbol{C R}$ turns out to be modest. Otherwise, the "wrong" subsidy rate set by CFH may result in a large social welfare loss due to the high conflict level. However, when $\boldsymbol{D R}$ is extreme (either too big or too small), CFH only needs to avoid setting the subsidy rate too high otherwise Chorus is encouraged to build up city LH. In contrast, when either Con or $\sigma$

\footnotetext{
${ }^{120}$ In the incomplete contract view, dividing the whole UFB project into a very big city and a very small city is the same as treating the whole UFB project as a sole city (since the small city does not matter), which makes the contract as incomplete as possible. This may eliminate the conflict completely, but at the same time, it limits CFH's ability to specify its construction preference through a formal contract. For example, CFH is no longer able to specify the construction order between cities (such as which city goes first and which city goes next) and constructing timing (that is the numbers of city that CFH want Chorus to finish). Therefore, Chorus may be able to roll out the UFB project in a way it likes, which may not be optimal to CFH.

${ }^{121}$ The proportional subsidy scheme has the second best performance in reducing the conflict.
} 
becomes relatively large, $\mathrm{CFH}$ should provide a high level subsidy to Chorus. Actually, a high level subsidy works regardless of $\boldsymbol{C o n}$. In reality, these model parameters $(\boldsymbol{D R}, \boldsymbol{C R}$, Con and $\sigma$ ) may not be constant for CFH over time. ${ }^{122} \mathrm{CFH}$ 's estimation of these model parameters may sometimes serve as the warning signs in managing the subsidy scheme. For example, when either $\boldsymbol{D R}$ or $\boldsymbol{C} \boldsymbol{R}$ is close to certain modest numbers, a relatively small change of either parameter may lead the conflict level from the area where the conflict level is insensitive to the subsidy rate to the area where the conflict level is very sensitive to the subsidy rate. This indicates that $\mathrm{CFH}$ may need to manage the subsidy rate very carefully in case a small change of $\boldsymbol{D R}$ or $\boldsymbol{C R}$ dramatically raises the conflict level. In contrast, when either $\boldsymbol{D} \boldsymbol{R}$ or $\boldsymbol{C} \boldsymbol{R}$ is an extreme number, CFH may not need to worry about the subsidy rate since the conflict level is quite low regardless of the subsidy rate, and more important, it takes a relatively big change of $\boldsymbol{D R}$ or $\boldsymbol{C R}$ (which means more unlikely to happen) to lead the conflict level from the area where the conflict level is insensitive to the subsidy rate to the area where the conflict level is very sensitive to the subsidy rate. Further on, CFH may need to be very careful in the parameter estimation. This is because if $\mathrm{CFH}$ get a wrong value for the parameter, it may provide Chorus a wrong level of subsidy. Therefore, the corresponding conflict between these two parties will be massive.

Last, although the fine schemes come with the low monitoring cost, they are generally outperformed by the subsidy schemes due to their participation constraints. Therefore, in the case where only one incentive scheme could be used, CFH should always go for a subsidy scheme rather than a fine scheme. This is consistent with the fact that $\mathrm{CFH}$ adopted a subsidy scheme as the main incentive scheme in UFB roll-out. A fine up to $\$ 350$ million only is introduced to discourage Chorus from abandoning the UFB project. However, if CFH would like to combine a fine scheme with a subsidy scheme (which helps relaxing the participation constraint), the ideal candidate for the add-on subsidy scheme will be the independent lump sum subsidy scheme. ${ }^{123}$ We may need to realise that it is very difficult to

\footnotetext{
${ }^{122}$ Even when these model parameters are constant over time, CFH may not know their true values when CFH designs the incentive scheme and signs up the UFB contract with Chorus. In this case, CFH can only have estimation on these model parameters based on the information it has collected. As we know, the estimated parameters may not necessarily be consistent with their true values. Therefore, in CFH's understanding, these parameters may not be constant. Besides, these parameters do experience some shocks under certain circumstances. One good example may be the 2011 Christchurch's earthquake. The population of Christchurch has dropped 2.4 per cent after the city experienced a magnitude 6.3 earthquake, which may potentially increase the Cost Ratio but reduces the demand ratio due to the reduction of population density. ${ }^{123}$ In the cost-benefit view, the fixed subsidy scheme is strictly dominated by the independent lump sum subsidy scheme, while the proportional subsidy scheme is strictly dominated by the demand relevant lump sum subsidy scheme. Therefore, the fixed subsidy and proportional subsidy scheme should be excluded
} 
find out the optimal combination between a fine scheme and a subsidy scheme. This is because the fine scheme has so many choice variables.

In this thesis, we have provided several suggestions to $\mathrm{CFH}$ that may help manage the conflict when the UFB rollout is conducted in a form of private public partnership. Through the lens of incomplete contracting, the more $\mathrm{CFH}$ can specify what it wants in designing the UFB deployment plan and incentive scheme, the less freedom CFH may pass to Chorus. Therefore the less conflict may rise on the UFB rollout. However, the completeness of contracting does not come for free and more extra monitoring costs may be involved when CFH are making the UFB contract more complete. Therefore, there is always a tradeoff between the contract completeness and monitoring cost when $\mathrm{CFH}$ is contracting with Chorus. Overall, this thesis provides a better understanding about the above tradeoff under a PPP project, and more important, several practical suggestions have been derived to improve the optimal contracting.

anyway. However, the demand relevant lump sum subsidy scheme is never an ideal add-on to the fine schemes. This is because, if CFH is looking for the minimum conflict, it should always employ the demand relevant lump sum subsidy alone since the demand relevant lump sum subsidy scheme can eliminate the conflict almost. On the other hand, if $\mathrm{CFH}$ is looking for the minimum monitoring cost, the independent lump sum subsidy should be preferred since it comes with the lowest monitoring cost. 


\section{References}

Alonso-Conde, A., Brown, C. AND Rojo-Suarez, J., 2007, Public private partnerships: incentives, risk transfer and real options, The Review of Financial Economics, 16, 335-349.

Amram, M. AND Kulatilaka, N., 1999, Real Options: Managing Strategic Investment in an Uncertain World, Harvard Business School Press.

Bennett, J. AND lossa, E., 2006, Building and Managing Facilities for Public Services, The Journal of Public Economics, 90, 2143-2160.

Black, F. AND Scholes, M.S., 1973, The pricing of options and corporate liabilities, Journal of Political Economy, 81, 637-59.

Chen, H.Y. AND Qin, R.W., 2012, Real options as an incentive scheme for managing revenues in transportation infrastructure projects, International Journal of Revenue Management, 6, 77-101.

Copeland, T.E. AND Antikarov, V., 2001, Real Options: A Practitioner's Guide, Texere LLC.

Cox, J. C., S. A. Ross, AND M. Rubinstein., 1979, Option Pricing: A Simplified Approach, Journal of Financial Economics, 7, 229-64.

Crown Fibre Holdings Limited annual report, 2012, http://www.crownfibre.govt.nz/wpcontent/uploads/2011/10/CFH-Annual-Report-2013.pdf

Dixit, A.K. AND Pindyck, R.S., 1994, Investment under Uncertainty, Princeton University Press. 
Engle, E., Fischer, R. AND Galetovic, A., 2013, The basic public finance of Public-Private Partnerships, Journal of the European Association, 4, 83-111.

Ford, D.N., Lander, D.M. AND Voyer, J.J., 2002, A real options approach to valuing strategic flexibility in uncertain construction projects, Construction Management and Economics, 20, 343-351.

Garvin, M.J. AND Cheah, C. Y. J., 2004, Valuation techniques for infrastructure investment decisions, Construction Management and Economics, 22, 373-383.

Grimes, A., 2011, Building Bridges: Treating a New Transport Link as a Real Option, Motu Working Paper, 11-12.

Grimsey, D. AND Lewis, M., 2007, Public Private Partnerships and Public Procurement, Agenda: A Journal of Policy Analysis and Reform, 14, 171-188.

Guthrie, G., 2009, Real Options in Theory and Practice, Oxford University Press.

Hart, O., Shleifer, A. AND Vishny, R., 1997, The proper scope of government: theory and applications to prisons, The Quarterly Journal of Economics, 112, 1119-1158.

Hart, O., 2003, Incomplete contracts and public ownership: remarks and an application to public-private partnership, The Economic Journal, 113, 69-76.

HM Treasury, 2003a, The Green Book - Appraisal and Evaluation in Central Government, TSO, London.

HM Treasury, 2003b, PFI: Meeting the Investment Challenge, HMSO, Norwich. 
Katz, D., 2006, Financing Infrastructure Projects: Public Private Partnerships (PPPs), New Zealand Treasury Policy Perspectives Paper 06/02.

Laffont, J.J. AND J. Tirole, 1993, A theory of incentives in regulation and procurement, MIT Press.

Liu, J. AND Cheah, C. Y. J., 2009, Real option application in PPP/PFI project negotiation, Construction Management and Economics, 27, 331-342.

Liu, J., Yu, X.B. AND Cheah, C. Y. J., 2014, Evaluation of restrictive competition in PPP projects using real option approach, International Journal of Project Management, 32, 473481.

Martimort, D. AND Pouyet, J., 2008, Build It Not: Normative and Positive Theories of PublicPrivate Partnerships, The International Journal of Industrial Organization, 26, 393-411.

Merton, R.C., 1973, Theory of rational option pricing, Bell Journal of Economics and Management Science, 4, 141-83.

Milner, M., 2009, Fibre-to-the-Premise Cost Study, Milner Consulting Limited.

Mott MacDonald, 2002, Review of Large Public Procurement in the UK, HM Treasury, London.

National Audit Office, 2003, PFI: Construction Performance, Report by the Comptroller and Auditor General, HMSO, London.

National Audit Office, 2005, PFI: Construction Performance, Report by the Comptroller and Auditor General, HMSO, London. 
Network Infrastructure Project Agreement, 2011, http://www.crownfibre.govt.nz/crownpartners/agreements-with-ufb-partners/network-infrastructure-project-agreement-nipa24-may-2011/

Rocha Armada, M. J., Pereira, P. J. AND Rodrigues, A., 2012, Optimal subsidies and guarantees in public-private partnerships, The European Journal of Finance, 18, 469-495.

Rose S., 1998, Valuation of interacting real options in a toll road infrastructure project, The Quarterly Review of Economics and Finance, 38, Special Issue, 711-723.

Shan, L., Garvin, M.J. AND Kumar, R., 2010, Collar options to manage revenue risks in real toll public, private partnership transportation projects, Construction Management and Economics, 28, 1057-1069.

Submission in response to the Commerce Commission's Draft Determination to amend the price payable for the regulated service Chorus' unbundled bitstream access made under $\mathrm{s}$ 30R of the Telecommunications Act 2001, 2013, http://www.chorus.co.nz/file/14919/Chorus-UBA-Submission.pdf

Trigeorgis, L., 1993, Real Options and Interactions with Financial Flexibility, Financial Management, 22, 202-224.

Trigeorgis, L., 1996, Real Options: Managerial Flexibility and Strategy in Resource Allocation, The MIT Press.

Van Ham, H. AND Koppenjan, J., 2001, Building Public - Private Partnerships: Assessing and Managing Risks in Port Development, Public Management Review, 4, 593-616. 